\title{
Theoretical Description of Hydrogen Atom Scattering off Noble Metals
}

\author{
Dissertation \\ zur Erlangung des mathematisch-naturwissenschaftlichen Doktorgrades \\ "Doctor rerum naturalium" \\ der Georg-August-Universität Göttingen \\ im Promotionsprogramm Chemie \\ der Georg-August University School of Science (GAUSS)
}

vorgelegt von

Svenja Maria Janke

aus Flensburg

Göttingen, 2016 
Betreuungsausschuss

Prof. Dr. Alec M. Wodtke, Dynamics at Surfaces, Institute for Physical Chemistry, Max-Planck Institute for Biophysical Chemistry

Prof. Dr. Geert-Jan Kroes, Theoretical Chemistry, Leiden Institute of Chemistry (Netherlands)

Mitgliefer der Prüfungskommission

Referent: Prof. Dr. Alec M. Wodtke, Dynamics at Surfaces, Institute for Physical Chemistry, Max-Planck Institute for Biophysical Chemistry

Korefferent: Prof. Dr. Geert-Jan Kroes, Theoretical Chemistry, Leiden Institute of Chemistry (Netherlands)

Weitere Mitglieder der Prüfungskommission:

Prof. Dr. Ricardo Mata, Computational Chemistry and Biochemistry, Institute for Physical Chemistry

Dr. Tim Schäfer, Dynamics at Surfaces, Institute for Physical Chemistry

Prof. Dr. Jörg Schroeder, Institute for Physical Chemistry

Prof. Dr. Dirk Schwarzer, Reaction Dynamics, Max-Planck Institute for Biophysical Chemistry

Tag der mündlichen Prüfung: 13.05.2016 

"Omnia mea mecum porto."

Marcus Tullius Cicero

Thus far I have accomplished the Object of my Voyage, and that too in full time to return advantageously agreeable to my Orders. Should I be so unfortunate as to lose many of the Breadfruit Plants, it may be said, why did I not act against a loss which I might in some degree expect by not taking other Fruit. In answer to this I refer to my account on the 4th February where I have shown at a moderate estimation that I got laid in 389 additional Breadfruit Plants more than was supposed the ship would stow when I left England, and as to the others, considering them equally essential to the good of our West Indies it laid particularly with me how far I could be of service in carrying other Valuable Plants.

Wm Bligh

Tahiti, Wednesday, 01.04.1789 Extract from Bounty Logbook [1] 


\section{Abstract}

I have constructed a full-dimensional potential energy surface (PES) for a $\mathrm{H}$ atom interacting with a $\mathrm{Au}(111)$ surface by fitting the analytic form of the Effective Medium Theory [2] to density functional theory (DFT) energies. The fit used energies of the H-Au system with the Au atoms at their relaxed lattice positions as well as configurations with the Au atoms displaced from these positions. The procedure provides an accurate treatment for displacements of $\mathrm{Au}$ atoms and compares well to DFT energies for scattering of $\mathrm{H}$ atoms obtained from $a b$ initio molecular dynamics (AIMD). In adiabatic molecular dynamics simulation, the PES is capable of reproducing the energy loss behavior of AIMD. The effective medium theory also provides the background electron density which makes it possible to treat the excitation of energetically low lying electron hole pairs self-consistently in the frame of local density friction approximation. I have tested various incidence conditions for $\mathrm{H}$ scattering from $\mathrm{Au}(111)$ and find that in all cases, the energy loss by electron hole pair excitation is by far the most efficient pathway and dominates the scattering behavior and influences the adsorption mechanism significantly. Comparison to experiment confirms that energy loss to electron hole pairs is the dominant energy dissipation pathway. Molecular dynamics simulations including nonadiabatic effects for multiple incidence conditions are in very good agreement with experiment. Furthermore, the theory offers an explanation for the apparent lack of an isotope effect observed experimentally. I consider the effect of changes in the electron density and the inclusion of surface reconstruction and find the results are not much altered. For molecular dynamics simulations and fitting, I used a self-written program package to which I contributed substantially. 


\section{Acknowledgments}

The last four years that I spent working on the present project I experienced a wonderful time. Foremost of all, I would like to thank Prof. Dr. Alec Wodtke for providing me with the opportunity to work on an exceedingly interesting and rewarding project, for his excellent supervision and furtherance of my development as a scientist throughout the course of the work. I am truly grateful for this.

I would like to thank Dr. Alexander Kandratsenka for mentoring me in a wonderful manner, for all the patient explanations and for always being open to questions as well as very good company. My thanks belong to Prof. Dr. Daniel J. Auerbach for teaching me much about science, that there will always be new and exciting things to learn, and for all the good advice and help he has provided. Prof. Dr. Geert-Jan Kroes I would like to thank for the scientific support and his inspiring conversations.

My special thanks belong to all the people in the Wodtke research group and those associated with it for the warm and constructive atmosphere, for all their help, friendship and good company, especially for our often funny after-dinner conversations. - I shall miss you greatly.

To my family (especially my parents, my brother, my cousin Inga and my grandparents) I am ever so grateful for all the support and happiness they have given me and the faith they have in me. There is no better family than you.

My friends I would like to thank for all the good time we have spent together and for the loyal friendship they have shown, sometimes even with half the world between us.

In the course of my work on this thesis I was privileged to read the logbooks of the voyages of the HM Transport Bounty and the HMS Providence by William Bligh (later Vice Admiral of the Blue, F.R.S., R.N.). They taught me valuable lessons on humanity and science. For this, I am grateful. 


\section{Contents}

\begin{tabular}{ll}
\hline 1. Introduction & 1
\end{tabular}

2. Theoretical Methods $\quad 5$

2.1. The Born-Oppenheimer Approximation . . . . . . . . . . . . . . . . 5

2.2. Potential Energy Surfaces . . . . . . . . . . . . . . . . . . 8

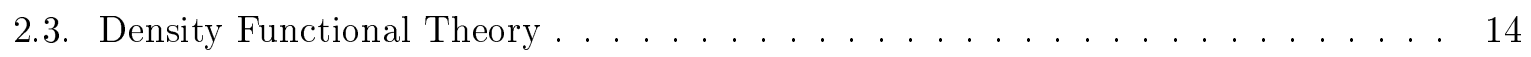

SRP-Functional . . . . . . . . . . . . . . . . . . . . 17

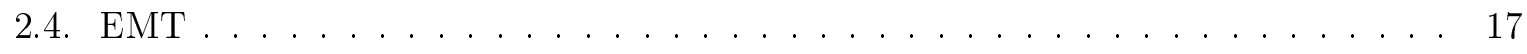

2.5. Local Density Friction Approximation . . . . . . . . . . . . . . . . . . 23

2.5.1. Recent Applications of LDFA . . . . . . . . . . . . . . . . . . . 24

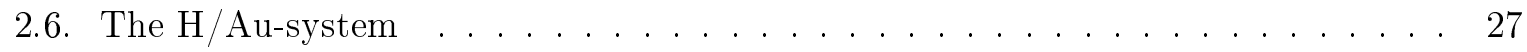

Bulk and Surface Properties . . . . . . . . . . . . . . . 27

2.6.1. Fcc-metals and Gold . . . . . . . . . . . . . . . . . . . . . . 28

Au surface structure . . . . . . . . . . . . . . . . . . . . 28

2.6.2. Hydrogen in Interaction with Metals . . . . . . . . . . . . . . . . 30

2.7. Fitting Procedure . . . . . . . . . . . . . . . . . . . . 32

2.7.1. The DFT $a b$ initio input data set . . . . . . . . . . . . . . . . 32

2.7.2. The Fit . . . . . . . . . . . . . . . . . . . . 34

2.8. MD simulations . . . . . . . . . . . . . . . . . . . . . . . 34

3. Results 39

3.1. DFT-Calculations. . . . . . . . . . . . . . . . . . . . 39

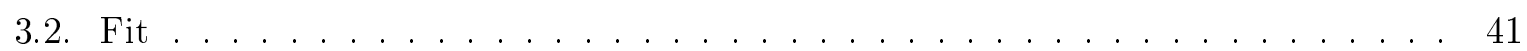

3.2.1. Comparison to earlier EMT parametrizations . . . . . . . . . . . . . 47

3.2.2. Comparison of different fits . . . . . . . . . . . . . . 50

3.3. MD Simulation of $\mathrm{H}$ scattering from $\mathrm{Au}(111)$ with Various Conditions . . . . . . 56

3.3.1. Detailed analysis of scattering outcomes . . . . . . . . . . . 56

Single-Bounce Events . . . . . . . . . . . . . . . . . . 61

Double-bounces Events . . . . . . . . . . . . . . . . . . 65

Scattering after Penetration of the Surface Layer . . . . . . . . . . . 71 
Further investigations . . . . . . . . . . . . . . 72

Angular Distribution . . . . . . . . . . . . . . . . 74

Summary . . . . . . . . . . . . . . . . . . . . . 75

3.3.2. The Influence of Temperature on the Energy Loss Distribution . . . . . . 79

3.3.3. The Influence of Incidence Energy ～. . . . . . . . . . . . . . . . . . . 90

3.3.4. The Dependence of Scattering on the Incidence Angles . . . . . . . . . . . 99

3.3.5. The Influence of Surface Structure on Scattering . . . . . . . . . . . 107

3.3.6. Isotope effect . . . . . . . . . . . . . . . . . . . . . . 110

3.3.7. The Influence of Changes in Electron Density . . . . . . . . . . . . . . . 114

3.3.8. Conclusion . . . . . . . . . . . . . . . . . . . . . . . . 119

3.4. Surface Reconstruction . . . . . . . . . . . . . . . . . . . . . . . . 121

4. Comparison of MD simulations to Experimental Results 125

4.1. Energy Loss Distributions . . . . . . . . . . . . . . . . . . . . . 125

4.2. Angular Distributions . . . . . . . . . . . . . . . . . . . . 131

4.3. Improvement of comparison . . . . . . . . . . . . . . . . . . 133

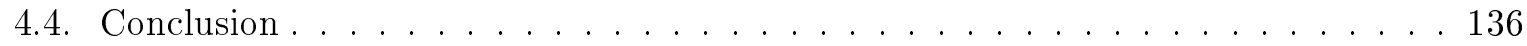

5. Conclusions and Outlook 139

\begin{tabular}{ll}
\hline A. Appendix & 157
\end{tabular}

A.1. VASP . . . . . . . . . . . . . . . . . . . . . 157

A.1.1. Input files . . . . . . . . . . . . . . . . . . 157

INCAR . . . . . . . . . . . . . . . . . . . . . . . . . 158

POSCAR . . . . . . . . . . . . . . . . . . 159

POTCAR . . . . . . . . . . . . . . . . . . . 160

KPOINTS . . . . . . . . . . . . . . . . . . . . . 160

A.1.2. Output Files . . . . . . . . . . . . . . . . . 160

A.1.3. SRP input files . . . . . . . . . . . . . . . . . . . 160

A.1.4. PBE input files . . . . . . . . . . . . . . . . . . 163

A.1.5. PW91 input files . . . . . . . . . . . . . . . . . . . 165

A.1.6. RPBE input files . . . . . . . . . . . . . . . . . 167

\begin{tabular}{lr}
\hline B. MD_Tian Program & 169
\end{tabular}

B.1. The EMT-parameter file . . . . . . . . . . . . . . . . . . . . . . . . . . . . 169

B.2. The Configuration files . . . . . . . . . . . . . . . . . . 170

B.3. The Control File for MD simulations . . . . . . . . . . . . . . . . . . 171

B.3.1. Output files . . . . . . . . . . . . . . . . . . 173 
B.4. The Propagation . . . . . . . . . . . . . . . . . . . . . . 174

B.4.1. Propagation Algorithms . . . . . . . . . . . . . . . . . . . 174

B.4.2. Fitting Procedure . . . . . . . . . . . . . . . . . . . 175

B.4.3. Surface Annealing . . . . . . . . . . . . . . . . . . 176

B.4.4. Influence of MD Simulation Conditions on Scattering Results . . . . . . 177

B.4.5. Disintegration Temperature . . . . . . . . . . . . . . . 178 



\section{List of Abbreviations}

ad adiabatic

3D-grid three dimensional grid

AIMD Ab Initio Molecular Dynamics

AIMDEF $\quad A b$ Initio Molecular Dynamics including Electronic Friction

AIMDEFp Ab Initio Molecular Dynamics including Electronic Friction in the post-facto approach

ASA Atomic Sphere Approximation

BOA Born-Oppenheimer Approximation

CRP Corrugation Reducing Procedure

DELD differential energy loss distribution

DFT Density Functional Theory

EAM Embedding Atom Method

ehp electron hole pairs

ELD energy loss distribution

EMT Effective Medium Theory

$f c c \quad$ face centered cubic

GGA Generalized Gradient Approximation

hcp hexagonal close-packed

LDA local density approximation

LDFA local density friction approximation

LEPS London-Eyring-Polanyi-Sato

MD Molecular dynamics

MDEF molecular dynamics with electronic friction

MDEF-SS molecular dynamics with electronic friction for a frozen/solid surface

MS Modified Shepard

na nonadiabatic

rms root mean square (error)

PAW projector augmented wave

PBE Perdew-Burke-Ernzerhof, a GGA-functional

Continued on next page 
PES Potential Energy Surface

PW91 Perdew-Wang '91, a GGA-functional

RMM-DIIS residual minimisation method with direct inversion of iterative subspace

RPBE revised Perdew-Burke-Ernzerhof, a GGA-functional

SRP Specific Reaction Parameter, a GGA-functional

TELD total energy loss distribution

TOF time-of-flight

TPD temperature programmed desorption

TUT tetrahedral under top

US-PP ultra soft pseudo-potentials

VASP Vienna ab initio Simulation Package

VMD Visual Molecular Dynamics

WS Wigner Seitz 


\title{
List of Physical Constants
}

\author{
$a_{0} \quad$ lattice constant \\ $B$ bulk modulus \\ $C_{44}$ shear modulus \\ $h \quad$ Planck constant \\ $\hbar \quad$ reduced Planck constant \\ $k_{\mathrm{B}} \quad$ Boltzmann constant \\ $R \quad$ ideal gas constant \\ $T$ temperature
}





\section{Introduction}

The present project's purpose was the construction of a full-dimensional potential energy surface (PES) to describe the interaction of a $\mathrm{H}$ atom in interaction with a $\mathrm{Au}(111)$ surface and to develop an understanding of the basic mechanisms of energy transfer and of the adsorption in $\mathrm{H}$ collisions with metal surfaces: in contrast to ab initio molecular dynamics (AIMD) simulations (e.g. [3, 4]) the PES allows simulations on a ps-timescale, while in contrast to other PESs (e.g. [5, 6]) all degrees of freedom, including those of the surface, are treated. The PES provides background electron densities, which makes the self-consistent treatment of energy loss to electron hole pairs (ehp) by means of local density friction approximation possible. All this allows me to make discoveries on the nature of scattering processes taking place on long time scales, adsorption mechanisms and energy loss mechanisms that would have otherwise been unfeasible.

To understand the importance of potential energy surfaces, it is illustrative to consider a comparison to geographical maps: potential energy surfaces could be considered as maps of an electronically adiabatic state that, instead of the geographic surrounding, yield positiondependent information on the energetic landscape. While the maps of old offered knowledge to gain save passage or find islands again if you were able to determine your position, potential energy surfaces offer understanding about chemical transformation. Indeed, many important discoveries have been made in the field of chemical reaction dynamics from the calculation of the motion of reactants and reagents on the PES. For gas phase reactions, this involves the discovery of the Polanyi [7] rules that describe the influence of the reactant's vibration and translation on the chemical reactivity based on the landscape of the PES, as well as roaming reactions with subsequent hydrogen abstraction [8] or the discovery that quantum mechanical resonances [9] can have an influence on the product's behavior after chemical reactions.

The process of map making has always been an arduous one; for potential energy surfaces, the problems are as manifold: if the use of a PES is extended from gas phase to reactions involving more complex systems such as particles in interaction with surfaces, a huge number of degrees of freedom needs to be taken into account for not only does the particle need description, but the motion of the substrate atoms cannot be ignored [10-12]. Furthermore, metals do not have a bandgap so that the adiabatic approximation might well fail [13, 14], making it necessary to account for the interaction between nuclear and electronic degrees of freedom during trajectories[15, 16]. Additionally, especially when small particles are involved, the influence of 
such quantum effects as e.g. tunneling [17] may need to be taken into account: The understanding of $\mathrm{H}$ atom adsorption or scattering on metal surfaces is perhaps the simplest chemical surface reaction. But just as charting a harbor with all its currents is difficult and challenging, developing an understanding of how the process proceeds [3, 4, 18, 20] and applying PESs to this condensed phase is a complex and challenging undertaking [21-23].

Adsorption of atomic hydrogen has first been studied by Langmuir [24] and is important for many fields, be it heterogeneous catalysis [25], interstellar hydrogen on dust grains [26], nuclear fusion in tokamaks or storage of $\mathrm{H}$ [27]. Still it is not self-evident how $\mathrm{H}$ impinging on the surface loses its initial energy and thus comes to adsorption. It may either proceed by conversion of motion normal to the surface motion into parallel momentum and the following energy loss to phonons due to interactions with the corrugated surface structure or subsurface penetration [21, 23]. But this energy transfer is increasingly more inefficient the heavier the surface atoms are relative to the mass of the impinging atoms. Thus, the $\mathrm{H}$ atom needs to undergo many collisions before it may stick to the surface, suggesting long interaction time on the order of ps. It may also involve energy loss to electron hole pair excitation, describing a failure of the Born-Oppenheimer Approximation (BOA). Already in 1979 Nørskov and Lundqvist [18] suggested that the excitation of ehp in a $\mathrm{H}$ atom-metal interaction is conceivable at higher speeds of the $\mathrm{H}$ atom and might be of considerable importance due to the inefficient energy loss to phonons. Because of the continuum of electronic states at a metal surface, the Born-Oppenheimer Approximation should already appear suspicious [15]. A very promising system to determine the importance and role of ehp excitation for $\mathrm{H}$ on metal is therefore $\mathrm{H}$ on $\mathrm{Au}$; the $\mathrm{Au}$ atoms are so heavy that purely adiabatic energy loss to them is very inefficient which should make it easier to detect energy loss to ehp.

To determine which of these two mechanisms is dominant, both of them need to be modeled theoretically. This modeling not only involves the treatment of a large number of degrees of freedom over a long time scale but also the inclusion of nonadiabatic effects, both of them in a selfconsistent manner. In this respect, ab initio molecular dynamics [28, 29] might open up a feasible route: it has already been used to describe interaction of $\mathrm{H}$ with $\mathrm{Au}(111)$ [3, 4] and $\mathrm{H}$ with $\mathrm{Pd}$ [19] where the electron densities and forces were calculated on-the-fly with density functional theory (DFT) on the level of the generalized gradient approximation (GGA). Furthermore, BlancoRey [19] et al. showed that it allows the calculation of low-lying ehp excitation by means of local density friction approximation on-the-fly (LDFA) [30, 31]. While this makes it possible to treat both effects on a trajectory, the computational cost of AIMD weighs heavy: for the calculation of already a minor number of trajectories $(\sim 1000)$, the interaction time of $\mathrm{H}$ with the surface is limited to time scales much below 1 ps and no more than $\sim 20$ atoms. This allows the simulation of direct scattering. But it is to be expected that adsorption and scattering after absorption needs longer timescales [3, 23]; the modeling of the $\mathrm{Au}(111)$ surface reconstruction [32-35] for 
scattering simulations appears also unfeasible with AIMD. Propagating classical trajectories on a previously constructed PES has the advantage of making trajectory calculations many orders of magnitude faster than employing AIMD and would also allow for much larger cell sizes and longer simulation times. But, as signified previously, the construction of a multidimensional PES is far from elementary. Additionally, a PES, being in its nature adiabatic, provides no information on electronic transitions.

In this work, I not only show the construction of a multidimensional potential energy surface based on the analytic form of the effective medium theory (EMT) [2, 21, 23, 36-38] for $\mathrm{H}$ on $\mathrm{Au}(111)$, but I also show how electronically nonadiabatic effects can be included on the level of LDFA. I have fitted the EMT expression to ab initio energies of $\mathrm{H}$ and $\mathrm{Au}$ atoms where the $\mathrm{H}$ atom positions are varied in and above the $\mathrm{Au}(111)$ surface while the $\mathrm{Au}$ atoms are frozen to their relaxed $f c c$ positions, providing information dependent on the $\mathrm{H}$ atom position. The input data set further included such energies taken from a single AIMD trajectory where all atoms move, sampling the configuration space in regions relevant for the Au atom motion during a trajectory. Compared with numerical potentials, the EMT analytic equations have been built to reproduce the physical behavior of metal surfaces [2], which makes it possible to describe the potential energy at positions away from the relaxed Au lattice position without requiring a large input data set. The fitting root-mean-square (rms) error for the energy ( $\sim 150 \mathrm{meV})$ is on the order of the deviation found between different GGA-functionals for the surface adsorption sites [39] and smaller than the systematic error of GGA-DFT [40]. Furthermore, EMT provides the background electron density for all positions taken up by the $\mathrm{H}$ atom and therefore allows the implementation of LDFA in form of the Langevin equation in a self-consistent manner [22].

I used this potential energy surface to perform molecular dynamics (MD) simulations with a self-written program package to which I contributed substantially that includes the fitting program, the MD-procedure and an implementation of the analytic expressions for the forces of the EMT. I found that, if the energy loss to electron hole pairs is accounted for, it is a much more efficient pathway than the energy loss to phonons. Furthermore, ehp excitation governs not only the dynamics of scattering but also those of sticking, adsorption and absorption behavior. Mechanisms for sticking behavior can be derived. I studied the scattering behavior of $\mathrm{H}$ from $\mathrm{Au}(111)$ for a number of different incidence conditions, including different surface temperatures, incidence energies, incidence angles and surface structures.

The simulation of the $22 \times \sqrt{3}$ reconstruction pattern of the $\mathrm{Au}(111)$ surface shows that it is stable up to at least $700 \mathrm{~K}$, energetically favorable compared to adatoms on the surface, but not compared to the unreconstructed surface as has been observed in DFT simulations [41-43]. The present PES reproduced experimentally observed geometrical properties of the reconstruction [32.

Comparison to experiment shows that the prediction for energy loss made with the inclusion 
of energy loss to ehp is capable of explaining the experimentally observed energy loss behavior, and good agreement between theory and experiment is achieved [44]. Furthermore, I was able to rationalize the apparent lack of an isotope effect that has been observed experimentally. 


\section{Theoretical Methods}

\subsection{The Born-Oppenheimer Approximation}

Most of the discussion here follows along the lines of Tully [45] and Tannor [46], supplemented by [47, 48]. The time-independent nonrelativistic Schrödinger Equation in full space of the electron and nuclear coordinates is:

$$
\hat{H} \Psi(\mathbf{r}, \mathbf{R})=E \Psi(\mathbf{r}, \mathbf{R})
$$

where $\Psi(\mathbf{r}, \mathbf{R})$ is the wavefunction in full coordinate space of nuclei and electrons, $\hat{H}$ is the Hamilton-operator :

$$
\begin{gathered}
\hat{H}=-\sum_{I} \frac{\hbar^{2} \nabla_{\mathrm{N}, I}^{2}}{2 M_{I}}+\sum_{I<J} \frac{Z_{I} Z_{J} e^{2}}{\left|\mathbf{R}_{I}-\mathbf{R}_{J}\right|}-\sum_{i} \frac{\hbar^{2} \nabla_{\mathrm{e}, i}^{2}}{2 m_{\mathrm{e}}}+\sum_{i<j} \frac{e^{2}}{\left|\mathbf{r}_{i}-\mathbf{r}_{j}\right|}-\sum_{i, J} \frac{Z_{J} e^{2}}{\left|\mathbf{r}_{i}-\mathbf{R}_{J}\right|} \\
\hat{H}=\hat{T}_{\mathrm{N}}+\hat{H}_{\mathrm{e}}, \quad \hat{H}_{\mathrm{e}}=\hat{V}_{\mathrm{N}}+\hat{T}_{\mathrm{e}}+\hat{V}_{\mathrm{e}}+\hat{V}_{\mathrm{N}, \mathrm{e}}
\end{gathered}
$$

$\hbar$ is Planck's constant divided by $2 \pi$. $\mathbf{R}$ and $\mathbf{r}$ refer to the positions of the nuclei and electrons respectively, and $M$ and $m_{\mathrm{e}}$ to their masses. The capital indices are associated with the nuclei and the lower case indices with the electrons. $Z_{I}$ denotes the nuclear charge of the nucleus $I$ and $e$ the elementary charge. The terms in Eq. 2.3 correspond to the kinetic energy operator $\hat{T}_{\mathrm{N}}$, the electronic Hamiltonian $\hat{H}_{\mathrm{e}}$, the potential energy operator $\hat{V}_{\mathrm{N}}$ of the nuclei, the kinetic energy operator $\hat{T}_{\mathrm{e}}$ and the potential energy operator $\hat{V}_{\mathrm{e}}$ of the electrons, and the potential energy operator of the interaction between electrons and nuclei $\hat{V}_{\mathrm{N}, \mathrm{e}}$.

The total electron-nuclear wavefunction can be expanded in terms of electronic basis functions:

$$
\Psi(\mathbf{r}, \mathbf{R})=\sum_{n=0}^{\infty} \psi_{n}(\mathbf{r} ; \mathbf{R}) \chi_{n}(\mathbf{R})
$$

The sum runs over all electronic states (but for the unbound electronic states it should really be an integral). $\chi_{n}(\mathbf{R})$ is the nuclear wave function that corresponds to the motion of the nuclei along the potential energy surface of the electronic state $n[45] . \psi_{n}(\mathbf{r} ; \mathbf{R})$ represents the eigenfunction of the electronic state $n$

$$
\hat{H}_{\mathrm{e}} \psi_{n}(\mathbf{r} ; \mathbf{R})=E_{n} \psi_{n}(\mathbf{r} ; \mathbf{R})
$$


which depends only parametrically on the positions of the nuclei. $E(\mathbf{R})$ is the energy eigenvalue of the electronic Hamiltonian Eq. (2.3) for a state $n$. Substitution of Eq. (2.4) into Eq. (??), multiplying from the left with $\psi_{m}(\mathbf{r} ; \mathbf{R})$ and integrating over coordinates of the electrons results in the expression for the nuclear wavefunction:

$$
\begin{aligned}
\sum_{n} \hat{H}_{m, n}(\mathbf{R}) \chi_{n}(\mathbf{R}) & =-\frac{\hbar^{2}}{2 M_{I}} \sum_{n}\left\{\left(\hat{T}_{\mathrm{N}}+E_{n}(\mathbf{R})\right) \delta_{m, n}+2 \hat{T}_{m, n}^{(1)}(\mathbf{R})+\hat{T}_{m, n}^{(2)}(\mathbf{R})\right\} \chi_{n}(\mathbf{R}) \\
& =E_{m} \chi_{m}(\mathbf{R})
\end{aligned}
$$

where

$$
\begin{aligned}
& T_{m, n}^{(1)}=\left\langle\psi_{m} \mid \nabla_{\mathrm{N}} \psi_{n}\right\rangle \\
& T_{m, n}^{(2)}=\left\langle\psi_{m} \mid \nabla_{\mathrm{N}}^{2} \psi_{n}\right\rangle
\end{aligned}
$$

The angle braket $\langle\ldots\rangle$ correspond to integration over all electronic coordinates.

The off-diagonal elements of $T_{m, n}^{(1)}$ and $T_{m, n}^{(2)}$ describe the nonadiabatic interactions that promote transitions between the electronic PESs. $T_{m, n}^{(1)}$ is the dominant of the two. Because $T_{m, n}^{(1)}$ is anti-Hermitian (that is $T_{m, n}^{(1)}=-T_{n, m}^{(1) *}$ ), choosing the electronic wavefunctions to be real-valued will result in the disappearance of its diagonal elements. If seen from the perspective of the electrons, the nuclei are approximately stationary (the higher their mass, the more stationary they would appear). The dynamics of electrons and nuclei can be separated to both nuclear and electron differential operators when electrons and nuclei have similar momenta, for then electrons move much faster (on much smaller timescales) than the nuclei, which is a result of the large mass difference between them. $T_{m, n}^{(1)}$ off-diagonal contributions are proportional to the mass ratio of electrons and nuclei $\left(\propto \sqrt[4]{m_{\mathrm{e}} / M_{I}}[49]\right)$ and so small that they can usually be neglected. $T_{m, n}^{(1)}$ however enters Eq. 2.6, so if the velocities of the nuclei are large, this term can lead to significant contributions. Eq. 2.8) can also be regarded as a very small contribution due to the difference of electron and nuclear masses. The neglect of the terms associated with Eq. 2.7) and (2.8) gives rise to the Born Oppenheimer Approximations:

$$
\hat{H}(\mathbf{R}) \chi_{n}(\mathbf{R})=\left(\hat{T}_{\mathrm{N}}+E_{n}(\mathbf{R})\right) \chi_{n}(R)=E_{n} \chi(\mathbf{R})
$$

\section{Failure of the Born-Oppenheimer Approximation}

To say that the BOA is universally applicable would be folly; from Eq. 2.6 it is clear that it will fail if Eq. 2.7) or (2.8) become too large. Such cases could be imagined to be that the velocity of the nuclei is so high that the second last term $\hat{T}_{m, n}^{(1)}$ on the right hand side of Eq. 2.6 has a significant contribution, for example in high energy collision. Another potential cause for breakdown of the BOA would be that the separation between electronic states of a system is not large enough: if they are too close, only a small shift of the nuclear positions would cause 
a significant change to the electronic eigenstate; or that indeed electronic energy levels come to cross one another, for example during electron transfer [50].

The adiabatic representation retains the ordering of energy levels. That means that, when electronic energy levels cross, the adiabatic representation depicts them as an avoided crossing of a state of higher and lower energy and the electronic wavefunction changes its character drastically in the region of the avoided crossing. If, for example, a gas-phase diatomic molecule dissociates, before the dissociation, the adiabatic ground state will correspond to that of a covalent bond, while after the dissociation, the ground state will describe something entirely different, e.g. two separate atoms. The terms involving Eq. 2.7) can then be quite large due to the sudden change in the nature of the ground state wavefunction [46, 51].

In general, the adiabatic approximation becomes suspicious when the PESs of any electronic states come within a few tenths of an electron volt [45]. Massey [52] introduced a criterion that enables estimating whether nonadiabatic effects might play a role in a collision between two particles. This criterion depends on the energy separation $\Delta E$ between the initial state $\mathrm{A}$ and a possible excited state $\mathrm{B}$, as well as the relative velocity of the colliding particles; the larger the energy separation and the lower the relative velocity, the more likely the adiabatic approximation will hold.

On metal surfaces, electronic states are not well separated but form a continuum. If the electrons of the particle interact with those of the metal, then energy transfer between the molecule and the surface could occur via excitation and deexcitation of electron hole pairs. Over the years there have been numerous experimental demonstrations of for the failure of the BOA when particles interact with metal surfaces, perhaps starting with Thompson's observation in 1905 of negative particle emission in the wake of gas adsorption on alkali metal surfaces [53]. A prominent example of the BOA failing when a molecule approaches the surface is the scattering of nitric oxide off metal surfaces [14, 50, 51]. When NO is scattered off metal surfaces, the vibrational excitation probability shows an Arrhenius dependence on the surface temperature, as well as a dependence on the incidence energy without, however, showing a kinetic energy threshold to vibrational excitation. The combination of both are a strong indication that the energy necessary for the vibrational excitation can be taken from the metal surface, which was further supported by the observation that vibrational excited NO was also deexcited when scattering off metal surfaces [14]. The large mismatch between frequencies of the vibrational modes of NO and those of the phonons disqualifies interaction with phonons as an effective energy dissipation channel. The deexcitation of NO can be pictured using the vibrational autodetachment mechanism [51]: when the vibration of a highly-excited NO is close to its outer turning point, an electron transfers from the metal to the NO. At the inner turning point of the ionic $\mathrm{NO}^{-}$-vibration, the presence of an electron on the molecule is highly unfavorable energetically, the potential energy of the electron on the molecule greatly exceeds the Fermi 
level. It is then possible for the electron to hop back to the metal into an occupied state high above the Fermi-level, leaving a deexcited NO molecule [14, 50]. For scattering of highly vibrationally-excited NO from low work function metals, electron emission can be observed [13].

Interactions between electronic states of a molecule and a metal surface have been studied by comparing the lifetimes of vibrational modes of adsorbates on metal surfaces to those of adsorbates on insulator surfaces. The relaxation of carbon monoxide vibrations on copper surfaces, for example, has been observed to be on the ps-timescale [54], whereas CO adsorbed on $\mathrm{NaCl}(100)$ exhibits a lifetime of $\mathrm{ms}$ [51, 55]. The molecular vibrations of $\mathrm{CO}$ couple only weakly and ineffectively to phonons since the frequencies of the modes are much higher than those of the phonon modes and thus far out of resonance. For CO on metal surfaces, the relaxation is dominated by charge transfer between the metal and the resonant antibonding $2 \pi^{*}$ orbital of the $\mathrm{CO}$; the tail of this orbital dips into the Fermi-level of the metal so that the resonant state is periodically filling and emptying as the molecule vibrates on the surface [56, 57].

One further example of the BOA breakdown in atom-surface reactions involves the detection of chemicurrents in recombining reactions following the Langmuir-Hinshelwood mechanism when $\mathrm{H}$ atoms impinge on Au metal-insulator-metal structure. Here, excess energy from the reaction is dissipated into ehp excitation [58, 59].

\subsection{Potential Energy Surfaces}

Using Eq. 2.5), given a fixed position of the nuclei, it is possible to calculate the energy of a system. It allows one to gain information on how a system develops over time. In molecular dynamics (MD) simulations, the classical equations of motion are solved numerically in a stepby-step manner (which is only valid if the particle positions varies smoothly with time) where the positions, velocities and accelerations the atoms in the system are predicted after a discrete time step $\delta t$. Then, the forces and hence the accelerations at $t+\delta t$ are calculated from the new positions, the predicted positions (velocities and accelerations) corrected by making use of the new accelerations and so on. Most importantly for this purpose is that the forces need to be calculated in each time step [60, 61. There are at least two ways to set about solving this problem. In the first, rather straight forward way named ab initio molecular dynamics [28, 29], one can evaluate Eq. 2.5) and determine the forces from it "on the fly", meaning in every single time step of a trajectory. Accounting for changes in the nature of bonds will not provide any problems but those intrinsic to the chosen theoretical method and AIMD only requires such preparations that in any case are necessary to perform the electronic structure calculations. On the other hand, full electronic structure calculations are usually very expensive in terms of computational cost and calculating a single trajectory might include several hundreds of steps (see e.g. Kroes et al. [3]). The simulation times and system sizes accessible with AIMD are 
therefore limited: the computational workload for calculating a structure scales roughly with $N^{3}$ [62] with increasing system size $N$; if $10^{M}$ is the number of molecular dynamic steps (i.e. electronic structure calculations) per trajectory and $10^{n}$ is the number of trajectories that one roughly needs to average over different initial conditions, then approximately $10^{M+n}$ electronic structure calculations are required in total 63$]$.

The other approach for molecular dynamic simulations is to map out the potential energy surface of the electronic state $n$ under consideration by calculating the potential energy as a function of the nuclear positions. By interpolating between the calculated points with analytic functions, one obtains an analytic potential energy surface, and a mathematical model of the energy landscape. That means that one replaces the solution of Eq. 2.5 with a simplified energy expression based either on physical considerations and reasonable approximations, or very flexible mathematical functions without physical meaning [64]. The major disadvantage of analytic PESs is that calculating the input data set from electronic structure calculations may take much time, therefore, mathematical models that require few input points to describe large parts of the potential energy landscape would be preferable. Furthermore, for an analytic PES, the input data set needs to be fitted to the mathematical model which may also prove rather cumbersome, especially if the mathematical model contains many parameters. Such an analytic PES describes one system only, e.g. hydrogen in interaction with gold, and would need to be constructed anew for very similar problems, e.g. hydrogen on palladium. To make an analytic PES consequently advantageous in comparison to the AIMD approach, not only does calculating the data set that goes into the built up of a PES and performing the fitting has to take less time and effort than calculate a set of AIMD trajectories for a given problem. But the evaluation of the analytic equation for the PES have to require a minimal computational effort compared to the electronic structure calculations that AIMD needs to evaluate Eq. 2.5] on the fly [47]. If successful, solving the equations of motion using PESs can be several thousand times faster than AIMD calculations (as shall be seen below), therefore making it possible to go to much larger system sizes, time scales and number of trajectories. However, computation time is not the only crucial test for PESs: for in the area of the PES that a trajectory would probe, to be reliable, the analytic PES has to provide results that are of the same order of accuracy as the electronic structure methods on which the PES is based upon [5, 65].

Building an accurate analytic PES includes careful weighing between the accuracy of the PES and the computational effort. That encompasses also the question if it is necessary to include all degrees of freedom or whether it is sufficient to treat just those that contribute majorly to the process under consideration. For example, if a diatomic molecule scatters non-reactively from the surface, do we need to consider the motion of the surface atoms and that of the molecule or is it sufficient to deal with a six-dimensional potential energy surface that encompasses all degrees of freedom of the molecule, but includes none of the surface [6]? 
Perhaps the most difficult choice in building a PES is the choice of its mathematical description. In interactions of mono- or diatomic particles at metal surfaces, the choice of mathematical model is usually between fitting the potential energies and the corresponding nuclear coordinates with flexible mathematical functions of machine-learning origin like Neural Networks [64, 65], the corrugation reducing procedure (CRP) [66] and modified Shepard method (MS) [67, 68, or functions that enforce some of the physical properties of the system like London-Eyring-PolanyiSato potential [69, 70] or the many body expansions like e.g. Effective Medium Theory [23, 38] or the embedded atom method (EAM) [71, 72. The following section shall therefore give a short overview over these choices for potentials, starting with the flexible mathematical functions.

When treating single atoms scattering adiabatically from a metal surface, interaction with phonon motion would be the only way for a particle to lose its energy upon impact. Fortunately, most modern methods allow the treatment of surface degrees of freedom. For single atoms in interaction with metal surfaces, many body potentials have been used [73-75], e.g. to study the self-diffusion of $\mathrm{Cu}$ [73], the corrugation reducing procedure was employed [76-78] and Morsetype potentials can be used to study gas-surface interactions e.g. [69].

Neural Network potentials are built up of non-linear flexible mathematical functions without physical meaning that do not make any assumptions about the functional form of the underlying problem or make any approximations. They are a very general fitting tool that can, in principle, represent any real-valued function and can fit the input data set up to very high accuracy [64, 79, 80]. As any other expression for a PES, they provide upon input of atomic position the corresponding potential energy and they are fitted to the result of electronic structure calculations of one method [64]. They were first used to create low-dimensional PESs of molecules in interaction with surfaces by Blank et al. for $\mathrm{CO}$ on $\mathrm{Ni}(111)$ [81] as a proof of principle, and by Lorenz et al. with $a b$ initio energy values as input data set in 2004 [80 for a hydrogen molecule on a $\operatorname{Pd}(100)$ surface.

Due to their great flexibility they can incorporate the change of bond type (or even dissociation) and are not limited by the type of interaction while capable of treating all degrees of freedom in small systems. On the other hand, they become very costly for larger system sizes, their evaluation is notably slower than classical force fields and, due to their lack of physical basis, extrapolating to areas outside the region of the training set can lead to very large errors. Their construction is far from effortless and needs a large number of training points from electronic structure calculations [64]. For example, the fitting of a global, full-dimensional potential energy surface for copper that included different cluster sizes, surface structures and defects, Artrith et al. [82] were able to obtain a root-mean-square error of below $10 \mathrm{meV}$ but had to employ an elaborate correction to make the Neural Network PES independent of the number of atoms used in a given simulation. Even though they used an additional efficient procedure aimed at reducing the number of electronic structure calculations, they needed about 38000 
DFT-reference calculations as input for the construction of the PES, plus another 3800 as independent testing set. If the Neural Networks are chosen too large, overfitting might occur so that structures from the training set are well represented, but the energy for atomic configurations in between training points can deviate significantly [82].

The Modified Shepard Interpolation Scheme developed by Collins et al. [67, 68] is a local expansion around geometries where the energy and other properties have explicitly been calculated. This means that in this method, only the region is mapped out that is expected to contribute to trajectories during MD calculations. It is therefore not a method suited to obtain a global potential energy surface but rather one that is specialized on getting good results for reaction dynamics. Its greatest advantage is that in this respect, it needs fewer input points than a global PES and is capable of describing polyatomic adsorbates, reactive and nonreactive collisions, physisorption and chemisorption and reaction at surfaces. It has not yet been possible to include the effects of surface motion directly [83. The potential energy at any given point is obtained as a weighted average second-order Taylor series for and from each data point of the configuration space (or just from those in the vicinity). Since the functions have no predefined symmetry, the symmetry needs to be included explicitly. The PES is built by starting with a few points in a region that is deemed important for the process that is considered. Then, classical trajectories are run on the PES and compared to an observable like e.g. dissociation probability. If the PES does not give results for this observable within a certain tolerance, points from $a b$ initio electronic structure calculations are added into the regions that are important for the accurate determination of the potential until the observable is converged [84]. For a 6-D potential of $\mathrm{H}_{2}$ interacting with $\mathrm{Cu}(111)$, the MS does poorly for regions that are not considered relevant for dynamics, but does well for barrier heights [5].

Busnengo et al. [66] introduced the Corrugation Reducing Procedure in 2000 for diatomic molecules in interaction with surfaces. It is an interpolation method that reduces the corrugation of the strongly repulsive part of the PES by subtracting the atom-surface potentials from the total energy and fitting the less corrugated residual PES with suitable analytic functions. The interpolation function can e.g. consist of cubic spline interpolation over Fourier series over trigonometric functions. This method provides a highly accurate PES [5, 66, 79]. For example, constructing a potential for $\mathrm{H}_{2}$ at $\mathrm{Cu}(111)$, the accuracy for reproducing data that was not included into the fit lies around $30 \mathrm{meV}$, reproducing also the orientation of the molecule predicted by the GGA-DFT input calculations [5]. It, too, has a frozen surface approach and can be improved by increasing the input data set of $a b$ initio calculations to which the CRP is fitted, and has been used together with neural networks [85]. Extending it beyond the frozen surface approach is almost unfeasible [5, 84], however, but other methods can be coupled with it to include energy dissipation to phonons, e.g. via the surface oscillator model in which the entire surface can move upon interaction with the impinging particle [6] or by describing the particle- 
surface interaction with the CRP-procedure and treating the motion of the surface-atoms in a pair-wise fashion [77].

It allows the inclusion of zero-point energy [86 88] and has been used to describe single atoms in interaction with metal surfaces [89, 90], but mostly used to built 6-D PES to describe $\mathrm{H}_{2}$ interacting with usually frozen metal surfaces $\left(\mathrm{H}_{2} / \mathrm{Pd}(111)\right.$ [87, 88, $\mathrm{H}_{2} / \mathrm{W}(100)$ and $\mathrm{H}_{2} / \mathrm{W}(110)$ [91], $\mathrm{H}_{2} / \mathrm{Pd}(110)$ [92], $\mathrm{H}_{2} / \mathrm{Ni}(110)$ [93], $\mathrm{H}_{2} / \mathrm{Pt}(211)$ [94], $\mathrm{H}_{2} / \mathrm{Pd}(100)$ [95], activated systems $\mathrm{H}_{2} / \mathrm{Ni}(100) \quad$ and $\mathrm{H}_{2} / \mathrm{Ni}(111)$ [93], $\quad \mathrm{H}_{2} / \mathrm{Cu}(100)\left[96, \quad \mathrm{H}_{2} / \mathrm{Cu} / \mathrm{Ru}(0001) \quad\right.$ and $\mathrm{H}_{2} / \mathrm{Pd} / \mathrm{Ru}(0001)$ [86], $\mathrm{H}_{2} / \mathrm{NiAl}(110)$ [90, $\mathrm{H}_{2} / \mathrm{Cu}(110)$ [97] and $\mathrm{H}_{2} / \mathrm{Ru}(0001)$ [98]).

The London-Eyring-Polanyi-Sato (LEPS) potentials are based on physical considerations and use Morse-potential-like formulations. They were developed for tri-atomic interactions in gas phase [69, 99]. In 1975 McCreedy and Wolken introduced the treatment of gas-surface interaction for LEPS for the interaction between $\mathrm{H}_{2}$ and $\mathrm{W}(001)$ [70] which includes the surface periodicity, but lacks flexibility [79]. Here, again, the motion of the surface atoms is not taken into account but the surface is instead modeled as a rigid background potential that is periodic in the plane of the surface. The energy exchange between surface and particle can indirectly be taken into account, e.g. in form of generalized Langevin oscillators [100]. The problem is divided into two body fragments whose interaction is assumed to be describable via Morse potentials [70]. LEPS potentials have been extensively used to describe dissociative molecular adsorption and Eley-Rideal recombination [101] and have been extended to include a dependence of the Morse parameters on the in-plane positions of the particle to reproduce the surface periodicity (PLEPS). They have been extended further to be able to treat more complex energy landscapes by including functions without physical meaningfulness. In general, the LEPS formulation and its modifications require little input data, relying on 300 to 500 input values but is limited in its accuracy [101, 102], and can describe one and two-atomic adsorbates but have primarily been used to describe the interaction of diatomic molecules with surfaces [103]. It appears to be impossible to treat interactions with this potential where particles enter the surface.

In non-reactive dynamics, Potential Energy functions or force fields make it possible to describe the energy or forces from an arrangement of the position of the atoms that compose the problem. Classical force fields are usually built up as sums over many simpler potential energy expressions that mimic the problem [104]. The great advantage of force fields is that they allow all atoms to move: they are mainly made of pair potentials without any symmetry assumption which allows the treatment of disordered and complex surface structures. Force fields can not only be used to describe the approach of atoms or molecules to each other or surfaces, but are also capable of describing reactions (e.g. formation and dissociation of hydrocarbons [105] or silicon bond breaking [106]). The assumption behind these so called bond-order based potential force fields is that the strength of a chemical bond depends on its environment as suggested 
by Linus Pauling's bond order theory which allows the treatment of more complex molecules in interaction with surfaces. For example, in the Reactive Force Field approach the energy resulting from the bond between the atoms is described as a sum of pair potentials between all atoms of the surface. Although Reactive Force Fields supply full-dimensional potential energy surfaces, the description of the modification of the electronic structure when bonds are formed or broken is difficult and limits the application of reactive force fields [101]. They can have large parameter sets, e.g. 77 parameters and a rather large database of input electronic structure energies $\left(10^{4}\right)$ in the case of $\mathrm{CH}_{4}$ on $\mathrm{Pt}(111)$ and $\mathrm{Ni}(111)$ and are applicable for a precisely defined region of conditions. Its parameters are usually not physically meaningful [107].

Another method for the description of potential energy function, and going beyond the pairwise potential form employed in many force field potentials, are the many-body expansion formulations. In this respect, the embedded atom method (EAM[71, 72, 108]) and the effective medium theory (EMT [2, 36-38]), both developed to describe metal systems in interaction with one another and metal systems in interaction with hydrogen atoms, have been applied to construct a number of potential energy surfaces. The EMT shall be dealt with in greater detail in section 2.4 having been used to construct the potential energy surface discussed in this thesis. The great advantages about many-body potentials like EAM or the closely related EMT is that these methods have been developed to describe a certain type of system as a density functional theory of their own, in case of the EMT, first chemisorption of single atoms (e.g. hydrogen) to a host [36, 37] and later the ground state properties of fcc-transition metal surfaces with and without interaction with hydrogen, solids and phonon-spectra [2, 38, 101]. This means that they already include most of the physical properties of the system, allow motion of surface and adsorbate atoms and have a very small number of parameters (only seven per atomic species in case of the EMT theory, which, with a hydrogen atom interacting with a gold surface, amounts to fourteen parameters in total). All this results in fits only needing a very small number of electronic structure calculations (below $10^{3}$ ) to produce accurate PESs. Having analytic equations, they make the solution of Newton's equations of motion straight forward, and being developed as density functional theory methods also provide self-consistently background electron densities which can be used to account for nonadiabatic effects in terms of electronic friction [22]. Furthermore, these potentials are not limited in the temperature range they can describe accurately (as long as the surface stays solid) and, the forces being easily computable, allow the treatment of large unit cells which make it possible to deal with the effects of ad-atoms on surfaces, steps and, even more interestingly, complex reconstruction patterns like the $\mathrm{Au}(111)$ (herringbone-) surface reconstruction. On the downside, using these methods limits the systems that can be treated: going beyond metal surfaces is impossible and treating less metal-like atoms or even molecules may prove difficult, although attempts to treat diatomic molecules and excited states with EAM have been made (EDIM method) [109]. 


\subsection{Density Functional Theory}

The information for this section were taken from Ref. [62, 110, 111].

To obtain the energy $E_{0}$ of the ground state and the ground state wavefunction $\psi_{0}(\mathbf{r} ; \mathbf{R})$, one needs to solve the electronic Schrödinger equation Eq. 2.5. Unfortunately, for many body systems, this is not feasible. The standard quantum chemistry approach to such a problem is to apply the variational principle which states that the energy of any trial wavefunction will be an upper bound of the true ground state energy of the system

$$
\frac{\left\langle\psi_{k}\left|\hat{H}_{\mathrm{e}}\right| \psi_{k}\right\rangle}{\left\langle\psi_{k} \mid \psi_{k}\right\rangle} \geq E_{0} .
$$

and equality will only be reached when $\psi_{k}=\psi_{0}$. The denominator on the l.h.s of Eq. 2.10) will be 1 when the electronic wave functions are normalized. This means that, the lower the energy value obtained with an electronic guess wave function, the closer this wave function will be to the ground state electronic wave function, therefore offering a tool to judge how good a guess wave function is.

Methods to evaluate the wave function depend on which approximations are made to the Hamiltonian to make it calculable. The only information that is needed for the construction of the electronic Hamiltonian for a given system is the number of electrons $N_{e}$ of this system and the potential $V_{\mathrm{N}, \mathrm{e}}$ which in turn is determined by the nuclear charge $Z$ and the positions of the nuclei. With these information, the ground state wave function can, in principle, be calculated and the energy of the system can be determined. The number of electrons is in direct relation to the electron density $n(\mathbf{r})$ of the system where $\mathbf{r}$ defines the position from where the electron density is taken:

$$
N_{\mathrm{e}}=\int \mathrm{d} \mathbf{r} n(\mathbf{r})
$$

Furthermore, the density has also cusps at the nuclei position, therefore, the positions of the nuclei are derivable from the electron density. Additionally, the density at the position of the nuclei contain information about the nuclear charge. This means that the electron density in fact contains all information one needs to construct the electronic Hamiltonian for a system. Instead of calculating the energy of a system from the wave function, which depends on the positions of all the electrons in the system (and their spin), the electron density could be used which depends only on three coordinates.

All density functional theory starts with the Hohenberg-Kohn theorem which states that the ground state of a given system has only one specific electron density associated with it. This ground state electron density $n_{0}(\mathbf{r})$ uniquely defines the system's Hamiltonian and with that makes it possible to calculate any observable of the system. Secondly, the energy of the ground state also has variational property with respect to the electron density. This means that the 
electron density can be used in lieu of the wave function to calculate a system's energy. The ground state energy of a system can therefore be expressed as a functional

$$
E_{0}\left[n_{0}\right]=T_{\mathrm{e}}\left[n_{0}\right]+V_{\mathrm{ee}}\left[n_{0}\right]+V_{\mathrm{N}, \mathrm{e}}\left[n_{0}\right]=F\left[n_{0}\right]+V\left[n_{0}\right] .
$$

$T_{\mathrm{e}}$ and $V_{\text {ee }}$ are universal functionals, they can be combined to the Hohnberg-Kohn functional $F$ that, if it were known, would be a universal key to all imaginable systems. $V_{\mathrm{N}, \mathrm{e}}$ where $\mathrm{I}$ have dropped the indices on the right hand side of Eq. 2.12 is an external potential that varies for different systems.

The electrostatic interaction energy $V_{\text {ee }}$ between the electrons can be split up into the Hartree energy $V_{\mathrm{H}}$ whose functional form is known and a nonclassical contribution $E_{\text {ncl }}$ to the electronelectron interaction that contains a self-interaction correction (the interaction of the electron density with itself in the Hartree energy), exchange and Coulomb correlation:

$$
V_{\mathrm{ee}}[n]=\frac{1}{2} \iint \mathbf{d r d r}^{\prime} \frac{n(\mathbf{r}) n\left(\mathbf{r}^{\prime}\right)}{\left|\mathbf{r}-\mathbf{r}^{\prime}\right|}+E_{\mathrm{ncl}}[n]=V_{\mathrm{H}}[n]+E_{\mathrm{ncl}}[n]
$$

In 1965, Kohn and Sham [112] suggested an approach to treat the universal functional $F$ that avoids the shortcomings in treatment of the kinetic energy functional of direct methods such as the Thomas-Fermi-method. In the Kohn-Sham approach, a part of the kinetic energy functional is treated in terms of single-particle orbitals (i.e., one-electron functions) $\phi(\mathbf{r})$ of a noninteracting system. This allows to treat a large part of the kinetic energy functional exactly. For it, the kinetic energy functional is decomposed into a part $T_{\mathrm{s}}[n]$ (s for single-particle) that represents the kinetic energy of individual, noninteracting particles and the remainder $T_{\mathrm{c}}[n]$ (c for correlation).

$$
T[n]=-\frac{\hbar^{2}}{2 m_{\mathrm{e}}} \sum_{i}^{N_{\mathrm{e}}}\left\langle\phi_{i}(\mathbf{r})\left|\nabla_{i}^{2}\right| \phi_{i}(\mathbf{r})\right\rangle+T_{\mathrm{c}}[n]=T_{\mathrm{s}}[n]+T_{\mathrm{c}}[n]
$$

$T_{\mathrm{c}}$ and $E_{\mathrm{ncl}}$ are combined into the exchange correlation functional $E_{\mathrm{xc}}$ so that the total energy expression assumes the following form:

$$
E[n]=T_{\mathrm{s}}[n]+V_{\mathrm{H}}[n]+E_{\mathrm{xc}}[n]+V[n]
$$

The single particle orbitals are chosen such that they reproduce the density of the original system:

$$
n(\mathbf{r})=\sum_{i}^{N_{\mathrm{e}}}\left\langle\phi_{i} \mid \phi_{i}\right\rangle
$$

To obtain the ground state energy of the system, the variation principle can be applied:

$$
0=\frac{\delta E[n]}{\delta n(\mathbf{r})}=\frac{\delta T_{\mathrm{s}}[n]}{\delta n(\mathbf{r})}+\frac{\delta V_{\mathrm{H}}[n]}{\delta n(\mathbf{r})}+\frac{\delta V_{\mathrm{N}, \mathrm{e}}[n]}{\delta n(\mathbf{r})}+\frac{\delta E_{\mathrm{xc}}[n]}{\delta n(\mathbf{r})}=\frac{\delta T_{\mathrm{S}}[n]}{\delta n(\mathbf{r})}+v_{\mathrm{H}}(\mathbf{r})+v_{\mathrm{N}, \mathrm{e}}(\mathbf{r})+v_{\mathrm{xc}}(\mathbf{r})
$$


If Eq. 2.17) is compared with a system of noninteracting particles moving in an external potential $v_{\mathrm{s}}(\mathbf{r})$ it becomes clear that one can treat the entire problem as a pretend-noninteracting singleparticle problem where the potentials contributing to Eq. 2.17) can be seen as making up the external noninteracting-particle potential:

$$
v_{\mathrm{S}}(\mathbf{r})=v_{\mathrm{H}}(\mathbf{r})+v_{\mathrm{N}, \mathrm{e}}(\mathbf{r})+v_{\mathrm{xc}}(\mathbf{r})
$$

Eq. 2.16) and Eq. 2.18) are the Kohn-Sham equations. The Kohn-Sham orbitals $\phi_{i}$ can be obtained by solving the one-electron Schrödinger equation

$$
\left[-\frac{\hbar^{2} \nabla^{2}}{2 m_{\mathrm{e}}}+v_{\mathrm{s}}(\mathbf{r})\right] \phi_{i}=\varepsilon_{i} \phi_{i}(\mathbf{r}) .
$$

These orbitals define the noninteracting system which, according to Eq. 2.16 has the same density as the real system. Up until this point, the scheme is exact in so far that, if all functionals that make up $v_{\mathrm{s}}(\mathbf{r})$ were known, one could calculate the exact energy of the system. Unfortunately, $v_{\mathrm{xc}}$ is unknown and needs to be approximated.

An early approximation to the exchange-correlation functional was made with the local density approximation (LDA) that treats the exchange-correlation energy as that of a locally homogeneous electron gas

$$
E_{\mathrm{xc}}^{\mathrm{LDA}}[n]=\int \mathrm{d} \mathbf{r} n(\mathbf{r}) \varepsilon_{\mathrm{xc}}^{\mathrm{LDA}}(n(\mathbf{r})) .
$$

The per volume exchange of a homogeneous electron gas is known exactly and the correlation energy of a homogeneous liquid can be calculated with Quantum Monte Carlo [113 and interpolated. The LDA approximation has proven itself to be quite accurate, due mostly to systematic error cancellations: the exchange is overestimated while the correlation is underestimated. It provides reasonable geometries and vibrational frequencies but greatly overestimates atomization energies [114] and fails to predict chemical bond energies within chemical accuracy (energy errors of the order of $0.0434 \mathrm{eV}$ ). An improvement to it is the generalized gradient approximation that treats not only the local density $n(\mathbf{r})$ but also its gradient in general functions:

$$
E_{\mathrm{xc}}^{\mathrm{GGA}}[n]=\int d \mathbf{r} n(\mathbf{r}) \varepsilon_{\mathrm{xc}}^{\mathrm{GGA}}(n(\mathbf{r}), \nabla n(\mathbf{r}))
$$

The general functions can either be parametrized to test sets of selected molecules or using exact constrains (e.g. the Perdew-Burke-Ernzerhof- (PBE) [115], Perdew-Wang-'91- (PW91) [116, 117] or revised PBE (RPBE) functionals [118]). The GGA functionals do not provide chemical accuracy but provide reliable results for covalent, ionic, metallic and to some extend hydrogen bond interactions.

LDA and GGA are the first two rungs of the so-called Jacob's ladder of DFT [114] which reaches from Hartree calculation that do not treat exchange correlation effects to chemical accuracy with accurate treatment of exchange correlation effects. Further rungs (that is, further 
improvement) on this ladder include the treatment of Kohn-Sham kinetic energy densities or second derivatives of the density in the meta-GGAs. Hybrid functionals follow that mix HartreeFock exchange into the DFT exchange functional. Spin can also be dealt with in DFT by including individual functionals for the $\alpha$ and $\beta$ spin densities.

While the electron density should, in theory, contain information about all states of the system, calculating any but the ground state in DFT is not easy, for the variation principle does not apply to excited states. On the level of GGA, the description of non-local interactions such as van-der-Waals fails (although on higher levels of DFT progress has been made in that direction [119]), same as an accurate description of dative bonds cannot be achieved. Hydrogen bonds are often predicted to be too short.

\section{SRP-Functional}

Commonly used DFT GGA-functionals for gas phase particles in interaction with metal surfaces are the PBE [115], PW91 [116, 117] or RPBE functionals [118]). However, PW91 overestimates binding energies while RPBE underestimates them [39]. If functionals both over- and underestimate experimental properties, to obtain chemical accuracy for a system, the specific reaction parameter (SRP) DFT approach introduced by Chuang et al. [120] can be taken. This has been done by Diaz et al. [121] for reactive scattering of $\mathrm{H}_{2}$ from $\mathrm{Cu}(111)$. The version used in this work consists of a mixture of the PBE- and RPBE-functionals [12]:

$$
E_{\mathrm{xc}}^{\mathrm{SRP}}=x E_{\mathrm{xc}}^{\mathrm{RPBE}}+(1-x) E_{\mathrm{xc}}^{\mathrm{PBE}}
$$

with a weighting factor of $x=0.48$. The resulting functional will be referred to as 'SRP48'. Although the SRP48 has been optimized for $\mathrm{H}_{2}$ on $\mathrm{Cu}(111)$, I expect it to perform similarly well for $\mathrm{H}$ with $\mathrm{Au}(111)$, since PW91 (whose energetic behavior PBE [115] was designed to mimic) performs already quite well for $\mathrm{H}_{2}$ with Au clusters but could be improved by a mixing with RPBE [122].

\subsection{EMT}

The discussion in this section is mostly taken from the description of deriving effective medium theory in [38] and its formulation for two species [2, 22]. The effective medium theory incorporates many-body contributions to the total energy expression but requires about the same computational effort as pairwise potentials and provides the background electron density for any given atomic positions which can be readily extracted and used for the estimation of nonadiabatic effects. Its functional form is rather simple which allows the analytic calculation of the potential's derivatives with respect to the atomic positions or parameters of the potential which speeds up the calculation of the forces and fitting of the potential. 
The EMT was developed to describe the energy of an atom by embedding it into a homogeneous electron gas [36, 37] and later extended to describe $f c c$ transition metal surfaces [38] and $\mathrm{H}$ in interaction with them [2]. EMT contains many-body contributions to the energy and in the form used in this thesis is capable of describing macroscopic properties such as the bulk modulus, the cohesive energy and the elastic constants. The total energy of a system is described by a reference system, which is a perfect $f c c$ crystal and acts as an effective medium, which is corrected for the difference between real and reference system.

The basic idea behind effective medium theory is to describe the binding energy of an atom in a system by the energy it takes to embed said atom in a host of surrounding atoms. Primarily, EMT is a form of density functional theory where the approximation is made that the energy only depends on the local density. The binding energy of an atom is described by the embedding energy $\Delta E^{\text {hom }}(\bar{n})$ of embedding the atom in a homogeneous electron gas of the density $\bar{n}$. To calculate the embedding energy, first the background electron density $\bar{n}$ has to be defined. This is done by the atomic sphere approximation (ASA) where one presumes that a perfect, monoatomic solid can be divided into its neutral Wigner-Seitz (WS) cells. The WS cell can then be approximated by a perfect sphere whose radius $s_{i}$ around the atom $i$ is chosen such that the volume of the sphere equals that of a WS cell in the bulk and that the sphere remains neutral in charge. This approximation is especially viable for close-packed crystals where the WS-cell forms a complex polyhedron that almost approaches sphere-shape. The background electron density inside the sphere $s_{i}$ is then regarded as the sum over the change in electron density $\Delta n_{j}$ (induced electron densities) inside the $s_{i}$ due to embedding the neighboring atom $j$. To make it homogeneous, the electron density from the 'density tails' of the neighboring atoms are averaged over the sphere $s_{i}$.

$$
\bar{n}_{i}=\left\langle\sum_{j \neq i} \Delta n_{j}\right\rangle_{s_{i}}
$$

With this approximation, and treating the Hartree potentials that describe the electrostatic potentials similarly, the potential $v$ that contains the exchange correlation and electrostatic contributions can be written as such a one that belongs to a homogeneous electron gas for each WS cell. The embedding energy $\Delta E[n, v]$ can then be written as

$$
\Delta E[n, v]=\sum_{i} \varepsilon_{i}\left(\bar{n}_{i}\right)+\sum_{i} \Delta E_{\mathrm{T}, i}+\Delta E_{\mathrm{tail}}^{\mathrm{corr}}+\Delta E_{\mathrm{AS}}^{\mathrm{corr}}
$$

The first term in Eq. 2.24) is the cohesive energy function. The second term describes the difference between the studied system and the reference system for the one electron energy sum of the kinetic energy contribution and can be neglected for simple atoms. It is the remainder of the difference between a cell without an atom embedded into it and a cell with an atom embedded into which are otherwise collected in $\Delta E_{\text {tail }}^{\text {corr }}$. Because the charge inside $s_{i}$ must be zero, all electron density tails sticking in from the neighboring spheres must cancel out with those 
sticking out of the sphere. This leads to a complex cancellation of terms within $\Delta E_{\text {tail }}^{\text {corr }}$ making it negligible. The last term in Eq. 2.24) is a correction for the atomic sphere approximation that describes the errors from replacing the WS cells by spheres, namely that the spheres might overlap or not cover all space. $\Delta E_{\mathrm{AS}}^{\text {corr }}$ can be assumed to be just composed of its electrostatic contribution, since these are the most dominating contributions. It is composed of the difference between a system where the electron density $\bar{n}_{i}$ is calculated from the neutral sphere radius and one where the electron density $\bar{n}_{i}^{\mathrm{fcc}}$ is calculated from all the surrounding density tails. Both contain a correction that accounts for the difference between the reference system (a perfect $f c c$ crystal) and the real system. $\Delta E_{\mathrm{AS}}^{\text {corr }}$ can then be expressed as

$$
\Delta E_{\mathrm{AS}}^{\mathrm{corr}, \mathrm{es}} \approx \sum_{i} \alpha_{i}\left[\bar{n}_{i}-\sum_{i \neq j} \Delta \bar{n}_{j}^{\mathrm{fcc}}\left(r_{i j}\right)\right],
$$

where $\alpha_{i}$ are the differences in the Hartree potentials. The neighbor distance $r_{i j}$ in a perfect $f c c$ crystal with spheres of the radius $s_{0}$ (neutral sphere radius) can be written as

$$
r_{i j}=\beta s_{\mathrm{fcc}}=\frac{\sqrt[3]{\frac{16 \pi}{3}}}{\sqrt{2}} s_{0} .
$$

The electron density tail $\Delta \bar{n}$ of the neutral sphere with the radius $s$ can be expressed in an exponential form:

$$
\Delta \bar{n}(s, r)=\Delta n_{0} \mathrm{e}^{\left(\eta_{1}+\eta_{3}\right) s-\eta_{2} r}
$$

If only the twelve nearest neighbors of the perfect $f c c$ crystal are considered where the spheres are assumed to be space filling and summation only happens due to the density tail contributions into $s$, the distance $r$ in Eq. 2.25) can be replaced by $\beta s_{i}$ (see Eq. 2.26p) and the background electron density can be calculated as

$$
\bar{n}_{\mathrm{WS}}\left(s_{i}\right)=\sum_{i \neq j} \Delta \bar{n}\left(s_{i}, r_{i j}\right)=12 \Delta \bar{n}\left(s_{i}, \beta s_{i}\right)
$$

If Eq. 2.28 is combined with Eq. 2.27), $s_{i}$ can be calculated.

$$
s_{i}=-\frac{1}{\beta \eta_{2}-\eta_{3}} \ln \left[\frac{1}{12} \sum_{j \neq i} \mathrm{e}^{-\eta_{2} r_{i j}+\eta_{3} s_{j}}\right]
$$

Then, the background electron density $\bar{n}_{i}$ can be calculated as being due only to its twelve nearest neighbors:

$$
\bar{n}\left(s_{i}\right)=12 \Delta n_{0} \mathrm{e}^{-\eta s_{i}}=n_{0} \mathrm{e}^{-\eta\left(s_{i}-s_{0}\right)}
$$

where $\eta=\beta \eta_{2}-\eta_{1}-\eta_{3}$ and $n_{0}=12 \Delta n_{0} \mathrm{e}^{-\eta s_{0}} . s_{0}$ is the neutral sphere radius of a perfect $f c c$ crystal where all the atoms stay at their perfect lattice positions and thereby makes up for 
the difference between the real and the reference system. The atomic sphere correction then becomes:

$$
\Delta E_{\mathrm{AS}}^{\mathrm{corr}} \approx \sum_{i} \alpha_{i}\left[\bar{n}_{i}-\Delta n_{0} \sum_{i \neq j} \mathrm{e}^{-\eta_{2} r_{i j}-\eta_{1} s_{j}-\eta_{3} s_{j}}\right]
$$

The difference between the first and the second term in square brackets is that for the density $\bar{n}_{i}$ the assumption is made that all spheres are space filling and thus, the entire crystal can be described in terms of the radius $s$ while the second term includes the neighboring distance $r_{i j}$ instead of describing the background electron density just in terms of $s$, thereby accounting for regions that might be wrongly represented by $s$.

The dependence of the induced density on the background electron density can be neglected to avoid the self-consistency problem associated with it which results in $\eta_{3}=0$. With this, the background electron density $\bar{n}_{i}$ can be calculated as:

$$
\bar{n}_{i}=n_{0} \sum_{j \neq i} \mathrm{e}^{-\left(\beta \eta_{2}-\eta_{1}\right)\left(s_{i}-s_{0}\right)},
$$

This background electron density now serves as the connection between the real system (where the atoms are not necessarily on their perfect $f c c$ positions) and the reference system (perfect $f c c$ crystal). According to [2], $\eta_{1}=0.945 \AA^{-1}$ is a typical value for metals and will be employed here.

If the atomic sphere correction is expressed in terms of two pairwise interactions in the reference $V_{i}^{(\mathrm{ref})}\left(\bar{n}_{i}\right)$ and the real systems $V_{i j}\left(r_{i j}\right)$, a one-electron correction appears that can, however, be neglected [123. The total energy for a system consisting of $N$ atoms can be written as:

$$
E=\sum_{i=1}^{N} \varepsilon_{i}\left(\bar{n}_{i}\right)+\sum_{i=1}^{N}\left[V_{i}^{(\mathrm{ref})}\left(\bar{n}_{i}\right)+\sum_{j>i}^{N} V_{i j}\left(r_{i j}\right)\right]
$$

To treat the interaction between two different kinds of atoms (hydrogen and gold), it is necessary to use the EMT formulation for two different species of atoms which will be labeled $a$ and $b$ where a distinction between the two species is necessary. $i$ and $j$ will further serve to enumerate the atoms. The cohesive energy function $\varepsilon_{i_{a}}$ can be described by:

$$
\varepsilon_{i_{a}}=E_{0, a}\left[1+\lambda_{a}\left(s_{i_{a}}-s_{0, a}\right)\right] \mathrm{e}^{-\lambda_{a}\left(s_{i_{a}}-s_{0, a}\right)}-E_{0, a},
$$

Here, $E_{0, a}$ is the cohesive energy of the species $a$. Eq. 2.29 shows how to calculate the neutral sphere radius $s_{i_{a}}$ for the atom $i_{a}$ of the species $a$ for nearest neighbors. If the presence of a second species is included, the expressions changes to

$$
s_{i_{a}}=s_{0, a}-\frac{1}{\beta \eta_{2, a}} \ln \left[\frac{\sigma_{i_{a}}^{(a)}+\chi_{a b} \sigma_{i_{a}}^{(b)}}{12}\right] .
$$


Here,

$$
\chi_{a b}=\frac{n_{0, b} \mathrm{e}^{-\eta_{1, b} s_{0, b}}}{n_{0, a} \mathrm{e}^{-\eta_{1, a} s_{0, a}}},
$$

and $\sigma_{i_{a}}$ contains the exponential form ansatz made to describe the change in background electron density in Eq. 2.27). $\sigma_{i_{a}}^{(a)}$, same as the sum in Eq. 2.29 contains the contributions to the neutral sphere radius $s_{i_{a}}$ due to the interaction with all other atoms of the species $a . \sigma_{i_{a}}^{(b)}$ is made up from the contributions to the neutral sphere radius of the atom $i_{a}$ due to all atoms of the species $b$ summed over their number. Both contributions are calculated in the same fashion:

$$
\sigma_{i_{a}}^{(b)}=\gamma_{1, a}^{-1} \sum_{j_{b}=1}^{N_{b}} \mathrm{e}^{-\eta_{2, b}\left(r_{i_{a} j_{b}}-\beta s_{0, b}\right)} \theta_{i_{a} j_{b}} .
$$

The pairwise potential $V_{i j}\left(r_{i j}\right)$ from Eq.2.33 is calculated in a similar manner to Eq. 2.37

$$
\begin{aligned}
V_{a a} & =-\frac{V_{0, a}}{\gamma_{2, a}} \sum_{i_{a}<j_{a}} \mathrm{e}^{-\frac{\kappa_{a}}{\beta}\left(r_{i_{a} j_{a}}-\beta s_{0, a}\right)} \theta_{i_{a} j_{a}} \\
V_{a b} & =-\chi_{a, b} \frac{V_{0, a}}{\gamma_{2, a}} \sum_{i_{a}, j_{b}} \mathrm{e}^{-\frac{\kappa_{b}}{\beta}\left(r_{i_{a} j_{b}}-\beta s_{0, b}\right)} \theta_{i_{a} j_{b}} .
\end{aligned}
$$

The reference energy contribution is calculated similarly:

$$
V_{a}^{(\mathrm{ref})}=-12 V_{0, a} \sum_{i_{a}=1}^{N_{a}} \mathrm{e}^{-\kappa_{i_{a}}\left(s_{i_{a}}-s_{0, a}\right)} .
$$

Including more than nearest neighbor interactions into the energy calculations makes it necessary to include a cut-off function to allow the treatment of finite slabs during MD-simulations. A smooth cut-off function

$$
\theta_{i j}=\left[1+\mathrm{e}^{\alpha\left(r_{i j}-r_{\mathrm{c}}\right)}\right]^{-1}
$$

is used with the fall-off parameter $\alpha=\ln 10000 /\left(r_{\mathrm{r}}-r_{\mathrm{c}}\right)$ in such a manner that contributions up to the next-next-nearest neighbor distance for the $f c c$ lattice $r_{\mathrm{c}}=\beta s_{0, a} \sqrt{3}$ are included, and

$$
r_{\mathrm{r}}=\frac{4 r_{\mathrm{c}}}{\sqrt{3}+2} .
$$

The normalization coefficients $\gamma$

$$
\begin{aligned}
\gamma_{1, a} & =\sum_{m=1}^{3} x_{m}^{(a)} \mathrm{e}^{-\eta_{2, a} \beta s_{0, a}(\sqrt{m}-1)} \\
\gamma_{2, a} & =\sum_{m=1}^{3} x_{m}^{(a)} \mathrm{e}^{-\kappa_{a} s_{0, a}(\sqrt{m}-1)}
\end{aligned}
$$

are chosen to ensure that $E=0$ for the perfect $f c c$ bulk structure, with

$$
x_{m}^{(a)}=\frac{b_{m}}{12}\left[1+\mathrm{e}^{\alpha \beta s_{0, a}(\sqrt{m}-\sqrt{3})}\right]^{-1} .
$$


Each species in the EMT theory is characterized by seven parameters: $n_{0}, \varepsilon_{0}, s_{0}, \lambda, \eta_{2}, V_{0}$ and $\kappa$. Some of the parameters can be related directly to experimental data: $\varepsilon_{0}$ is the cohesive energy, $s_{0}$ is proportional to the lattice constant of the bulk via the nearest neighbor distance (Eq. (2.26) ) and $\lambda^{2}$ to the bulk modulus

$$
B=-\frac{\varepsilon_{0} \lambda^{2}}{12 \pi s_{0}}
$$

and the shear modulus can be calculated from $\eta_{2}, V_{0}, \kappa[23]$

$$
C_{44}=\frac{3 V_{0} \kappa \delta}{8 \pi s_{0}}
$$

with

$$
\delta=\beta \eta_{2}-\kappa .
$$

The other two elastic constants $C_{11}$ and $C_{12}$ can be calculated as follows:

$$
\begin{gathered}
C_{11}=\frac{3 V_{0} \delta \kappa-\varepsilon_{0} \lambda^{2}}{12 \pi s_{0}} \\
C_{12}=\frac{3 V_{0}\left(\kappa-\beta \eta_{2}\right) \kappa-2 \varepsilon_{0} \lambda^{2}}{24 \pi s_{0}} .
\end{gathered}
$$

The formulae Eq.2.46 -2.50 have been derived considering only nearest neighbor contributions. It is possible to extend them to more than nearest neighbor contributions, however resulting in much more complex formulations. The treatment not going beyond nearest neighbors leads to the relations between the elastic constants

$$
C_{11}-C_{12}=2 C_{44}
$$

which is only true for isotropic crystals (i.e. crystals whose properties and structure are in all directions the same) and has not been found to be fulfilled by gold [124]. The relation between $C_{11}$ and $C_{22}$ with the bulk modulus is found for all cubic crystals [125] to be

$$
C_{11}+2 C_{12}=3 B
$$

Jacobsen et al. 2] obtained the parameters of the EMT for several metals from experimental properties and from a self-consistent calculation of an atom embedded in a homogeneous electron gas.

In the past, EMT in the form employed here has been used to describe alloys such as $\mathrm{Ni}$ $\mathrm{Au}$ [126] and cluster in Ni-Au alloys [127] which made it necessary to refit of some of the EMT parameters to reproduce physical properties of the system like the heat of solvation. For the study of $\mathrm{Cu}-\mathrm{Mg}$ bulk metallic glasses, Bailey et al. [128, 129] obtained a new EMT-parameterization based on properties of the pure elements and intermetallic compounds from both experiment and DFT calculations. Usually, however, the parametrizations of EMT given by Jacobsen et al. [2] 
were kept, often in combination with other theoretical methods like Monte Carlo Simulations: Investigations involved the stability of surface structures [130], Pd-islands on Au(111) [131] and island decay mechanisms on $\mathrm{Au}(111)$ [132]. EMT was used to describe deformations in $f c c-$ metal surfaces [133], by Vegge et al. (e.g. [134-138]) and Rasmussen [139] for properties of screw dislocations in copper, mechanical properties of metallic nanojunctions and contacts and their breaking [140, 141], deformation of nanocrystalline $\mathrm{Cu}$ [142, 144] or cracks in fcc-metals [145], deformation of thin $\mathrm{Cu}$ films and bulk $\mathrm{Cu}[146,147]$. The formation and migration energy of vacancies was calculated for $\mathrm{Ni}$ and $\mathrm{Cu}[148,149$, and nanoparticles [150, 151]. To put it short, EMT has been used to investigate a broad range of properties of metal systems. If the EMT-parameters were changed, they were usually fitted to reproduce single physical properties important for the considerations and were taken both from experiment or calculated with DFT [127, 129, 139, 152].

Opposite to this approach, Strömquist et al. [23] considered the scattering of $\mathrm{H}$ from $\mathrm{Cu}(111)$ and fitted the parameters for the $\mathrm{H}$ atom to total energy points from DFT calculations with the PW91 functional of $\mathrm{H}$ in interaction with $\mathrm{Cu}(111)$. They found that the surface atoms do not have time to respond to the motion of the $\mathrm{H}$ atom and little difference between MD-simulations done at $0 \mathrm{~K}$ and $500 \mathrm{~K}$. Furthermore, they observed that an $\mathrm{H}$ atom that only interacts with the phonons of a system has to undergo multiple collisions to lose enough energy to stick to the metal surface, the energy transfer being rather ineffective, producing hot $\mathrm{H}$ atoms. They propose that for $\mathrm{H}$ in interaction with $\mathrm{Cu}$, the energy loss to electron hole pairs cannot be neglected, seeing as the energy loss to phonons is so very inefficient. Following this approach, we [21, 22, 44] have described the interaction of $\mathrm{H}$ on $\mathrm{Au}(111)$.

\subsection{Local Density Friction Approximation}

The local density friction approximation (LDFA) is a way to incorporate nonadiabatic effects acting on a particle during molecular dynamics simulations. It is valid if only low lying electron hole pairs are excited and the shape of the potential energy surfaces of these excited states does not deviate from that of the ground state. It treats the electronic degrees of freedom as a thermal bath through which the ionic cores move. The idea behind LDFA is that the motion of the electrons is much faster than that of the ionic cores but when the ions move through the electronic bath, then, the electrons do not necessarily adjust instantaneously to the motion of the nuclei and some electron hole pairs might be excited or deexcited. This leads to a damping of the ionic motion. As the ions move slowly, only low lying ehps need to be considered so that the entire process is nearly adiabatic. As long as the motions of the nuclei are considered classically, the total effect can be described by an extra friction force term and a stochastic term 
being added to Newton's equations of motion and thereby forming the Langevin equation [30]:

$$
m_{\mathrm{H}} \ddot{\mathbf{r}}_{\mathrm{H}}=-\nabla_{\mathrm{H}} E(\mathbf{r})-m_{\mathrm{H}} \underline{\eta} \dot{\mathbf{r}}_{\mathrm{H}}+\mathbf{F}^{\mathrm{st}}(t)
$$

Here, $m_{\mathrm{H}}$ is the mass of the $\mathrm{H}$ atom, $\mathbf{r}_{\mathrm{H}}$ is its position, $\nabla_{\mathrm{H}}=\frac{\partial}{\partial \mathbf{r}_{\mathrm{H}}}, E(\mathbf{r})$ is the total energy depending on the positions of all of the atoms, $\underline{\eta}$ is the friction tensor and $\mathbf{F}^{\mathrm{st}}(t)$ is the stochastic force and is related to the friction coefficient through the dissipation-fluctuation theorem [30, 60]

$$
\left\langle\mathbf{F}^{\mathrm{st}}(t) \mathbf{F}^{\mathrm{st}}\left(t^{\prime}\right)\right\rangle=2 m_{\mathrm{H}} k_{\mathrm{B}} T \underline{\eta} \delta\left(t-t^{\prime}\right),
$$

where $k_{\mathrm{B}}$ is the Boltzmann constant and $\delta$ is the Dirac delta function. In the Langevin equations, the stochastic force has the role of accounting for the fact that a particle undergoing Brownian motion does not come to a halt after it has dissipated its energy, but will be in thermal equilibrium with the surrounding and thus have an energy corresponding to the thermal one.

The friction coefficient $\underline{\eta}$ can be calculated in two different ways. The full-dimensional friction matrix can be calculated from transition rates between occupied and unoccupied electronic states and has $F^{2}$ tensor elements where $F$ are the degrees of freedom of the system under consideration [153].

A simpler manner [153-155] is to calculate the friction coefficient for a proton (or other heavier ions) travelling through a homogeneous electron gas. The stopping power the particle experiences can be calculated from the phase shift of the initial and scattered wave function of a free electron from the spherically symmetric potential of the proton. In turn, the stopping power can be related classically to a friction coefficient [30, 156]. This friction coefficient, from the use of a homogeneous electron gas and a spherically symmetric potential in the calculations of the phase shift, is a scalar that depends on the density of the homogeneous electron gas. Puska and Nieminen [156] have calculated the density dependent phase shifts for a proton in a homogeneous electron gas and their results from DFT-LDA calculations are in good agreement with the electronic contributions for the stopping power found experimentally. To get the background electron density dependent friction coefficient, a polynomial was used to interpolate their data [22]:

$$
\hbar \eta(\bar{n})= \begin{cases}\sum_{i=1}^{12} c_{i} \bar{n}^{i}, & \text { if } \bar{n} \leq 0.36 \AA^{-3} \\ d_{1}-\mathrm{e}^{-d_{2} \bar{n}} \cdot 10^{-3} \mathrm{meV}, & \text { if } \bar{n}>0.36 \AA^{-3}\end{cases}
$$

The coefficient for the polynomial are given in Tab.2.1.

\subsubsection{Recent Applications of LDFA}

To explain the linewidth broadening of $\mathrm{H}_{2}$-molecules vibrational modes on (jellium) metal surfaces, Hellsing and Persson [157] calculated the damping rate of a vibrating atom in a homogeneous electron gas and found that ehp in a metal substrate form an effective channel for energy 
Table 2.1.: Coefficients from the interpolation formula in Eq. 2.55 used to calculate the position dependent friction coefficient for Langevin dynamics (Reproduced and corrected from [22]).

\begin{tabular}{llll}
\hline \hline$c_{1}$ & $0.0802484 \mathrm{meV} \cdot \AA^{3}$ & $c_{2}$ & $-1.12851 \mathrm{meV} \cdot \AA^{6}$ \\
$c_{3}$ & $9.28508 \mathrm{meV} \cdot \AA^{9}$ & $c_{4}$ & $2.10064 \mathrm{meV} \cdot \AA^{12}$ \\
$c_{5}$ & $-843.419 \mathrm{meV} \cdot \AA^{15}$ & $c_{6}$ & $8853.54 \mathrm{meV} \cdot \AA^{18}$ \\
$c_{7}$ & $-48902.3 \mathrm{meV} \cdot \AA^{21}$ & $c_{8}$ & $167410 \mathrm{meV} \cdot \AA^{24}$ \\
$c_{9}$ & $-367098 \mathrm{meV} \cdot \AA^{27}$ & $c_{10}$ & $503476 . \mathrm{meV} \cdot \AA^{30}$ \\
$c_{11}$ & $-394260 . \mathrm{meV} \cdot \AA^{33}$ & $c_{12}$ & $134763 . \mathrm{meV} \cdot \AA^{36}$ \\
$d_{1}$ & $0.0047131 \mathrm{meV}$ & $d_{2}$ & $4.41305 \AA^{3}$ \\
\hline \hline
\end{tabular}

dissipation of excited substrates. To describe the electronic contribution to the dissipation of translational energy of an atom in a metal, the damping rate can be directly related to the friction coefficient [158] used in the Langevin equations via the stopping power of atoms [30, 156]. In the low velocity regime, meaning, low velocity in comparison to the velocity of the electrons, this stopping power (or the related damping rate) can be calculated from the phase shifts at the Fermi energy for scattering electrons from spherical potentials. These potentials can be calculated with DFT, assuming them to be static (i.e. not dependent on electron fluctuations) and used to calculate the energy loss of atoms in homogeneous electron gasses [155, 156]. Head-Gordon and Tully [16] provided the explicit molecular-orbital expression for the electronic friction and extended the LDFA approach for a friction coefficient to outside the Markov limit, leading to a friction coefficient with memory.

The Alducin group [31] started using the LDFA for the description of $\mathrm{N}_{2}$ on $\mathrm{W}(110)$ and $\mathrm{H}_{2}$ on $\mathrm{Cu}(110)$ and since then used it to describe $\mathrm{N}_{2}$ from $\mathrm{W}(110)$ and $\mathrm{N}$ from $\mathrm{Ag}(111)$ [159]. Recently [19], they implemented nonadiabatic energy loss by including a friction force calculated with LDFA into AIMD calculations (AIMDEF). To determine the friction coefficient in this approach, they take the electron density of the undistorted surface. With it, they described hot $\mathrm{H}$ atom energy loss on $\mathrm{Pd}(100)$ and find large contributions to the energy loss from electron hole pair excitations. Furthermore, they described the life-time of $\mathrm{H}$ on $\mathrm{Pb}$ films [20] and have now extended their theory to include the interaction with the electron density of the moving atoms [160].

The Kroes group [3, 4] combined LDFA with AIMD in a post-facto fashion (AIMDEFp) to calculate the energy loss of $\mathrm{H}$ scattering from $\mathrm{Au}(111)$. That is, the energy loss to electron hole pairs was calculated after the AIMD trajectories had already been calculated. For this, they used the positions $\mathbf{r}(t)$ and velocities $\mathbf{v}(t)$ that had been recorded during the trajectory and a 
position dependent friction coefficient $\eta(\mathbf{r}(t))$ :

$$
\Delta E_{\mathrm{na}}=\int_{t=0}^{t_{\mathrm{end}}} \eta(\mathbf{r}(t)) v(t)^{2} d t
$$

They obtained the electron density from a metal slab with the atoms fixed at their equilibrium positions and calculated the friction coefficient according to Juaristi et al. [31. Using this postfacto approach, they expect the energy losses to ehp to be by $\sim 20 \%$ larger than the energy loss obtained if the Langevin equations using LDFA are applied during an AIMD trajectory. They observed for $\mathrm{H}$ atoms scattering from $\mathrm{Au}$ that the nonadiabatic average energy loss to electron hole pairs is the dominant energy dissipation channel and found that the nonadiabatic energy loss they calculated appears to be consistent with the calculations Trial et al. [161] for $\mathrm{H}$ and D on $\mathrm{Cu}(111)$ who used time-dependent DFT to obtain the friction coefficient instead of a friction coefficient obtained from static DFT.

Rittmeyer at al. [162] have suggested an improvement of LDFA for molecules that allows the treatment of the molecule including intramolecular contributions to embedding density that are accounted for by a charge partitioning scheme.

LDFA has been used to study multiple other system. Vibrational damping of adsorbate modes on metal surface can be reasonably well described using LDFA [162] and has been used to describe e.g. $\mathrm{H}_{2}$ and $\mathrm{D}_{2}$ on $\mathrm{Ru}(0001)$ [163, 164], dissipative dynamics of $\mathrm{H}$ on Pb-films [20] and vibrational quenching in associative adsorption of $\mathrm{N}_{2}$ from $\mathrm{Ru}(0001)$ [165]. Additionally, LDFA has been used to describe nonadiabatic effects on atoms and molecules at surfaces e.g. $\mathrm{H}$ in and on $\mathrm{Ni}$ [17, 166], Eley-Rideal reactions for $\mathrm{H}_{2}$ and $\mathrm{N}_{2}$ on $\mathrm{W}$ or the diffusion of $\mathrm{H}$ on $\mathrm{Ni}$ [167] and $\mathrm{H}$ in $\mathrm{Pd}$ [30, 74], also in good agreement with experimental observations.

The electronic friction approach has proven useful to describe processes in the weakly nonadiabatic limit such as vibrational relaxations, inelastic molecule surface scattering or hotelectron mediated femtosecond laser induced reactions [163] as well as vibrational damping of adsorbate modes on metal surfaces [162] and hot-atom mediated femtochemistry [153]. But in the usage of LDFA described above, the friction coefficient is assumed to be isotropic (and therefore derivable from a homogeneous electron gas). This assumption is taken because it simplifies calculations not only regarding the shape of the tensor, but also, because the calculation for a phase-shift for anything else but a homogeneous electron gas would require to replace the approximation of a symmetric spherical potential by a more complicated potential which would not allow to describe the phase shift as simply as presented by Echenique et al. [155]. An alternative would be the calculation of the friction coefficient from the transition probability, but this would require the calculation of electronic excitation energies between occupied and unoccupied orbitals which cannot be done using theories such as EAM or EMT. Given the high dimensional shape for the friction tensor, it is very cost-intensive to calculate the tensor and in the past, if the friction coefficient was treated as a tensor, it has usually only been calculated for reduced 
dimensions as e.g. for the description of $\mathrm{CO}$ on metal surfaces [168-171] $\mathrm{CN}$ or $\mathrm{N}_{2}$ on $\mathrm{Pt}(111)$ or $\mathrm{Ru}(0001)$ [168, 172]. Calculating the position dependence of the friction coefficient can be done with time-dependent DFT [173] as has been done for $\mathrm{H}$ and $\mathrm{D}$ on $\mathrm{Cu}(111)$ [161, 173, 174]. To account for quantum mechanical effects, a wave package approach can be used or open-system density matrix theory 153$]$.

The implementation of LDFA with the homogeneous electron gas approximation into the EMT has perhaps an advantage over methods such as AIMD, because EMT already provides the background electron density, that is, not only the electron density of the surface atoms but also the perturbation in that electron density due to the presence of the $\mathrm{H}$ atom.

\subsection{The $\mathrm{H} / \mathrm{Au}$-system}

\section{Bulk and Surface Properties}

The primitive unit cell is the smallest building block from which a crystal lattice can be built by translational displacement. To classify crystals, a unit cell is chosen that is slightly larger and chosen such that its sides have the shortest lengths and the angles of its vectors are as close to perpendicular as possible. The lengths of the sides in three dimensions are denotes as $a, b$ and $c$, its three lattice vectors as $\mathbf{a}, \mathbf{b}$ and $\mathbf{c}$ and the angles between the vectors as $\alpha, \beta$ and $\gamma$. Depending on the length of the sides and the angle between them, fourteen distinct space lattices can be identified in three dimensions, the so-called Bravais lattices. A cut through a crystal gives rise to a surface with a structure depending on the direction of the cut. This surface is defined by the crystal plane along which the cut happens and described by the Miller indices $h, k$ and $l$, as are directions on the surface. Every plane will intersect $a, b$ or $c$ at a certain distance. If the reciprocal of the smallest intersection distance is taken and multiplied with such a factor that leads to three integers, the Miller indices have been obtained. Negative indices are denoted by a bar. If only the a lattice vector would be cut by a plane, a (100) plane would be obtained, if all are cut, a (111) [175]. Directions on the surface are denoted in the same manner by the Miller indices, only replacing the parentheses with brackets to distinguish direction from plane.

For the description of e.g. electronic band structure and phonons the first Brillouin zone is important. It can be built by connecting an origin reciprocal lattice point with each of its neighboring reciprocal lattice points. At the midpoint of the connecting lines, perpendicular lines are drawn and the smallest area that contains the origin and is enclosed by the lines is the Brillouin zone. The center of the Brillouin zone is denoted by $\Gamma$ and its equivalent in real space is the Wigner-Seitz cell. The reciprocal lattice is related to the real space via the basis vectors 
of the real space [176]:

$$
\mathbf{a}^{*} \equiv \frac{2 \pi}{V}(\mathbf{b} \times \mathbf{c}), \quad \mathbf{b}^{*} \equiv \frac{2 \pi}{V}(\mathbf{c} \times \mathbf{a}), \quad \mathbf{c}^{*} \equiv \frac{2 \pi}{V}(\mathbf{a} \times \mathbf{b})
$$

\subsubsection{FCC-metals and Gold}

Gold has a face-centered cubic $(f c c)$ unit cell and a lattice constant of $4.08 \AA[177]$. An $f c c$ crystal has ABC-stacking which means that the atoms of the first layer are stacked below every second of the hollow sites of the first layer and those of the third layer below the other half of the hollow sites, so that only in every third layer the structure repeats itself (see Fig.2.1(a)). On an $f c c(111)$-surface, this leads to two types of hollow sites; one, the hexagonal close-packed $(h c p)$-hollow site, where an atom is directly in the layer below and another, the face-centered cubic $f c c$-hollow site, where the next $\mathrm{Au}$ atom is to be found two layers below. Further distinct surface sites on the $\mathrm{Au}(111)$-surface are the top site, marking the top of a $\mathrm{Au}$ atom and the bridge-site, the point where two neighboring atoms meet in the surface layer. Below the surface, there are also two distinct sites available. One is located directly below a Au atom: it would be e.g. in the first subsurface layer, directly under a surface atom. It is the tetrahedral under top (TUT) site. The second is located one layer further away, that is, the hollow formed in e.g. the second subsurface layer below the top atom due to the ABC-stacking. This hollow site is called octahedral site (oct.).

\section{Au surface structure}

DFT-GGA-functionals are known to overestimate the lattice constant, especially for heavier elements [178]. RPBE and the SRP-functional give the highest value with $a_{0} \approx 4.22 \AA$ [178] and $a_{0}=4.20$ [4], PW91 [41, 179] and PBE [43, 180] both range around $a_{0}=4.18 \AA$. Several

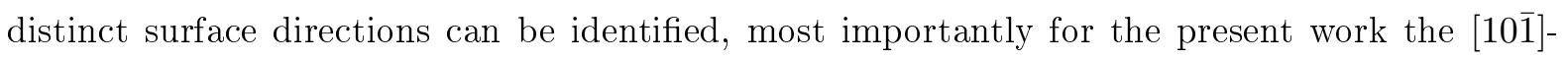

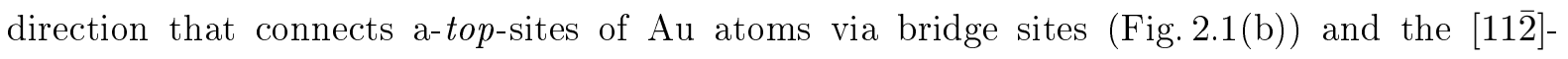

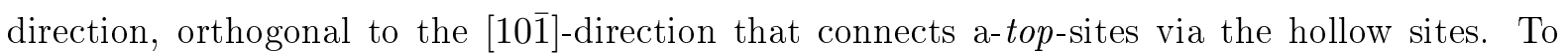
describe the incidence $\mathrm{H}$-atom beam and for scattering, I define two angles: the polar angle $\theta$ is the angle to the surface normal. The azimuth angle $\phi$ is the angle with the [110]-direction where $\phi=0^{\circ}$ corresponds to the [11 0$]$-direction. The $\mathrm{Au}(111)$-surface reconstructs to form domains of $\sqrt{3} \times 22[32$, 33] $(\sqrt{3} \times 23[34,35])$ reconstructions along the [110]-direction (discommensuration lines). This means that two-and-twenty (23) Au atoms contract in such a manner that on the space normally inhabited by these atoms, 23 (24) Au atoms are placed. Fig.2.2 gives an impression of how the surface looks and the relation of the surface directions to it. The discommensuration lines in turn form the secondary herringbone structure that can be observed on wide terraces and has a periodicity of about $30 \mathrm{~nm}[181$. 

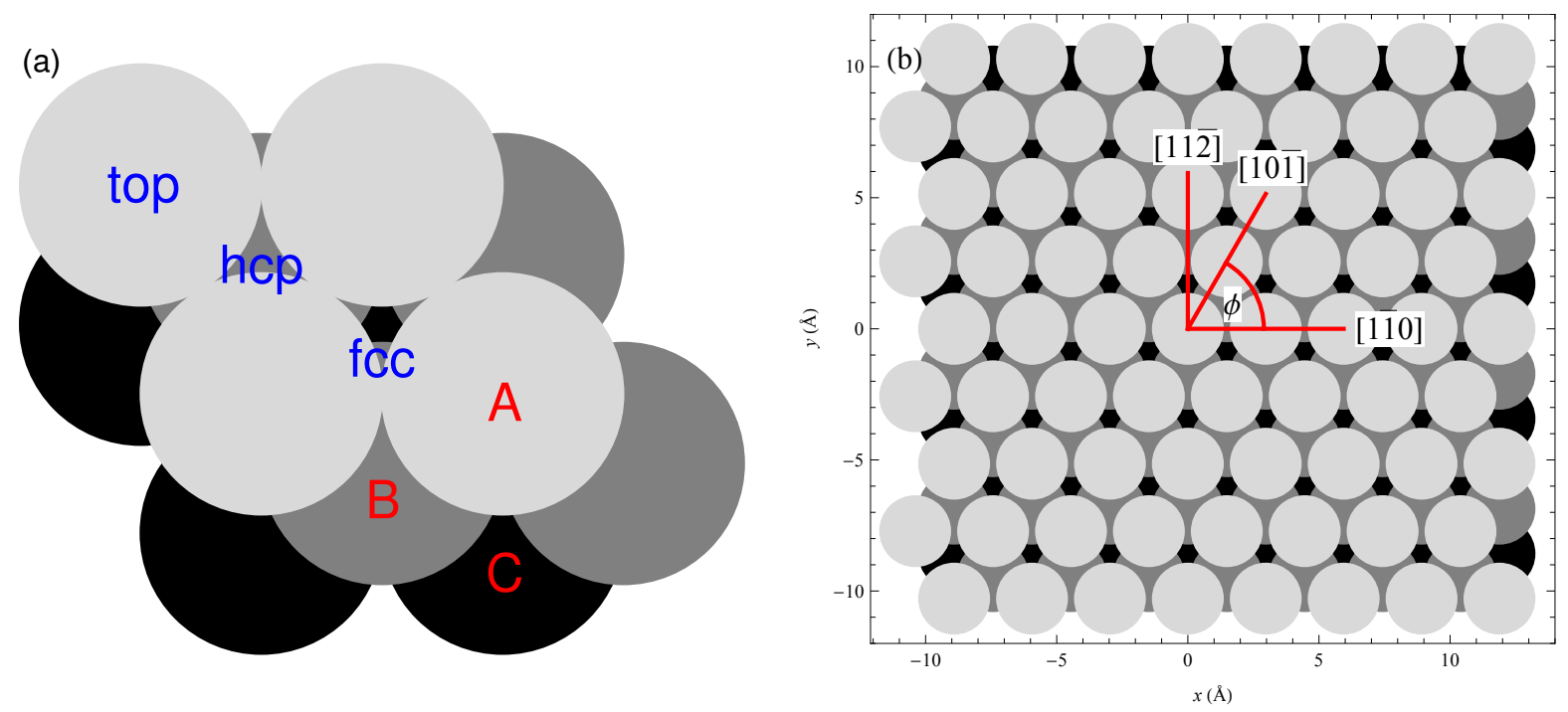

Figure 2.1.: (a) Surface sites and stacking of an $f c c$-surface. Au atoms in the surface layer (A) are indicated in light grey, in the first subsurface layer (B) in dark grey and in the second subsurface layer in black (C). (b) shows a larger view of the (111)-surface with its $C_{3^{-}}$ symmetry, two surface directions and the azimuth angle $\phi$.

Surface reconstruction is driven by excess surface stress, meaning that the atoms at the surface have, compared to the bulk atoms, unsatisfied dangling bonds. This tensile stress can be satisfied by moving the atoms closer together as happens in the reconstruction. Although why some $f c c$ (111) surfaces reconstruct and others do not is still a matter of discussion, much as by which energetic mechanisms the reconstruction is lifted by adding other atoms to the surface [180]. While in theoretical simulations, only the $\sqrt{3} \times 22 / 23$ supercell itself appears to have been
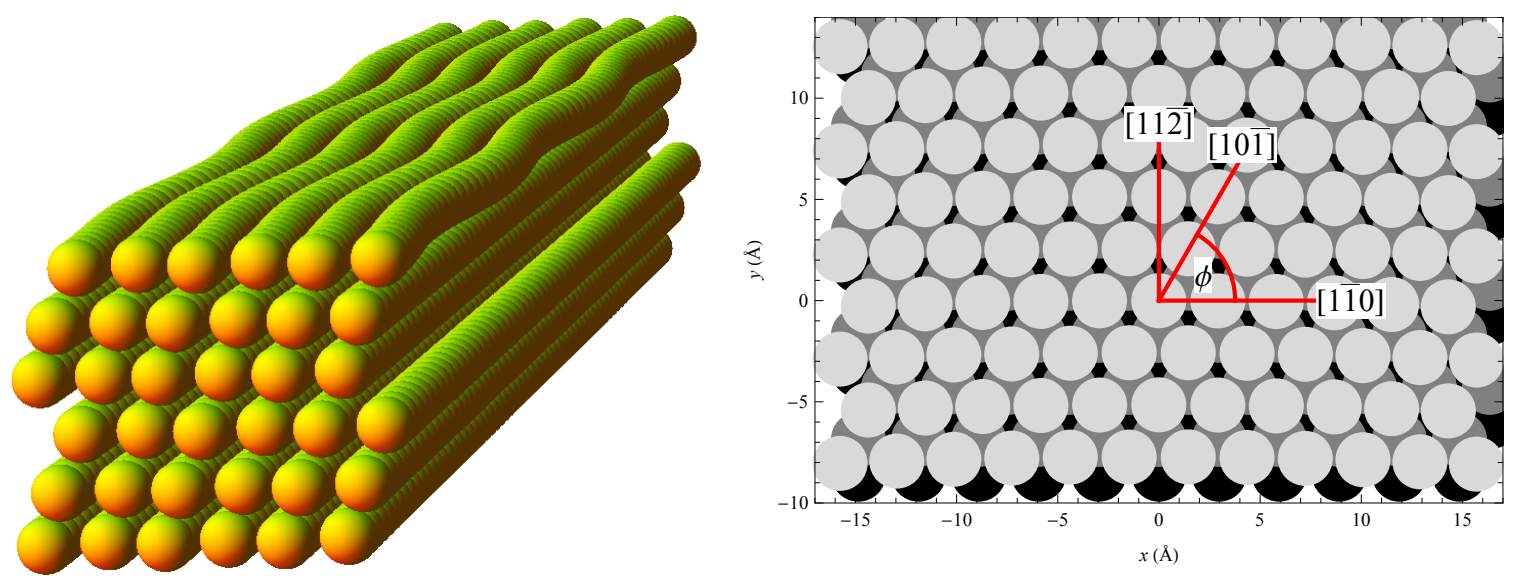

Figure 2.2.: The discommensuration lines of the reconstructed $\mathrm{Au}(111)$-surface is shown from a sideview of a six-layered slab where the discommensuration lines show as waves in the surface layer (left) and from an on-top view including the surface directions and angle $\phi$ (right). 
studied (and thus not the entire herringbone), $\mathrm{Au}(111)$ reconstruction is not solely made of the secondary herringbone patterns, but shows elbow structures [43] or u-structures [32]. Repain et

al. [181] found that the $\sqrt{3} \times 22$ reconstruction structure is closely associated with step structure where the direction of the step dictates the direction of the discommensuration lines. The reconstruction pattern is stable up to $\sim 865 \mathrm{~K}$ and at higher temperatures a partially disordered phase with hexagonal symmetry forms [182]. Furthermore, Hoss et al. [183] observed surface premelting: the surface itself is observed to lose its ordered structure entirely at $1070 \mathrm{~K}$, creating a thin quasi-liquid layer. They furthermore observe the lifting of reconstruction at $950 \pm 50 \mathrm{~K}$.

The formation of the reconstruction has been attempted to be explained by one- and twodimensional Frenkel-Kontorova models [179, 184-186] or attempts made to reproduce the stable discommensuration lines with embedded atom like methods [187, 188], the latter with little success. One of the problems in simulation is the large supercell needed to describe surface reconstruction which makes $a b$ initio simulations using DFT almost forbiddingly expensive. There are only few studies in DFT for the discommensuration lines using various functionals (PBE [43], PBE with van-der-Waals corrections [43], PW91 [41, 42]), all of which predict the discommensuration lines to be stable and can reproduce the experimentally observed structure. Furthermore, they show that the reconstruction influences adsorption behavior [43]. Torres et al. [42] investigated discommensuration of different periodicity $\sqrt{3} \times p 2$ and found $p=22$ and $p=23$ to be the most stable pattern, again more stable than the unreconstructed surface. Recently, the electronic structure [189] and reconstruction at stepped surfaces in a mixture of DFT and EAM-type calculations [190] have been studied. Additionally, tight-binding simulations have been done with large supercells ( $\sim 50000$ atoms) to investigate the structure of the herringbone reconstruction, giving the correct ratio of 22 to 23 surface atoms, reproducing the correct geometrical behavior during MD simulations but proving to be only a local and not a global minimum [191].

\subsubsection{Hydrogen in Interaction with Metals}

While the field itself is active, hydrogen atoms in interaction with Au have so far seen little investigation. The study of $\mathrm{H}$ atoms on metals has mostly focused on determining the extend of adsorption site, vibrational frequencies of the adsorbate and electron hole excitation and diffusion.

Dissociative adsorption of $\mathrm{H}_{2}$ on $\mathrm{Au}$ is strongly activated. The adsorption of $\mathrm{H}$ on $\mathrm{Au}$ films was studied using temperature programmed desorption (TPD) [192-195] and the activation energy of desorption of $0.5-0.6 \mathrm{eV}$ and the sticking probability at $78 \mathrm{~K}$ has been determined. Experimentally, considerable evidence for the interaction with electron hole pairs has been observed for $\mathrm{H}$ in interaction with coinage metals. For example, chemicurrents were measured when a polycrystalline Au covered metal-insolator-metal structure was exposed to a hydrogen atom flux, and were caused by a Langmuir-Hinshelwood recombination reaction. Here, a clear 
isotope effect was observed, as well as subsurface hydrogen species [58, 59, 196]. Further reaction induced chemicurrents were observed using $\mathrm{Ag} / p$-Si Schottky diodes for $\mathrm{H}$ and $\mathrm{D}$ chemisorption on $\mathrm{Ag}$ and on $\mathrm{Cu}(111)$ [197-200]. They are in good agreement with calculations of chemicurrents for $\mathrm{H}$ impacting on $\mathrm{Cu}(111)$ using electronic friction and make predictions for $\mathrm{H}$ impacting on $\operatorname{Ag}(111)$ [201]. Kovacs et al. 202 measured the emission current occasioned by kinetically induced electron emission in the collision of $\mathrm{H}$ and $\mathrm{D}$ atoms with $\mathrm{Au}, \mathrm{Cu}$ and $\mathrm{Ag}$ films. Parallel vibrations of $\mathrm{H}$ and $\mathrm{D}$ on $\mathrm{Cu}(111)$ were detected by infrared reflection spectroscopy and found that the atoms absorb in the threefold hollow sites and the life-time of the vibration explained by electron hole pair damping [203]. Ishikawa et al. [67] measured the diffusion of $\mathrm{H}$ in $\mathrm{Au}$ around room temperature and found that it obeys the Arrhenius law also considering the data measured by Eichenauer et al. at 800-1200 K [204].

The description of $\mathrm{H}$ in interaction with jellium hosts was already an active field of study forty years ago 205]. Later, using EMT, Nørskov et al. described $\mathrm{H}$ in interaction with metal systems to obtain qualitative information about chemisorption energies, interstitial hydrogen, hydrogen diffusion and hydrogen molecule formation [206].

Ferrin et al. [207] considered the adsorption of $\mathrm{H}$ to metals with PW91 and found that $\mathrm{H}$ on $\mathrm{Au}(111)$ is most stably adsorbed to the surface $(-2.18 \mathrm{eV})$ in the $f c c$ - and $h c p$-surface site. Absorption into the bulk is less favorable within its preferred TUT $(-1.45--1.55 \mathrm{eV})$ absorption site. These findings are in agreement with the simulations of Greeley et al. [39] who also found a (slight) preference for the $f c c$-adsorption site $(-2.22 \mathrm{eV}$ (PW91) and $-2.07 \mathrm{eV}$ (RPBE) and for the TUT subsurface site $(-1.47 \mathrm{eV}$ (PW91) and $-1.36 \mathrm{eV}(\mathrm{RPBE}))$. The gas-phase $\mathrm{H}_{2}$ molecule is energetically more favorable than an adsorbed $\mathrm{H}$ at the $\mathrm{Au}(111)$ surface. They also studied diffusion pathways of the H-atoms. In my master thesis [208], using the SRP-functional, I found a similar preference for surface adsorption at the $f c c$ - and $h c p$-sites. Kroes and coworkers [3, 4] studied the scattering of $\mathrm{H}$ from $\mathrm{Au}(111)$ electronically adiabatically using AIMD and nonadiabatically using AIMDEFp.

A large field of study is $\mathrm{H}$ in interaction with $\mathrm{Cu}$. Strömquist et al. [23] considered electronically adiabatic $\mathrm{H}$ on $\mathrm{Cu}(111)$ in detail by means of fitting $a b$ initio energies with the EMT and suggested that the energy dissipation to ehp could make out a large pathway in the overall energy loss. They furthermore calculated sticking probabilities, finding that with a slab relaxed to the presence of the $\mathrm{H}$ atom, absorption is not activated, that energy loss to phonons is ineffective and that the corrugation of the surface is important for scattering results. For the same system, the three-fold hollow adsorption site has been determined using DFT with GGA-functionals [39, 207, 209-212], LDA [213, 214] and EMT [23, 213]. Experimentally, the adsorption site has been suggested to be the threefold hollow site [203, 215], the bridge site [216] (although later reinterpreted to threefold hollow [203, 213, 215]) and preferential subsurface absorption [217, 218]. Subsurface absorption has been studied in a number of DFT 
simulations [39, 207, 211] and was always found to be less favorable than surface adsorption. Experimentally, it was also suggested to be coverage [215] and temperature [219] dependent. The coverage dependence could not be reproduced theoretically [211, 220]. Barriers for diffusion were also considered in multiple theoretical calculations [207, 209, 211, 220].

Shalashilin et al. considered hot atom motion of $\mathrm{H}$ on $\mathrm{Cu}(111)$ using a static surface PES with dissipation to phonons mimicked by a friction and a stochastic force 221].

Further recent studies of $\mathrm{H}$ in interaction with surface involve $\mathrm{H}$ scattering from graphene [222] and hot $\mathrm{H}$ atoms on $\operatorname{Pd}(100)$ [19, 160] as well as $\mathrm{H}$ vibrations on $\mathrm{Pb}(111)$ [20].

\subsection{Fitting Procedure}

The following section has to a large extend already been published [21, 22].

\subsubsection{The DFT ab initio input data set}

To construct a potential energy surface with analytic form, in the first step, an input data set needs to be assembled that samples the configuration space. For the EMT fit, the input data consisted of two data sets: the first one sampled the dependence of the energy on the positions of the surface atoms. This one consisted of 200 points taken from a non-penetrating doublebounce AIMD trajectory of an $\mathrm{H}$-atom colliding with $5 \mathrm{eV}$ with a $\mathrm{Au}(111)$ surface thermalized to a temperature of $120 \mathrm{~K}$ (provided by Geert-Jan Kroes [3, 4]).

The second data set used a grid in configuration space with $520 \mathrm{H}$ atom positions. Here, $\mathrm{Au}$ atoms were fixed to the coordinates of the relaxed $f c c$ surface. This grid probed ten symmetry sites on the $\mathrm{Au}(111)$ surface (Fig. 2.3 in steps of $0.2 \AA$ above the surface to $5 \AA$ below the surface. The symmetry sites include the top-site (1) and the two hollow sites on the surface, $h c p(7)$ and fcc hollow site (10, see Fig.2.3 and Tab.2.2.

The $a b$ initio energies for the individual H-atom positions I obtained with the Vienna $a b$ initio simulation (VASP) 5.2 package package [223-226]. To match the AIMD calculations, I used ultrasoft pseudo potentials and the SRP48-GGA functional suggested by Díaz et al. [5] (see section 2.3 in adaption for VASP [12]. I had already calculated a data set for a $3 \times 3 \times 4$ super cell during my master thesis [208, but recalculated the data set using a $2 \times 2 \times 4$ super cell to match the $2 \times 2 \times 4$ cell of the AIMD. For this, I used $20 \times 20 \times 1$ gamma-centered k-pointmesh, a Fermi level smearing of $0.1 \mathrm{eV}$ and an energy cut-off of $300 \mathrm{eV}$ (see attachment) and did spin-polarized calculation. As a reference energy, which I subtracted for all $a b$ initio points, I took the energy of the undisturbed system of the gold surface with all Au atoms in their relaxed positions and an $\mathrm{H}$ atom $6 \AA$ above this surface where it does not interact with the surface. All potential energy values given subsequently are given relative to the reference energy. Further 


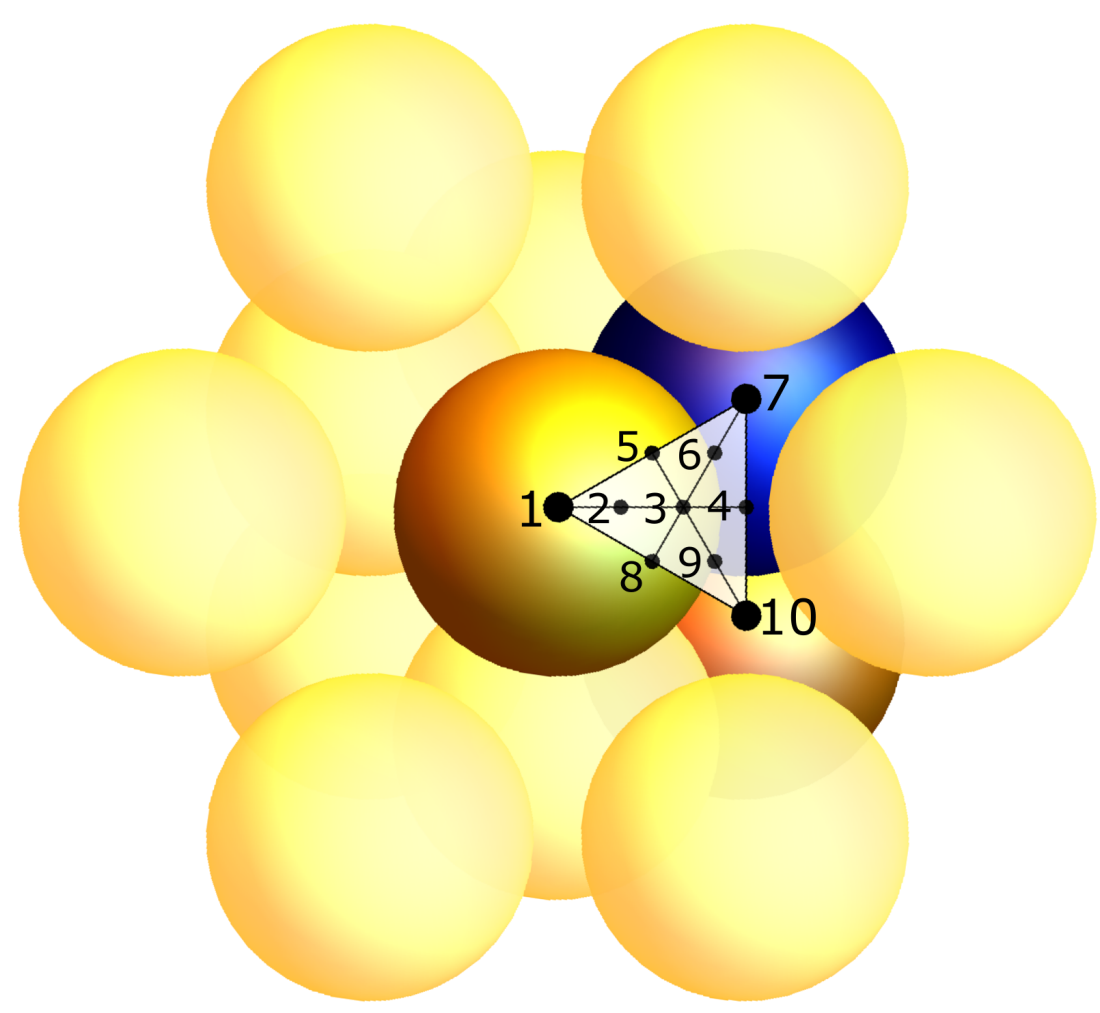

Figure 2.3.: The sampling sites for the generation of the input data set to the EMT fit. The yellow spheres represent the positions of the $\mathrm{Au}$ atoms for the first three layers of the $\mathrm{Au}(111)$ surface. The blue sphere marks the Au atom below an hcp and the orange atom below an fcc-site. The black points mark the ten sampling sites.

Table 2.2.: The $x$ and $y$ coordinates of the sampling sites and their names. Numbers according to Fig. 2.3 $a=\frac{a_{\circ}}{\sqrt{2}}$ with $a_{0}$ the lattice constant and $a$ the nearest-neighbor distance.

* Sites named according to [209].

** Self administered names and table reproduced from [208].

\begin{tabular}{cccll}
\hline \hline Site & $x$ coordinate & $y$ coordinate & Abbreviation & Site \\
\hline 1 & 0 & 0 & top & top site \\
2 & $\frac{a}{6}$ & 0 & tso $^{* *}$ & between ott and top site \\
3 & $\frac{a}{3}$ & 0 & ott $^{* *}$ & one third between top sites \\
4 & $\frac{a}{2}$ & 0 & bri $^{* *}$ & bridge site \\
5 & $\frac{a}{4}$ & $\frac{\sqrt{3}}{12} a$ & $\mathrm{fht}^{*}$ & between hcp hollow and top \\
6 & $\frac{5}{12} a$ & $\frac{\sqrt{3}}{12} a$ & hho $^{* *}$ & between ott and hcp hollow site \\
7 & $\frac{a}{2}$ & $\frac{\sqrt{3}}{6} a$ & hcp $^{*}$ & hexagonal cubic package hollow \\
8 & $\frac{a}{4}$ & $-\frac{\sqrt{3}}{12} a$ & hht $^{*}$ & between fcc hollow and top \\
9 & $\frac{5}{12} a$ & $-\frac{\sqrt{3}}{12} a$ & $\mathrm{fho}^{* *}$ & between fcc hollow and ott \\
10 & $\frac{a}{2}$ & $-\frac{\sqrt{3}}{6} a$ & $\mathrm{fcc}$ & face centred cubic hollow \\
\hline \hline
\end{tabular}


details on the calculations are specified in the attachment to this thesis, including the input files for VASP.

For the fit, I excluded all configuration/energy pairs that had a higher potential energy than $20 \mathrm{eV}$, reducing the entire data set from 760 constituents to 720 constituents.

\subsubsection{The Fit}

For the fit, I used the MD_tian program, a package that has been written in the course of the last three years in our group, written by myself, Dr. Alexander Kandratsenka and Prof. Dr. Daniel Auerbach (see also attachment $\mathrm{B}$ ). It contains the fitting procedure and MD-simulation procedure, including an implementation of the analytic equations for the EMT forces. To determine the values of the 14 EMT parameters, I used a Levenberg-Marquardt [227, 228] damped least squares procedure which minimizes the rms deviation of the energy values given by DFT and the EMT PES. Asides from the input data derived from ab initio DFT, I used two further information for the selection of an optimal fit: the values of bulk-properties of gold and the H-Au binding energy (see Tab. 3.3). Disregarding these constrains results in parameters that do not give reasonable values for the bulk properties of $\mathrm{Au}$ and the $\mathrm{H}-\mathrm{Au}$ binding energy. To defeat this difficulty, I constrained some of the parameters. For $\mathrm{Au}$, I fixed $\varepsilon_{0}$ to reproduce the cohesive energy, $s_{0}$ to reproduce the lattice constant obtained from DFT, and $\lambda$ to give the correct bulk modulus. For hydrogen, I fixed $\varepsilon_{0}$ and $s_{0}$ to the values recommended by Strömquist [23]. Additionally, I ensured that the EMT-based PES would reproduce the experimentally known $\mathrm{H}-\mathrm{Au}$ gasphase diatomic bond energy [229] and confirmed that the Au slab was stable to up to 1000 K. For the fit, I used the parameters suggested by Jacobsen [2] and Strömquist [23] as initial guesses.

\subsection{MD simulations}

I performed adiabatic and nonadiabatic MD simulations for $\mathrm{H}$ in interaction with $\mathrm{Au}(111)$ with propagation times of $120 \mathrm{fs}$ or $1 \mathrm{ps}$ and a time step of $0.1 \mathrm{fs}$ using the MD_tian program. This package has been developed over the last years in our research group and was written by Dr. Alexander Kandratsenka, Prof. Dr. Daniel J. Auerbach and myself. It encompasses the fitting procedure and MD-simulation procedure, including an implementation of the analytic expressions for the EMT forces (see attachment B).

The Au slab was equilibrated over 5 ps by assigning velocities from the Maxwell-Boltzmann distribution corresponding to a given temperature to the Au atoms. After that, the simulation was continued for another $100 \mathrm{ps}$ and the Au atom configurations and velocities sampled every $100 \mathrm{fs}$. Each trajectory randomly assumes one of these 1000 configurations and velocities at the start of the trajectory to make sure that I sampled a wide variety of Au configurations for 
each temperature. For a $6 \times 6 \times 6$ slab the atoms of the lowest three levels were fixed to their ideal lattice positions to simulate the bulk structure and keep the slab stationary during the simulations, and for a $6 \times 6 \times 4$ slab only the atoms of the lowest layers were fixed to their ideal lattice positions.

For MD simulations of $\mathrm{H}$ in interaction with $\mathrm{Au}(111)$, I have classified the outcomes into four classes: (1) scattered if the $\mathrm{H}$ atom at the end of the propagation time is further away than $5 \AA$ from the surface, (2) adsorbed if the $\mathrm{H}$ atom is above the first layer of Au atoms of the slab but closer than $3 \AA$, (3) absorbed if the $\mathrm{H}$ atom remains within the slab and (4) transmitted if the $\mathrm{H}$ atom has passed through the slab at the end of the propagation time, that is, finds itself below the $\mathrm{Au}$ atoms of the bottom layer of the slab. $\mathrm{H}$ atoms belonging to the last case are estimated to continue deeper into the bulk and to make no contribution to the scattered $\mathrm{H}$ atoms. If the $\mathrm{H}$ atom finds itself inside the slab, I class its position according to the layer it finds itself in. For this, I use the perfect $f c c$ structure. In the perfect $f c c$-structure, the Au atoms of the surface are centered at $z=0 \AA$; any $\mathrm{H}$ atom that finds itself above $z=0 \AA$ (and below $3 \AA$ ) I consider as being on the surface. Likewise, any $\mathrm{H}$ atom that finds itself between $z=0 \AA$ and the atoms of the first subsurface layer centered at $z=-a_{0} / \sqrt{3}$ I consider as being absorbed in the first subsurface interlayer space (sublayer), \&c. I am conscious that this division is only a rough one, especially when the Au atoms find themselves in thermal motion or the slab relaxes from its perfect $f c c$-structure. However, any other classification would be vastly more complicated. I therefore chose a very simple model to classify the position in $z$-coordinate of the $\mathrm{H}$ atoms, it being easily reproducible and readily understood.

Furthermore, I classified the trajectories of scattered $\mathrm{H}$ atoms into how many collision the $\mathrm{H}$ atoms experience with the surface. This I did by monitoring the embedding electronic density of a $\mathrm{H}$ atom in each step. If it underwent a maximum between the present and the two previous steps and an background electron density $>0.25 \AA^{-3}$, I consider the $\mathrm{H}$ atom to have experienced a bounce, for the closer an $\mathrm{H}$ atom approaches an $\mathrm{Au}$ atom, the higher the electron density it will experience, peaking at the closest point to an Au atom. I discriminate between three types of bounce events: one (single-bounce events), two (double-bounce events) or more (multibounce events) collisions with a surface atom. Of course, as any classification based on a threshold, the classification given here is not entirely accurate, but I expect it to be reasonable enough.

In each section of chapter 3.3 , I show three tables containing information about scattering and sticking probabilities as well as the peak and mean energy loss of the total and differential energy loss distributions (ELDs) belonging to different scattering conditions. For the differential energy loss distributions, I give a lesser number of significant figures to account for the fact that these distributions usually have a low signal-to-noise ratio which makes it difficult to assign the peak position. Wherever the peak position for a differential energy loss distribution at a specific scattering angle is given, it should therefore be treated with caution. Here, 'total energy 
loss distribution' is to be understood as that energy loss distribution that is obtained when the contribution of all scattering trajectories is considered. In contrast, 'differential energy loss distribution' refers to the ELD that is obtained when only trajectories are considered in which the $\mathrm{H}$ atoms leave the surface under specific scattering angles. This nomenclature has been chosen analogous to total and differential cross section. If ELD is mentioned without preceding adjective (outside chapter 44, the description applies to both differential and total energy loss distribution.

All energy loss distributions shown here are of the scattering flux. To construct them, unless otherwise noted, I took a binning interval of $10 \mathrm{meV}$. To obtain the differential energy loss distributions for different scattering angles, I selected all the trajectories where the $\mathrm{H}$ atoms scattered within $5^{\circ}$ to the vector along the selected scattering direction. By that I mean that for observing scattering for $\theta_{\text {out }}=45^{\circ}$ along the [101] -direction, I defined a vector pointing in this direction and selected all trajectories where the $\mathrm{H}$ atom had a scattering velocity vector within $5^{\circ}$ as contributing to this differential energy loss distribution. This scheme can be imagined as a disk of respective radius that is part of the spherical surface centered at the coordinate origin, thereby mimicking the hole of a detector that scans for scattering $\mathrm{H}$ atoms at a constant distance to the surface. I adopted this scheme to obtain differential energy loss distributions for individual scattering angles that were constructed in a manner that follows the experimental procedure as closely as I could devise.

For the total angular distributions, where double counting would have a serious influence on the shape of the distribution, I got the better of this drawback by employing a different selection scheme: I created a grid in $\theta_{\text {out }}$ and $\phi_{\text {out }}$, ranging from $\theta_{\text {out }}=0^{\circ}$ to $\theta_{\text {out }}=90^{\circ}$ with $\Delta \theta_{\text {out }}=10^{\circ}$ and from $\phi_{\text {out }}=0^{\circ}$ to $\phi_{\text {out }}=90^{\circ}$ with $\Delta \phi_{\text {out }}=10^{\circ}$ and binned the returning trajectories with this grid according to their exit angle. I corrected the number of counts in each interval by a factor of $1 / \sin \left(\theta_{\text {out }}\right)$ to account for the shrinking of the binning surface with shrinking polar angle.

Furthermore, I constructed angular distributions selected after their azimuth angle, that is, not overall angular distribution but such along certain surface direction. To circumvent the problem of shrinking binning intervals or double-counting, I employed the following scheme: a cross section of a sphere that corresponds to the desired azimuth angle is created. The $\mathrm{H}$ atoms that scatter within $90 \pm 3^{\circ}$ to the vector normal to that cross section are considered as contributing to the in-plane angular distribution.

The adiabatic energy loss can be estimated by means of the Baule limit which can be represented in terms of the projectile-surface mass ratio $\mu=m / M$ :

$$
\Delta E_{\mathrm{ad}}=\frac{4 \mu}{(1+\mu)^{2}} E_{\mathrm{inc}}
$$

The simulated annealing for the investigations of the surface reconstruction of $\mathrm{Au}(111)$ was 
performed using the Langevin Dynamics as a thermostat. A higher friction coefficient of $\eta \approx$ $3 \cdot 10^{-3} \mathrm{fs}^{-1}$ was assumed. This makes the annealing simulations more effective and decreases the simulation time. 



\section{Results}

Some of the results presented here have already been published prior to this date and some formulations and figures may therefore coincide [22, 44].

\subsection{DFT-Calculations}

As mentioned in section 2.7.1, the input data set for the fit consists of two contributions: a three dimensional grid where the $\mathrm{H}$ atom samples position above and within the relaxed $\mathrm{Au}(111)$

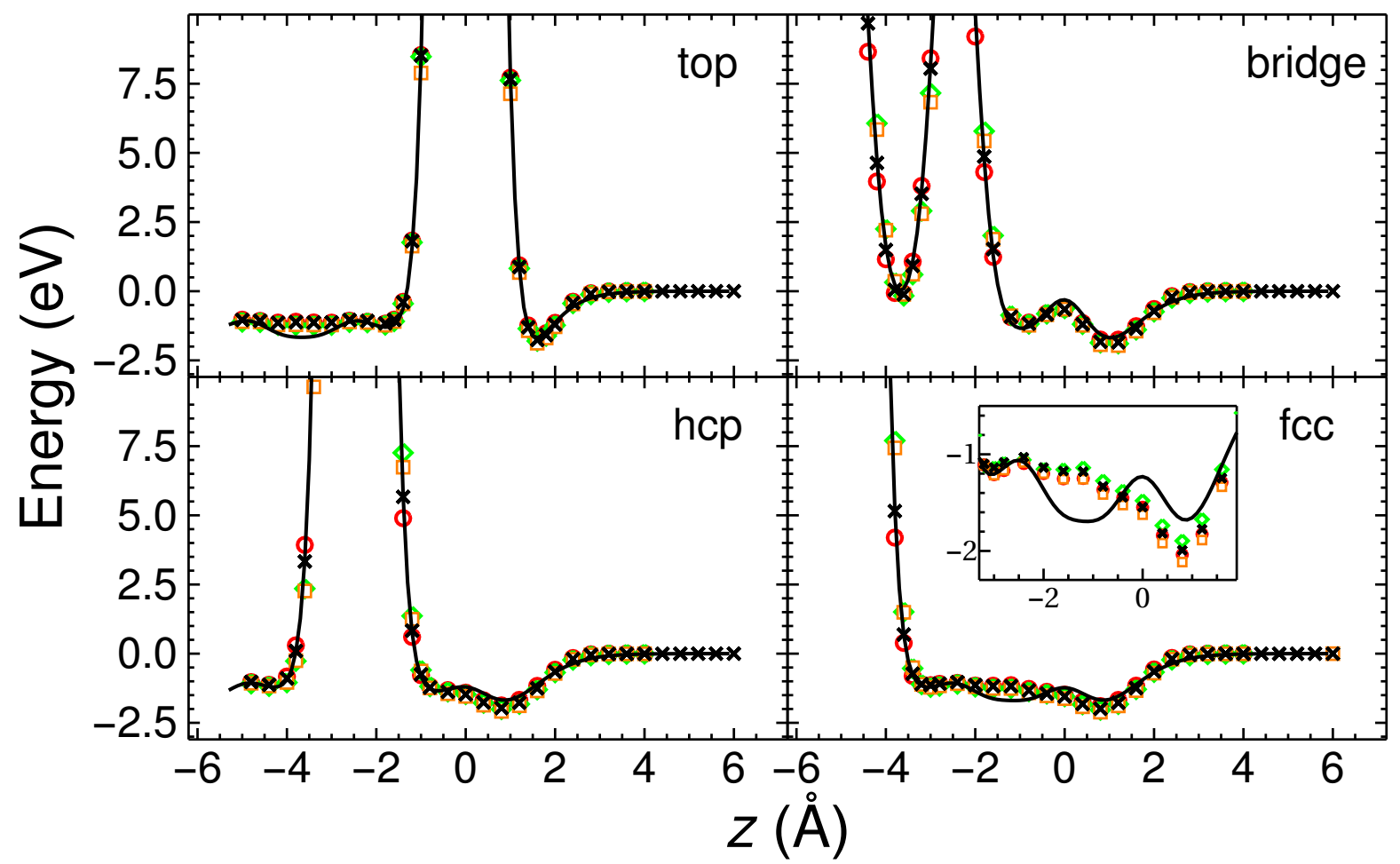

Figure 3.1.: Dependence of the potential energy on $z$-distance of the $\mathrm{H}$ atom from the surface for four symmetry sites (top, bridge, $h c p$ hollow and $f c c$ hollow). The results of ab initio calculations with ultrasoft pseudopotential (US-PP) PW91 (orange square), US-PP-PBE (red circle), US-PP-RPBE (green diamond) and US-PP-SRP48 (black cross) functionals are shown together with the EMT-fit (solid line). Figure reproduced with slight modification from [22]. 
surface and 200 points obtained from an AIMD trajectory. I had already performed calculations on a three dimensional grid (3D-grid) for a $3 \times 3 \times 4$-slab during my master thesis [208], but to be consistent with the slab used for the calculation of the AIMD-trajectories, I recalculated the energy on the three dimensional grid using a $2 \times 2 \times 4$-slab. To get an impression of how large the error in energy due to the choice of the GGA-functional is, I used the PW91, PBE and RPBE functionals with ultrasoft pseudopotentials (see Fig. 3.1) and compared the interaction of the $\mathrm{H}$ atom with a relaxed Au surface. The choice of the functional can influence the energy near local minima by up to $0.4 \mathrm{eV}$ and at the repulsive walls by up to $2 \mathrm{eV}$. The deepest minima of $-2.0 \mathrm{eV}$ are those at the two hollow sites at $0.8 \AA$ above the surface (lower panels in Fig. 3.1); the adsorption minimum at the top site is $-1.8 \mathrm{eV}$ deep and $1.6 \AA$ above the surface. At all three adsorption sites the deviation in energy values between the functionals is $0.2 \mathrm{eV}$. The depth of the adsorption wells at the hollow sites is in agreement with the energy values of -2.0 $-2.2 \mathrm{eV}$ calculated by the Mavrikakis group [39, 207], with the RPBE and PW91 functional, respectively, using the DACAPO-code. Inside the surface, the deepest absorption minima are within the tetrahedral under top (TUT) and octahedral sites of $-1.2 \mathrm{eV}$ with a difference between the functionals of $0.1 \mathrm{eV}$. While the Mavrikakis group [39, 207] predicts a preference of the TUT site and deeper absorption minima, I could not observe any marked difference in absorption energy for octahedral or TUT site.

Observe the structure the DFT calculations predict for the octahedral site (see Fig 3.1, upper left panel $z<-1.2 \AA$ ): between the Au atoms of the first and fourth layer, the potential energy landscape is almost completely flat. The deepest point of potential energy is not in the middle of the octahedral site $(z \approx-3.6 \AA)$, but slightly removed to its edges $(z \approx-3.2 \AA$ and $z \approx-4.0 \AA)$. All functionals reproduce this behavior.

VASP allows a choice between pseudopotential methods and projector augmented wave (PAW) methods for the treatment of the core electrons during the calculations [230]. In order to check how their choice affects the energy values, I compared the results of PW91 and RPBE calculations using ultrasoft pseudopotentials (US-PP) with ones using PAW [231] (Fig. 3.2). It is interesting to note that the difference in potential energy between PAW-PBE and PAW-PW91 for the adsorption sites on the surface are about half as large $(\approx 40 \mathrm{meV})$ as for US-PP-PBE and USPP-PW91 $(\approx 80 \mathrm{meV})$. The differences at the repulsive walls remain large. In addition, the flat structure observed with the ultra-soft pseudo potentials becomes less flat tending more towards a minimum in the middle of the octahedral site when the PAWs are used (see inset in Fig. 3.2. This structure is most likely due to the electronic structure of the Au atoms providing an almost uniform electron density within the octahedral site. It appears unlikely that it could be caused by the hydrogen atom being too far away from the Au atoms in the middle of the octahedral site, thereby making it (slightly) unfavorable compared to its surrounding, for then, a $\mathrm{H}$ atom in a $\mathrm{Ag}$ surface should show the same behavior, the lattice constant of $\mathrm{Ag}$ almost equaling that 


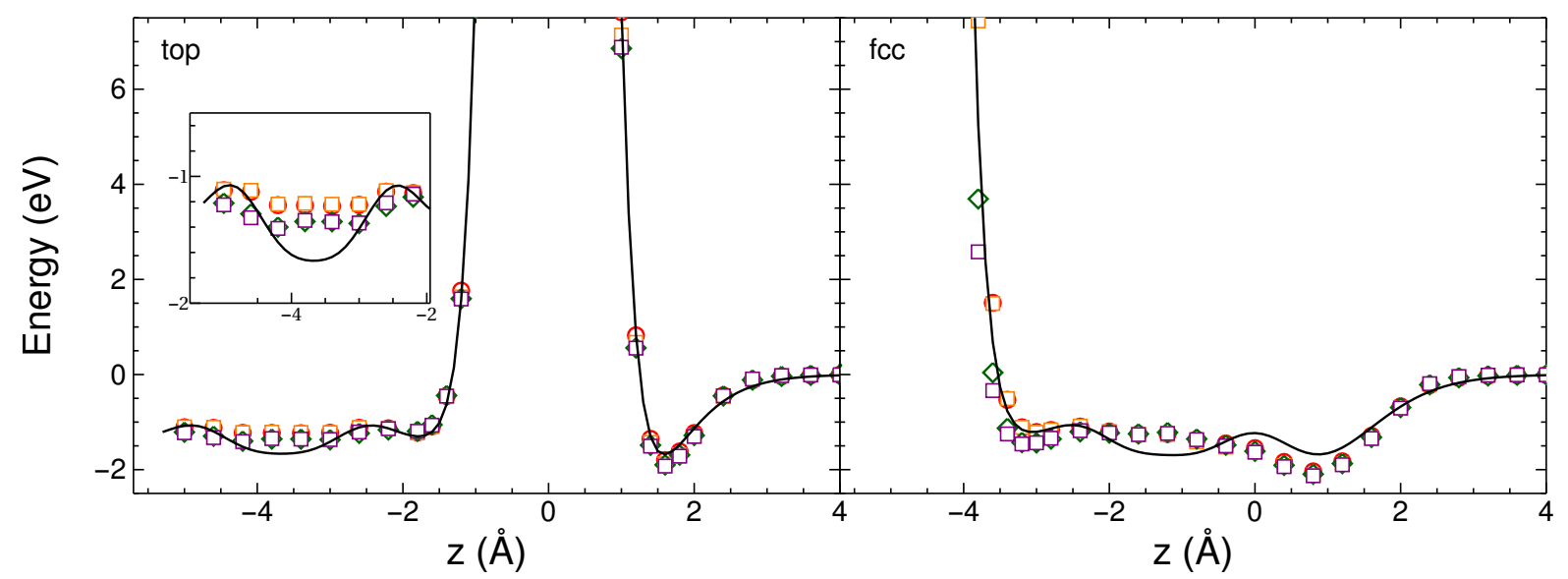

Figure 3.2.: Dependence of the potential energy on $z$-distance of the $\mathrm{H}$ atom from the surface for top site (left) and the $f c c$ hollow site (right). The inset shows an enlargement of the octahedral site. The results of $a b$ initio calculations with PAW-PW91 (purple square) [231], and PAW-PBE (dark green diamond) [231, US-PP-PBE (red circle) and US-PP-PW91 (orange squares) are compared. EMT-fit (black solid line) is also shown.

of Au. This is not the case: calculations of the octahedral sites of $\operatorname{Pd}[232$ ], $\mathrm{Ag}$ and $\mathrm{Pt}(111)$ surfaces [233] show a clear minimum structure in the middle of the octahedral sites (see Fig. 3.3.). The flat structure of the octahedral sites therefore appears to be a specific characteristic of Au.

\subsection{Fit}

Using the fitting procedure outlined in section 2.7.2, I fitted the EMT analytic function for the energy of the system to $a b$ initio energy values derived at the level of DFT-GGA. With this fit, bulk properties of $\mathrm{Au}$ and the $\mathrm{Au}-\mathrm{H}$ bond energy are also reproduced, resulting in the EMTJAWK potential energy surface 22]. EMT-JAWK is not the only PES that can result from the fitting procedure. A comparison to other PES will be given in section 3.2.2. The first two lines of Table 3.1 show the resulting EMT potential parameters. With them, the rms error to the input data set of the fit is $0.15 \mathrm{eV}$ as compared to $0.2 \mathrm{eV}$ of previous attempts of fitting with EMT-JS [23]: the energy errors associated with the choice of the DFT functional are larger than the rms fitting error of the EMT-JAWK. Fig. 3.1 shows a comparison of four representative symmetry sites that demonstrates the quality of the fit to the DFT-data calculated on the three dimensional grid. The agreement with DFT is best when the $\mathrm{H}$ atom is close to a gold atom: the repulsive walls are fitted almost perfectly. The largest deviations occur for the octahedral sites and the $f c c$ surface hollow site. In these sites, the fit deviates by up to $\approx 0.5 \mathrm{eV}$. Although the flat structure predicted by DFT (see section 3.1) for the octahedral site can be reproduced 


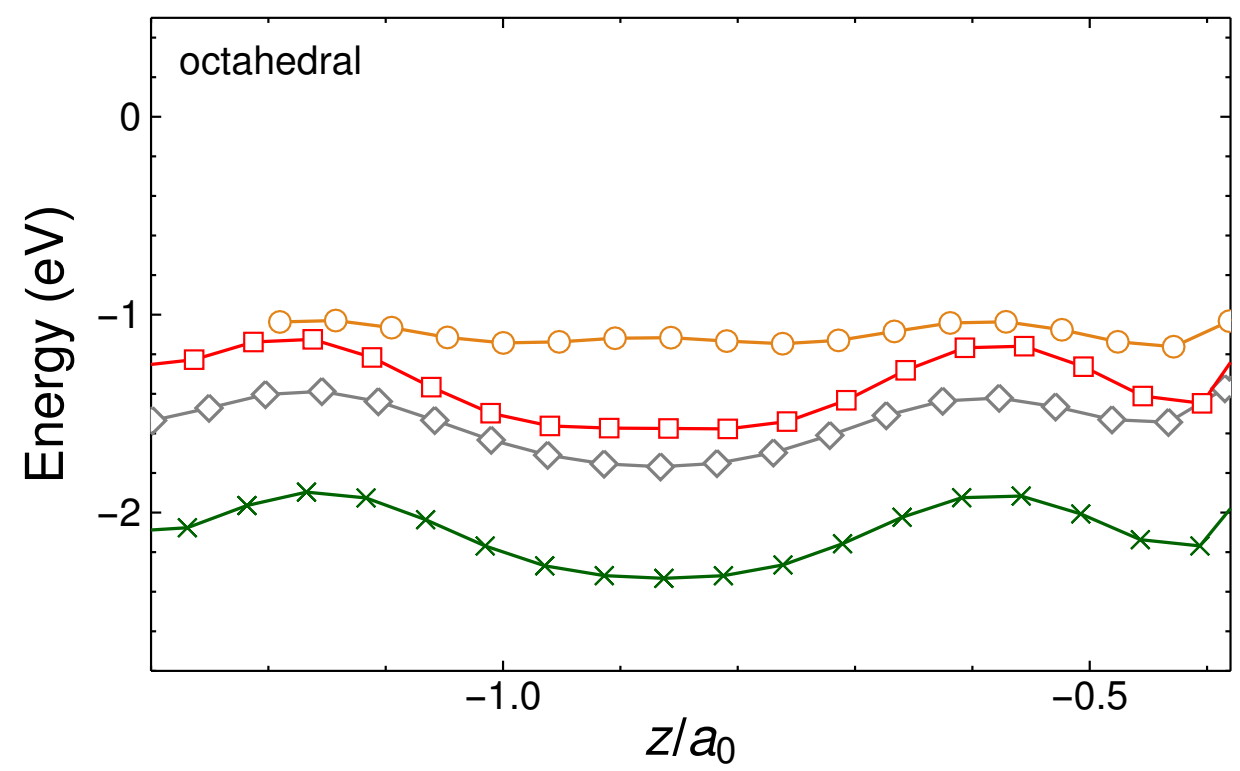

Figure 3.3.: Dependence of the potential energy on the position of the $H$ atom in the surface for the octahedral site. The results of $a b$ initio calculations for Au (US-PP SRP48-GGA, empty yellow) and $\mathrm{Pd}$ 232. (PAW-PBE, green crosses) [233], for Pt (PAW-PBE, red squares) [233] and for Ag (PAW-PBE, grey diamonds) [233] are shown. The $z$-distance is given in units of lattice constant $a_{0}$.

Table 3.1.: Parametrization for the H-Au interaction potential and electron densities of the EMT-JAWK potential of this work (see [22]) and for the EMT-JS of Strömquist [23] and Jacobsen [2].

\begin{tabular}{|c|c|c|c|c|c|c|c|}
\hline & $\eta_{2}\left(\AA^{-1}\right)$ & $n_{0}\left(\AA^{-3}\right)$ & $\varepsilon_{0}(\mathrm{eV})$ & $\lambda\left(\AA^{-1}\right)$ & $V_{0}(\mathrm{eV})$ & $\kappa\left(\AA^{-1}\right)$ & $s_{0}(\AA)$ \\
\hline \multicolumn{8}{|c|}{ EMT-JAWK } \\
\hline $\mathrm{Au}$ & 3.197 & 0.042 & $-3.8[2$ & 4.182 & 0.348 & 3.249 & 1.642 \\
\hline $\mathrm{H}$ & 4.761 & 0.203 & $-2.371[23$ & 7.549 & 0.234 & 8.747 & $0.680 \lcm{23}$ \\
\hline \multicolumn{8}{|c|}{ EMT-JS } \\
\hline $\mathrm{Au} 23]$ & 3.163 & 0.0474 & -3.8 & 4.123 & 2.321 & 5.429 & 1.588 \\
\hline $\mathrm{H}[2]$ & 5.425 & 0.182 & -2.371 & 7.727 & 0.427 & 8.863 & 0.680 \\
\hline
\end{tabular}

by some parametrization of EMT, this leads to large deviations of the bulk properties of Au from the experimental values or predicting the energy of the $\mathrm{H}-\mathrm{Au}$ bond to be more favorable than the adsorption to the Au-surface. This difficulty is illustrated in Fig. 3.4 where the fit's rms error is shown in relation to the H-Au binding energy (a) and the Au shear modulus (b): the fit with the lowest rms error is not necessarily the best one; it is necessary to pick a fit that reproduces the physical properties of the system satisfactorily while at the same time having a low rms error. 


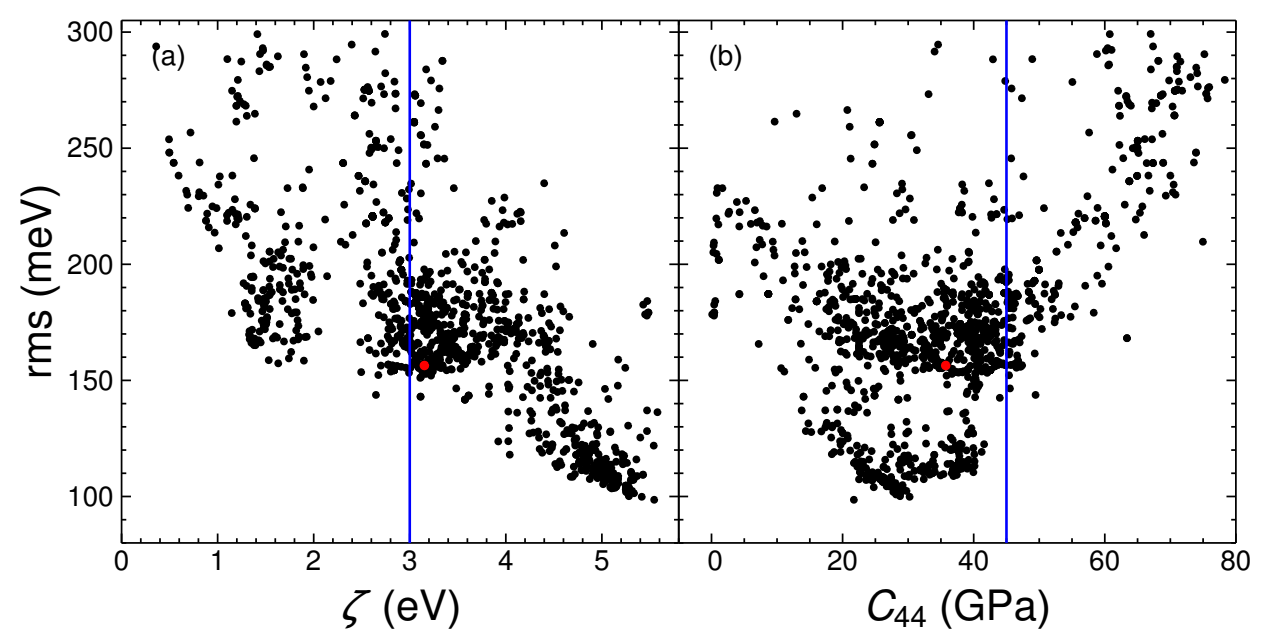

Figure 3.4.: rms error of the fit vs (a) H-Au bond energy $\zeta$ and (b) shear modulus for 1350 parametrizations (black points). The EMT-JAWK is indicated by a red point.

In Fig. 3.5 (a), the H-Au potential energy is shown as a function of the time during the doublebounce AIMD-trajectory (red circles), of which 200 points were used as input data for the fit, in comparison to the potential energy obtained with the EMT-JAWK (solid) for the same configurations. The panels (b)-(f) of Fig. 3.5 show the H-Au interaction energy as a function of time for configurations visited by eleven other AIMD trajectories (blue crosses) which were not used in the EMT fitting. For a testing set to judge the quality of the fit, I used the entire set of AIMD trajectories (that is, twelve AIMD trajectories not included into the fit and the one that was included in the fit. I did not excluded the AIMD trajectory used in the fit from the testing set to obtain a testing set that was the same for all fits, because for different fits, I used different AIMD trajectories as input). The corresponding potential energy obtained for the same configurations with EMT-JAWK are shown with solid lines. Panels (b)-(e) are results of singlebounce surface scattering trajectories, (f)-(j) of multibounce trajectories. For the trajectories in panels $(\mathrm{k})$ and $(\mathrm{l})$, the $\mathrm{H}$ atom penetrated below the surface and had not scattered back when the AIMD trajectories terminated after $120 \mathrm{fs}$ propagation time. The rms deviation to the testing set is $0.16 \mathrm{eV}$ and involves almost 9000 comparisons: a large volume of configuration space not sampled in the input data set of the fit is reproduced most satisfactorily.

To furthermore test how the fit compares to AIMD, I did electronically adiabatic MD simulations for the same incidence conditions as used by Kroes and coworkers [3, 4] for AIMD simulations of $\mathrm{H}$ scattering from $\mathrm{Au}(111)$ with an incidence energy of $E_{\text {inc }}=5.0 \mathrm{eV}$ and an

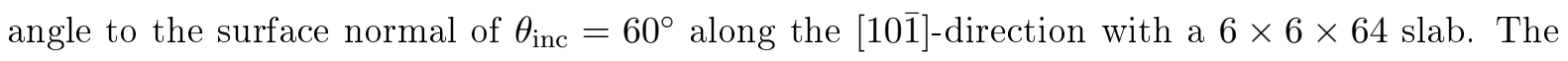
slab was thermalized to $120 \mathrm{~K}$ and the propagation time was $120 \mathrm{fs}$ with a time step of $0.1 \mathrm{fs}$. The comparison of the total energy loss distributions from both calculations in Fig. 3.6 shows that the EMT-MD calculations capture the features of the AIMD calculations, giving rise to a 


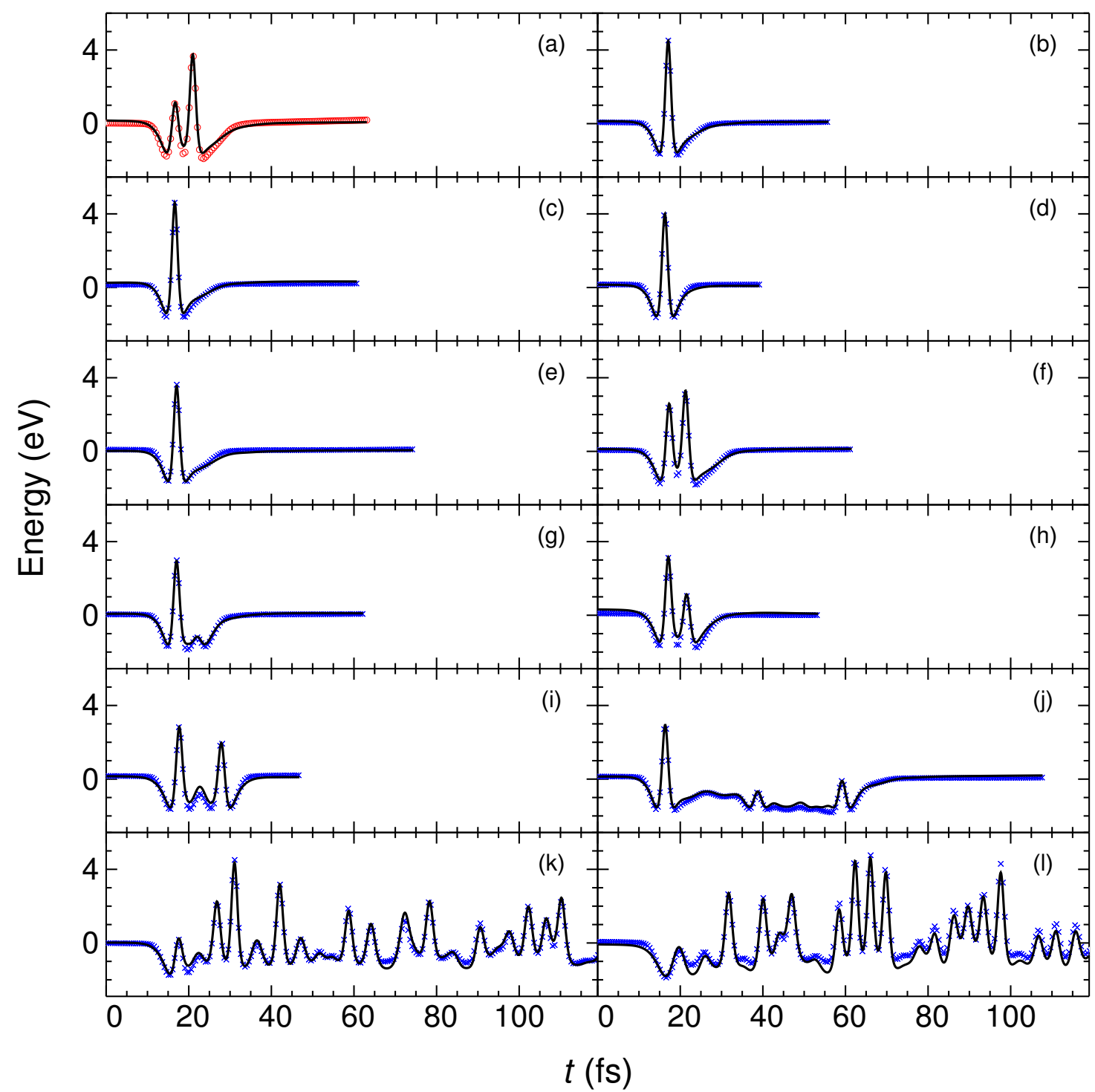

Figure 3.5.: Hydrogen-gold interaction energy profile along AIMD trajectories. Atom configurations and corresponding energy values from the AIMD trajectory ( $\circ$ ) shown in panel (a) were used as an input for the fitting procedure. The respective data from other AIMD trajectories $(\times)$ shown in panels (b)-(l) were used as a testing set to check the quality of the EMT fit (solid lines). The EMT-JAWK fitting function is capable of reproducing the the $a b i n i$ tio energy even for the configurations sampling the collision events which are qualitatively different from that in panel (a). With slight modifications reproduced from [22].

very similar peak structure at low energy losses and fall-off at higher energy losses. Scattering behavior can also be obtained. To calculate the probabilities, the number of those trajectories that undergo the process of interest are divided by the total number of trajectories $(N=900)$. Tab. 3.2 demonstrates that very good agreement is obtained for the $120 \mathrm{fs}$ simulations for all event probabilities (for details on classification of events, see Sec.2.8). What little difference 


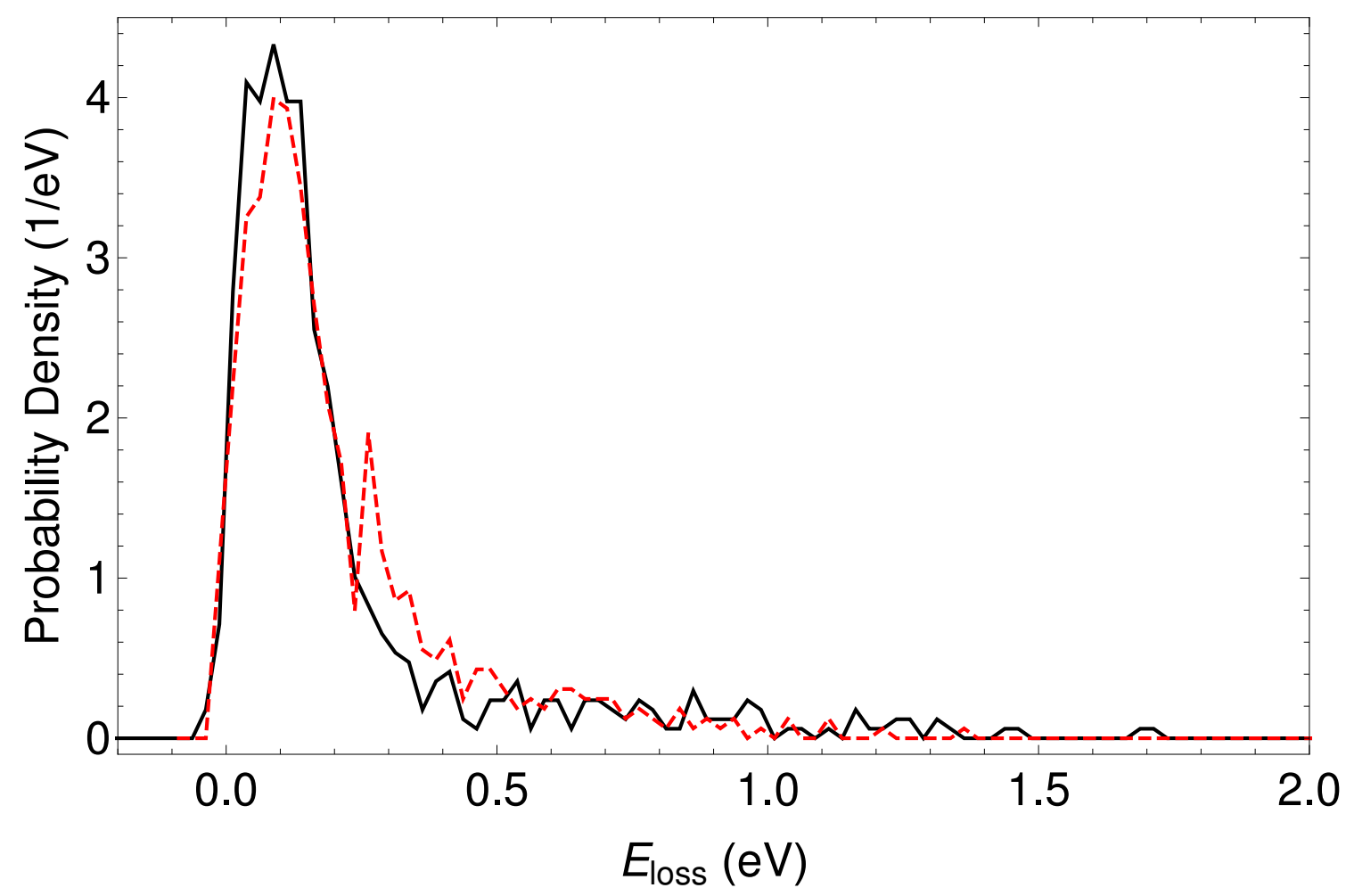

Figure 3.6.: Total energy loss distribution from adiabatic AIMD simulations [3, 4] (dashed line) and from adiabatic EMT-JAWK MD simulations (solid black line). Incidence conditions: $E_{\text {inc }}=5.0 \mathrm{eV}, \theta_{\text {inc }}=60^{\circ}$ along the $[10 \overline{1}]$-direction, 900 trajectories, $120 \mathrm{fs}$ propagation time. The Au slab was equilibrated to $120 \mathrm{~K}$. The binning interval was $\Delta E=25 \mathrm{meV}$.

there is can perhaps be attributed to the larger slab size $(6 \times 6 \times 64$ vs. $2 \times 2 \times 4)$ that is required for the EMT simulations compared to the AIMD simulations. Furthermore, for AIMD, simulations going far beyond a trajectory duration of $120 \mathrm{fs}$ or the calculation of a higher number of trajectories are, due to the large computational effort, almost unfeasible. Using EMT, the 900 trajectories presented here could be calculated in $\sim 150$ minutes on a single core. An extension of simulation time to 1 ps with EMT (Tab. 3.2) shows that the percentage of penetrating scattering trajectories is lower than the upper bound estimations of $35 \%$ and $42 \%$ for AIMD. Given the above comparison, it can be regarded as a good estimate of what AIMD might give for $1 \mathrm{ps}$ simulations, if the trajectories where the $\mathrm{H}$ atom passes through the slab are deemed to have no contribution to the total energy loss distribution.

From the analytic form of the EMT potential, the background electron density can be easily extracted and used to carry out nonadiabatic MD simulations on the level of LDFA [22]. The background electron density obtained at any position of the hydrogen atom is the electron density due to all surrounding $\mathrm{Au}$ atoms plus the disturbance caused by the presence of the $\mathrm{H}$ atom. In the DFT-calculations the electron density is not clearly separable, thus, the electron density used to be calculated as the electron density of the Au atoms without the $\mathrm{H}$ atom. In 
Table 3.2.: Outcomes (in \%) resulting from $\mathrm{H}$ atom collision with a $\mathrm{Au}(111)$ surface for adiabatic simulations for MD simulations with EMT-JAWK and AIMD [3, 4]. The incidence conditions are $E_{\text {inc }}=5.0 \mathrm{eV}, \theta_{\mathrm{inc}}=60^{\circ}$ along the $[10 \overline{1}]$ surface direction, with a relaxed surface structure, number of simulated trajectories: 900. The simulations were propagated for $120 \mathrm{fs}$.

\begin{tabular}{lllll}
\hline \hline & Scattering & with penetration & without penetration & transmission \\
\hline EMT & 74.9 & 18.2 & 56.7 & 6.89 \\
AIMD & $72.3 \pm 1.5$ & $14.0 \pm 1.2$ & $58.3 \pm 1.6$ & $7.3 \pm 0.9$ \\
EMT 1 ps & 87.8 & 26.1 & 61.8 & 10.6 \\
\hline \hline
\end{tabular}

simulations using LDFA, the electron density of EAM [30] and DFT [31] have both been used to obtain a friction coefficient.

The comparison between the $\mathrm{H}$ atom background electron density from EMT (black crosses) with the $a b$ initio electron density (red solid) in Fig. 3.7 shows that the background electron density from EMT compares well to the $a b$ initio density although being by a factor of $\approx 1.5$ higher inside the surface around the minima. In section 3.3.7 I explore the how electron density influences the scattering dynamics.

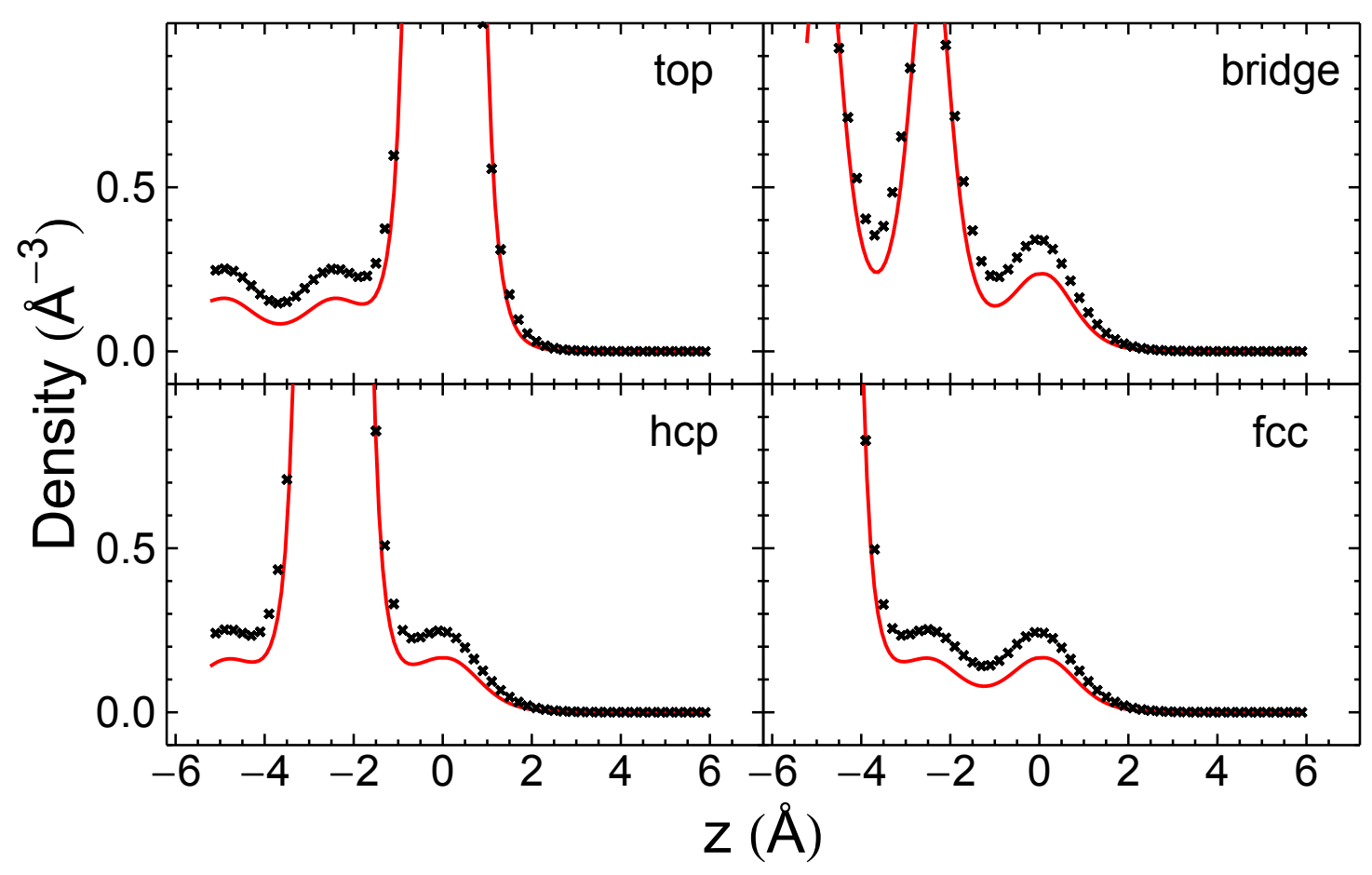

Figure 3.7.: The electron density as a function of the distance from the Au-surface extracted from the calculation with DFT (red solid) and the $\mathrm{H}$ atom background electron density from EMT-JAWK (black crosses) for a relaxed Au slab. The comparison is shown for the four symmetry sites. With slight modifications reproduced from [22]. 
Table 3.3.: Values of various physical properties for bulk Au calculated with the EMT-JAWK compared to the literature values. $\zeta$ is the energy of the $\mathrm{H}-\mathrm{Au}$ bond. Reproduced from $[22]$.

\begin{tabular}{llr}
\hline \hline & Fit & Lit. \\
\hline$a_{0}(\AA)$ & 4.20 & $4.08[177]$ \\
$C_{11}(\mathrm{GPa})$ & 195.8 & $201.6[125]$ \\
$C_{12}(\mathrm{GPa})$ & 160.1 & $169.7[125]$ \\
$C_{44}(\mathrm{GPa})$ & 35.94 & $45.4[125]$ \\
Bulk modulus $(\mathrm{GPa})$ & 172 & $173[234]$ \\
$\zeta(\mathrm{eV})$ & 3.2 & $3.0[229]$ \\
$T_{\text {stable }}(\mathrm{K})$ & 1000 & $1337[235]$ \\
\hline \hline
\end{tabular}

Tab. 3.3 shows that our EMT-JAWK reproduces physical properties of gold such as the shear modulus $\left(C_{44}\right)$, the other elastic constants $\left(C_{11}\right.$ and $\left.C_{12}\right)$ and the bulk modulus satisfactorily. A complete reproduction of the force constants is however not possible as the relation between $C_{11}$, $C_{12}$ and $C_{44}$ given by Eq. 2.51) is only true for isotropic crystals and not fulfilled by Au [124]. I obtained the temperature at which the slab becomes unstable by equilibrating the slab at successively higher temperatures in steps of $50 \mathrm{~K}$ for $20 \mathrm{ps}$ using a $6 \times 6 \times 6$ cell, and took the highest temperature at which the slab did not disintegrate as $T_{\text {stable }}=1000 \mathrm{~K}$. This temperature might, on first glance, appear much too low, but experiment shows that the topmost layers of the surface disorder at $\approx 1070 \mathrm{~K}$, leaving no clear surface structure at higher temperatures [183]. The EMT-JAWK reproduces the hydrogen bond energy $\zeta$ within $0.2 \mathrm{eV}$ and the deviation of the lattice constant results from taking the lattice constant from the DFT input data [21] for the self-consistency of the fitting.

\subsubsection{Comparison to earlier EMT parametrizations}

Strömquist et al. [23] and Jacobsen et al. [2] published previously the EMT parametrization for $\mathrm{Au}$ and $\mathrm{H}$ (see Tab. 3.1, EMT-JS). Jacobsen et al. obtained the parameters for Au by relying on experimentally measured properties of Au such as bulk modulus, elastic moduli and lattice constant [2. As mentioned in section 3.2, the lattice constant for Au derived with DFT-GGA functionals does not agree with the experimentally observed lattice constant of $\mathrm{Au}$; for the following comparison I therefore modified Jacobsen's value of $s_{0, A u}$ from $1.588 \AA$ to $1.642 \AA$ to achieve an agreement with the lattice constant from the $a b$ initio calculations. This modified Jacobsen-Strömquist parameterization (EMT-mJS) does not reproduce the 3D-grid having an rms error $1130 \mathrm{meV}$ to it, and an rms error of $939 \mathrm{meV}$ to the points taken from the AIMD calculations. In Fig. 3.8 a comparison between the EMT-JAWK (black solid) and EMT-mJS (purple solid) to the $a b$ initio input data set for four symmetry sites is shown. It shows that 


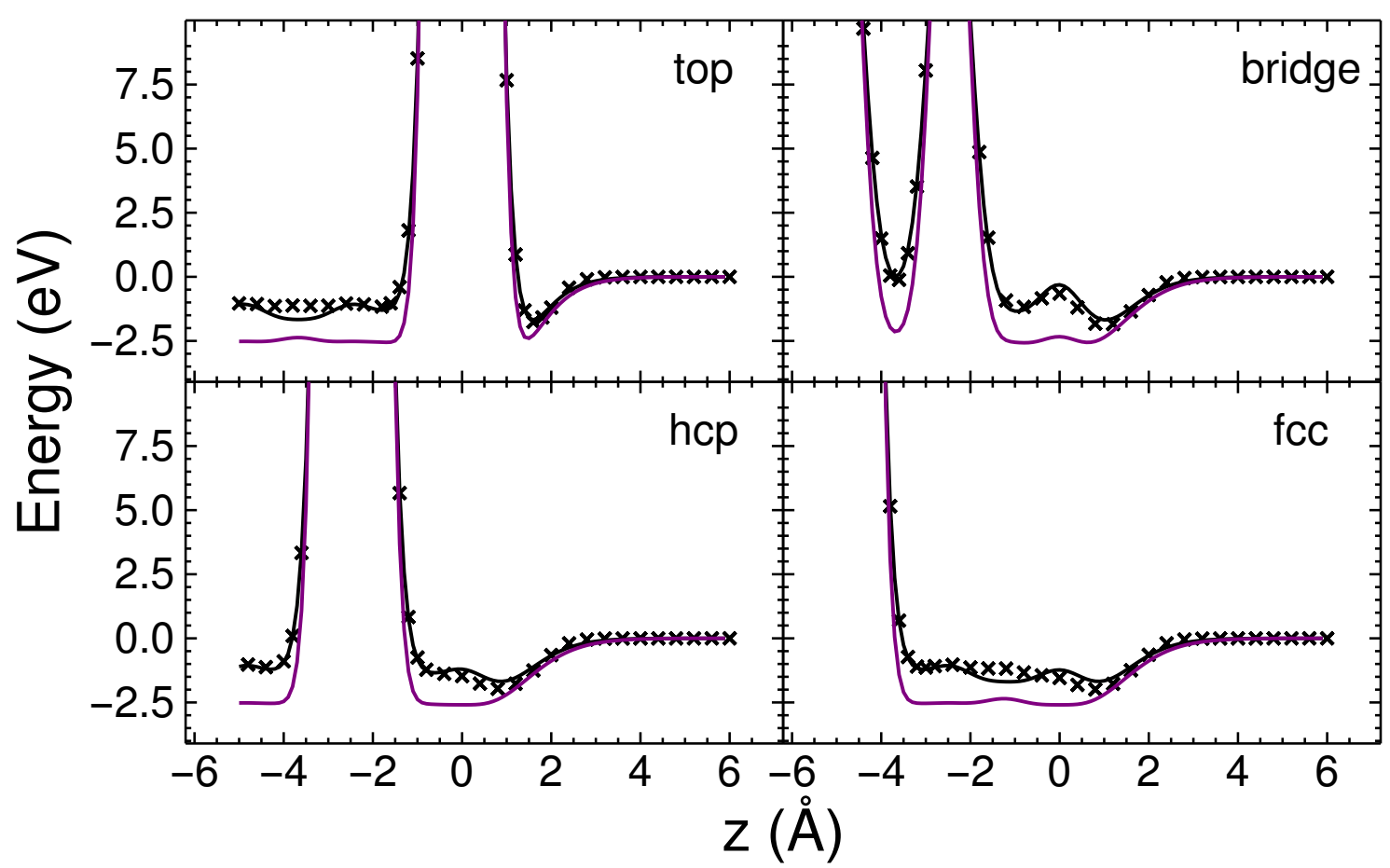

Figure 3.8.: Dependence of the $\mathrm{H}$-Au interaction energy on $z$-distance of the $\mathrm{H}$ atom from the surface for four symmetry sites (top, bridge, hcp hollow and $f c c$ hollow). The results of ab initio GGA-SRP48 calculations (black cross) are compared to the EMT-JAWK (black solid line) and the EMT-mJS given by the parametrization of Stroemquist [23] and Jacobsen [2] with a modified $s_{0, A u}$ in agreement with the lattice constant of the $a b$ initio calculations (purple solid line).

the EMT-mJS parametrization is significantly far off from the $a b$ initio values. The minima are deeper and broader than the adsorption minima of EMT-JAWK, but the background electron density obtained with EMT-mJS is very similar to the background electron density corresponding to EMT-JAWK (see Fig.3.9). To test if the EMT-mJS does indeed lead to much different dynamic behavior than the EMT-JAWK, I did molecular dynamics simulations both with and without the inclusion of electron hole pair excitation. The incidence conditions for all simulations were: $E_{\text {inc }}=3.33 \mathrm{eV}, \theta_{\text {inc }}=45^{\circ}$ along the $[10 \overline{1}]$ surface direction, $T=300 \mathrm{~K}$ with a $6 \times 6 \times 6$ slab and $10^{5}$ trajectories. As can be seen from Tab. 3.4, the scattering behavior resulting from both fits differs considerably; in accordance with the deep ad- and absorption minima for the EMT-mJS, the scattering probability for both adiabatic and nonadiabatic MD-simulations is much lower than for EMT-JAWK as is the adsorption probability. Transmission through the surface is more likely with the EMT-mJS than with EMT-JAWK and the absorption into the surface is much higher than for EMT-JAWK if energy loss to electron hole pairs is included into the MD-simulations. In total, the dynamical behavior of the EMT-mJS is much different from that of EMT-JAWK, as is further emphasized by comparison of the total energy loss distribution 


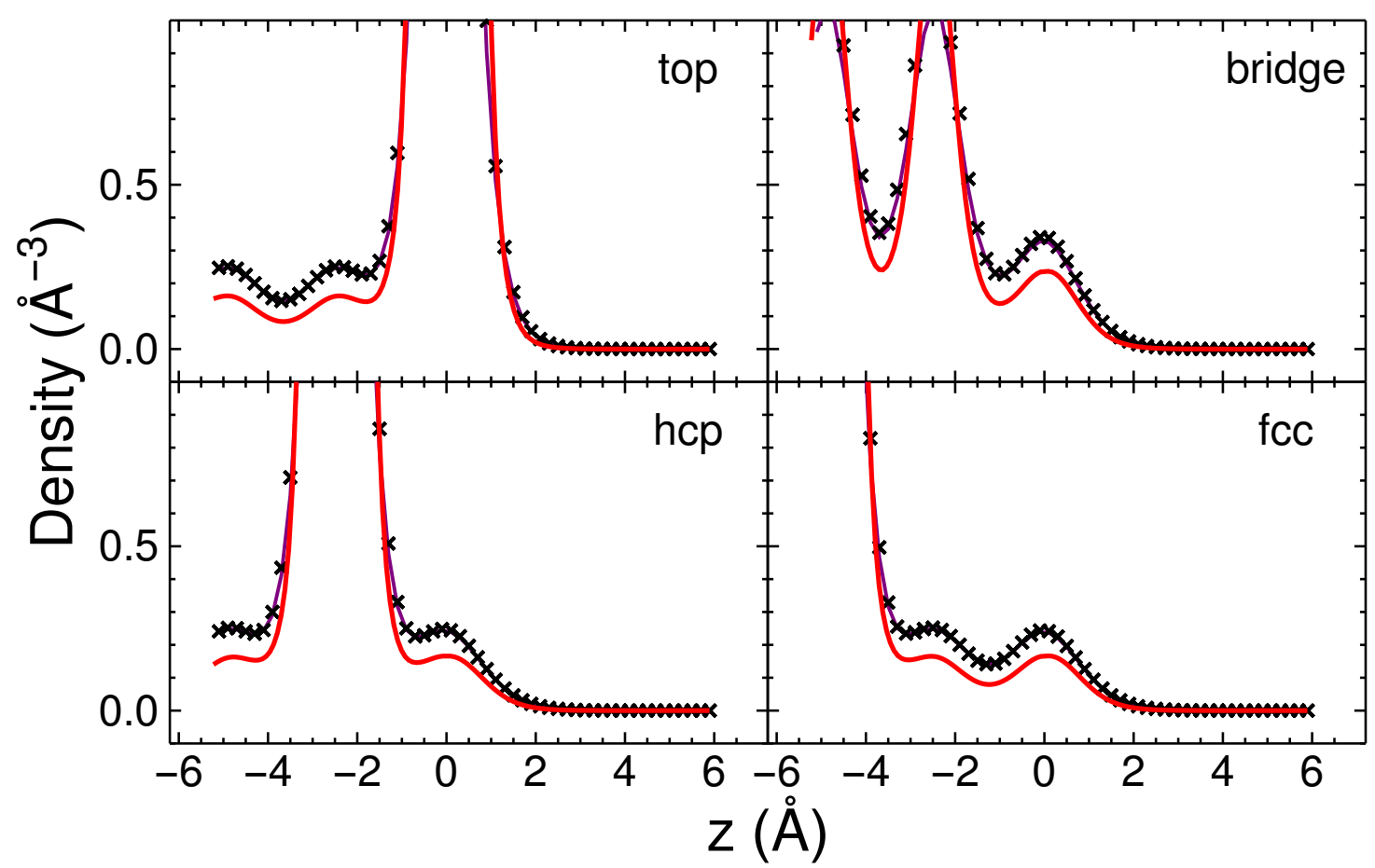

Figure 3.9.: Electron density extracted from DFT calculation with a relaxed Au slab (red solid) and the $\mathrm{H}$ atom background electron density from EMT-JAWK (black crosses) and from EMTmJS (purple solid). The comparison is shown as a function of the $\mathrm{H}$ atom $z$ coordinate for four symmetry sites.

Table 3.4.: Various outcomes (in \%) resulting from $\mathrm{H}$ atom collision with a $\mathrm{Au}(111)$ surface for nonadiabatic and adiabatic (values in parenthesis) MD-simulations using EMT-JAWK and EMTmJS. The incidence conditions are $E_{\text {inc }}=3.33 \mathrm{eV}, \theta_{\text {inc }}=45^{\circ}$ along the $[10 \overline{1}]$ surface direction, $T=300 \mathrm{~K}$ and a $6 \times 6 \times 6$ slab $\left(10^{5}\right.$ trajectories $)$.

\begin{tabular}{lllll}
\hline \hline & Scattering & $\begin{array}{l}\text { Surface } \\
\text { adsorption }\end{array}$ & $\begin{array}{l}\text { Subsurface } \\
\text { absorption }\end{array}$ & Transmission \\
\hline EMT-JAWK & $55(82)$ & $23(4)$ & $22(6)$ & $0(8)$ \\
\hline EMT-mJS & $32(77)$ & $8(0)$ & $58(2)$ & $2(21)$ \\
\hline \hline
\end{tabular}

for both PESs when accounting for electron hole pair excitation (Fig. 3.10): the total energy loss distribution resulting from the EMT-mJS (purple) shows a much more pronounced first peak and a very differently shaped tail than the total ELD of EMT-JAWK, although the position of the energy loss peak is not much affected by the choice of the parametrization. This can be attributed to the fact that the energy loss to electron hole pairs prevails over the adiabatic one, since the energy loss to ehp is governed by the background electron density, the lack of difference between EMT-JAWK and EMT-mJS in the position of the first peak is comprehensible. 


\subsubsection{Comparison of different fits}

As already mentioned in section 3.2, obtaining a parametrization that has both a low rms error value and fits the constraints is far from trivial. To obtain parametrizations that fit the constraints better than the EMT-JAWK presented here, I tried several strategies. I tested how the fit depended on the input data set belonging to the 3D-grid; first I checked if the number of symmetry sites included redundant sites which did not appear to be the case as the fit does not improve with exclusion of the symmetry sites but tends to worsen. Second, I tested how the fit depended on excluding the minima in potential energy from the 3D-grid input data set. I did this because I noticed during previous fits that the better the minima are represented, the lower is the shear modulus and the higher is the $\mathrm{H}-\mathrm{Au}$ bond energy. Indeed, the more points with a low potential energy were excluded, the better the representation of physical properties of the $\mathrm{Au}$ became. Surprisingly, the rms error to all AIMD trajectories did not worsen but remained around $160 \mathrm{meV}$. However, if only the potential energy walls around the atom cores were fitted, the shear modulus became too high and the H-Au bond too weak. Increasing the energy cut-off to include higher values in potential energy up to $25 \mathrm{eV}$ compared to $20 \mathrm{eV}$ for the fit resulting in the EMT-JAWK parametrization also improved the representation of the physical properties

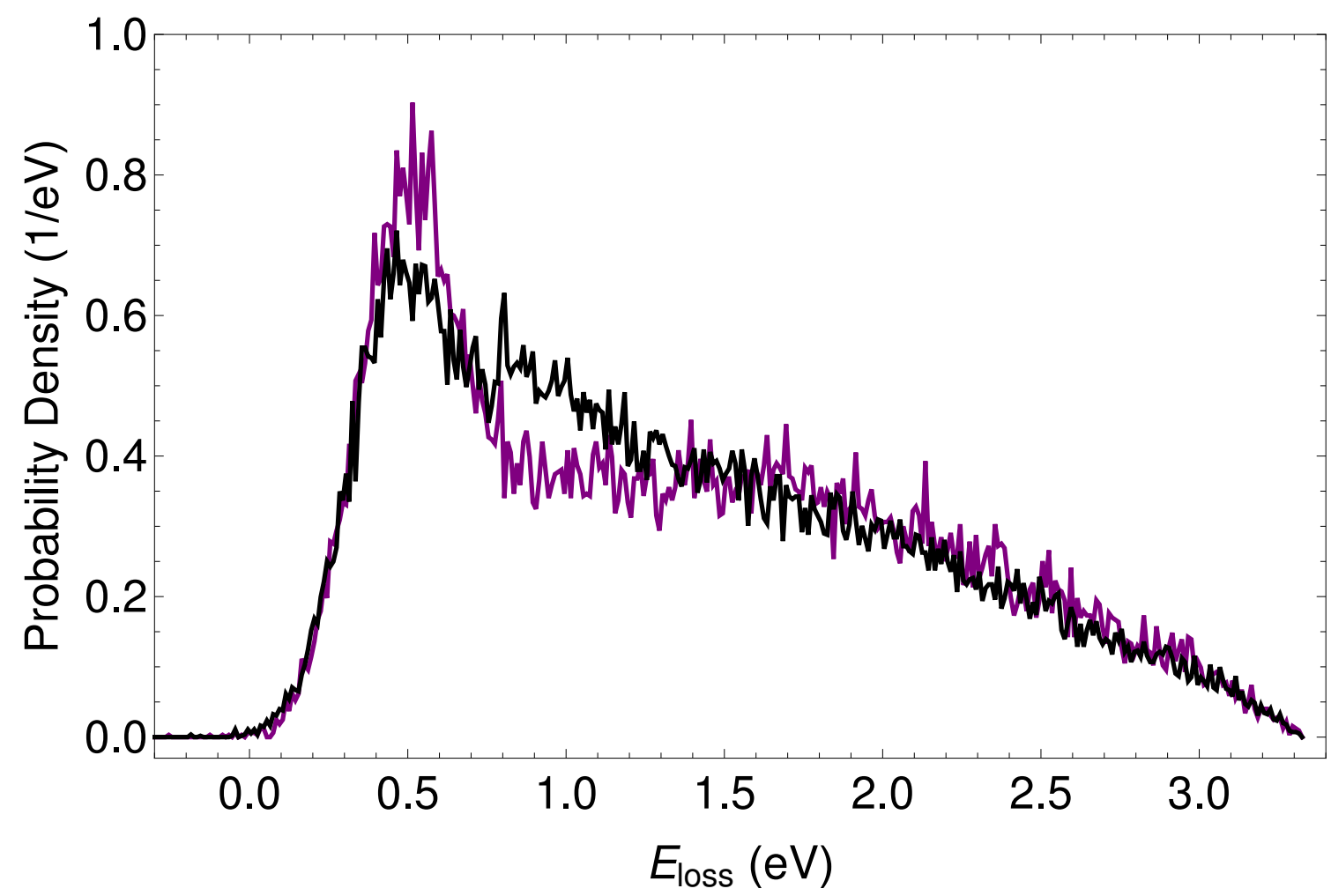

Figure 3.10.: Total energy loss distribution of nonadiabatic MD-simulations using EMT-JAWK (black) and EMT-mJS (purple). 


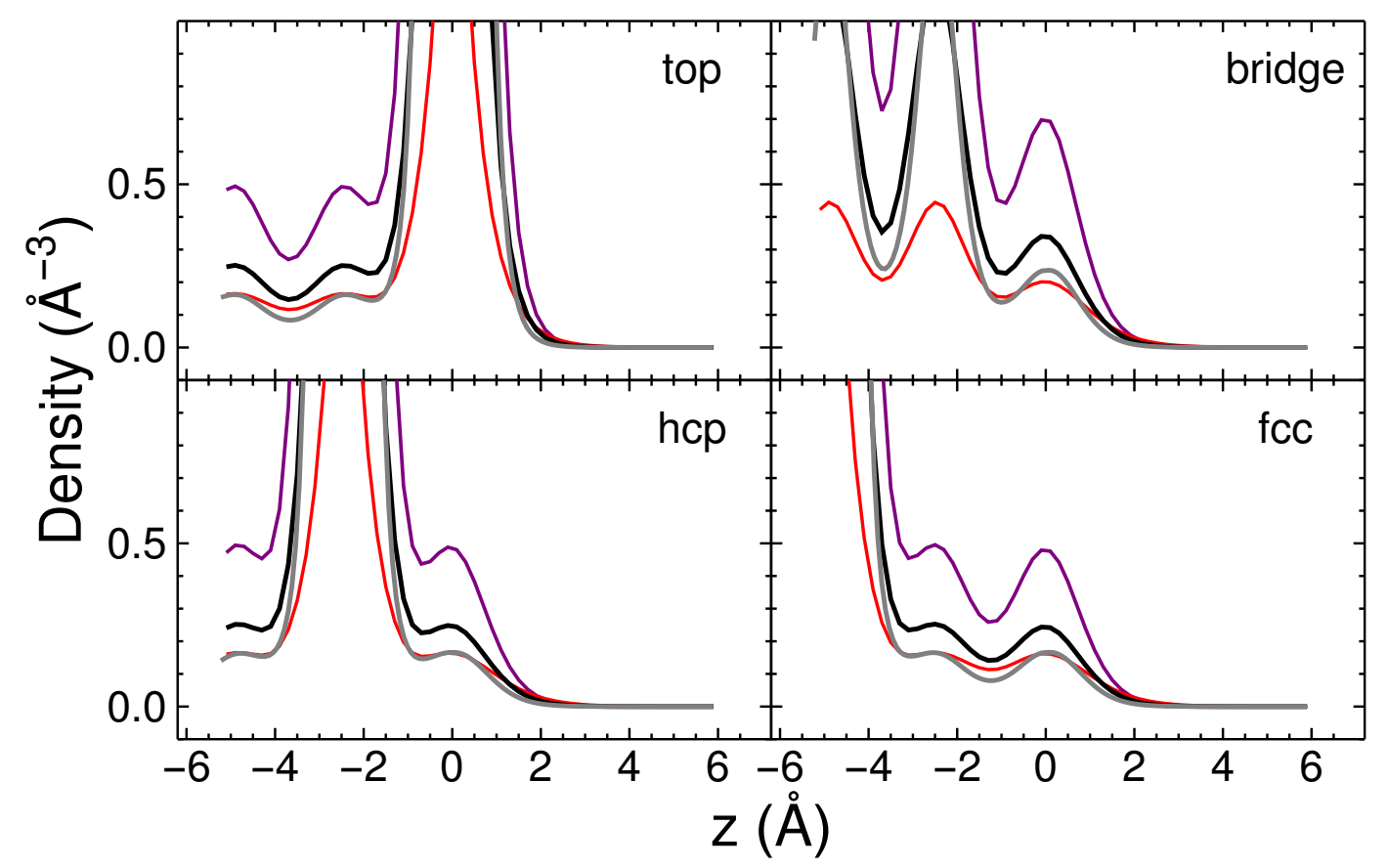

Figure 3.11.: Background electron density for four high symmetry sites as a function of the $\mathrm{H}$ atom distance to the Au surface $z$ for the fits 894 (purple), 921 (red) and EMT-JAWK (thick, black), and the electron density obtained from DFT (thick, grey).

of $\mathrm{Au}$ and the $\mathrm{H}-\mathrm{Au}$ bond energy, most likely by virtue of reducing the weight on the points representing the structure of the minima.

There are two further constraints which I would suggest for future fitting processes. First, Jacobsen et al. [2] write in their description of the EMT that only $\delta_{\text {metal }} / \eta_{\text {metal }} \ll 1$ is the acceptable range for $\delta_{\text {metal }}$. Furthermore, the background electron density that results from the fit should be monitored to avoid too high or too low values. While the first case is most likely caused by a too low $s_{0, \mathrm{H}}$, I was not able to establish a straightforward relation between the parameters that could explain very large background electron densities, since the relation of the parameters that give rise to the background electron density is rather complex (see Eq. 2.30). Although the electron density that can be obtained with DFT-GGA functionals differs from the background electron density obtained from EMT by not including the feedback caused by the presence of the $\mathrm{H}$ atom, it still can be used as an indication of whether the background electron density obtained from a fit is reasonable: if the background electron density is (i) much higher in the space between the Au atoms or (ii) much lower around the atom cores than the electron density from DFT calculations, the fit should be discarded. As shown in the two examples presented below, case (i) leads to almost complete sticking to the surface. Case (ii) leads to unphysical behavior: the $\mathrm{H}$ atom may not see an increase in electron density upon approaching the $\mathrm{Au}$ atoms. 
Table 3.5.: Values of various physical properties for bulk Au calculated with the different fits compared to the literature values. $\zeta$ is the energy of the $\mathrm{H}-\mathrm{Au}$ bond.

\begin{tabular}{lllllll}
\hline \hline & Fit 894 & Fit 921 & Fit 1138 & Fit 1226 & Fit 1288 & Lit. \\
\hline rms error to & & & & & & \\
AIMD $(\mathrm{meV})$ & 170 & 160 & 150 & 160 & 160 & \\
$C_{11}(\mathrm{GPa})$ & 205.0 & 198.8 & 200.0 & 201.8 & 202.4 & $201.6[125]$ \\
$C_{12}(\mathrm{GPa})$ & 162.0 & 158.6 & 158.0 & 157.1 & 156.8 & $169.7[125]$ \\
$C_{44}(\mathrm{GPa})$ & 43.05 & 40.21 & 42.1 & 44.75 & 45.6 & $45.4[125]$ \\
Bulk modulus $(\mathrm{GPa})$ & 172 & 172 & 172 & 172 & 172 & $173[234]$ \\
$\zeta(\mathrm{eV})$ & 2.8 & 2.9 & 3.0 & 3.1 & 3.1 & $3.0[229]$ \\
$T_{\text {stable }}(\mathrm{K})$ & 1250 & 1150 & 1100 & 1150 & 1050 & $1337[235]$ \\
\hline \hline
\end{tabular}

While trying to optimize the fitting procedure, I obtained a large number of fits of which several fulfil the physical constraints I have chosen for EMT-JAWK. Here, I give a comparison between five different fits that all have an rms error below $185 \mathrm{meV}$ and otherwise fulfil the physical constraints described in the previous section. I identify the parametrizations by their fit number and chose fits 894 and 921 (see Tab. 3.5) to point out that for both of them the physical constraints appear to be met, but the background electron density takes too large or too low values (see Fig. 3.11, purple and red, respectively); for both fit 894 and fit $921 \delta_{\text {metal }} / \eta_{\text {metal }}>1$, too.

I obtained several other EMT parametrizations (Tab 3.5, fits 1138, 1226 and 1288) displaying low rms errors, good agreement with the elastic moduli and $\zeta$ (see Tab. 3.5). I consider all three of them as examples for potential candidates for a PES that reproduce the bulk properties of $\mathrm{Au}$ better than EMT-JAWK and could be used if the EMT-JAWK should turn out unsatisfactory in future. The difference between the last three fits is in their respective input data sets: for fit 1138, I used a single bounce trajectory as input data set from the AIMD trajectories. For fit 1226, I allowed the inclusion of all points in the input data set that had a potential energy below $25 \mathrm{eV}$ instead of $20 \mathrm{eV}$ as a maximal energy cut-off, and for fit $1288 \mathrm{I}$ excluded the 240 points with the lowest potential energy values of the $3 \mathrm{D}$-grid input data. The values of the parameters to the fits are listed in Tab. 3.6 .

To test how the different parametrizations influence the $\mathrm{H}$ atom scattering I performed electronically adiabatic and nonadiabatic molecular dynamics simulations. The incidence conditions for all simulations were: $E_{\text {inc }}=3.33 \mathrm{eV}, \theta_{\text {inc }}=45^{\circ}$ along the $[10 \overline{1}]$ surface direction, $T=300 \mathrm{~K}$ and $6 \times 6 \times 6$ slab $\left(10^{5}\right.$ trajectories). Tab. 3.7 shows a comparison of the various outcomes of the simulations for the different fits. For the electronically adiabatic case (given in parenthesis), all fits display much the same results. For the nonadiabatic case, the differences between EMT- 
JAWK, fit 1138, 1226 and 1288 are almost negligible, whereas for fit 894, all $\mathrm{H}$ atoms stick to the surface and fit 921 brings many more $\mathrm{H}$ atoms to adsorb to the surface than to subsurface regions.

A comparison of the total energy loss distributions shows great similarity for the electronically adiabatic case (Fig. 3.12(a)). In the electronically nonadiabatic case, fit 894 does not appear as, according to Tab. 3.7, no $\mathrm{H}$ atoms scatter if interaction with electron hole pairs is included. The

Table 3.6.: Fit Parameters defining H-Au EMT PESs.

\begin{tabular}{llllllll}
\hline \hline & $\eta_{2}\left(\AA^{-1}\right)$ & $n_{0}\left(\AA^{-3}\right)$ & $\varepsilon_{0}(\mathrm{eV})$ & $\lambda\left(\AA^{-1}\right)$ & $V_{0}(\mathrm{eV})$ & $\kappa\left(\AA^{-1}\right)$ & $s_{0}(\AA)$ \\
\hline Fit 894 & & & & & & \\
\hline $\mathrm{Au}$ & 3.485 & 0.064 & $-3.8[2]$ & 4.233 & 0.378 & 2.7531 & 1.642 \\
$\mathrm{H}$ & 5.404 & 0.408 & $-2.371[23]$ & 8.036 & 0.244 & 9.752 & 0.674 \\
\hline Fit 921 & & & & & & \\
\hline $\mathrm{Au}$ & 3.308 & 0.061 & $-3.8[2]$ & 4.182 & 0.391 & 2.629 & 1.642 \\
$\mathrm{H}$ & 1.235 & 0.184 & $-2.371[23]$ & 1.741 & 0.335 & 3.287 & 0.480 \\
\hline Fit 1138 & & & & & & \\
$\mathrm{Au}$ & 3.200 & 0.047 & $-3.8[2]$ & 4.182 & 0.460 & 3.630 & 1.642 \\
$\mathrm{H}$ & 4.320 & 0.181 & $-2.371[23]$ & 7.133 & 0.229 & 7.588 & $0.680[23]$ \\
\hline Fit 1226 & & & & & & \\
$\mathrm{Au}$ & 3.096 & 0.051 & $-3.8[2]$ & 4.182 & 1.114 & 4.898 & 1.642 \\
$\mathrm{H}$ & 4.692 & 0.167 & $-2.371[23]$ & 7.855 & 0.359 & 8.322 & $0.680[23]$ \\
\hline Fit 1288 & & & & & & \\
$\mathrm{Au}$ & 3.101 & 0.051 & $-3.8[2]$ & 4.182 & 1.193 & 4.948 & 1.642 \\
$\mathrm{H}$ & 4.787 & 0.167 & $-2.371[23]$ & 7.940 & 0.364 & 8.496 & $0.680[23]$ \\
\hline \hline
\end{tabular}
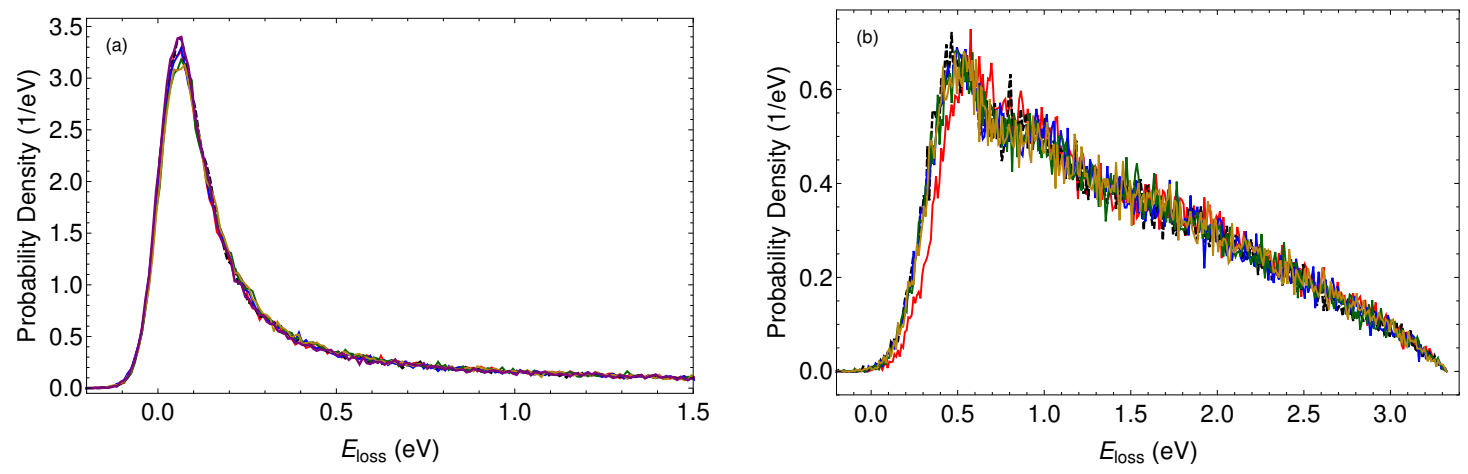

Figure 3.12.: Total energy loss distribution for (a) adiabatic and (b) nonadiabatic scattering for the fits 894 (purple), 921 (red), 1138 (blue), 1226 (dark green) and 1288 (dark yellow) and EMT-JAWK (black, dashed). 
Table 3.7.: Various outcomes (in \%) resulting from $\mathrm{H}$ atom collision with a $\mathrm{Au}(111)$ surface for nonadiabatic and adiabatic (in parenthesis) simulations using various fits. The incidence conditions are $E_{\text {inc }}=3.33 \mathrm{eV}, \theta_{\text {inc }}=45^{\circ}$ along the $[10 \overline{1}]$ surface direction, $T=300 \mathrm{~K}$, a $6 \times 6 \times 6$ slab and number of trajectories $10^{5}$.

\begin{tabular}{|c|c|c|c|c|}
\hline conditions & Scattering & $\begin{array}{l}\text { Surface } \\
\text { adsorption }\end{array}$ & $\begin{array}{l}\text { Subsurface } \\
\text { absorption }\end{array}$ & Transmission \\
\hline EMT-JAWK & $55(82)$ & $23(4)$ & $22(6)$ & $0(8)$ \\
\hline Fit 894 & $0(82)$ & $93(4)$ & $6(6)$ & $0(9)$ \\
\hline Fit 921 & $57(82)$ & $32(6)$ & $10(4)$ & $1(7)$ \\
\hline Fit 1138 & $54(81)$ & $23(4)$ & $23(7)$ & $0(8)$ \\
\hline Fit 1226 & $52(80)$ & $22(4)$ & $25(7)$ & $0(8)$ \\
\hline Fit 1288 & $52(80)$ & $22(4)$ & $26(8)$ & $1(8)$ \\
\hline
\end{tabular}

reason for this behavior becomes evident from Fig. 3.11; the background electron density for fit 894 (purple) is so high that the friction coefficient in Eq. 2.53 becomes so large that the $\mathrm{H}$ atoms lose much of their initial energy and stick to the surface.

The total energy loss distribution in case of fit 921 (Fig 3.12 (b), red) is shifted to higher energy values compared to the other fits. In view of the much lower background electron density (Fig. 3.11, red), this is surprising, but it might be caused by different scattering due to the $\mathrm{H}$ atoms being able to come closer to the Au atoms which is made possible by the less steep rise in electron density close to the Au atoms. However, as the repulsive interaction with the atomic cores should be caused by electron-electron repulsion between the electron of the $\mathrm{H}$ atom and those of the $\mathrm{Au}$ atom, the lack of increasing electron density upon getting closer to an $\mathrm{Au}$ atom is clearly wrong. This failing can most likely be attributed to the too low value of the

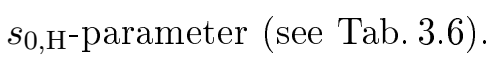

For the fits 1138, 1226, 1288 and the one resulting in EMT-JAWK the total energy loss distributions for both the adiabatic (Fig. 3.12(a)) and nonadiabatic (Fig. 3.12(b)) case agree well, as do their respective electron densities (Fig 3.13). The scattering probabilities show only very small differences. The percentage of single bounces in all scattering trajectories is also very similar, lying between $25-26 \%$, as is that of the double-bounces $(34-36 \%)$ and multibounces $(39-43 \%)$. The percentage of penetrating trajectories varies between $20-24 \%$.

A comparison of the differential energy loss distributions for all four fits in specular scattering angle (see Fig. 3.14 shows that all fits give rise to the same DELD. It appears therefore that the translational energy loss for the adiabatic and nonadiabatic case as well as the sticking behaviour is not much influenced by the choice of the parameter set if all constraints are met (including the additional ones mentioned in this section). 


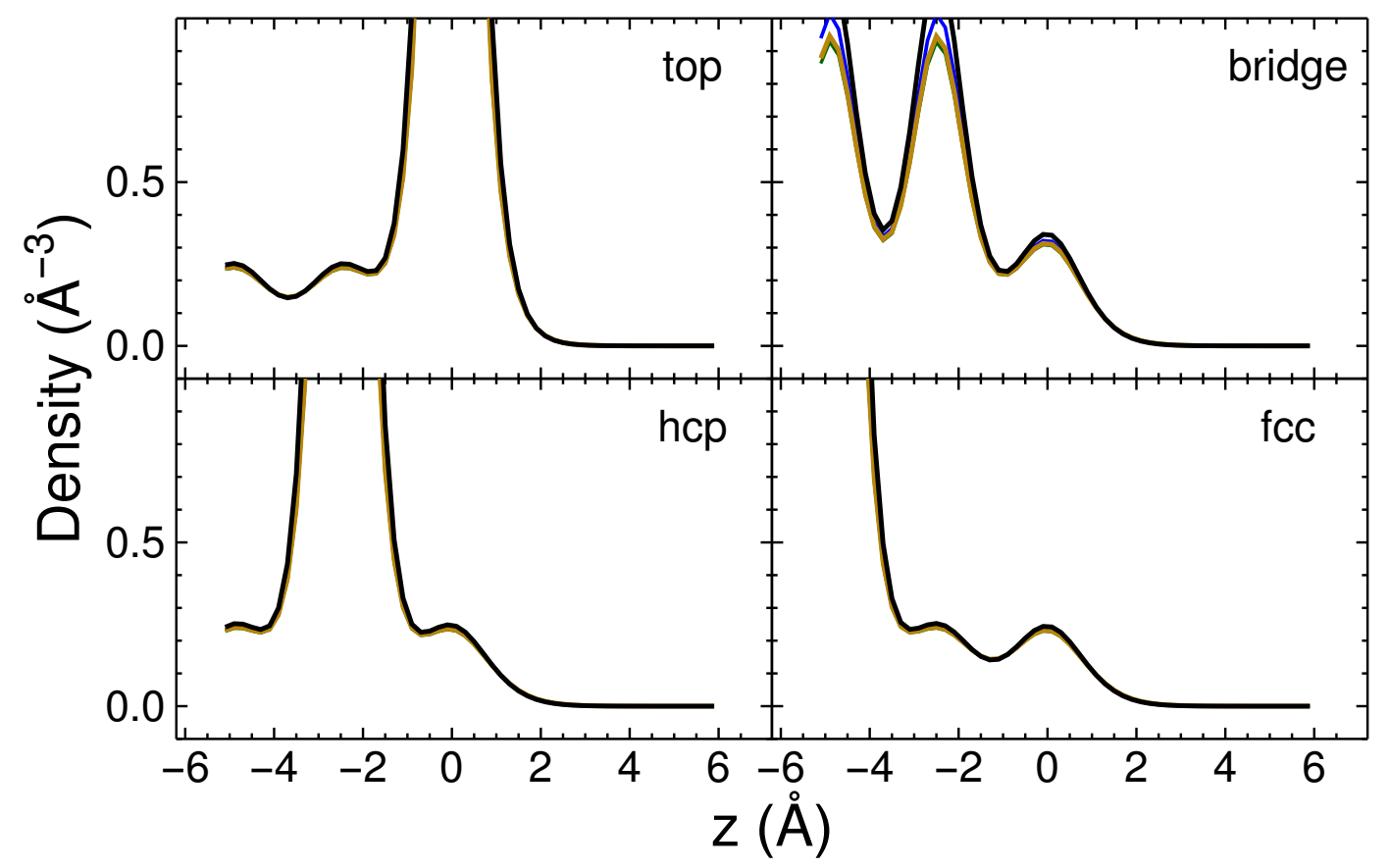

Figure 3.13.: Background electron densities for four high symmetry sites as a function of the $H$ atom distance to the Au-surface for the fits 1138 (blue), 1226 (dark green), 1288 (dark yellow) and fit 1 (black, thick).

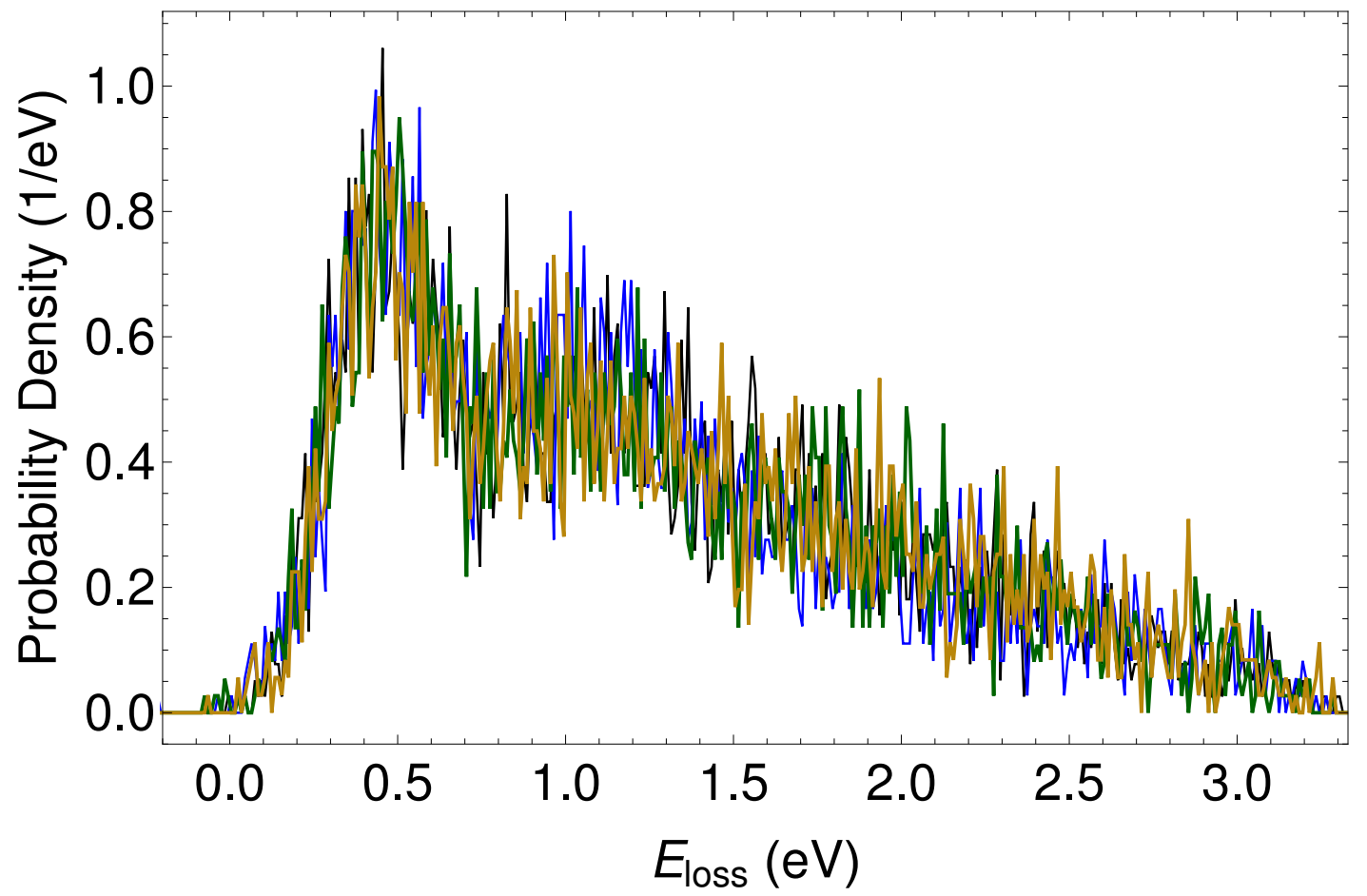

Figure 3.14.: Differential energy loss distribution for fits EMT-JAWK (black), 1138 (blue), 1126 (dark green) and 1188 (dark yellow) for specular scattering extracted from $10^{6}$ simulated trajectories. 


\subsection{Simulation of $\mathrm{H}$ scattering from $\mathrm{Au}(111)$ with Various Conditions}

One of the purposes of performing MD simulations of $\mathrm{H}$ scattering from $\mathrm{Au}(111)$ is to identify scattering conditions that are particularly remarkable in their scattering mechanism. These scattering conditions can then be investigated in the experiment and the results from the experiment compared to the results from the MD simulations and explained by them. I use MD simulations to predict scattering conditions that are particularly interesting to investigate experimentally.

I have sampled the behavior of scattering of $\mathrm{H}$ from $\mathrm{Au}(111)$ in the temperature range from 0 to $700 \mathrm{~K}$, under $\theta_{\text {inc }}=0^{\circ}$ to $60^{\circ}$ along the $[10 \overline{1}]$ and $[11 \overline{2}]$ surface directions, with a reconstructed and relaxed surface and a surface with an ad-atoms and six or four layers for the adiabatic and nonadiabatic case.

To compare different conditions during molecular dynamics simulations to one another, I have chosen the MD-simulation of $E_{\text {inc }}=3.33 \mathrm{eV}, \theta_{\text {inc }}=45^{\circ}$ along the [101] surface direction at $300 \mathrm{~K}$ with a relaxed surface structure and $6 \times 6 \times 6$ cell and $10^{6}$ trajectories as reference incidence conditions. By this I mean that when I studied the behavior with e.g. varying temperature, I kept all the other conditions as described in the previous sentence. The simulation time has been $1 \mathrm{ps}$, enough time to decide the outcome of a trajectory, and time step was $0.1 \mathrm{fs}$.

\subsubsection{Detailed analysis of scattering outcomes}

All simulations in this section refer to the following incidence conditions: $E_{\text {inc }}=3.33 \mathrm{eV}, \theta_{\text {inc }}=$ $45^{\circ}$ along the $[10 \overline{1}]$ surface direction, $T=300 \mathrm{~K}$ with a relaxed surface and $6 \times 6 \times 6$ slab, number of simulated trajectories: $10^{6}$ for a simulation time of 1 ps. Tab. 3.8 shows the probabilities for $\mathrm{H}$ scattering from $\mathrm{Au}(111)$. The electronically adiabatic results show high scattering and low absorption probability: the probability for adsorption to the surface and absorption into the slab is only slightly higher than the probability for transmission through the 6-layered slab. In contrast, if electron hole pair effects are included, the probability of absorption is enhanced by a factor of 3.5, and that to the surface almost by a factor of six compared to the electronically adiabatic case; transmission through the slab is also much less likely. In short, the inclusion of

Table 3.8.: Various outcomes (in \%) resulting from $\mathrm{H}$ atom collision with a $\mathrm{Au}(111)$ surface for nonadiabatic and adiabatic simulations.

\begin{tabular}{lllll}
\hline \hline & & Surface & Subsurface & \\
Conditions & Scattering & Adsorption & Absorption & Transmission \\
\hline nonadiabatic & 55 & 23 & 21 & 1 \\
adiabatic & 82 & 4 & 6 & 8 \\
\hline \hline
\end{tabular}


Table 3.9.: Pathways (in \%) of sticking trajectories for nonadiabatic and adiabatic (in parenthesis) simulations resolved according to deepest penetration and final position of $\mathrm{H}$ atom. Roman numerals denote the sub-surface layer.

\begin{tabular}{|c|c|c|c|c|c|c|c|c|}
\hline \multicolumn{9}{|c|}{ Deepest penetration } \\
\hline final & surf & I & II & III & IV & $\mathrm{V}$ & $>\mathrm{V}$ & Total \\
\hline surf & $10(0)$ & $29(1)$ & $10(6)$ & $1(8)$ & $0(3)$ & $0(2)$ & $0(0)$ & $51(20)$ \\
\hline $\mathrm{I}$ & & $6(0)$ & $5(2)$ & $1(3)$ & $0(2)$ & $0(1)$ & $0(0)$ & $12(8)$ \\
\hline II & & & $14(2)$ & $8(8)$ & $1(4)$ & $0(2)$ & $0(0)$ & $23(16)$ \\
\hline III & & & & $8(5)$ & $1(2)$ & $0(1)$ & $0(0)$ & $9(8)$ \\
\hline IV & & & & & $3(1)$ & $0(0)$ & $0(0)$ & $3(1)$ \\
\hline $\mathrm{V}$ & & & & & & $1(0)$ & $0(0)$ & $1(0)$ \\
\hline$>\mathrm{V}$ & & & & & & & $1(45)$ & $1(45)$ \\
\hline
\end{tabular}

electron hole pair excitation causes many more $\mathrm{H}$ atoms to stick to the Au-surface.

When considering the dynamics of how the sticking $\mathrm{H}$ atoms end up after $1 \mathrm{ps}$ in their respective position, the difference between electronically adiabatic and nonadiabtic simulations become even more evident (see Tab. 3.9 ): in the adiabatic case, it is most favorable to pass deep into or through the six-layered slab; adsorption only occurs for $20 \%$ of all non-scattered atoms at the end of the simulation time and of these, most penetrated the slab until the third sublayer before they resurfaced. In contrast, for the nonadiabatic simulations, it is most likely for a $\mathrm{H}$ atom to end up at the surface. Atoms that populate the surface are most likely to do so by subsurfacing to the first sublayer and then resurfacing again; however, $1 / 5^{\text {th }}$ of the atoms that end up populating the surface lost all their energy upon collision with the surface itself without penetrating into the surface. Although in both the adiabatic and nonadiabatic case the second sublayer is the second most populated region for the not-scattered $\mathrm{H}$ atoms, the way by which the atoms came to rest here differs for both cases again considerably. For the adiabatic case, the highest contribution was made by coming back from deeper penetration sites whereas in the nonadiabatic case, most of the atoms trapped in the second sublayer came directly to rest here and comparatively few emerged from deeper regions: the mechanism by which $\mathrm{H}$ atoms come to stick on and within the Au-surface is fundamentally different in the adiabatic and nonadiabatic case.

For the scattering trajectories, a similar disparity in behavior can be observed (Tab. 3.10). Although in both adiabatic and nonadiabatic scattering, least $\mathrm{H}$ atoms scatter back after only one collision with a surface atom (single-bounce events), and the greatest number of $\mathrm{H}$ atoms after more than two collisions (multibounce events), the frequencies of the events are very different; if electron hole pair excitation is included, more than $50 \%$ of all scattered $\mathrm{H}$ atoms experience 
Table 3.10.: Outcomes (\%) of scattering for various scattering events resulting from $H$ atom collision with a $\mathrm{Au}(111)$ surface for nonadiabatic and adiabatic simulations. The "Surface"-column refers to trajectories wherein $\mathrm{H}$ atoms scattered from the surface. The Roman numerals refer to the lowest subsurface to which penetration occurred.

\begin{tabular}{lllllllll}
\hline \hline & \multicolumn{3}{c}{ bounce events } & & \multicolumn{5}{c}{ penetrating bounces } \\
& single & double & multi & surface & I & II & III & $>$ III \\
\hline nonadiabatic & 23 & 34 & 43 & 82 & 17 & 1 & 0 & 0 \\
adiabatic & 17 & 25 & 59 & 64 & 23 & 8 & 3 & 2 \\
\hline \hline
\end{tabular}

one or two bounces, while in the electronically adiabatic case only $40 \%$ do. Additionally, in the electronically adiabatic case many more $\mathrm{H}$ atoms (over $1 / 3^{\mathrm{rd}}$ ) penetrate into the surface and up to the lowest sublayers before they resurface and scatter, in the nonadiabatic case less than $20 \%$ penetrate the surface and penetrate no deeper than the second sublayer if they scatter. This behavior is not surprising as the only way $\mathrm{H}$ atoms can lose energy in adiabatic interactions is through highly ineffective collision with the Au atoms. It is therefore to be expected that more $\mathrm{H}$ atoms going through multiple bounce events will scatter back for electronically adiabatic calculations, also including many more that penetrated the slab as it would need them a large number of bounces to lose enough energy to stay within the surface.

The large difference between electronically adiabatic and nonadiabatic simulations is underscored when considering the energy loss behavior: The total energy loss distribution for the electronically adiabatic case is much narrower and the most probable energy loss much smaller than that for the nonadiabatic case (Fig. 3.15(a), inset): the mean energy a hydrogen atom loses in the electronically adiabatically case is a factor of three smaller than when the interaction to electron hole pairs is included (Tab. 3.11). For the adiabatic case, the most probable energy loss is very close to that resulting from a collinear collision between two hard spheres with masses of $\mathrm{H}$ and $\mathrm{Au}: 0.06 \mathrm{eV}, 1.65 \%$ whereas the Baule limit (Eq. 2.58) for the energy loss is $0.07 \mathrm{eV}$

Table 3.11.: Energy loss in \% of incidence energy for various outcomes resulting from $\mathrm{H}$ atom collision with a $\mathrm{Au}(111)$ surface for nonadiabatic and adiabatic simulations. The mean and maximum energy loss are shown for the total ELD and for scattering along $\theta_{\text {out }}=45^{\circ}$ $\phi_{\text {out }}=60^{\circ}([10 \overline{1}])$. The reduced accuracy for the scattering angle reflects the lower signalto-noise ratio of the differential ELD.

\begin{tabular}{lllll}
\hline \hline & Total & \multicolumn{3}{c}{$\theta_{\text {out }}=45^{\circ} \phi_{\text {out }}=60^{\circ}$} \\
conditions & Mean & Peak & Mean & Peak \\
\hline nonadiabtic & 39.2 & 14.0 & 37 & 15 \\
adiabatic & 13.1 & 1.65 & 11 & 1.4 \\
\hline \hline
\end{tabular}




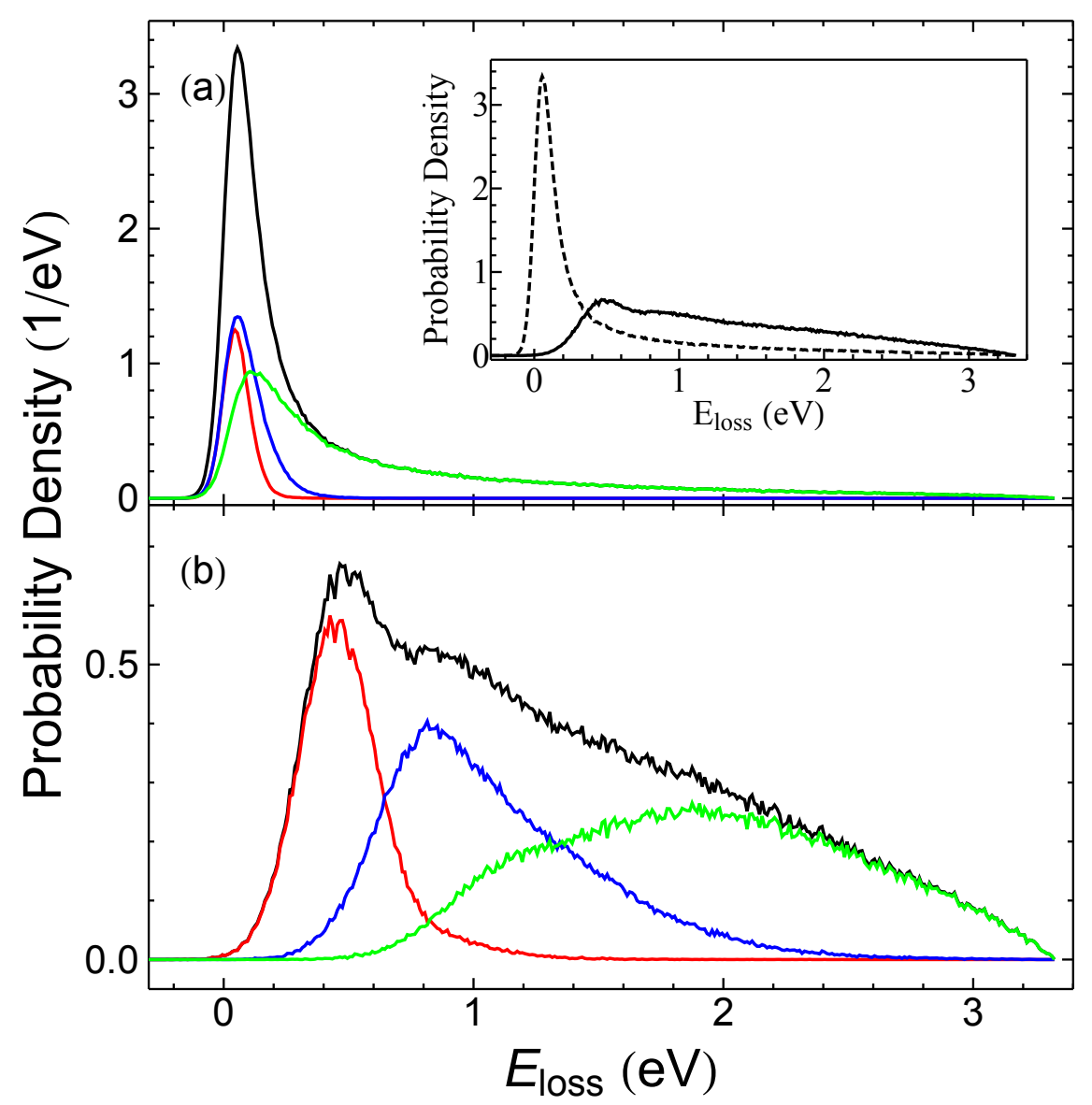

Figure 3.15.: Total energy loss distribution divided into total (black), single bounce (red), doublebounce (blue) and multibounce (green) events. Panel (a) depicts the electronically adiabatic case and panel (b) the nonadiabatic case. The inset in panel (a) shows a comparison between the total energy loss distribution for the adiabatic (dashed line) and nonadiabatic (solid black line)case.

or, $2.00 \%$ ). In the nonadiabatic case, the most probable energy loss is a factor of eight higher energy values.

For the nonadiabatic case, it becomes evident from the total ELD in Fig.3.15(b) that smallest energy losses are occasioned by single-bounce events. The peak of the total energy loss distribution is mainly due to single-bounce collisions with the surface and the fastest part of the $\mathrm{H}$ atom experiencing double-bounce collisions. The double-bounce collisions and multibounce collisions give rise to a shoulder around $1 \mathrm{eV}$ energy loss and the long tail of the total ELD to high energy losses is mostly constituted from multibounce events.

The adiabatic total ELD (Fig. 3.15(a)) does not exhibit as distinct features as the nonadiabtic total ELD, although here, too, the high-energy-loss tail stems from multibounce collision with the surface. Single- and double-bounce collision profiles overlap much more strongly than in the 
nonadiabatic case: the influence of electronic excitation on the translational energy loss of an $\mathrm{H}$ atom is prodigious, the inelasticity increases dramatically with the inclusion of electron hole pair excitation, broadening the entire total energy loss distribution as well as the contribution of the individual bounce events.

Experimentally, the sticking probabilities of $\mathrm{H}$ to $\mathrm{Au}(111)$ have, to my knowledge, not yet been measured. It furthermore appears experimentally difficult to access the total energy loss distribution; much more feasible is the production of differential (angular resolved) energy loss distributions. In a comparison with experiment, it would be good if conditions could be identified where specific scattering energy loss mechanisms could be discerned such as scattering after the collision with one or two surface atoms or scattering after resurfacing.

Figure 3.16 shows the differential energy loss distributions of specular scattering for the electronically adiabatic (left) and nonadiabatic (right) cases. In both cases, the shape of the differential energy loss distribution is again much different, in a manner as has already been observed for the total energy loss distribution. The total angular distributions in the insets show the scattering intensity dependent on the scattering angle. Both in adiabatic and nonadiabatic case, the area of highest scattering probability is in forward scattering direction; but the direction of specular scattering (black circle) is already shifted from the region of maximum scattering intensity. The region of highest scattering intensity finds itself at angles closer to the surface normal. Little intensity is to be seen in back-scattering direction. The shift of the highest scattering intensity from specular angle towards the surface normal is probably caused by the fact that the $\mathrm{H}$ atoms do not impact on a flat surface but a corrugated surface with protrusions above the surface atoms; that there is less scattering in backwards direction is most likely a result of the fact that the incidence energy of the $\mathrm{H}$ atoms is so high that, if they impact close to the a-top site, they are reflected into forward direction. If they impinge lower on the atom, they might be reflected backwards, but presumably at such low angles that they either experience further collisions (which may again fling them forward) or get trapped at the surface.

It is however evident for both cases that under specular scattering (Fig.3.16(a)), the differential energy loss distribution resulting from different bounce events overlap to a very large degree, making it impossible to resolve them individually: in the adiabatic case, the single- and double-bounce events peak at the same energy loss values and show almost a complete overlap, apart from a somewhat broader tail to higher energy losses for the double-bounce events. Energy losses due to multibounce events start to become important at marginally higher energy losses, but also the differential energy loss distribution for the multibounce events peaks at values where there is still a considerable contribution due to single- or double-bounce events. Very high energy losses are made up almost exclusively of multibounce events.

In the nonadiabatic case (Fig. 3.16.(b)), the separation between the differential energy loss distributions due to different bounce events is clearer; the differential energy loss distributions 

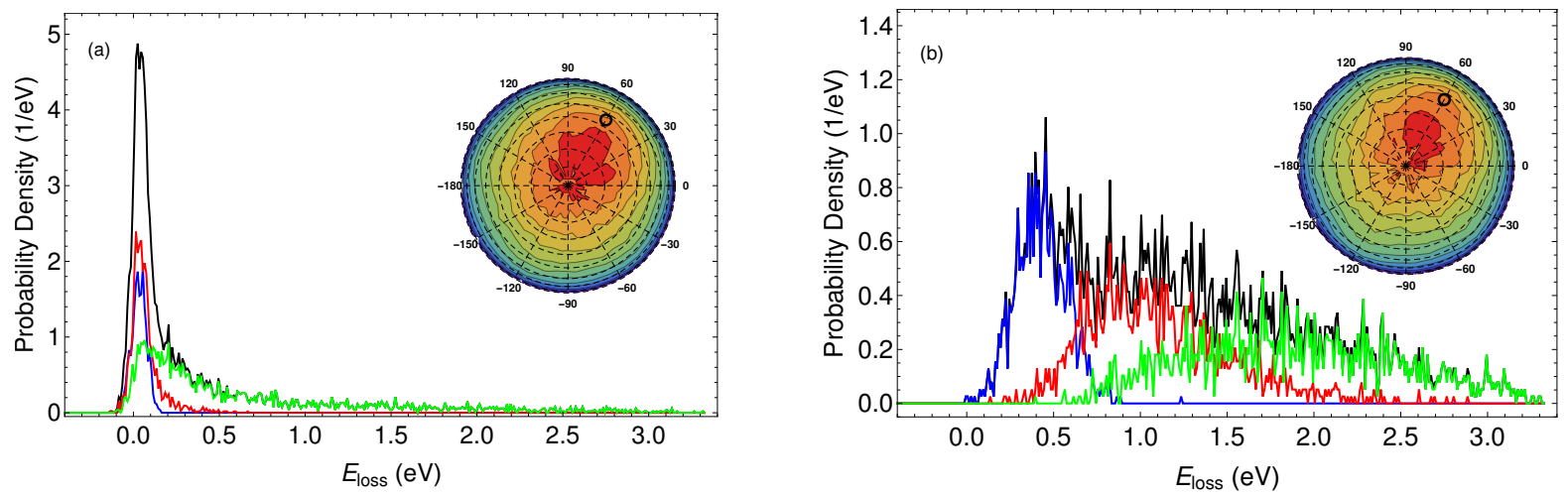

Figure 3.16.: Differential energy loss distribution for specular scattering $\left(\theta_{\text {out }}=45^{\circ}, \phi_{\text {out }}=60^{\circ}\right)$, divided into total (black), single-bounce (blue), double-bounce (red) and multibounce (green) events for the electronically adiabatic (left) and nonadiabatic (right) case. The insets show the total angular distribution. The polar scattering angle is denoted by the distance from the center of the plots starting with $\theta_{\text {out }}=0^{\circ}$ in the middle to $\theta_{\text {out }}=90^{\circ}$ at the outermost rim. $\phi_{\text {out }}$ is indicated explicitly. Red denotes $>92 \%$ in scattering intensity and every contour denotes a drop by $1 / 12$ in intensity so that purple marks the regions of lowest intensity. The black circle indicates the specular angle.

of all three events peak at different energy losses (single-bounce: $\sim 0.4 \mathrm{eV}$, double-bounce: $\sim 0.8 \mathrm{eV}$, multibounce: $\sim 1.9 \mathrm{eV}$ ) and there is little overlap between the single- and the multibounce differential energy loss distributions. However, both differential energy loss distributions for single- and multibounce are much overlapping with the one resulting from double-bounce events. Under the exit angles of specular scattering, the separation in energy loss of different collision events (that is, different energy loss mechanism due to the number of bounces) is impossible. In the following sections, I will therefore consider the different collision mechanisms in detail and try to identify conditions under which a separation of the different energy loss mechanisms might be affected or under which contributions due to different energy loss mechanisms are particularly strong. In this, I will focus on the analysis of the results from the nonadiabatic simulations, for, as will be shown in section 4 , they reproduce the experimental energy loss behavior which simulations without electron hole pair excitation do not. I will therefore only give the most general overview over the behavior to be expected from adiabatic scattering.

\section{Single-Bounce Events}

The simplest energy loss mechanism for scattering with a surface that can be imagined is a single collision with an $\mathrm{Au}$ atom with subsequent scattering of the $\mathrm{H}$ atom from the surface.

Figure 3.17 shows the angular distribution for single bounce events (right) and the impact sites within the primitive cell (left). For both adiabatic and nonadiabtic simulations, the behavior is very similar with regards to the impact site which is close to the a-top site. The impact sites 


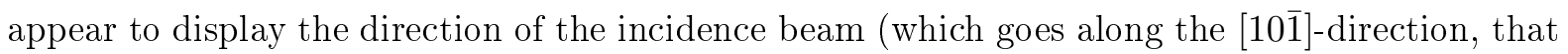
is to say along the dashed line from $\phi=-120^{\circ}$ to $\phi=60^{\circ}$ in the angular distribution plots). Furthermore, the impact sites barely extend behind the middle of the primitive cell as if the incident atoms undergoing single-bounce collisions only hit the ascent side of Au atoms (the side of the Au atoms directed towards the beam). In scattering, there are two major maxima both adiabatic and nonadiabatic simulations have roughly in common, one where scattering in azimuthal direction is along the forward scattering direction at $\phi_{\text {out }} \approx 60^{\circ}$ and in polar direction between $\theta_{\text {out }} \approx 20-50^{\circ}$, the other at $\phi_{\text {out }} \approx 85^{\circ}$ and $\theta_{\text {out }} \approx 20-40^{\circ}$, for the adiabatic case and $\theta_{\text {out }} \approx 15-30^{\circ}$ for the nonadiabatic case. In the nonadiabatic simulation, there is an additional other small maximum extending from the surface normal along $\approx 115^{\circ}$ to $\theta_{\text {out }} \approx 25^{\circ}$. For both the adiabatic and the nonadiabatic case, there is an additional a weak maximum in backscattering direction at $\phi_{\text {out }} \approx 120^{\circ}$. The six-fold symmetry of the surface is hinted at in very two very weak features at $\phi_{\text {out }} \approx-55$ and $\phi_{\text {out }} \approx 170$.

To see how single-bounce events influence the structure of the differential energy loss distribution, and if conditions can be identified where features in the differential energy loss distribution due to single-bounce events may be experimentally distinguishable from the other bounce events, I have investigated the differential energy loss distribution at the maxima in intensity of the angular distribution for single bounce events at $\theta_{\text {out }}=25^{\circ}$ and $\phi_{\text {out }}=90^{\circ}$ (Fig. 3.18)(a)), at $\theta_{\text {out }}=15^{\circ}$ and $\phi_{\text {out }}=115^{\circ}$ (Fig. 3.18(b)) and at $\theta_{\text {out }}=35^{\circ}$ and $\phi_{\text {out }}=60^{\circ}$ (Fig. 3.18(c)). The first and second of these exiting angles also correspond to cuttings of the region of maximum intensity of the angular distribution (see Fig. 3.14).
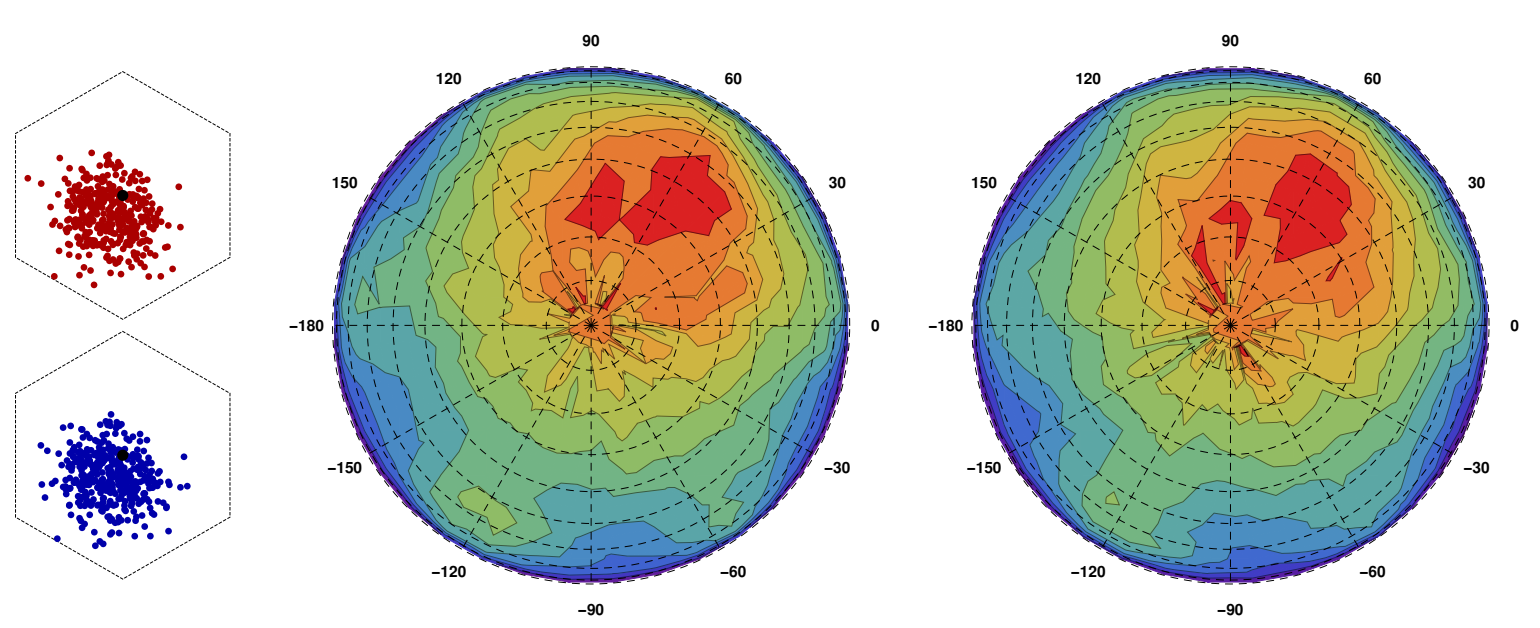

Figure 3.17.: Left: Impact sites within the primitive cell for electronically adiabatic (top, dark red) and nonadiabatic (bottom) case for $\mathrm{H}$ atoms undergoing a single bounce. Right: angular distribution for single bounce events in scattering collisions for electronically adiabatic (left) and nonadiabatic (right) simulations. For details see caption of Fig. 3.16 

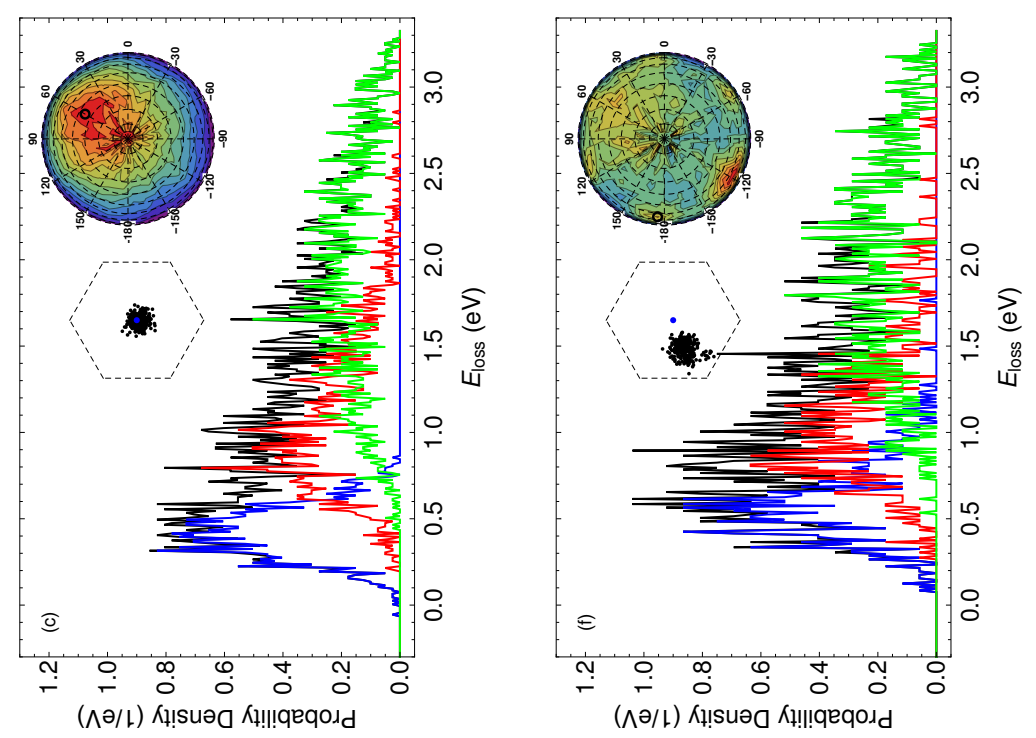

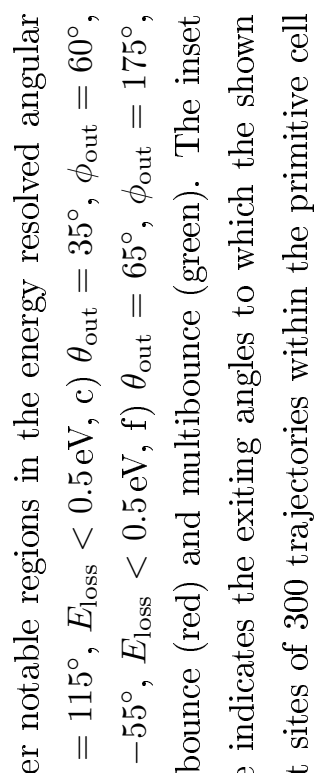
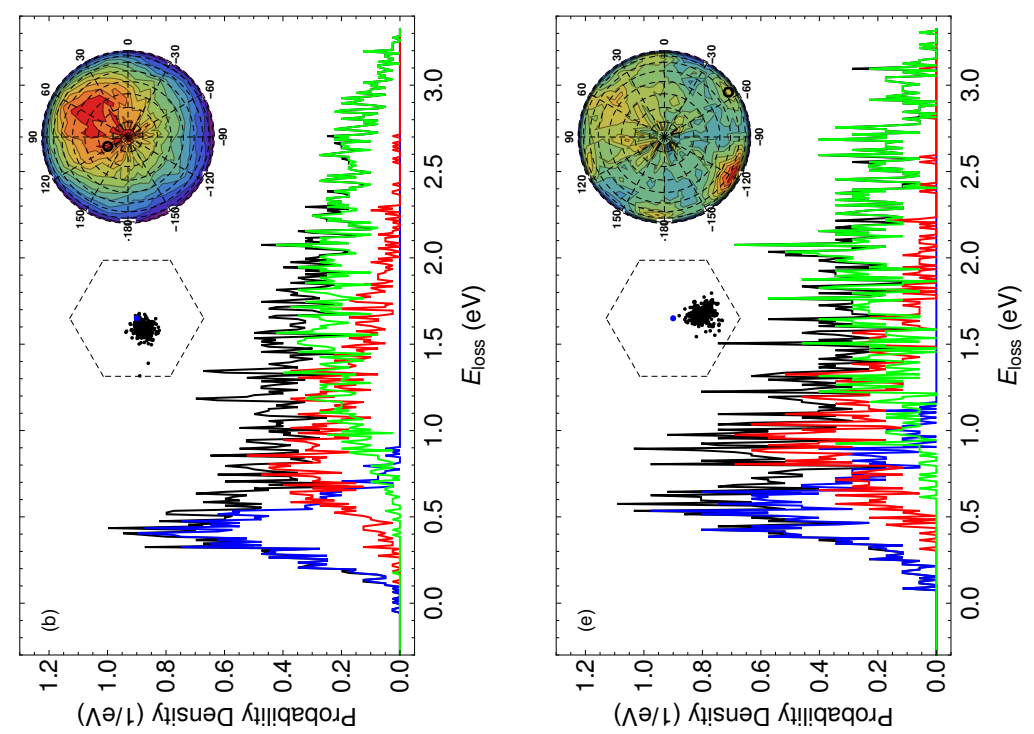

离

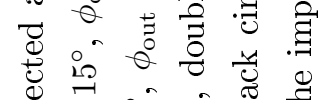

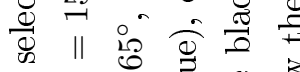
\& ฮี 2 苛

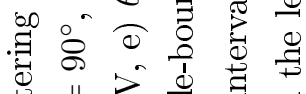

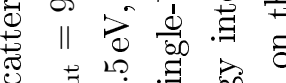
跑

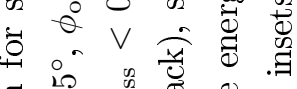

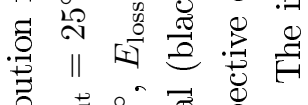

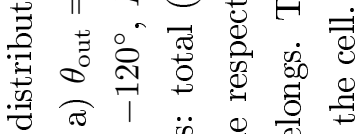
會
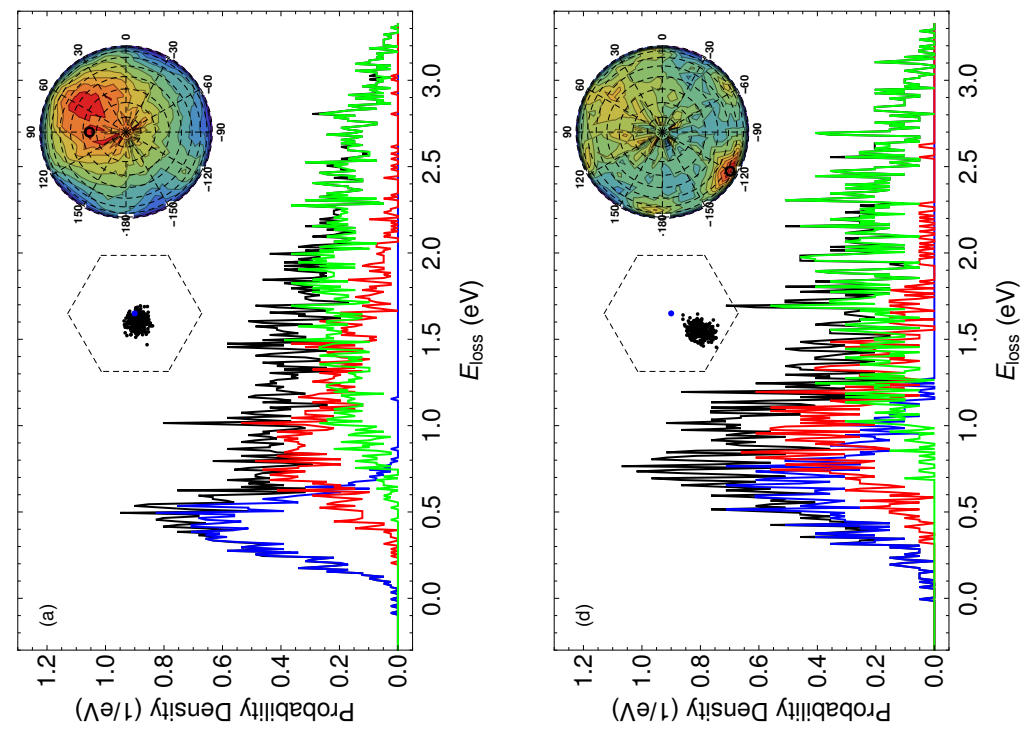

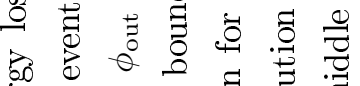
氙过 종

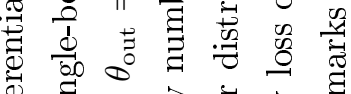

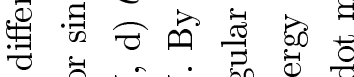
के .

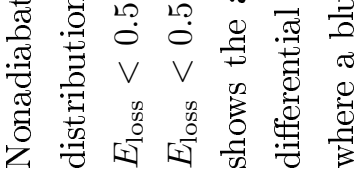


The single-bounce events can furthermore be separated by the amount of energy lost into two different categories. Firstly, $\mathrm{H}$ atoms that lose little energy (below $0.5 \mathrm{eV}$ ) and scatter very close to the top site mostly into forward direction (see insets in Fig. 3.18(b) and (c)). Their maxima in scattering intensity are identical to those of the total angular distribution for single-bounce events as can be seen if the total angular distribution for single-bounce events (Fig. 3.18(a), right inset) and the angular distribution of scattering for $E_{\text {loss }}<0.5 \mathrm{eV}$ (see Fig. 3.18 (b)-(c)) are compared.

Secondly, single-bounce events that exhibit higher energy losses (above $0.5 \mathrm{eV}$ ). In this case, the maxima in forward scattering disappear and the back-scattering peak become more pronounced (Fig. $3.18(\mathrm{~d})-(\mathrm{f})$, right inset) and the sideways back-scattering peaks at $\phi_{\text {out }}=-55^{\circ}$ and $\phi_{\text {out }}=$ $175^{\circ}$ become clearer. The impact sites for these collisions also show a very distinct positioning: they are much more shifted from the a-top site and gathered towards the bridge sites which corresponds roughly with the direction under which the $\mathrm{H}$ atoms return. Interestingly, this return direction is not directly align with the hollow sites, but by $\sim 5^{\circ}$ shifted from the exact direction where the neighboring Au atoms lie. The slightly broader angle of scattering is most likely due to the fact that, if the $\mathrm{H}$ atoms were scattered exactly towards the Au atoms, they would experience another bounce. It allows the scattered $\mathrm{H}$ atom to fly along the groove created by the hollow sites and in this manner escape another collision before they can leave the surface; if they were scattered by a larger angle into the direction of a hollow site, the (see Fig $2.1(\mathrm{~b})$ ) scattering would soon bring them into collision with a neighboring Au atom. Flying along the groove of the hollow sites also allows the $\mathrm{H}$ atoms to escape the surface at a rather low polar scattering angle, but by that increasing the time they spend inside the background electron density and thereby increasing their energy loss, explaining why the maxima in intensity caused by these processes become much more evident when low-energy scattering is excluded. The intensity of these scattering events is by roughly a factor of two smaller than the intensity of scattering in forward direction leading to very noisy differential energy loss distributions.

Unfortunately, the differential energy loss distributions of the single-bounce events in forward scattering direction are severely overlapped by the differential energy loss distributions of the double-bounce-events. The differential energy loss distributions of the single-bounce events in forward scattering direction are narrow and have a high intensity compared to the rest of the differential energy loss distribution (see Fig. 3.18 and Fig. 3.19 (a), dark yellow, blue and purple). The differential energy loss distributions in back-scattering direction contain weak single-bounce contributions compared to the contributions from the other bounce events and the differential energy loss distribution of single-bounces overlaps even stronger with that of the other bounce events. Furthermore, they are much broader than the single-bounce components in forward scattering direction (compare green, black and red for scattering in backward direction with dark yellow, blue and purple in Fig. 3.19 (a)): A clear separation of the single-bounce 

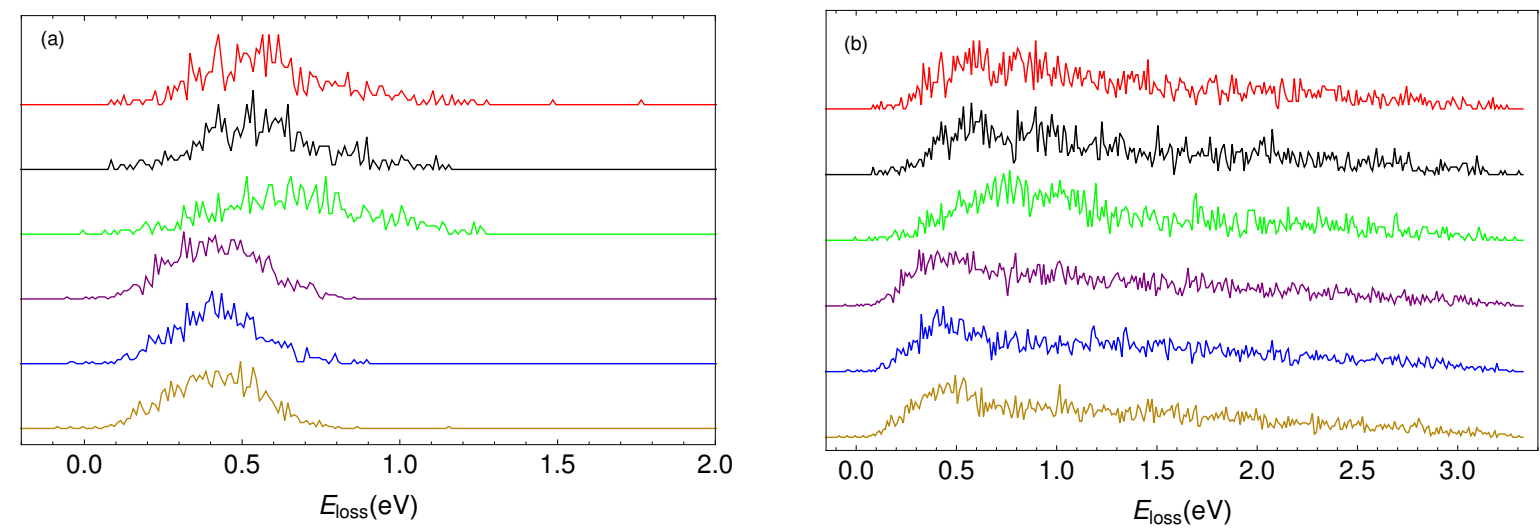

Figure 3.19.: Differential energy loss distribution for exit angles $\theta_{\text {out }}=25^{\circ}, \phi_{\text {out }}=90^{\circ}$ (dark yellow), $\theta_{\text {out }}=15^{\circ}, \phi_{\text {out }}=115^{\circ}$ (blue), $\theta_{\text {out }}=35^{\circ}, \phi_{\text {out }}=60^{\circ}$ (purple), $\theta_{\text {out }}=65^{\circ}, \phi_{\text {out }}=-120^{\circ}$ (green), $\theta_{\text {out }}=65^{\circ}, \phi_{\text {out }}=-55^{\circ}$ (black), $\theta_{\text {out }}=65^{\circ}, \phi_{\text {out }}=175^{\circ}$ (red): (a) single-bounce and (b) total.

differential energy loss distribution from the other bounce events appears impossible under the present conditions. However, as a lowering of the temperature causes the surface to be less corrugated, it appears possible that at lower temperatures a clearer separation of the bounce events could be effected, at least for the differential energy loss distribution of forward scattering (see Section 3.3.2.

On the other hand, the overall form of the differential energy loss distributions in forward scattering direction (see also Fig. 3.19 (b), dark yellow, blue and purple) differs from those in backward scattering direction, due to the decline of the intensity of the single-bounce events differential energy loss distribution and the rise of that of the double-bounce events. Even thought the differential energy loss distributions are noisy and peak positions therefore difficult to assign, the peaks of the differential ELD for backscattering appear to be shifted by $\sim 100 \mathrm{meV}$ compared to those for forward scattering. This shift in energy loss and perhaps the difference in shape could conceivably be observable experimentally and explained with the findings presented here.

\section{Double-bounces Events}

The second distinct energy loss mechanism for scattering from a surface that can be imagined as a double collision with two $\mathrm{Au}$ atoms and subsequent scattering of the $\mathrm{H}$ atom from the surface. The left insets in Figure 3.20 show the first impact sites of double-bounce trajectories, which for the adiabatic and nonadiabatic case are similar notwithstanding that the maximum of intensity in the nonadiabatic case extends from closer to the surface normal and appears less sharply defined than in the adiabatic case. In comparison to the first impact site of single-bounce trajectories (Fig. 3.17) the first impact site for double-bounce events is more shifted from the 

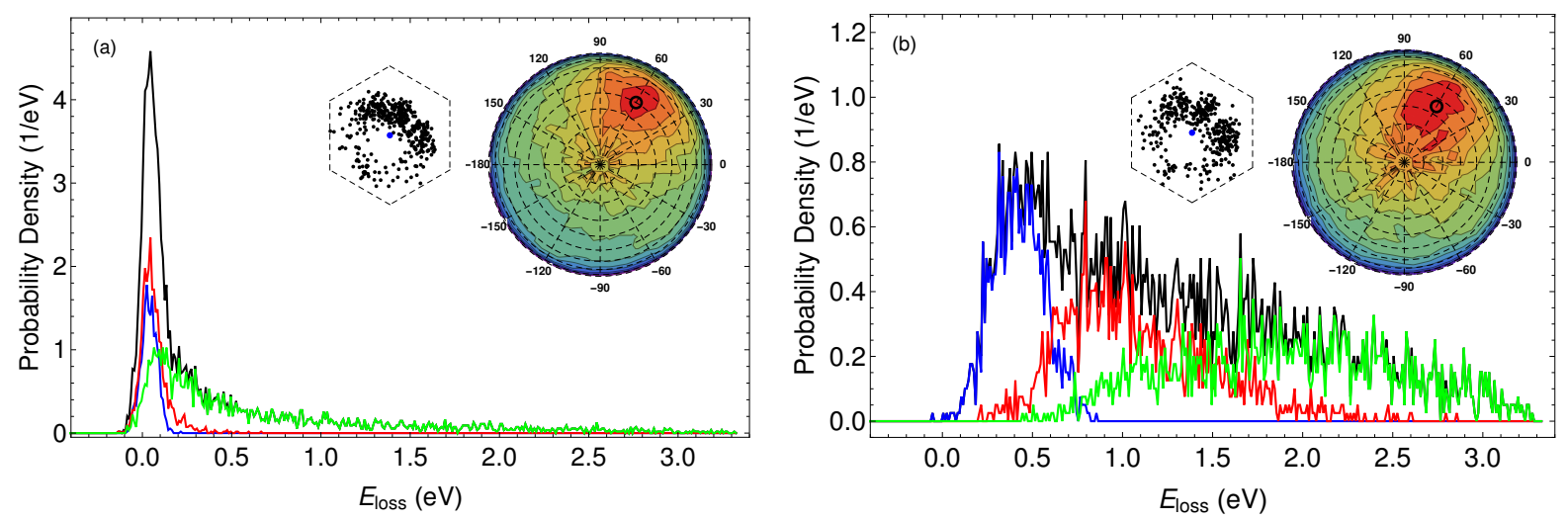

Figure 3.20.: Differential energy loss distribution for the maximum in intensity of double-bounce scattering events for electronically adiabatic $\left((\mathrm{a}), \theta_{\text {out }}=40, \phi_{\text {out }}=60\right)$ and nonadiabatic $\left((\mathrm{b}), \theta_{\text {out }}=35, \phi_{\text {out }}=60\right)$ case. The left inset shows the first impact site within the primitive cell for 400 trajectories respectively, the right inset the total angular distribution for double-bounce events in scattering collisions where the black circle marks the scattering conditions whose differential energy loss distribution is shown.

a-top site towards the hollow and bridge sites. Differently from the collision that lead to singlebounce events, the first impact sites leading to double-bounce events appear to be distributed around the middle of the atom, not solely arranged in the rough direction of the incidence beam. The beam-averted incidence sites of the Au atoms (the far side of the primitive cell) are closer toward the a-top site than the ones on the site facing the $\mathrm{H}$ atom beam.

The maximum in intensity in scattering as can be seen in the total angular distribution for the adiabatic (Fig.3.20(a), right inset) and nonadiabatic (ig. 3.20(b), inset, red area) case is as for the case of single-bounce scattering along the forward scattering direction. The area of highest scattering intensity spans from $\theta_{\text {out }} \approx 20-55^{\circ}$ and $\phi_{\text {out }} \approx 25-95^{\circ}$ and therefore occupies a very similar area as the maximum in scattering intensity of the single-bounce events. Scattering in backwards direction is weaker than for the single-bounce case. In the adiabatic case, the area of highest intensity is between $\theta_{\text {out }} \approx 30-55^{\circ}$ and $\phi_{\text {out }} \approx 45-75^{\circ}$ and there is very little scattering in the backwards direction.

Both differential energy loss distributions for the maximum in scattering intensity for electronically adiabatic and nonadiabatic case (Fig. 3.20 ) show much overlap of the different bounce events. The shape of the differential energy loss distribution caused by adiabatic scattering and by nonadiabatic scattering differs again significantly, although due to $\mathrm{H}$ atoms hitting similar impact sites.

The double-bounces for scattering including electron hole pair excitation can be further classified after the distance in which their second bounce happened (Fig. 3.21): from a histogram of the inter-bounce distances, it can be concluded that $58 \%$ of the double-bounces occur with the nearest neighbor Au atoms with no preference to scattering direction (black, Fig 3.21), $8 \%$ occur 
with the next nearest neighbor Au atoms (blue); these collisions occur by flying over the hollow

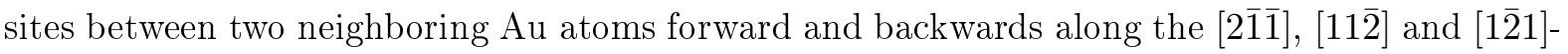
directions. According to these directions, one would expect to see a $\mathrm{C}_{6}$ symmetry in the angular distribution (Fig.3.21(a), second inset), but most of these collisions appear to lead to nearly specular scattering $\left(\theta_{\text {out }}=45^{\circ}, \phi_{\text {out }}=55^{\circ}\right)$, it may safely be assumed, caused by the direction taken up after the second bounce. $1 \%$ of the double-bounces occurs with the next-next nearest neighbors; the number of these events is presumably this low because most of the $\mathrm{H}$ atoms that scatter into the direction of the next-next nearest neighbors collide with the nearest neighbors (green, see Fig. 3.21). No angular distribution is presented for it, the probability for these events being so low that not enough $\mathrm{H}$ atoms are scattered for an angular distribution. $16 \%$ of all double-bounces fly as far as the $3^{\text {rd }}$ nearest neighbors. As can be seen from Fig 3.21(a) (red), these double-bounces occur into all directions with an emphasis of scattering in the forward direction and closer to the surface normal. The rest of the double-bounce events (17\%) happen with $\mathrm{Au}$ atoms even further away (up to $218 \AA$ ) and also show a preference of scattering with a $\theta_{\text {out }}$ closer to the surface normal and into forward direction.

The total energy loss distributions resulting from the double-bounce events with different inter-bounce distance is shown in Fig 3.21 (c). Predictably, those double-bounce events that fly longer distances lead to higher energy losses. If the maximum in an angular distribution is mostly due to one such distribution, it could be expected that they lead to a significant change in the shape in the differential energy loss distribution that might be observed in experiment. To test this, I therefore extracted differential energy loss distributions (Fig. 3.22(a)-(d)) for such exit angles under which the distance-resolved double-bounce events appeared to exhibit maxima in intensity (Fig. 3.21(a), black circles in insets). While the shape of the differential energy loss distribution of the double-bounce events changes for the chosen exit conditions (see Fig. 3.22(e)), this does not effect the shape of the total differential energy loss distribution greatly (Fig. $3.22(f)$ ). It seems that the shape of the differential energy loss distribution is more influenced by the ratio of intensity of the differential energy loss distributions of the singlebounce events to the double-bounce events to the multibounce events. This means that while the double-bounce events show an astounding variety in the distance they cover between the first and second bounce, the discrimination between these bounce behaviors in experiment appears impossible: scattering with inter-bounce distances that take preferential directions on the surface is so weak or directed into similar directions as preferred by single-bounce events that it makes no impact on the complete differential energy loss distributions.

Figure 3.23 shows the double-bounce events resolved after the traveling distance for the adiabatic case. While the percentage of atoms colliding with the neighboring atoms (58\%), the next neighboring atoms $(8 \%)$, the next-next $(<1 \%)$ are the same as in the nonadiabatic case, a much lower number of $12 \%$ collides with the $3 \times$ next-nearest neighbors, and the number of 

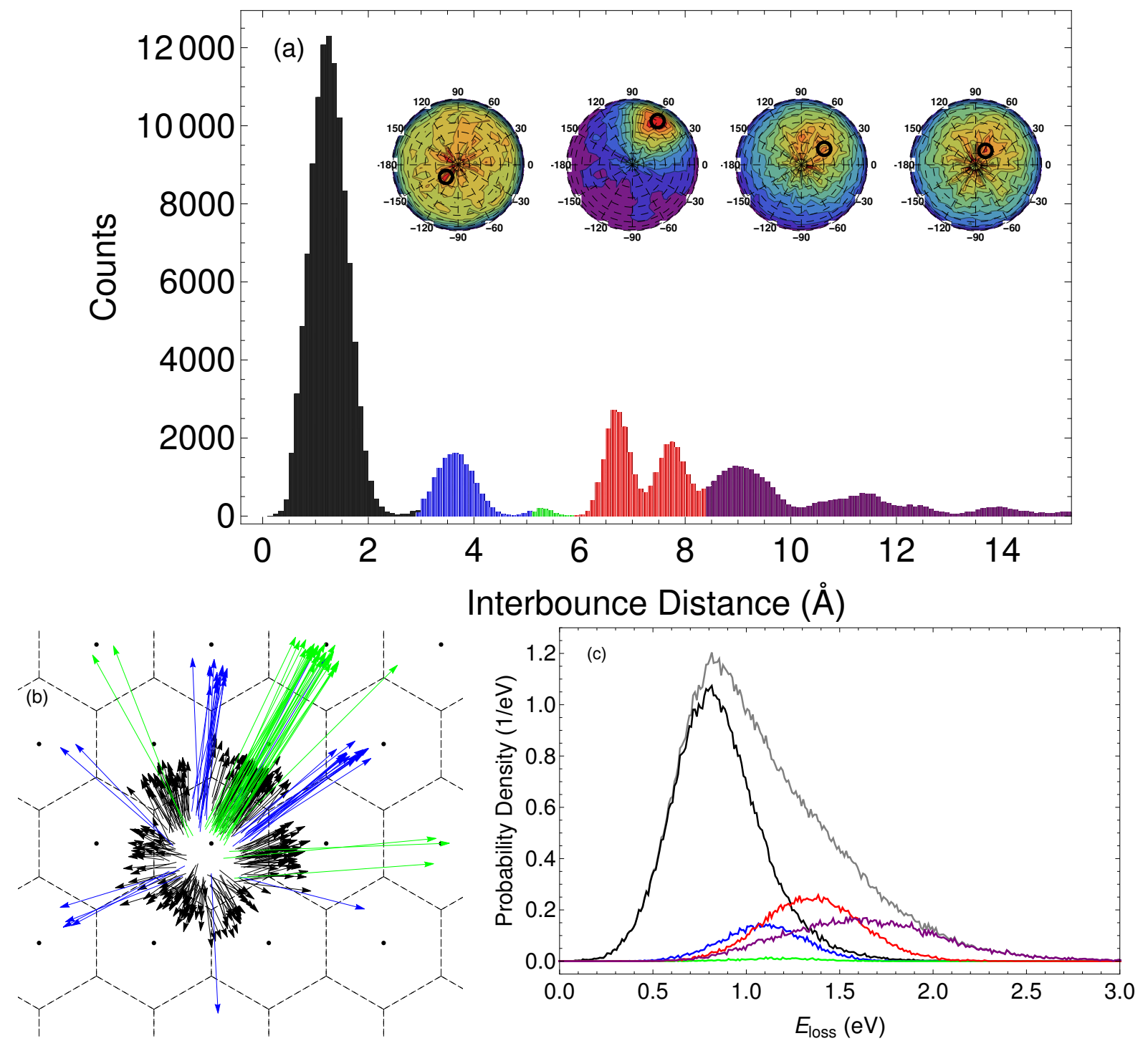

Figure 3.21.: Analysis of double-bounce trajectories with respect to their second impact site for the electronically nonadiabatic case. (a) A histogram of the distance traveled between the bounces. The colors mark the collisions with different neighboring Au atoms: black: nearest neighbor, blue next-nearest neighbor, green: next-next-nearest neighbor, red: $3 \times$ next-nearest neighbor, purple: all other neighbors. The inset in (a) is the angular distribution from left to right for the events marked black, blue, red and purple. The black circle in it marks the scattering region from which the differential energy loss distribution in Fig 3.22, a)-(d) were drawn. (b) Vectors connecting the first and the second bounce site. The number of arrows is not representative for the frequency of the events. The influence of the surface structure on the distance between the bounce sites is clearly visible. (c) shows the nonadiabatic total energy loss spectrum for the double-bounce events with the contribution separated after different inter-bounce distance. 

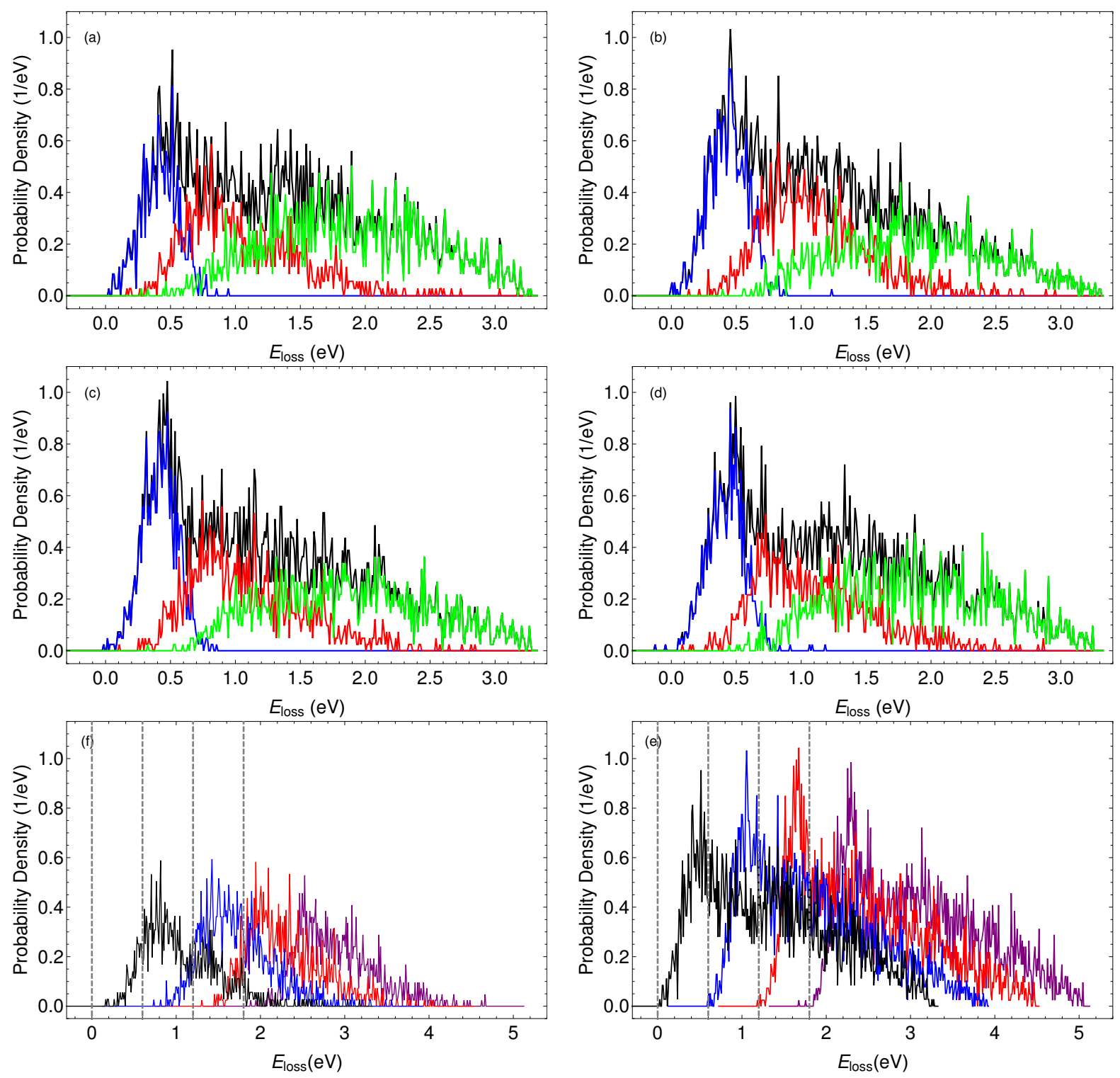

Figure 3.22.: ((a)-(d)) Differential energy loss distributions for exit angles (a) $\theta_{\text {out }}=15^{\circ}, \phi_{\text {out }}=-135^{\circ}$, (b) $\theta_{\text {out }}=50^{\circ}, \phi_{\text {out }}=60^{\circ}$, (c) $\theta_{\text {out }}=25^{\circ}, \phi_{\text {out }}=35^{\circ}$, (d) $\theta_{\text {out }}=15^{\circ}, \phi_{\text {out }}=55^{\circ}$ according to the maxima in intensity of the angular distributions in Fig.3.21(a). The differential energy loss distributions are subdivided into: total (black), single-bounce (blue), double-bounce (red) and multibounces (green). (e) The differential energy loss distribution for double-bounce events (red curves (a)-(d)) according to the color code applied in Fig. 3.21. (f) The complete differential energy loss distribution (black curves in (a)-(d)) according to the color code applied in Fig. 3.21. The distributions are shifted by $0.6 \mathrm{eV}$ to one another and the dashed grey lines mark the zero point in energy loss for each of them.

far-distant collisions is increased to $22 \%$. Also, the scattering pattern changes slightly if the intensity distribution of the adiabatic case (insets in Fig. 3.23 are compared with those of the 


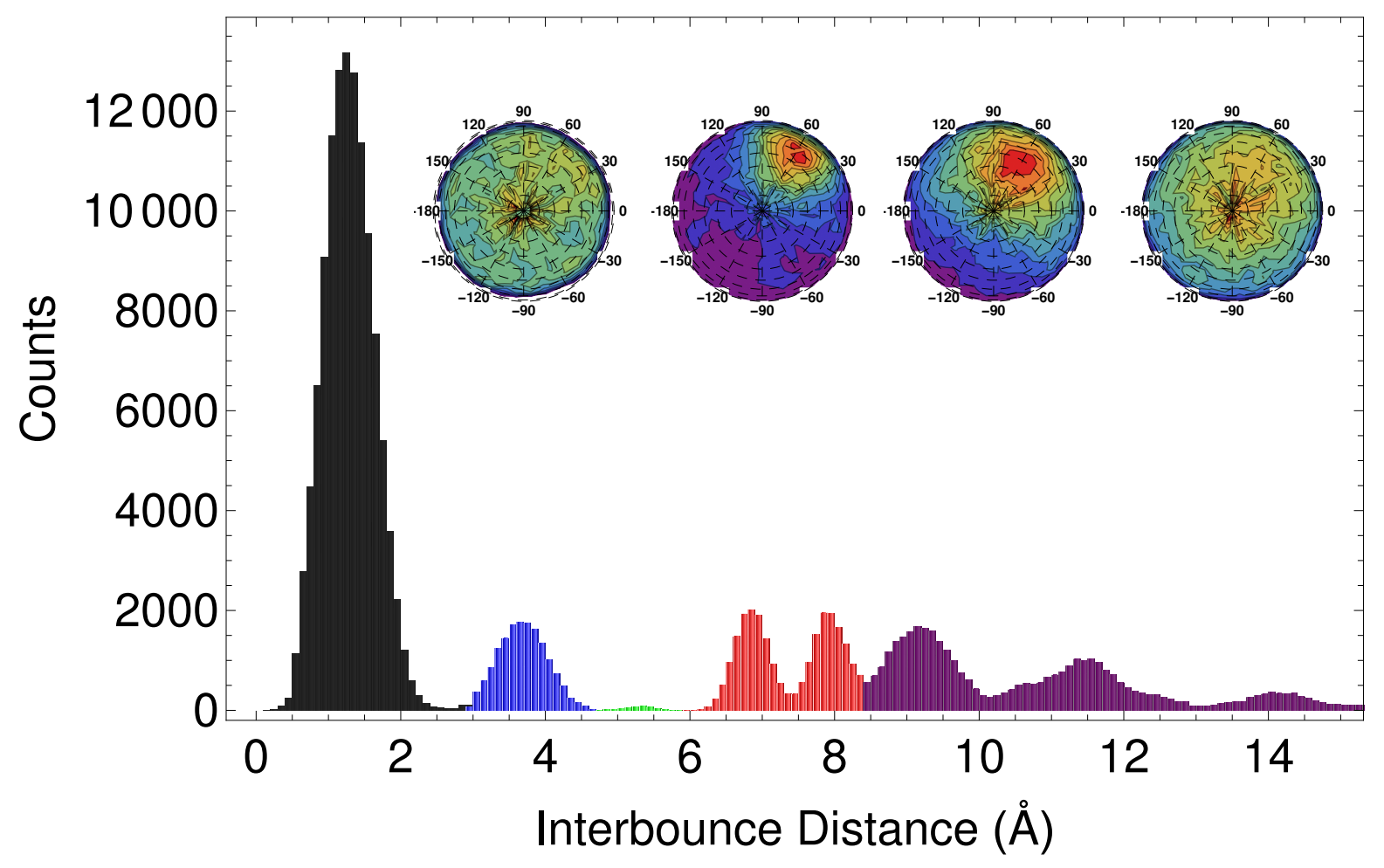

Figure 3.23.: Histogram of the distance traveled between the bounces for adiabatic double-bounce trajectories. The colors mark the collisions with different neighboring Au atoms: nearest neighbor (black), next-nearest neighbor (blue), next-next-nearest neighbor (green), $3 \times$ next-nearest neighbor (red), all other neighbors (purple). The insets are the angular distribution from left to right for the events marked black, blue, red and purple.

nonadiabatic case (insets in Fig. 3.21 (a)): while the angular distribution are similar for the scattering event with the nearest and next-nearest neighbors, they differ significantly for scattering events with the $3 \times$ next-nearest neighbors (third insets), showing a clear directional preference for forward scattering in the adiabatic case and a lesser preference in the nonadiabatic case. This could be explained with the observation that a significantly larger percentage of trajectories in the nonadiabatic case fall into this category than in the adiabatic case which in turn could be due to the background electronic density influencing the scattered atoms in such a manner that they are brought to collide with closer atoms by being slowed down or that atoms that fly too far lose too much of their energy to leave the surface. The farthest distance $\mathrm{H}$ atoms fly in the adiabatic case is over $200 \AA$, in the nonadiabatic case less than $160 \AA$.

Again, it becomes evident that the dynamics in the electronically adiabatic and nonadiabatic case differ significantly. 

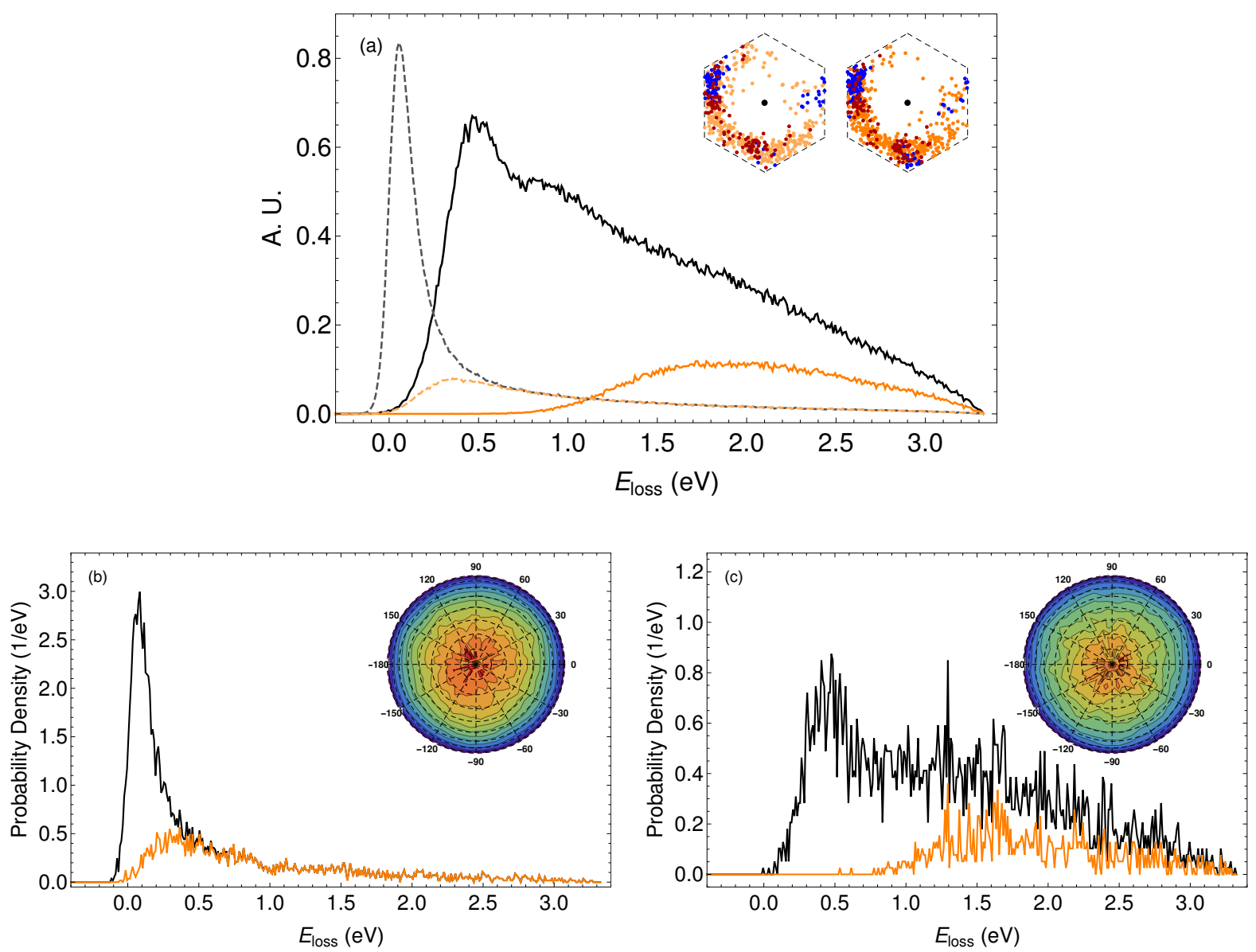

Figure 3.24.: (a) Total energy loss distribution of all scattering trajectories for the adiabatic (dashed, gray) and nonadiabatic (black) case, and for the scattering trajectories that penetrate the surface for the electronically adiabatic case (dashed, orange) and the nonadiabatic case (orange) with their respective first impact sites within the primitive cell (insets, orange: all bounce events (400 trajectories), blue: single-bounce trajectories (all trajectories), dark-red: double-bounce (100 trajectories)). The differential energy loss distribution at $\theta_{\text {out }}=0.0^{\circ}$, corresponding to the maximum of intensity exhibited in the total angular distribution of penetrating, scattering trajectories in (b) adiabatic and in (c) nonadiabatic cases. The differential energy loss distribution of the penetrating (orange) events are shown.

\section{Scattering after Penetration of the Surface Layer}

The last significant mechanism that can be imagined involves $\mathrm{H}$ atoms that penetrate the surface (that is, pass below $0.0 \AA$ ) and scatter then. The question is raised if they behave differently causing remarkable features which could be observed experimentally. Figure 3.24(a) shows the total energy loss distribution for the scattering trajectories that penetrate the surface. In the electronically adiabatic case, these trajectories make out most of the tail of the total energy loss distribution (compare black and orange dashed curve) and form a very broad distribution that is 
much wider than that for the non-penetratively scattered atoms. This behavior is in agreement with the observations of Kroes and coworkers [3, 4] on the behavior of penetrating trajectories for $\mathrm{H}$ on $\mathrm{Au}(111)$ but different incidence conditions $\left(E_{\text {inc }}=5.0 \mathrm{eV}, \theta_{\text {in }}=15^{\circ}, \phi_{\text {in }}=90^{\circ}\right.$ and $\left.\theta_{\text {in }}=60^{\circ}, \phi_{\text {in }}=60^{\circ}\right)$. In the nonadiabatice case, the penetrating trajectories account for some of the high energy losses, they do not account for all of them (solid black and orange lines). Indeed, the contribution of penetrating trajectories peaks at about $E_{\text {loss }} \approx 2 \mathrm{eV}$ and drops again towards very high energy losses. The total angular distributions (inset Fig. 3.24(b) and (c)) show that the highest intensity for penetrating trajectories is to be expected close to the surface normal. The broadness of the angular distribution of penetrative (and overall) scattering is in agreement with the observations of Kroes and coworkers [3, 4, although they observed no preferential scattering along the surface normal for gold. This is most likely due to the low number of trajectories in their simulations using AIMD, rather than the different incidence conditions or methods. The differential energy loss distributions for normal exit angle for the adiabatic and nonadiabatic case are shown in 3.24 (b) and 3.24 (c), respectively. The difference between the adiabatic and nonadiabatic energy loss distributions are grave as remarked previously, even with respect to the penetrating trajectories: in the adiabatic case, the penetrating trajectories (orange) shape the entire tail of the energy loss distribution and completely account for its form. In the nonadiabatic case, the penetrating trajectories make a contribution to the tail, but do not modify it in any manner that could be expected to be observed experimentally. Furthermore, I was unable to identify any scattering condition where penetrating trajectories do not contribute, neither for the adiabatic, nor for the nonadiabatic case.

As can be seen from the insets in Fig.3.24(a) (points marked in blue), there are $\mathrm{H}$ atoms undergoing penetrating and single-bounce events which hit the surface precisely at the $h c p$ hollow sites. Both for the adiabatic and nonadiabatic case, their contribution is far below $1 \%$ of the penetrating trajectories $(0.1 \%$ in the nonadiabatic and $0.03 \%$ in the adiabatic case). They penetrate no deeper than the first subsurface layer, but have no clear preference of scattering direction. Double-bounce trajectories make up $2 \%$ and $3 \%$ of the penetrating trajectories in the electronically adiabatic and nonadiabatic case, respectively. They enter the surface by the hollow and bridge sites (Fig. 3.24(a), inset, dark read) and preferably return close to the surface normal. The low probability of both the penetrating single and double-bounce events makes their experimental detection very implausible.

\section{Further investigations}

Having not been able to find a clear separation between the bounce events by analysis of scattering behavior, I tried to identify scattering directions where the greatest separation between bounce events in energy loss could be observed. For that, I determined the nonadiabatic differential energy loss distribution in steps of $5^{\circ}$ in $\theta_{\text {out }}$ and $\phi_{\text {out }}$ and noted the highest energy 

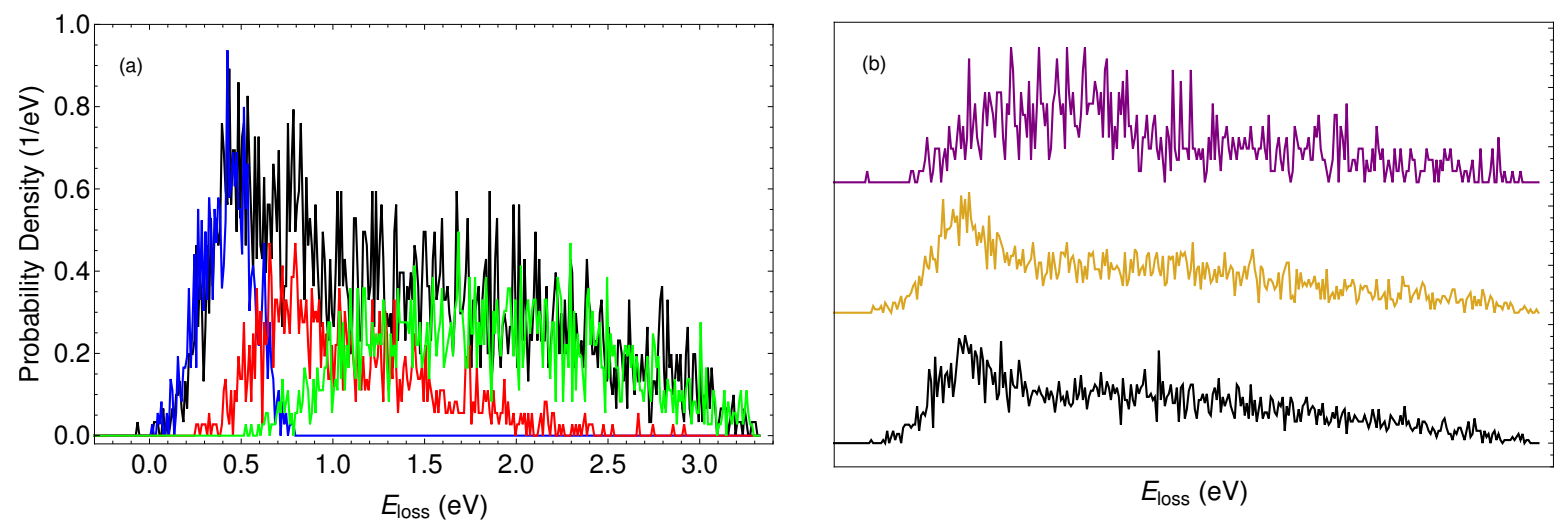

Figure 3.25.: (a) Differential energy loss distribution (black) with the farthest separation between double (red) and single bounce (blue) events at $\theta_{\text {out }}=15^{\circ}$ and $\phi_{\text {out }}=-35^{\circ}$ (green: multibounce events). (b) Comparison between energy distribution of farthest separation between differential energy loss distribution of single and double-bounce (black), strongest single bounce (yellow, $\theta_{\text {out }}=35^{\circ}$ and $\phi_{\text {out }}=10^{\circ}$ ) and strongest double-bounce (purple, $\theta_{\text {out }}=75^{\circ}$ and $\left.\phi_{\text {out }}=-25^{\circ}\right)$ contributions.

loss exhibited by the differential energy loss distribution of the single-bounce events and the lowest of the double-bounces; if single- and double-bounce differential energy loss distribution did not overlap, the difference between those two would have been smaller than zero. The lowest overlap between the differential energy loss distributions of double- and single-bounce is at the exit angles $\theta_{\text {out }}=15^{\circ}$ and $\phi_{\text {out }}=-35^{\circ}$, however, they still overlap over a region of $0.53 \mathrm{eV}$ (Fig. 3.25, (a) and (b), black). Therefore, I decided to find differential energy loss distributions under such exit angles where the differential energy loss distribution of the single-bounce events peaks the highest compared to the maximum of the differential energy loss distributions caused by double-bounce events (yellow, Fig. 3.25 (b)) or where double-bounce events have a very significant contribution (purple, Fig. $3.25(\mathrm{~b})$ ).

All three selected conditions have differently shaped differential energy loss distributions. While the difference between the differential energy loss distribution of the lowest overlap and that of the most intense single-bounce contribution (black and yellow) are only very slight in that the first is somewhat flatter than the second, the differential energy loss distribution of the most intense double-bounce contribution (purple) is considerably broader than the other two, its shape much rounder and its peak appears to be shifted to higher energy losses. It remains to hope that such a difference could also be resolved experimentally. Furthermore, a lowering of the surface temperature should diminish the broadening of the peaks of the different bounce events due to a less corrugated surface at lower temperatures and thereby perhaps making it possible to separate bounces for (a). This influence shall be reviewed in section 3.3.2. 


\section{Angular Distribution}

Another distribution that can conceivably be compared to experiment is the angular distribution in $\theta_{\text {out }}$ at fixed azimuthal angles $\phi_{\text {out }}$. Figure 3.26 shows a comparison of the electronically

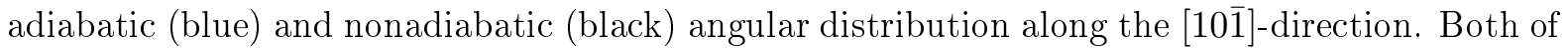
them assume roughly a cosine shape, but the angular distribution of the electronically adiabatic case peaks at $\theta_{\text {out }} \approx 20^{\circ}$ while that of the nonadiabatic case peaks over a wide area between $\theta_{\text {out }} \approx 5--30^{\circ}$.

I have compared the azimuthal angular distribution along the $[10 \overline{1}]\left(\phi_{\text {out }}=60^{\circ}\right.$ or $\phi_{\text {out }}=$ $\left.-120^{\circ}\right),[11 \overline{2}]\left(\phi_{\text {out }}=90^{\circ}\right.$ or $\left.\phi_{\text {out }}=-90^{\circ}\right),[01 \overline{1}]\left(\phi_{\text {out }}=120^{\circ}\right.$ or $\left.\phi_{\text {out }}=-60^{\circ}\right),[1 \overline{1} 0]\left(\phi_{\text {out }}=0^{\circ}\right.$ or $\left.\phi_{\text {out }}=-180^{\circ}\right),[1 \overline{2} 1]\left(\phi_{\text {out }}=-30^{\circ}\right.$ or $\left.\phi_{\text {out }}=150^{\circ}\right)$ and $[2 \overline{1} \overline{1}]\left(\phi_{\text {out }}=30^{\circ}\right.$ or $\left.\phi_{\text {out }}=-150^{\circ}\right)$ surface directions. For this, I define scattering between the azimuth angles $\phi_{\text {out }}=-30^{\circ}$ and $\phi_{\text {out }}=150^{\circ}$ via $\phi_{\text {out }}=60^{\circ}$ as forward scattering and scattering between the azimuth angles $\phi_{\text {out }}=150^{\circ}$ and $\phi_{\text {out }}=-30^{\circ}$ via $\phi_{\text {out }}=-120^{\circ}$ as backward scattering. In the angular distributions along the surface directions, negative $\theta=\theta_{\text {out }}$ denote backward scattering and positive forward scattering.

Figure 3.27 shows that the angular distributions in incidence direction assume a broad, cosine shaped form that peaks very close to the surface normal. For scattering along [12̄1] (orange),

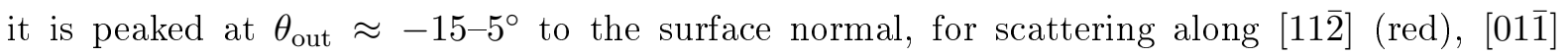

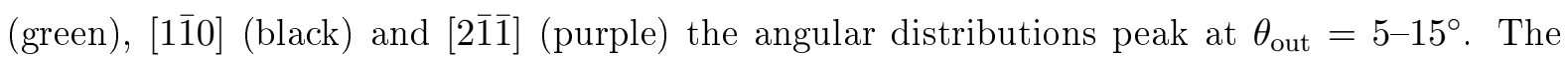
angular distribution for scattering along $[10 \overline{1}]$ is the broadest of the angular distributions and maintains a strong signal in $\theta_{\text {out }} \approx 5--30^{\circ}$ which is consistent with the intensity displayed in the total angular distribution (Fig. 3.16). Although peaked at slightly different polar angles,

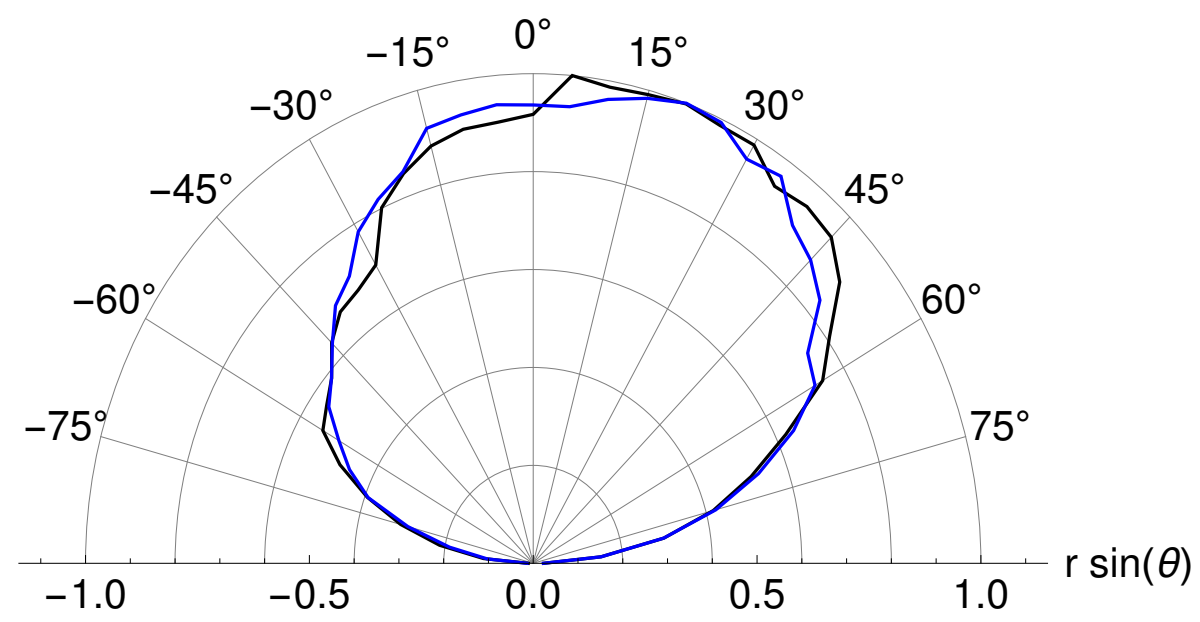

Figure 3.26.: Angular distribution along [101] -direction for the electronically adiabatic (blue) nonadi-

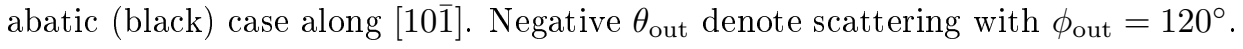




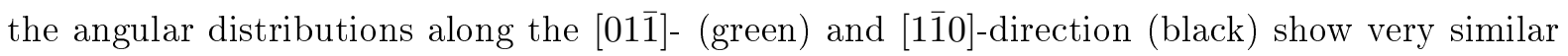

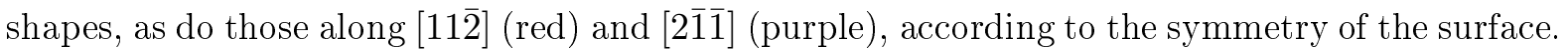
This symmetry is most likely also due to the low percentage of penetrating trajectories in the

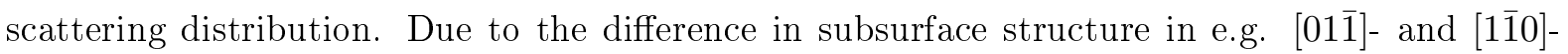
direction caused by the ABC-stacking, the penetrating trajectories have been observed to lead to differences in $\phi_{\text {out }}=60^{\circ} \pm \Delta \phi_{\text {out }}$ with the same $\Delta \phi_{\text {out }}$ for adiabatic simulations with higher incidence energies, both conditions which exhibit a higher fraction of penetratively scattering trajectories [3]. As to be expected, the strong intensity observed for forward scattering in [101] direction diminishes the further $\phi_{\text {out }}$ deviates from $60^{\circ}$, i.e., the more it tends towards backward scattering. The scattering along the [1리]-direction (orange) is almost centered around the surface normal; it marks the border between scattering into the forward and backward direction.

I studied the differential energy loss distribution dependent on the polar angle (Fig. 3.28) and azimuth angle (Fig. 3.29 in steps of $\theta_{\text {out }}=15^{\circ}$. Due to the noise is in the differential ELDs, especially for backward scattering, only very general trends can be extracted. It appears that, the closer to the incidence direction in forward scattering, the differential ELDs peak at the same energy losses, no matter $\theta_{\text {out }}$. For athwart and backward scattering, for $\theta_{\text {out }} \geq 45^{\circ}$, the peak and rising edge of the distribution move to slightly higher energy losses with growing polar scattering angle and the peak becomes less pronounced than for forward scattering. For backward scattering with growing $\theta$, the peak diminishes in favor of the shoulder, indicating a decrease of the single-bounce contributions in favor of the more-bounce contributions to the differential energy loss distribution. Furthermore, for forward scattering, the mean energy loss drops slightly towards higher polar scattering angles. This trend becomes less with sideways scattering until the mean energy loss remains almost constant. For backwards scattering, due to low signal to noise level, no clear trend can be extracted (see Tab. 3.12). That the trend is only seen for forward scattering is perhaps not surprising, as most of the single-bounce events happen in forward direction and single bounce events that do not dive deep into the electron density can be expected to retain most information on the incidence condition. In this case, they retain a little more of their normal energy than of their parallel energy.

\section{Summary}

In summary, the inclusion of electron hole pair excitation leads to very different energy loss, sticking and scattering behavior than the electronically adiabatic case, indeed both have almost nothing in common. They differ exceedingly in energy loss behavior, the energy loss mechanisms (as can be seen by the different ratios of bounces to one another), the sticking behavior and the mechanisms by which sticking occasioned: adiabatic and nonadiabatic scattering behavior are fundamentally different. 


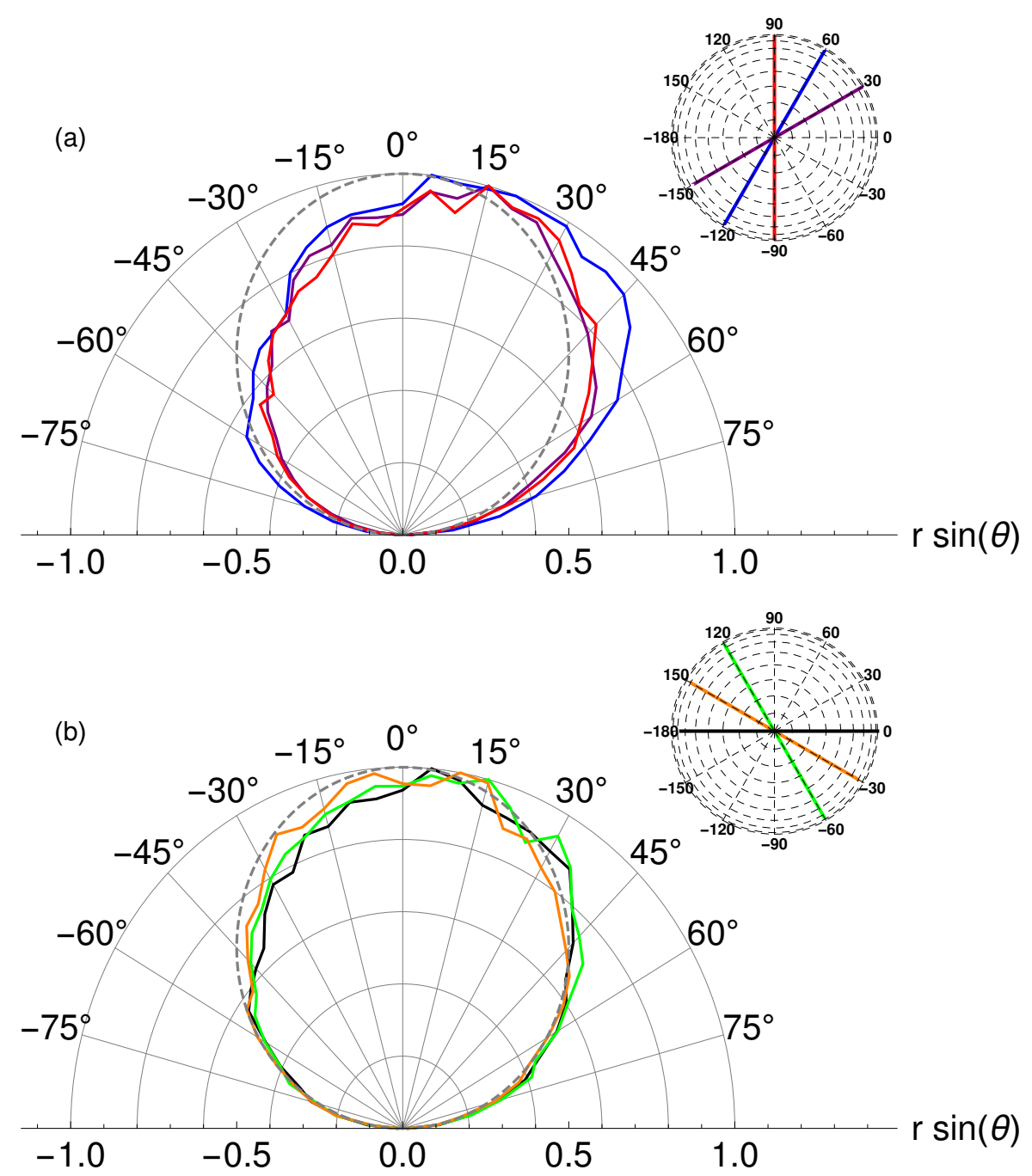

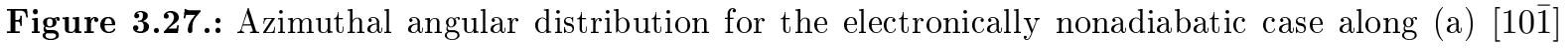

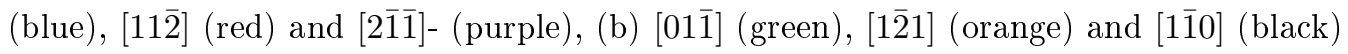
surface directions. Dashed gray corresponds to a cosine-distribution peaked at $0^{\circ}$. All curves were normalized to their respective maxima in intensity. The positive values of $\theta_{\text {out }}$ correspond to forward scattering, the negative ones backward scattering. The inset shows chosen scattering directions marked on the total angular distribution. A cosine distribution, centered at $\theta_{\text {out }}=0^{\circ}$ is given as a reference in dashed gray.

In the nonadiabatic case, the differential energy loss distribution due to forward scattering is peaked at low energy losses, a peak that corresponds to the peak of the differential energy loss distribution of the single-bounce events. Double-bounce events and multibounce events make up the (slight) shoulder and tail of the distribution and grow in importance the closer $\phi_{\text {out }}$ gets to backscattering. The influence of $\theta_{\text {out }}$ is small: for backscattering for larger $\theta_{\text {out }}$, the peak of the differential energy loss distribution shifts to slightly higher energy losses. The mean energy 

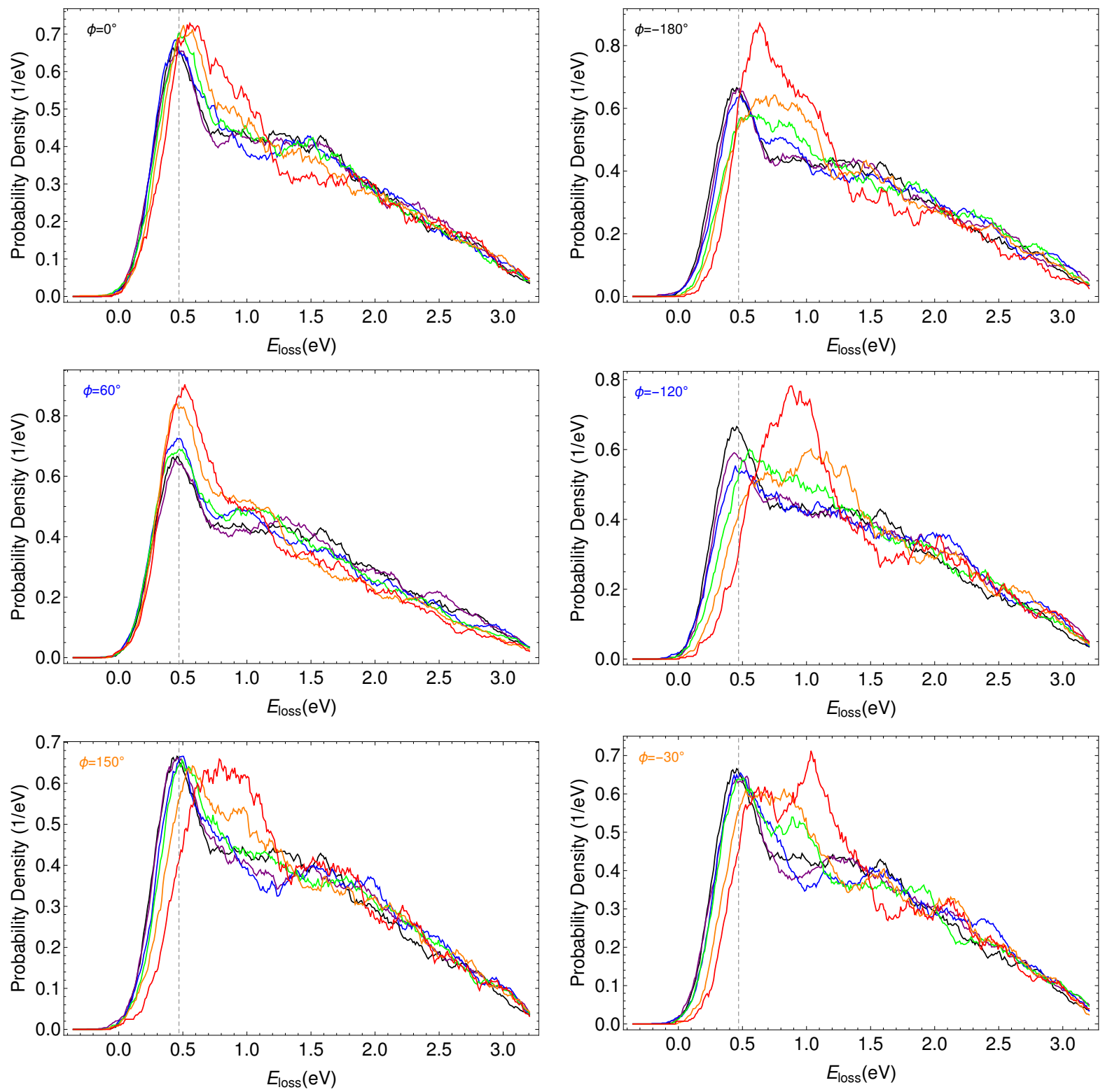

Figure 3.28.: Smoothed differential energy loss distribution for different exit polar angles (black: $0^{\circ}$, purple: $15^{\circ}$, blue: $30^{\circ}$, green: $45^{\circ}$, orange: $60^{\circ}$, red: $75^{\circ}$ ) for six exemplary exit azimuthal angles: $\phi_{\text {out }}=0^{\circ}$ and $\phi_{\text {out }}=-180^{\circ}, \phi_{\text {out }}=60^{\circ}$ and $\phi_{\text {out }}=-120^{\circ}$ and $\phi_{\text {out }}=150^{\circ}$ and $\phi_{\text {out }}=-30^{\circ}$. The gray dashed line marks the position of the peak in the total angular distribution.

loss drops with rising $\theta_{\text {out }}$ for forward scattering.

By analysis of the trajectories, I could find single-bounce as well as double-bounce collisions where the first impact was close to the a-top site. I was also able to show that different singlebounce events occurring further away from the top site yield slightly differently shaped differential energy loss distribution than those occurring very close to the a-top sites (see Tab. 3.12). 
Table 3.12.: Mean energy loss (in \% of incidence energy) for nonadiabatic simulations dependent on $\theta_{\text {out }}$ for different surface directions. Positive $\theta_{\text {out }}$ correspond to forward scattering and negative $\theta_{\text {out }}$ to backward scattering.

\begin{tabular}{lllll}
\hline \hline$\theta_{\text {out }}\left({ }^{\circ}\right)$ & {$[10 \overline{1}]$} & {$[11 \overline{2}]$} & {$[01 \overline{1}]$} & {$[1 \overline{2} 1]$} \\
\hline-60 & 39 & 42 & 40 & 41 \\
-45 & 40 & 41 & 41 & 40 \\
-30 & 42 & 41 & 41 & 40 \\
-15 & 40 & 41 & 41 & 40 \\
0 & 39 & 39 & 39 & 39 \\
15 & 39 & 39 & 39 & 40 \\
30 & 37 & 38 & 39 & 40 \\
45 & 37 & 37 & 39 & 40 \\
60 & 35 & 37 & 39 & 40 \\
\hline \hline
\end{tabular}

Although the differential energy loss distributions of the different scattering events overlap very much, the shape of the complete differential energy loss distribution changes with different scattering directions. A lowering of temperature may make it possible to distinguish between the different scattering events and shall be discussed in section 3.3 .2 For this, especially forward scattering appears to be promising as the peak due to the single-bounce events appears to be narrower here and might therefore be more clearly separated from the differential energy loss distributions of the other events. Due to this reason, I consider the following exit angles as especially promising: $\theta_{\text {out }}=35^{\circ}, \phi_{\text {out }}=10^{\circ}$ (strongest single bounce peak), $\theta_{\text {out }}=15^{\circ}$, $\phi_{\text {out }}=-35^{\circ}$ (largest separation between double and single bounce), and possibly scattering
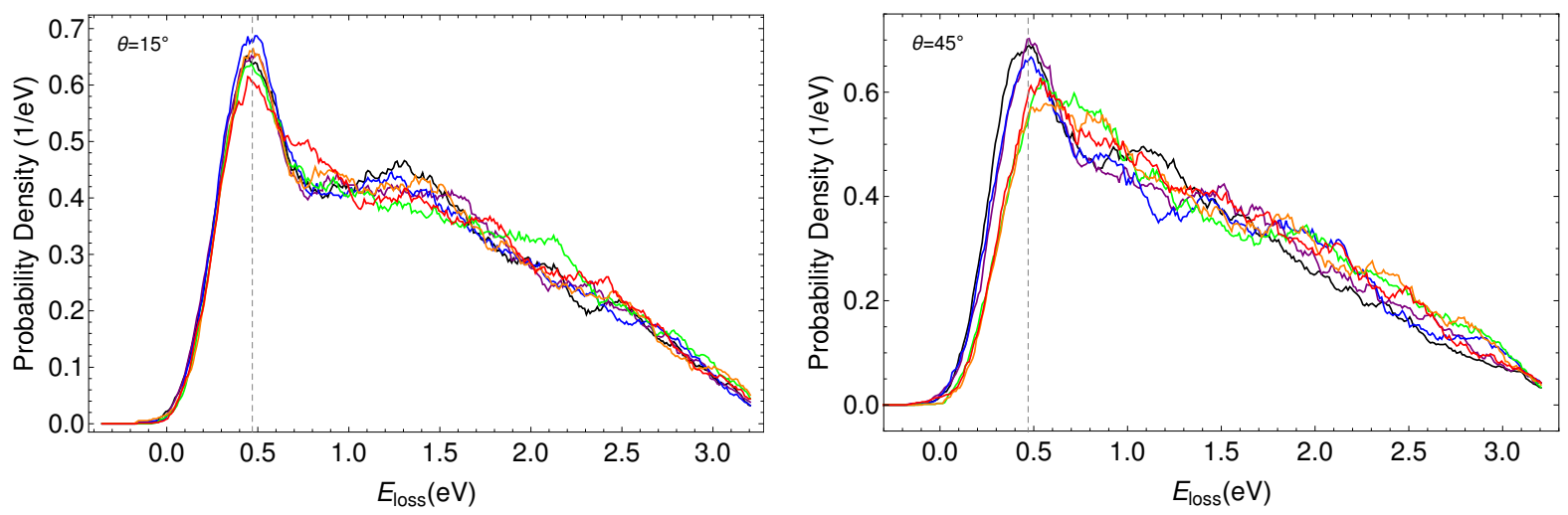

Figure 3.29.: Smoothed differential energy loss distribution for different azimuthal angles $\phi_{\text {out }}$ (black: $60^{\circ}$, purple: $0^{\circ}$, blue: $120^{\circ}$, green: $-60^{\circ}$, orange: $-180^{\circ}$, red: $-120^{\circ}$ ) for different two exemplary polar angles: $\theta_{\text {out }}=15^{\circ}$ and $\phi_{\text {out }}=45^{\circ}$. The gray dashed line marks the position of the peak in the total angular distribution. 
along the surface normal, $\theta_{\text {out }}=25^{\circ}, \phi_{\text {out }}=35^{\circ}$ and $\theta_{\text {out }}=50^{\circ}, \phi_{\text {out }}=65^{\circ}$.

The maximum in scattering intensity is along the incidence direction in forward direction, but is shifted closer to normal than the incidence beam.

It will be further interesting to investigate what becomes of the backwards scattering peaks if the incidence energy is changed: with a lowering of the incidence energy, it could be expected that more $\mathrm{H}$ atoms are scattered backwards (or take to sticking); a flatter incidence angle should also lead to more backwards scattering, whereas one closer to the surface normal will perhaps move the maximum in scattering intensity even further towards the surface normal.

\subsubsection{The Influence of Temperature on the Energy Loss Distribution}

In the nonadiabatic case, the fact that the peak of the energy loss distribution is almost entirely formed by the single-bounce events and the shoulder stems almost solely from the double-bounce events makes it appear likely that a lowering in temperature could result in a clear separation of these two events. This could then be verified with experimental studies and used to predict interesting exit conditions in experiment. I therefore analyze in this section the influence of temperature on the energy loss distribution. I have sampled the behavior of scattering and adsorption with temperature for $0,40,120,300,500$ and $700 \mathrm{~K}$. The scattering probabilities, ratio of bounce events and energy losses are presented in Tab.3.13 3.15. While the probabilities differ significantly for the electronically adiabatic and nonadiabatic calculations (as already described in Section 3.3.1), the change of probabilities for both cases due to the change in temperature is almost identical: Both for the electronically adiabatic and nonadiabatic case, the probability of scattering (Tab. 3.13 increases slightly with rising temperature while the probability of adsorption at the surface decreases. Absorption and transmission probability stay constant for the electronically adiabatic case. If excitation of ehp is included, the adsorption probability to the surface decreases with rising temperature, more significantly than for the adiabatic case and in favor of a larger subsurface adsorption. The findings for the electronically adiabatic case are in correspondence with the observations of Strömquist et al. [23] for $\mathrm{H}$ scattering from $\mathrm{Cu}(111)$ that absorption probabilities at high incidence energies and sticking probabilities do not show much variation with temperature.

The number of multibounce same as the probability of penetration decreases slightly with rising temperature (Tab. 3.14). In correlation with the slight decrease of multibounce events, the mean energy loss decreases with rising temperature, however, the peak of the energy loss distributions shift with increasing temperature to higher energy loss values, indicating an increase in high-energy single-bounce events (Tab3.15).

The total energy loss distribution for the different temperatures in the electronically nonadiabatic case (Fig. 3.30 (a)) illustrates that for temperatures below $300 \mathrm{~K}$, several peaks become discernible. While they are only barely visible for $120 \mathrm{~K}$ (green), they are almost separated at 
Table 3.13.: Outcomes (in \%) resulting from $\mathrm{H}$ atom collision with a $\mathrm{Au}(111)$ surface for nonadiabatic and adiabatic (in parenthesis) simulations for various temperature values. The incidence conditions are $E_{\text {inc }}=3.33 \mathrm{eV}, \theta_{\text {inc }}=45^{\circ}$ along the $[10 \overline{1}]$ surface direction, with a relaxed surface structure and $6 \times 6 \times 6$ slab, number of simulated trajectories: $10^{6}$.

\begin{tabular}{lllll}
\hline \hline Temperature & Scattering & $\begin{array}{l}\text { Surface } \\
\text { Adsorption }\end{array}$ & $\begin{array}{l}\text { Subsurface } \\
\text { Absorption }\end{array}$ & Transmission \\
\hline $0 \mathrm{~K}$ & $54(80)$ & $26(5)$ & $20(6)$ & $1(9)$ \\
$40 \mathrm{~K}$ & $54(80)$ & $26(5)$ & $20(6)$ & $1(8)$ \\
$120 \mathrm{~K}$ & $54(81)$ & $25(5)$ & $20(6)$ & $1(8)$ \\
$300 \mathrm{~K}$ & $55(82)$ & $23(4)$ & $21(6)$ & $1(8)$ \\
$500 \mathrm{~K}$ & $56(82)$ & $21(3)$ & $23(6)$ & $1(8)$ \\
$700 \mathrm{~K}$ & $56(83)$ & $19(3)$ & $24(6)$ & $1(9)$ \\
\hline \hline
\end{tabular}

$40 \mathrm{~K}$ (blue) and completely separated at $0 \mathrm{~K}$ (purple). The total energy loss distribution for the electronically adiabatic case (dashed lines, Figure 3.30(b)-(d)) broadens with rising temperature, but no clear separation between peaks is observable even at $0 \mathrm{~K}$. Figure $3.30(\mathrm{~b})-(\mathrm{d})$ clarifies that the peaks appearing in the electronically nonadiabatic case are due to energy losses occasioned by different bounce events: the first peak is solely caused by single-bounce events (blue). But even at $0 \mathrm{~K}$ (b), there is a small single-bounce contribution to the second peak of the total ELD that otherwise mostly consists of double-bounce events. The second peak, at $0 \mathrm{~K}$, is completely separated from the rest of the distribution which contains contributions due to

Table 3.14.: Outcomes (\%) of scattering for scattering events resulting from $\mathrm{H}$ atom collision with a $\mathrm{Au}(111)$ surface for nonadiabatic and adiabatic (in parenthesis) simulations for various temperature values. The "Surface"-column refers to trajectories wherein $\mathrm{H}$ atoms scattered from $1^{\text {st }}$ layer of the surface. The Roman numerals refer to the lowest subsurface to which penetration occurred. The incidence conditions are $E_{\text {inc }}=3.33 \mathrm{eV}, \theta_{\text {inc }}=45^{\circ}$ along the [101] surface direction and $6 \times 6 \times 6$ cell, number of simulated trajectories: $10^{6}$.

\begin{tabular}{llllllllll}
\hline \hline & \multicolumn{4}{l}{ bounce events } & \multicolumn{5}{c}{ penetrating bounces } \\
Temperature & single & double & multi & surface & I & II & III & $>$ III \\
\hline $0 \mathrm{~K}$ & $23(16)$ & $32(24)$ & $45(60)$ & $81(62)$ & $18(25)$ & $1(8)$ & $0(3)$ & $0(2)$ \\
$40 \mathrm{~K}$ & $23(16)$ & $32(24)$ & $45(60)$ & $81(62)$ & $19(25)$ & $1(8)$ & $0(3)$ & $0(2)$ \\
$120 \mathrm{~K}$ & $23(16)$ & $33(24)$ & $44(60)$ & $81(63)$ & $18(24)$ & $1(8)$ & $0(3)$ & $0(2)$ \\
$300 \mathrm{~K}$ & $23(17)$ & $34(25)$ & $43(59)$ & $82(64)$ & $17(23)$ & $1(8)$ & $0(3)$ & $0(2)$ \\
$500 \mathrm{~K}$ & $24(17)$ & $34(25)$ & $42(58)$ & $83(65)$ & $16(21)$ & $1(8)$ & $0(3)$ & $0(2)$ \\
$700 \mathrm{~K}$ & $25(18)$ & $34(25)$ & $41(57)$ & $84(65)$ & $16(21)$ & $1(8)$ & $0(3)$ & $0(3)$ \\
\hline \hline
\end{tabular}


Table 3.15.: Energy loss in \% of incidence energy for various outcomes resulting from $\mathrm{H}$ atom collision with a $\mathrm{Au}(111)$ surface for nonadiabatic and adiabatic simulations for various temperature values. The mean and maximum energy loss are shown for the total ELD and for scattering

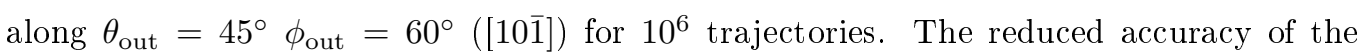
differential energy loss distributions reflects the lower signal to noise ratio of the differential ELD.

\begin{tabular}{lllll}
\hline \hline & Total & & \multicolumn{2}{l}{$\theta_{\text {out }}=45^{\circ} \phi_{\text {out }}=60^{\circ}$} \\
Temperature & Mean & Peak & Mean & Peak \\
\hline $0 \mathrm{~K}$ & $40.4(13.9)$ & $12.8(1.35)$ & $37(8.2)$ & $33(0.75)$ \\
$40 \mathrm{~K}$ & $40.4(13.8)$ & $13.4(1.35)$ & $37(9.9)$ & $13(1.1)$ \\
$120 \mathrm{~K}$ & $40.0(13.5)$ & $13.1(1.65)$ & $37(10)$ & $13(1.4)$ \\
$300 \mathrm{~K}$ & $39.22(13.1)$ & $14.0(1.65)$ & $37(10)$ & $14(0.75)$ \\
$500 \mathrm{~K}$ & $38.4(12.8)$ & $17.9(1.65)$ & $36(11)$ & $15(1.4)$ \\
$700 \mathrm{~K}$ & $37.7(12.6)$ & $16.7(2.25)$ & $35(11)$ & $14(1.4)$ \\
\hline \hline
\end{tabular}

double- and multibounce events and does not separate into individual peaks anymore. The clear separation into three contributions has ceased at $40 \mathrm{~K}$ (Fig. 3.30 (c)) and the low energy region of the total ELD of the double-bounces begins to overlap with the high energy loss region of the single bounce events, a process that increases with rising temperature.

With rising temperature, the total ELDs thus become more and more structureless (Fig. 3.30(a), (e) and (f); at $300 \mathrm{~K}$ (Fig. 3.30(a), yellow); the last remnant of the peak (mostly) resulting from the double-bounce events has turned into a shoulder and at $500 \mathrm{~K}$ (Fig. 3.30(e), orange) and $700 \mathrm{~K}$ (Fig. 3.30 (f), red) any distinct features are washed out: the total energy loss distribution of the single-bounce events now even distinctly overlaps with that of the multibounce events, and the peak of the total energy loss distribution has shifted to higher energy losses as already noted in Tab. 3.15. It is also to be noted that with increasing temperature, a higher number of $\mathrm{H}$ atoms scatter from the surface that have gained energy in the collision. Such events are probably to be imagined in such a manner that an $\mathrm{H}$ atom, upon collision with an Au atom, matches the atom's movement and by that is propelled further along on its path, gaining energy from the rising Au atom.

The distribution of scattering intensity also changes with rising temperature (Fig. 3.30(b)(f), insets): at $0 \mathrm{~K}$ (Fig. $3.30(\mathrm{~b})$ ), specular scattering is the most intense process, and almost all scattering happens along the forward direction, with little side-scattering. This pattern dissolves with rising temperature: the peak in scattering intensity moves more and more towards smaller polar angles and more side (and back-) scattering becomes apparent (Fig.3.30(c)-(f)). 

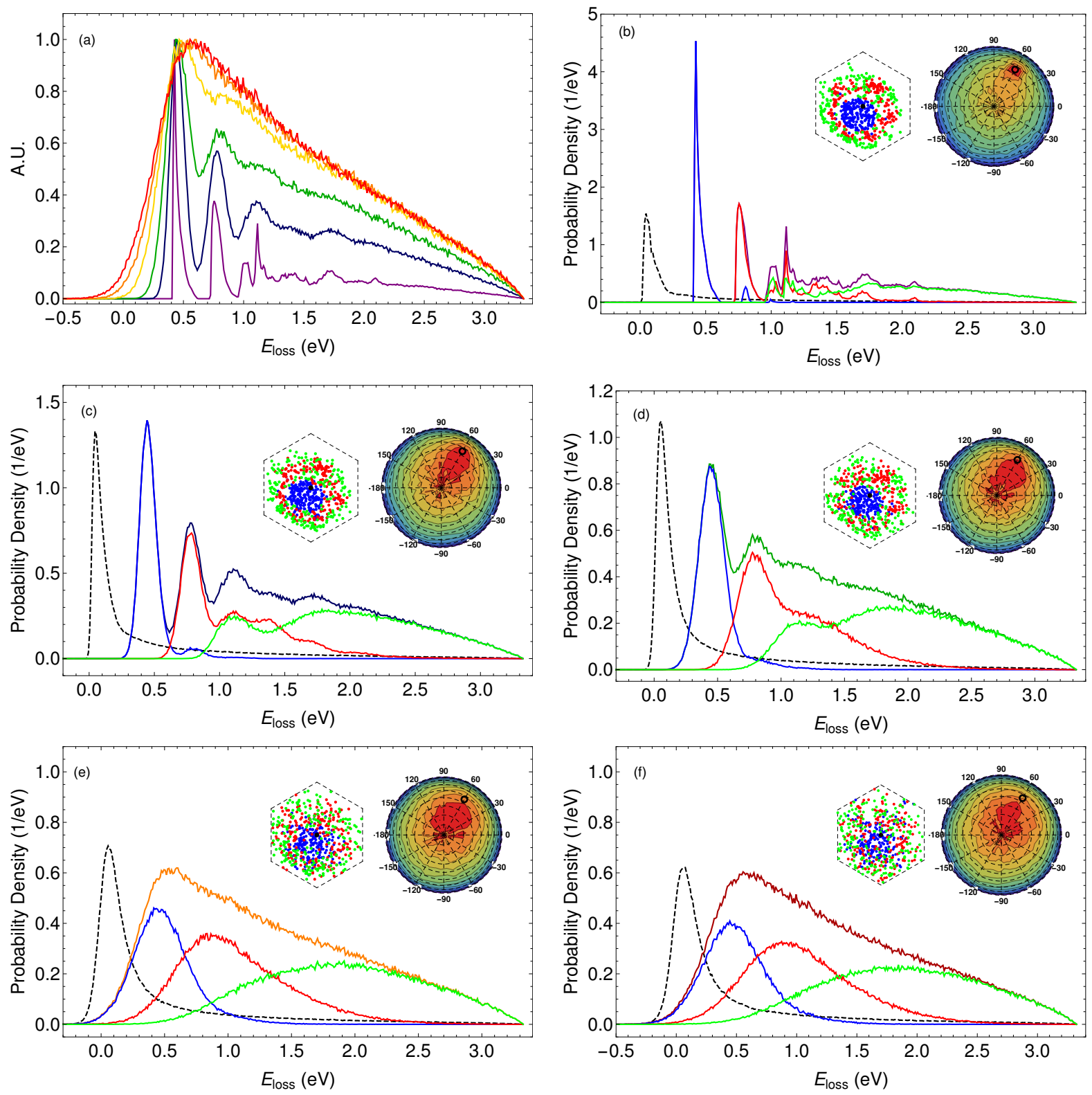

Figure 3.30.: (a) Nonadiabatic total energy loss distribution obtained from nonadiabatic simulations: resolved into contributions (a) total for $0 \mathrm{~K}$ (purple), $40 \mathrm{~K}$ (blue), $120 \mathrm{~K}$ (green), $300 \mathrm{~K}$ (yellow), $500 \mathrm{~K}$ (orange) and $700 \mathrm{~K}$ (red), normalized to maximum; and due to single(blue), double- (red) and multibounce (green) events at (b) $0 \mathrm{~K}$, (c) $40 \mathrm{~K}$, (d) $120 \mathrm{~K}$, (e) $500 \mathrm{~K}$ and (f) $700 \mathrm{~K}$. The dashed curve corresponds to the electronically adiabatic case (intensity decreased by 0.25 ) The insets show the sites of the bounce events reduced into the primitive cell for 200 trajectories each, a black dot marking the cells middle, and the total angular distribution for all scattering events at the respective temperatures. The polar scattering angle is denoted by the distance from the center of the plots starting with $\theta_{\text {out }}=0^{\circ}$ in the middle to $\theta_{\text {out }}=90^{\circ}$ at the outermost rim. $\phi_{\text {out }}$ is indicated explicitly. Red denotes $92 \%$ in scattering intensity and every contour denotes a drop by $1 / 12$ in intensity so that purple marks the regions of lowest intensity. The black circle indicates the specular scattering angle. 
At $700 \mathrm{~K}$, while the maximum in intensity is along forward scattering direction, the scattering intensity drops almost equally into all azimuthal directions with growing polar angle (Fig. 3.30(f)). The broadening of the angular distribution with growing temperature is related to the increasing motion of the surface atoms. At $0 \mathrm{~K}$ the Au surface is least corrugated which leads to specular scattering, with rising temperature, more of the Au atoms come out of the surface; the higher an Au atom is raised above the surface level during the collision with an $\mathrm{H}$ atom, the less likely the $\mathrm{H}$ atom is to leave the surface at high polar scattering angles.

The destructurization of the ELD due to the surface motion becomes very clear when the impact sites of single-, double- or multibounces are regarded: at low temperatures, a very clear structure is evident where collisions close to the center of the atom lead to single bounce events (Fig. 3.30(b), inset), the impact sites leading to double-bounce events form a corona around those leading to single-bounce collisions with bulges pointing in the direction of the bridgesites and therefore toward the neighboring atoms. These bulges are caused by double-bounces having a lower energy loss then $E_{\text {loss }}<0.94 \mathrm{eV}$, corresponding to the second peak of the total ELD, and are due to double-bounce events that have their second bounce with the nearest neighbors. At $0 \mathrm{~K}$ and $40 \mathrm{~K}$, this amounts to $100 \%$ of all double-bounce events of the second peak in the total ELD, genuinely identifying a mechanism by which this peak is caused. At higher temperatures, as the peak melts together with the other peaks of the total ELD, clear assignment disappears. The multibounce events are most removed from the a-top-site. The clear structuring of impact sites and a corresponding bounce event washes out with rising temperature (first insets Fig.3.30(c)-(f)), giving an indication of the motion of the Au atoms which, with the EMT-JAWK, can experience mean displacements of $0.22 \AA$ at $500 \mathrm{~K}$, corresponding to $5.2 \%$ of the lattice constant.

This trend in behavior becomes also clear when the angular distribution for forward scattering is considered (see Fig. 3.31): the angular distribution for $0 \mathrm{~K}$ (purple) is very narrow and peaks indeed very decisively at specular angle $\left(\theta_{\text {out }}=45^{\circ}\right)$. For $40 \mathrm{~K}$ (blue), the angular distribution becomes a little wider and peaks at $\theta_{\text {out }}=40^{\circ}$, for $120 \mathrm{~K}$ (green), it has assumed a very full shape and peaks at $25^{\circ}$, the angular distribution of $300 \mathrm{~K}$ peaks surprisingly much closer to the surface normal $\theta_{\text {out }}=5^{\circ},\left(\theta_{\text {out }}=20^{\circ}\right.$, yellow $)$ than that for $500 \mathrm{~K}\left(\theta_{\text {out }}=30^{\circ}\right.$, orange $)$, but this could be owing to the very flat peak the distribution shows at $300 \mathrm{~K}$, reaching from $\sim 5^{\circ}$ to $30^{\circ}$. The angular distribution for $700 \mathrm{~K}$ (red) peaks at $\theta_{\text {out }}=20^{\circ}$ and is very broad. All distributions show much more forward than backward scattering.

Fig. 3.32 shows a resolution of the angular distribution along the [101]-direction into the different bounce events. The sharp peak of the distribution is due to double-bounce events (red) which scatter almost exclusively at $\theta_{\text {out }}=45^{\circ}$ corresponding to specular angle. The multibounce (green) distribution shows a much wider distribution of scattering angles that peaks closer to the surface normal and the single-bounce distribution (blue) shows a preference for forward 


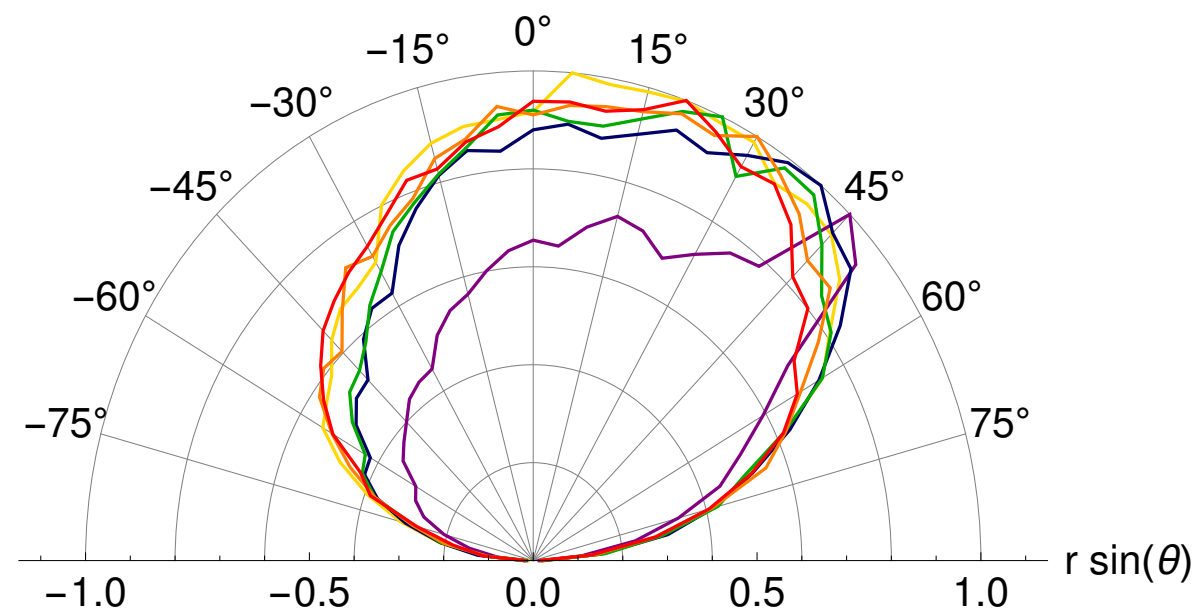

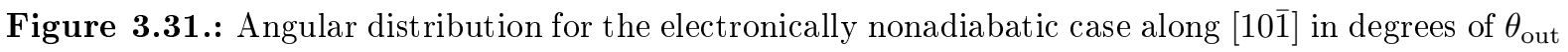
giving the surface direction. The positive angles correspond to forward scattering $\left(\phi_{\text {out }}=\right.$ $\left.60^{\circ}\right)$ and the negative ones to backward scattering $\left(\phi_{\text {out }}=-120^{\circ}\right)$. The dependence of the angular distribution on temperature is shown: purple: $0 \mathrm{~K}$, navy: $40 \mathrm{~K}$, dark green: $120 \mathrm{~K}$, yellow: $300 \mathrm{~K}$, orange: $500 \mathrm{~K}$ and red: $700 \mathrm{~K}$. Black: cosine distribution.

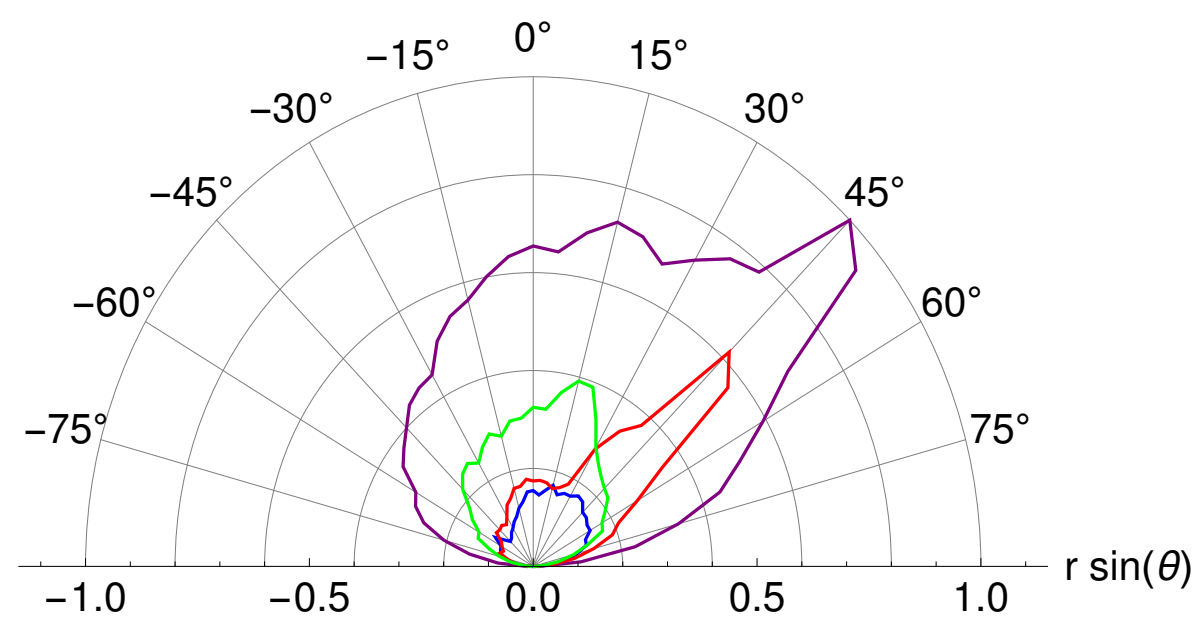

Figure 3.32.: Azimuthal angular distribution for the electronically nonadiabatic case at $0 \mathrm{~K}$ along $[10 \overline{1}]$ in degrees of $\theta_{\text {out }}, 0^{\circ}$ being the surface normal. The positive angles correspond to forward scattering $\left(\phi_{\text {out }}=60^{\circ}\right)$ and the negative ones to backward scattering $\left(\phi_{\text {out }}=-120^{\circ}\right)$. The contribution of different bounce events is shown: total (purple), single- (blue), double(red) and multibounce (green). The distributions are normalized to the maximum of the total angular distribution along [10̄1]. 

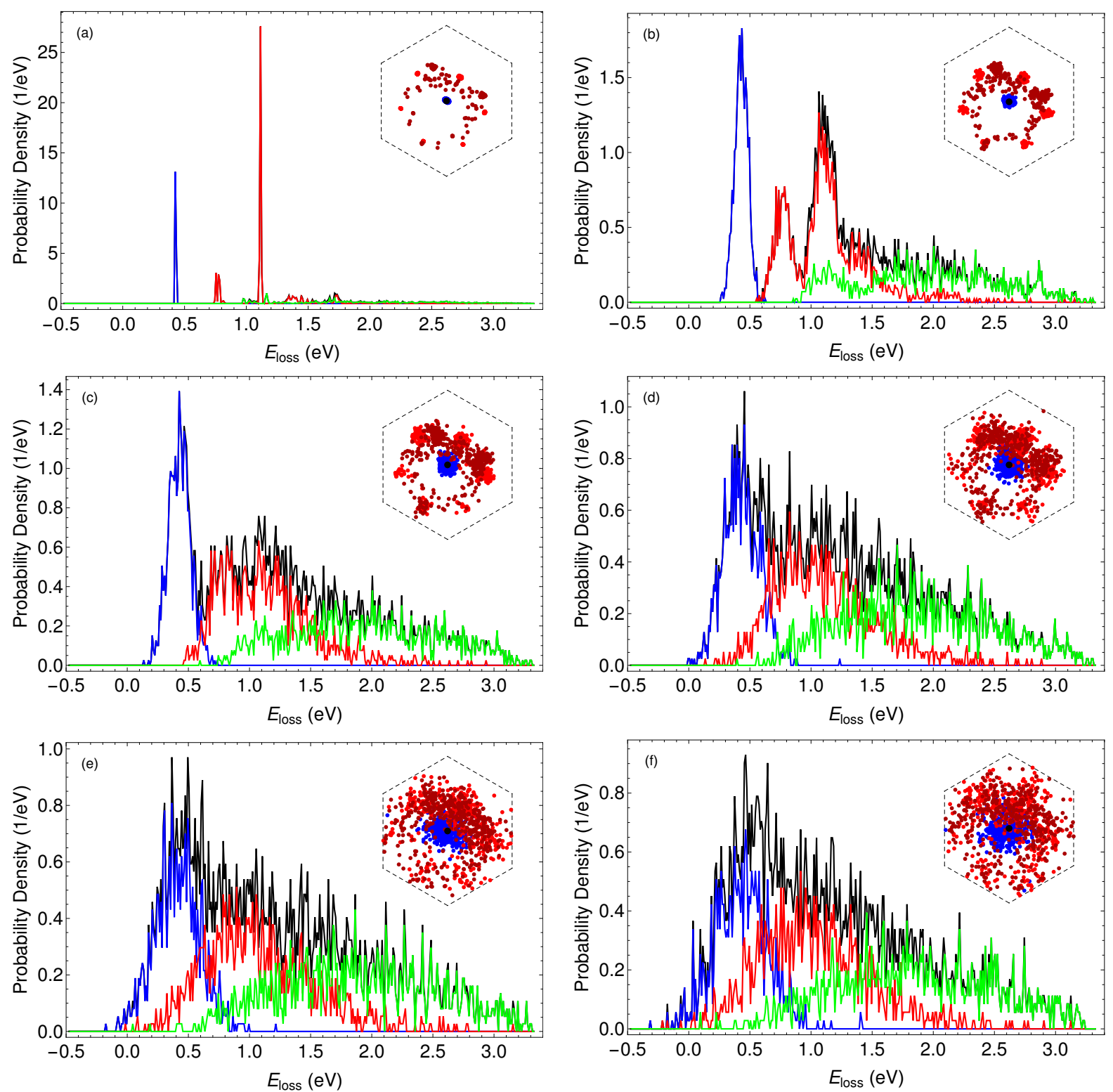

Figure 3.33.: Differential energy loss distribution for scattering along specular angles for (a) $0 \mathrm{~K}$, (b) $40 \mathrm{~K}$, (c) $120 \mathrm{~K}$, (d) $300 \mathrm{~K}$, (e) $500 \mathrm{~K}$, (f) $700 \mathrm{~K}$, due to total (black), single bounce (blue), double-bounce (red) and multibounce (green) scattering events. The inset shows the first impact site reduced into the primitive cell of the $\mathrm{H}$ atoms experiencing single- and doublebounce events and making up the first (blue), second peak (light red, all double-bounces $E_{\text {loss }}<0.94 \mathrm{eV}$ ) and rest due to double-bounce events (dark red).

direction, but no specific polar angle.

The question of whether the differential energy loss distributions due to different bounce events can be separated from one another by lowering the temperature is answered most satisfactorily: Fig. 3.33 shows a comparison of scattering along the specular angles for the different temperatures, resolved into different bounce events. At $0 \mathrm{~K}$ (Fig. $3.33(\mathrm{a})$ ), the first peak of the 
differential energy loss distribution is very narrow and sharply defined at $E_{\text {loss }} \approx 0.4 \mathrm{eV}$ energy loss, entirely made up from all the single-bounce events that contribute to this condition. These single-bounce events were the result of hitting a gold atom almost precisely at the a-top-site (observe blue points close to the center of primitive cell in Fig.3.33(a), inset). The second peak (at $E_{\text {loss }} \approx 0.75 \mathrm{eV}$ ) is made up entirely of double-bounce events that are not contaminated with any single-bounce events; as can be seen from the inset, these double-bounce events have their first impact at the side of the $\mathrm{Au}$ atom and exactly in the direction of the neighboring $\mathrm{Au}$ atoms (light red, all double-bounces up to $E_{\text {loss }}<0.94 \mathrm{eV}$ ) and indeed, all of these atoms collide with the next-neighbor atom. All other double-bounce events have a more dispersed first impact site (dark red). The third peak $\left(E_{\text {loss }} \approx 1.1 \mathrm{eV}\right)$ consists mostly of these double-bounce events, but is intermixed with some multibounce events. With increasing temperature, all three peaks begin to grow together: they do not appear to shift in position, but rather broaden out (Fig.3.33(b)-Fig. 3.33(f)).

The distinction between the second and third peak disappears for $T<120 \mathrm{~K}$ after which double-bounce events with $E_{\text {loss }}<0.94$ cease to form a clear orientational pattern towards the neighboring Au atoms, although it still remains guessable at $300 \mathrm{~K}$ (see also Fig. 3.21 (b), black arrows). At $40 \mathrm{~K}$ (Fig. $3.33(\mathrm{~b})$ ) the separation between the first and the second peak is still very clear. Since the first peak can solely be attributed to the differential energy loss distribution of single-bounce events, a separation between single- and double-bounce events is possible at $40 \mathrm{~K}$. A separation between double-bounce and multibounce events is not to be obtained; while the second peak mostly consists of the differential energy loss distribution due to double-bounce events, at its high energy loss side, it mixes with the low energy side of the multibounce differential energy loss distribution. Still, the clear separation into peaks may also be visible experimentally.

At $120 \mathrm{~K}$ (Fig. 3.33(c)), the second and third peak of the differential energy loss distribution merge into one. The first peak is now, at its high energy loss side, a mixture of contributions of the single- and double-bounce differential energy loss distribution: a clear assignment of peaks to either collision event becomes impossible at this temperature under these scattering angles. The differential energy loss distribution of the double-bounce events is now completely overlaid by that of the multibounce events. A further increase in temperature sees an overlap between the differential energy loss distributions of the single- and multibounce events; at $500 \mathrm{~K}$ (Fig. 3.33(e)), the differential energy loss distribution of the single-bounce events becomes completely overlaid by that of the double- and multibounce events which is even more pronounced at $700 \mathrm{~K}$ (Fig. 3.33 (f)).

For $T<120 \mathrm{~K}$ specular scattering angle is not the only one under which a separation of the differential energy loss distribution of the single-bounce and double-bounce events in energy loss can be achieved; indeed such a separation is to be seen at most scattering angles. To 
provide a few scattering angles that might, conceivably, be studied experimentally, Fig.3.34 shows a selection of such candidates. Fig. 3.34(a) shows the differential energy loss distribution for what was identified in Section 3.3.1 as the condition under which the largest separation between double- and single-bounce events could be achieved. Here, clearly, a good separation of the first peak from the rest of the distribution, made up only from energy losses due to single-bounce events, can be achieved. The separation is still not perfect, since the singlebounce part shows a small contribution beyond the first peak which intermixes with that of the double-bounce events. The same is true for normal scattering (Fig. 3.34(c)), identified in Section 3.3 .1 to be the area where the strongest contribution due to penetrating collisions was made, as well as for what was identified as the scattering conditions with the most intense double-bounce contribution (Fig. 3.34(d)). In the latter case, the lacking separation between double- and single-bounce differential energy loss distributions becomes even more pronounced. For the scattering angles identified in Section 3.3.1 to have the most intense single-bounce peak (Fig. 3.34(b)), the first peak also shows contribution from the double-bounce differential energy loss distribution, due to a small contribution from the double-bounce differential energy loss distribution at $E_{\text {loss }} \approx 0.48 \mathrm{eV}$. For the three cases $(\mathrm{a}),(\mathrm{c})$ and $(\mathrm{d})$, the separation of the first peak from other contributions but single-bounce is so great that it can be regarded as complete; while it would be desirable to find a condition under which full separation of the single-, doubleand multibounce differential energy loss distribution could be achieved, this appears to be to little avail since the separation between double- and multibounce differential energy loss distribution cannot be affected: Fig. 3.34(e) shows the scattering angle where the largest separation between double- (red) and multibounce (green) differential energy loss distributions at a reasonable signalto noise ratio happened.

The conditions when the first peak of the differential energy loss distribution made up from contributions of only single-bounce events can be mostly achieved for all $\theta_{\text {out }}$ for $\phi_{\text {out }}$ between $-175^{\circ}$ and $150^{\circ}$; the most complete separation between the single- and double-bounce differential energy loss distribution is to be had at $\theta_{\text {out }} \approx 10^{\circ}$ for $\phi_{\text {out }}$ between -165 and $\phi_{\text {out }}=135^{\circ}$, and $\phi_{\text {out }}$ from -30 to $40^{\circ}$. To separate the double- and multibounce differential energy loss distribution entirely is impossible, but Fig. 3.35 shows a few fine examples of exit conditions where the largest separation between first (only single-bounce), second (only double-bounce) and rest (mixture of double and multibounce contributions) peaks can be achieved. If these conditions could be proved experimentally and the differential energy loss distribution showed similar behavior, a fine assignment of peaks could be accomplished.

For $120 \mathrm{~K}$, neither a separation of single- and double-bounce contributions nor one of a first peak made up only of single-bounce contributions from the rest of the distribution is possible. The overlap between the single- and double-bounce differential energy loss distribution that can be seen in Fig. 3.33(d) is on the order of the smallest overlap that can be found; while it is clear 

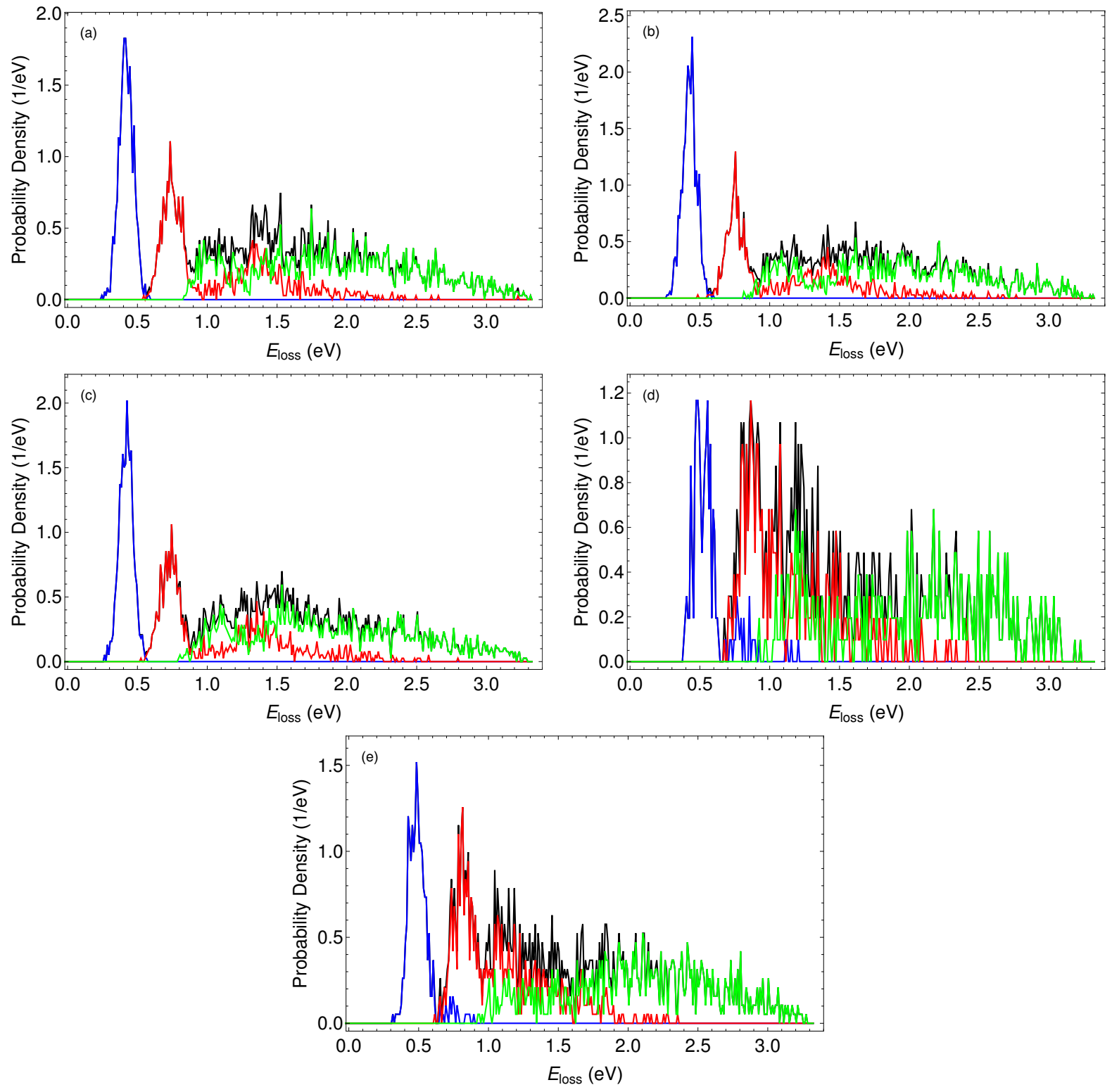

Figure 3.34.: Differential energy loss distribution for $\mathrm{T}=40 \mathrm{~K}$ for different scattering angles: (a) $\theta_{\text {out }}=15^{\circ}, \phi_{\text {out }}=-35^{\circ}$ (b) $\theta_{\text {out }}=35^{\circ}, \phi_{\text {out }}=10^{\circ}$, (c) $\theta_{\text {out }}=0^{\circ}$, (d) $\theta_{\text {out }}=75^{\circ}$, $\phi_{\text {out }}=-25^{\circ}$, (e) $\theta_{\text {out }}=60^{\circ}, \phi_{\text {out }}=-35^{\circ}$, resolved in total (black), single bounce (blue), double-bounce (red) and multibounce (green) scattering events.

that most of the first peak is due to single-bounce scattering events, especially the high-energy loss side of the peak is also influenced by double-bounce collisions and in this case even by a small contribution due to the multibounce differential energy loss distribution.

For a number of scattering angles, the first peak of the differential energy loss distribution can be attributed to single-bounce collisions alone while the second peak in a few cases can be attributed to double-bounce collisions with nearest neighbor atoms in a very few selected cases 

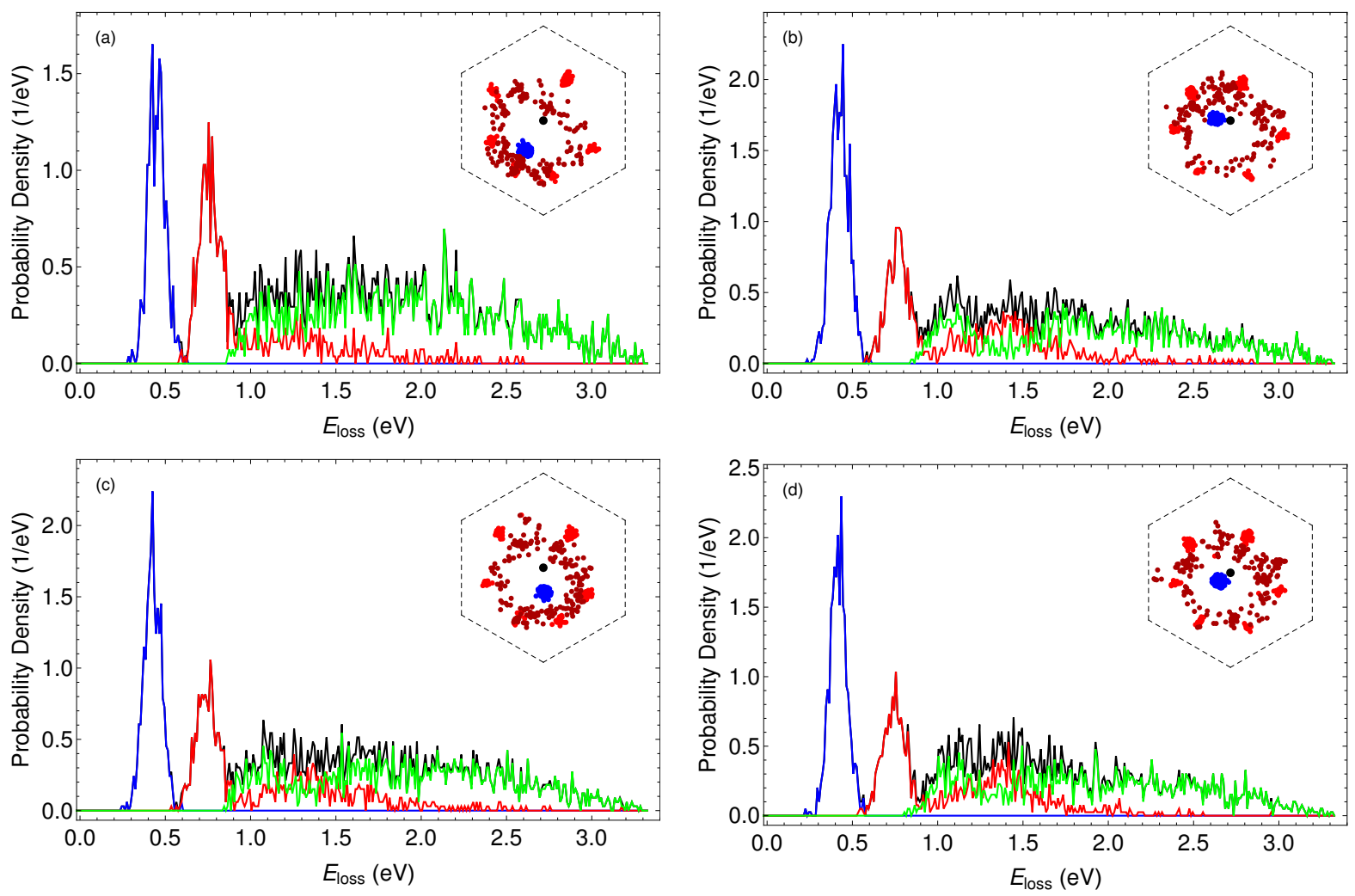

Figure 3.35.: Differential energy loss distribution of exit conditions at $T=40 \mathrm{~K}$ that are promising for experimentally probing: (a) $\theta_{\text {out }}=35^{\circ}, \phi_{\text {out }}=-120^{\circ}$, (b) $\theta_{\text {out }}=40^{\circ}, \phi_{\text {out }}=95^{\circ}$, (c) $\theta_{\text {out }}=25^{\circ}, \phi_{\text {out }}=-30^{\circ}$, (d) $\theta_{\text {out }}=20^{\circ}, \phi_{\text {out }}=90^{\circ}$. The first peak is entirely due to single-bounce events (blue), the second one entirely to double-bounce events with the nearest neighbors (red) and the rest due to multi- (green) and other double-bounce events. In the inset, the sites of the 200 first impact are shown. Here, the double-bounce events not contributing to the second peak are marked in dark red.

at $40 \mathrm{~K}$. Seeing that the separation is not possible at all exiting angles, I believe that raising the temperature much above $40 \mathrm{~K}$ will make an assignment of one peak being due to one kind of collision event already impossible. At $120 \mathrm{~K}$, the overlap between the single- and double-bounce differential energy loss distribution is large already for most scattering angles and even at those where it is comparatively low, an overlap of $\Delta E_{\text {loss }} \approx 0.3 \mathrm{eV}$ already exists.

In any case, with the lowering of the temperature, the peaks due to the different bounce events begin to separate. If the theory presented here predicts the correct thermal behavior, already a small lowering of temperature should show the emergence of peak structures which, at much lower temperatures, can be assigned to certain bounce events. 


\subsubsection{The Influence of Incidence Energy}

I performed MD simulations with the EMT-JAWK PES for a wide range of incidence energies from $0.1 \mathrm{eV}$ to $10.0 \mathrm{eV}$ (Tab.3.16-3.18). $0.99-3.33 \mathrm{eV}$ being incidence energies that have been accessed experimentally.

In Tab. 3.16 and Fig. 3.36 (a), the dependence of the scattering probability with incidence energy is shown. The electronically adiabatic and nonadiabatic case show again many differences; the scattering (black) probability is higher for the adiabatic case than for the nonadiabatic case while the sticking probability (orange) to the surface is lower. For both adiabatic and nonadiabatic case, the scattering probability (black) rises with increasing incidence energy, very strongly at low incidence energies and more gradually at larger ones. The scattering probability levels off to a constant scattering probability of $80 \%$ above $3.0 \mathrm{eV}$ in the adiabatic case and to $\sim 60 \%$ above $5.0 \mathrm{eV}$ in the nonadiabatic case. The general similarity notwithstanding, in the electronically nonadiabatic case, there is barely any reflection at $E_{\text {inc }}=0.1 \mathrm{eV}$, while in the electronically adiabatic case, already $14 \%$ are reflected at this incidence energy.

In the same manner, the sticking probability to the surface (orange) sees a much sharper decrease with rising incidence energy in the adiabatic case than in the nonadiabatic case. The

Table 3.16.: Outcomes (in \%) resulting from $\mathrm{H}$ atom collision with a $\mathrm{Au}(111)$ surface for nonadiabatic and adiabatic (in parenthesis) simulations. The incidence conditions are $\theta_{\text {inc }}=45^{\circ}$ along the $[10 \overline{1}]$ surface direction, $T=300 \mathrm{~K}$ with $6 \times 6 \times 6$ slab, number of simulated trajectories: nonadiabatic: $10^{6}$, adiabatic: $10^{5}$.

\begin{tabular}{lllll}
\hline \hline & Scattering & $\begin{array}{l}\text { Surface } \\
\text { Adsorption }\end{array}$ & $\begin{array}{l}\text { Subsurface } \\
\text { Absorption }\end{array}$ & Transmission \\
\hline \hline 0.1 & $1(14)$ & $88(59)$ & $11(27)$ & $0(0)$ \\
0.5 & $9(49)$ & $77(31)$ & $14(20)$ & $0(0)$ \\
0.77 & $15(61)$ & $69(22)$ & $16(17)$ & $0(1)$ \\
0.99 & $20(66)$ & $63(18)$ & $17(16)$ & $0(1)$ \\
1.22 & $26(69)$ & $56(15)$ & $18(15)$ & $0(2)$ \\
1.92 & $39(76)$ & $41(9)$ & $20(12)$ & $0(4)$ \\
2.17 & $43(77)$ & $37(8)$ & $20(11)$ & $0(5)$ \\
2.62 & $49(79)$ & $30(6)$ & $21(9)$ & $0(6)$ \\
2.76 & $50(80)$ & $29(5)$ & $21(8)$ & $0(7)$ \\
3.33 & $55(81)$ & $23(4)$ & $21(7)$ & $1(8)$ \\
5.0 & $64(84)$ & $13(1)$ & $22(3)$ & $2(12)$ \\
7.0 & $67(83)$ & $8(0)$ & $20(1)$ & $4(15)$ \\
10.0 & $70(81)$ & $6(0)$ & $18(0)$ & $6(19)$ \\
\hline \hline
\end{tabular}


Table 3.17.: Outcomes (\%) of scattering for various scattering events resulting from $\mathrm{H}$ atom collision with a $\mathrm{Au}(111)$ surface for nonadiabatic and adiabatic (in parenthesis) simulations. The "Surface"-column refers to trajectories wherein the H atoms scattered from the first layer of the surface. The Roman numerals refer to the lowest subsurface to which penetration occurred. The incidence conditions are $\theta_{\text {inc }}=45^{\circ}$ along the $[10 \overline{1}]$ surface direction, $T=$ $300 \mathrm{~K}$ with $6 \times 6 \times 6$ cell, number of simulated trajectories: nonadiabatic: $10^{6}$, adiabatic: $10^{5}$.

\begin{tabular}{lllllllll}
\hline \hline & \multicolumn{6}{l}{ bounce events } & \multicolumn{6}{c}{ penetrating bounces } \\
$E_{\text {inc }}(\mathrm{eV})$ & single & double & multi & surface & I & II & III & $>$ III \\
\hline 0.1 & $96(40)$ & $4(26)$ & $0(34)$ & $100(99)$ & $0(1)$ & $0(0)$ & $0(0)$ & $0(0)$ \\
0.5 & $72(17)$ & $24(25)$ & $4(57)$ & $100(92)$ & $0(8)$ & $0(0)$ & $0(0)$ & $0(0)$ \\
0.77 & $53(16)$ & $38(26)$ & $9(58)$ & $99(86)$ & $1(13)$ & $0(1)$ & $0(0)$ & $0(0)$ \\
0.99 & $43(16)$ & $42(27)$ & $14(57)$ & $98(83)$ & $2(16)$ & $0(1)$ & $0(0)$ & $0(0)$ \\
1.22 & $37(16)$ & $44(27)$ & $19(57)$ & $96(80)$ & $4(18)$ & $0(2)$ & $0(0)$ & $0(0)$ \\
1.92 & $29(16)$ & $40(26)$ & $31(58)$ & $92(73)$ & $8(22)$ & $0(4)$ & $0(1)$ & $0(0)$ \\
2.17 & $27(16)$ & $39(26)$ & $34(58)$ & $90(71)$ & $10(23)$ & $0(5)$ & $0(1)$ & $0(0)$ \\
2.62 & $25(16)$ & $36(26)$ & $38(58)$ & $87(68)$ & $13(23)$ & $0(7)$ & $0(2)$ & $0(1)$ \\
2.76 & $25(16)$ & $36(25)$ & $39(58)$ & $86(67)$ & $14(23)$ & $1(7)$ & $0(2)$ & $0(1)$ \\
3.33 & $23(17)$ & $34(25)$ & $43(59)$ & $82(64)$ & $17(23)$ & $1(8)$ & $0(3)$ & $0(2)$ \\
5.00 & $22(17)$ & $29(22)$ & $49(61)$ & $74(58)$ & $23(22)$ & $3(10)$ & $0(5)$ & $0(5)$ \\
7.00 & $24(18)$ & $25(20)$ & $52(62)$ & $69(54)$ & $25(22)$ & $6(10)$ & $1(6)$ & $0(7)$ \\
10.0 & $28(19)$ & $21(18)$ & $51(63)$ & $66(50)$ & $26(23)$ & $7(11)$ & $2(7)$ & $0(8)$ \\
\hline \hline
\end{tabular}

greatest apparent difference between the two cases is the absorption probability (blue): it drops continuously in the adiabatic case, while it rises first in the nonadiabatic case, and only starts to drop very gradually above $E_{\text {inc }} \approx 5 \mathrm{eV}$. Inclusion of the transmission probability into the absorption probability (yellow) shows that this behavior is due to deeper bulk penetration at higher incidence energies, and that the absorption probability including transmission is independent of the incidence energy when $E_{\mathrm{inc}}>3.0 \mathrm{eV}$ and almost the same for the adiabatic and nonadiabatic case. The trends observed for the electronically adiabatic case at low incidence energies agree with the observations Strömquist at al. 23] made for the adiabatic interaction of $\mathrm{H}$ with $\mathrm{Cu}(111)$.

The contributions of the different collision events in the scattering process also differs in the adiabatic and nonadiabatic case (Fig. 3.36 (b)). Similarities are that at low incidence energies, single-bounces dominate over the other scattering events and at higher incidence energies multibounce events over the others. With rising incidence energy, single-bounce events become even less frequent, their proportion stays approximately constant when $E_{\text {inc }}>2.0 \mathrm{eV}$ in the nonadia- 
Table 3.18.: Energy loss in \% of incidence energy for various outcomes resulting from $\mathrm{H}$ atom collision with a $\mathrm{Au}(111)$ surface for nonadiabatic and adiabatic simulations for various incidence energies values. The incidence conditions are $\theta_{\mathrm{inc}}=45^{\circ}$ along the $[10 \overline{1}]$ surface direction, $T=300 \mathrm{~K}$ with $6 \times 6 \times 6$ cell, number of simulated trajectories: nonadiabatic: $10^{6}$, adiabatic: $10^{5}$. The mean and maximum energy loss are shown for total and differential ELD. The accuracy at specular scattering angles $\left(\theta_{\text {out }}=45^{\circ} \phi_{\text {out }}=60^{\circ}([10 \overline{1}])\right)$ is reduced due to the lower signal-to-noise ratio of the differential ELDs.

\begin{tabular}{lllll}
\hline \hline & Total & & \multicolumn{2}{l}{$\theta_{\text {out }}=45^{\circ} \phi_{\text {out }}=60^{\circ}$} \\
$E_{\text {inc }}(\mathrm{eV})$ & Mean & Peak & Mean & Peak \\
\hline 0.1 & $18.7(7.56)$ & $45.0(5.00)$ & $16(6.5)$ & $65(5.0)$ \\
0.5 & $42.1(18.2)$ & $39.0(3.00)$ & $40(14)$ & $35(1.0)$ \\
0.77 & $42.1(16.3)$ & $35.7(1.95)$ & $42(13)$ & $64(2.0)$ \\
0.99 & $42.3(15.2)$ & $27.8(2.53)$ & $40(12)$ & $28(2.5)$ \\
1.22 & $42.3(14.6)$ & $25.0(2.87)$ & $38(12)$ & $23(0.41)$ \\
1.92 & $41.5(13.9)$ & $20.6(1.82)$ & $38(11)$ & $21(1.8)$ \\
2.17 & $41.1(13.6)$ & $17.3(1.61)$ & $39(9.6)$ & $18(2.5)$ \\
2.62 & $40.4(13.5)$ & $15.8(2.10)$ & $37(11)$ & $14(2.1)$ \\
2.76 & $40.1(13.4)$ & $15.4(1.63)$ & $37(12)$ & $15(0.54)$ \\
3.33 & $39.2(13.1)$ & $14.0(1.65)$ & $37(11)$ & $14(0.75)$ \\
5.00 & $36.8(12.0)$ & $14.1(1.50)$ & $33(9.5)$ & $11(1.1)$ \\
7.00 & $38.0(10.0)$ & $23.6(1.50)$ & $35(8.2)$ & $11(1.64)$ \\
10.0 & $49.1(7.68)$ & $46.7(1.25)$ & $42(7.1)$ & $25(1.3)$ \\
\hline \hline
\end{tabular}

batic case and $E_{\mathrm{inc}}>0.5 \mathrm{eV}$ in the adiabatic case. After rising sharply at low incidence energies in the nonadiabatic case the probability of double-bounce events drops towards higher incidence energies in favor of multibounce events. These trends can be rationalized with the relation between incidence energy and surface geometry. At low incidence energies experiencing more than one collision will make it difficult to retain enough energy to escape the surface again (in the nonadiabatic case more so than in the electronically adiabatic case); single-bounce events are favored. The higher the incidence energy, the more likely for $\mathrm{H}$ atoms to experience higher number of collisions to escape the surface; the probability of double-bounce events first increases and then drops in favor of the multibounce events. That the probability for single-bounce collision stays almost constant is most likely due to that at higher incidence energies, some $\mathrm{H}$ atoms that before were pulled into poly-collision events retain so much initial energy that they are flung from the surface before they can lose enough energy to undergo a further collision.

The penetration probability rises with increasing incidence energy, because larger incidence energy allows an atom that penetrates the surface to lose more of its initial energies in interaction 

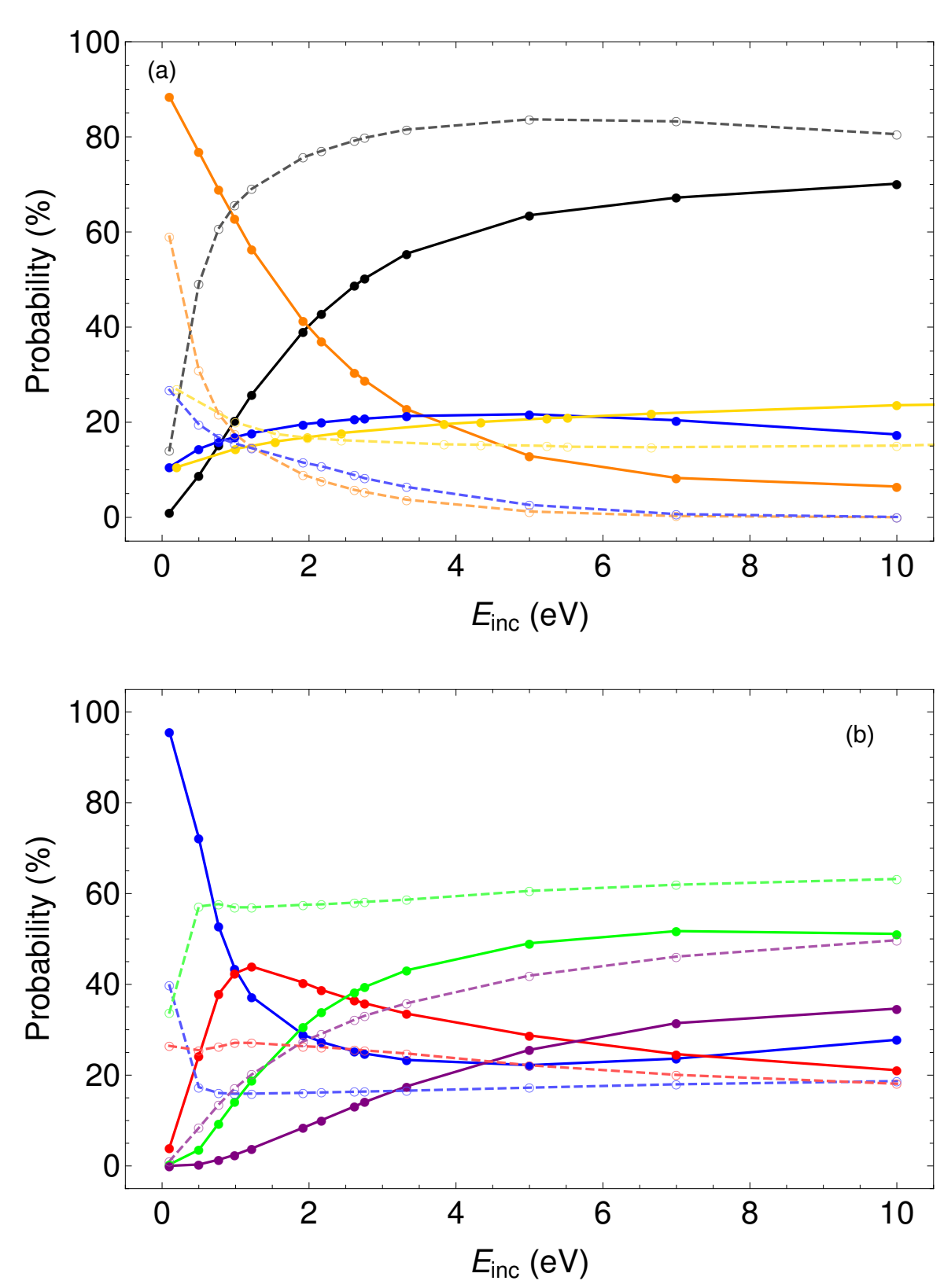

Figure 3.36.: Dependence of outcomes (in \%) of MD simulations of H scattering from $\mathrm{Au}(111)$ on the incidence energy. Panel (a) shows scattering (black), adsorption to the surface (orange), absorption into the surface (blue) and absorption including transmission (yellow). Panel (b) shows outcomes for events: single- (blue), double- (red), multi- (green) and penetrating bounces (purple) for the electronically nonadiabatic (solid lines and closed circles) and adiabiabatic (light dashed lines and open circles) cases.

with subsurface regions and still retain enough energy to scatter back. In the nonadiabatic case the rise is slower than in the adiabatic case; in the nonadiabatic case, any $\mathrm{H}$ atom venturing below the surface must expect to be so effectively slowed down by the friction force of the surrounding electron density that it needs much energy or very short subsurface penetration to 

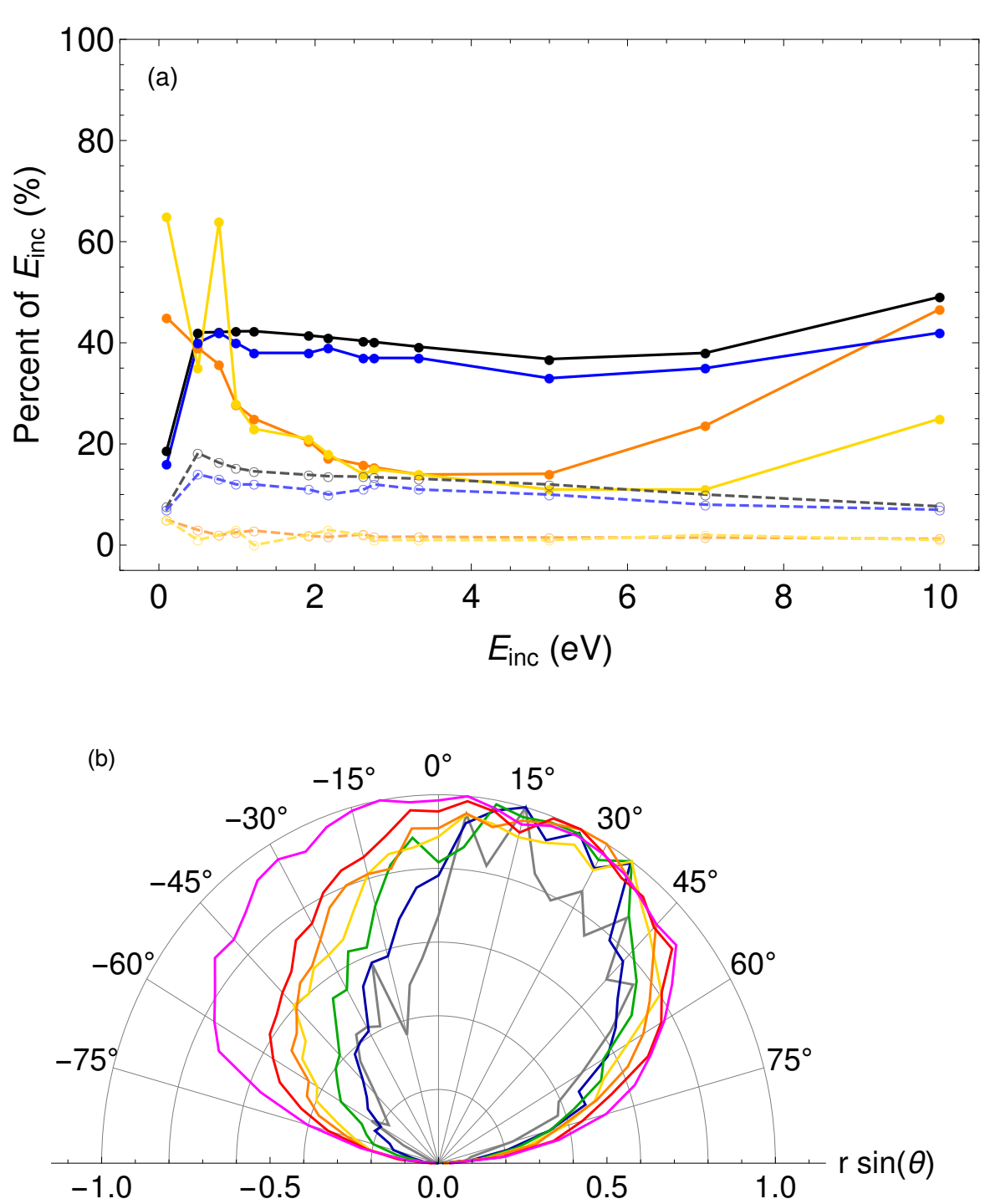

Figure 3.37.: (a) Dependence of the energy loss on the incidence energy (in percent of incidence energy): mean energy loss (black) for all trajectories, energy loss at peak (orange) for all trajectories, mean energy loss at specular scattering (blue), energy loss at peak of specular scattering (yellow). Electronically nonadiabatic with closed circles, adiabatic dashed and with open circles. (b) Angular distribution along the [101] -direction of $E_{\text {inc }}=0.1$ (grey), 0.99 (dark blue), 2.17 (dark green), 2.76 (yellow), 5.0 (orange), 7.0 (red), $10.0 \mathrm{eV}$ (magenta).

escape this influence. Similarly, the larger the incidence energy, the more likely it becomes to penetrate into the surface and scatter off successively deeper subsurface layers (Tab. 3.17).

As can be seen from Tab. 3.18 and from Fig.3.37(a), the mean energy loss for the total case (black) and for the specular scattering case (blue) rises at low incidence energies to $\bar{E}_{\text {loss }} \sim 15 \%$ in the adiabatic case and $\bar{E}_{\text {loss }} \sim 40 \%$ in the nonadiabatic case and then in the adiabatic case drops gradually for higher incidence energies. In the nonadiabatic case, the mean energy 


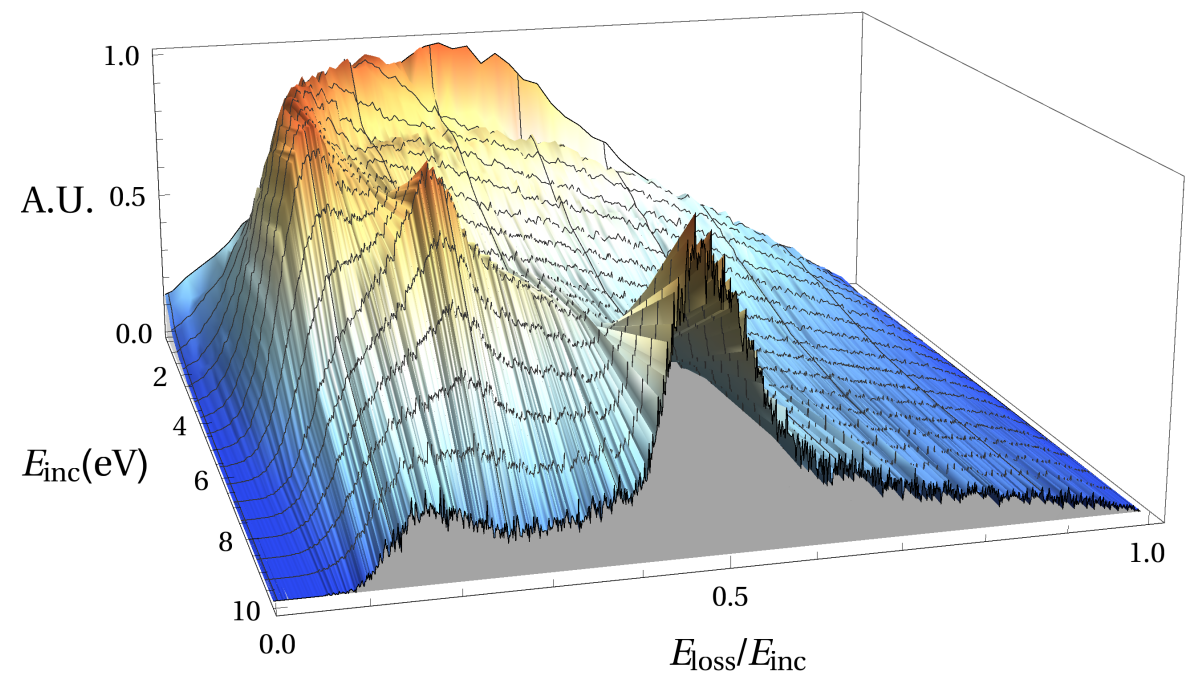

Figure 3.38.: Total energy loss distributions for different incidence energies in a 3-D plot. The total ELD of $0.1 \mathrm{eV}$ is not shown as the signal is too weak.

loss increases again for very high incidence energies. This means that at $E_{\text {inc }}=10 \mathrm{eV}$, the mean energy loss in the nonadiabatic case is six times larger than the adiabatic mean energy loss, compared to three times larger at lower incidence energies. That the mean energy loss at specular angle is lower than that of total ELD is occasioned by the fact that scattering into forward direction at large $\theta_{\text {out }}$ experiences lower energy losses than into backwards direction (see Section 3.3.1, e.g. Fig. 3.29 and below).

The relative energy loss at the position of the first peak is identical for the total energy loss distribution (orange, dashed) and that of specular (yellow, dashed) scattering for the adiabatic case. In the nondiabatic case, the two differ considerably. At low energy losses, this is due to the low signal-to-noise ratio (because most of the $\mathrm{H}$ atoms stick to the surface and cannot contribute to the energy loss distribution of the scattering trajectories) which makes it difficult to identify the peak position. At high energy losses, the divergence is due to the differential energy loss distribution of specular angles not containing all the events that lead to the formation of the total energy loss distributions and again a decrease of the signal-to-noise ratio as the maximum of the scattering distribution shifts from forward towards backwards scattering with $E_{\text {inc }}>5 \mathrm{eV}$ (see Fig. 3.37(b) and inset on Fig. 3.40(1) and (m)). As can be seen from Fig.3.38(c), the shape of the total ELD changes with increasing incidence energies; first, only a single peak is to be seen. With the rising percentage of double and multibounces, a shoulder begins to appear next to the peak at higher energy losses in the total ELD which, towards higher incidence energies, begins to turn into a peak itself and becomes much stronger than the peak at low energy losses.

Figures 3.39 and 3.40 show the total energy loss distribution for the different incidence energies resolved into the bounce events. They underscore what Fig. 3.37 and Fig. 3.38 already show, that 

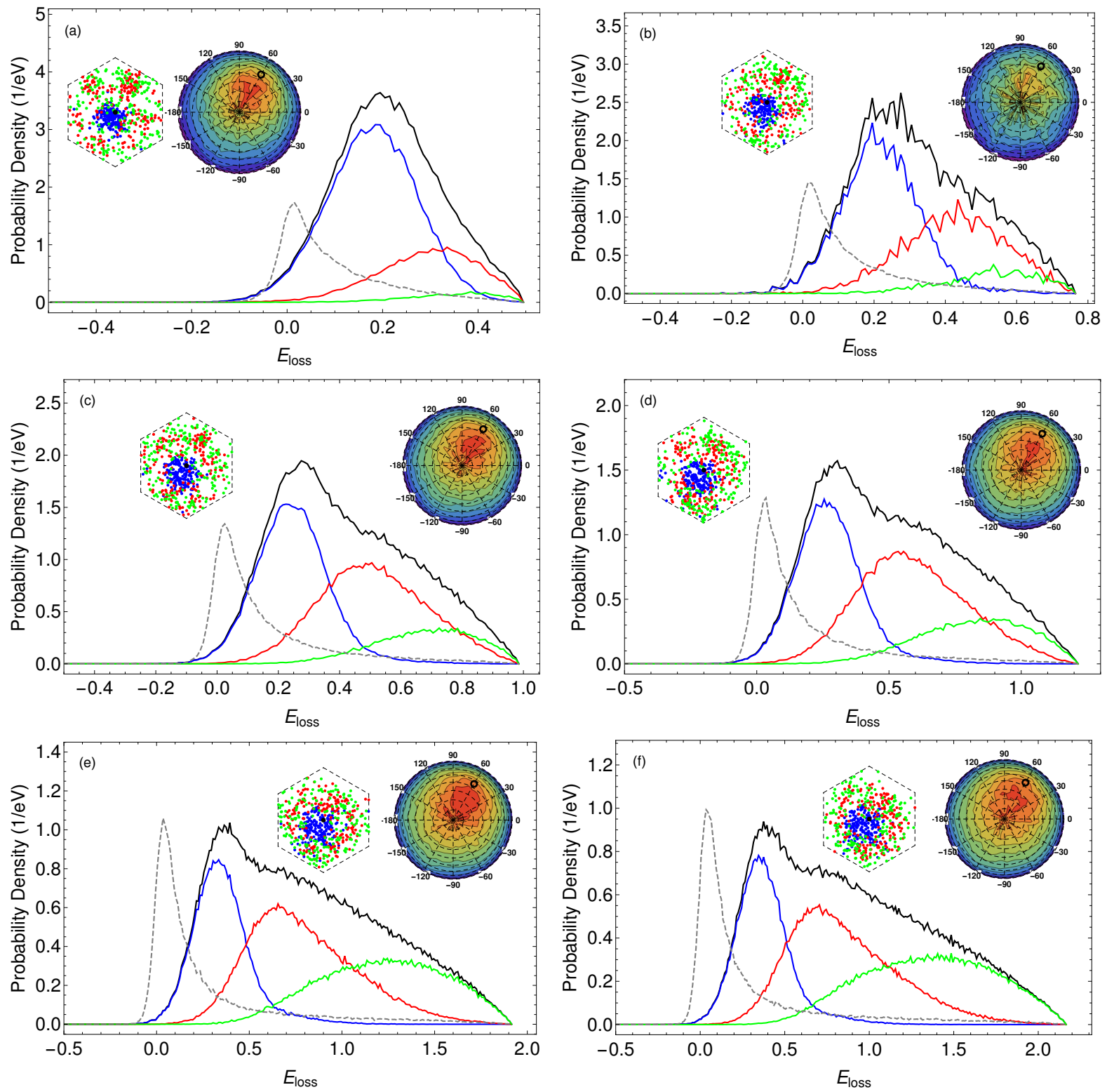

Figure 3.39.: Total energy loss distribution for the incidence energies of $0.5 \mathrm{eV}$ (a), $0.77 \mathrm{eV}$ (b), $0.99 \mathrm{eV}$ (c), $1.22 \mathrm{eV}$ (d), $1.92 \mathrm{eV}$ (e), $2.17 \mathrm{eV}$ (f). The gray dashed line shows the adiabatic total ELD, its probability density scaled by $0.25 \mathrm{eV}$. The insets show the primitive cell with the 200 first impact sites of single- (blue), double- (red) and multiple (green) bounces and the total angular distribution. The polar scattering angle is denoted by the distance from the center of the plots starting with $\theta_{\text {out }}=0^{\circ}$ in the middle to $\theta_{\text {out }}=90^{\circ}$ at the outermost rim. $\phi_{\text {out }}$ is indicated explicitly. Red denotes $>92 \%$ in scattering intensity and every contour denotes a drop by $1 / 12$ in intensity so that purple marks the regions of lowest intensity. The black circle indicates the specular exiting angle. For $0.1 \mathrm{eV}$ the ELD is not shown, due to the weak signal. 

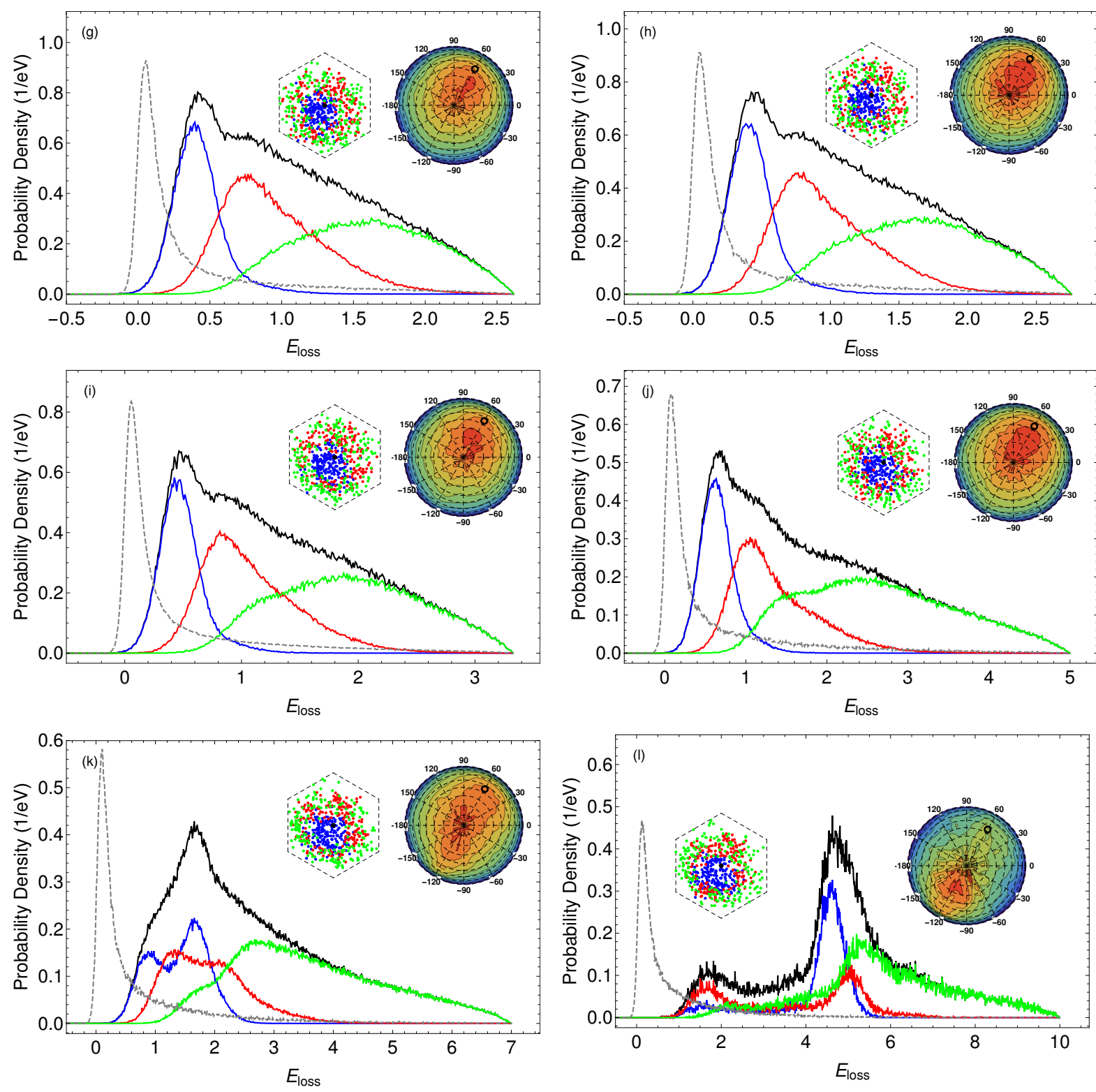

Figure 3.40.: Total energy loss distribution for incidence energies of $2.62 \mathrm{eV}(\mathrm{g}), 2.76 \mathrm{eV}(\mathrm{h}), 3.33 \mathrm{eV}(\mathrm{j})$, $5.0 \mathrm{eV}(\mathrm{k}), 7.0 \mathrm{eV}(\mathrm{l}), 10.0 \mathrm{eV}(\mathrm{m})$. The gray dashed line shows the adiabatic total ELD, its probability density scaled by $0.25 \mathrm{eV}$. The inset shows the primitive cell and the 200 first impact sites of single- (blue), double- (red) and multiple (green) bounce events, and the energy integrated total angular distribution.

the peak of the total energy loss distribution shifts to consecutively higher energies with rising incidence energies, that the nascent double- and multibounce total energy loss distributions push out a shoulder in the total ELD with rising incidence energy that becomes more and more dominant at high incidence energies, is joined by high-energy single-bounce contributions and at $10 \mathrm{eV}$ finally becomes the dominating peak. 
Table 3.19.: Exit angles under which high intensity can be expected for different incidence energies and the electronically nonadiabatic case. Negative $\theta_{\text {out }}$ denote scattering into the general backwards direction.

\begin{tabular}{lll}
\hline \hline$E_{\text {inc }}(\mathrm{eV})$ & $\theta_{\text {out }}\left({ }^{\circ}\right)$ & $\phi_{\text {out }}\left({ }^{\circ}\right)$ \\
\hline 0.1 & 0.0 & 0.0 \\
0.5 & $0-30$ & $50-80$ \\
0.77 & 25 & 75 \\
0.92 & $0-35$ & $30-90$ \\
1.22 & $0-30$ & $50-85$ \\
1.92 & $0-40$ & $0-120$ \\
2.17 & $0-35$ & $30-90$ \\
2.62 & $0-35$ & $30-105$ \\
2.76 & $0-40$ & $-10-130$ \\
5.0 & $-10-40$ & $0-150$ \\
7.0 & $-20-20$ & all \\
& $-40-40$ & $30-90$ and $-90--150$ \\
10.0 & $0-35$ & $-90--150$ \\
\hline \hline
\end{tabular}

At $E_{\text {inc }}=0.1 \mathrm{eV}$ (Fig. $3.37(\mathrm{~b})$ ), the scattering intensity in [101] direction is very narrow with almost no scattering in backwards direction. This is to be expected as, at very low incidence energies, a $\mathrm{H}$ atom that leaves the surface at a very low angle or in backwards direction will lose so much energy due to the interaction with the electron hole pairs that it will be unable to leave the surface. At $E_{\text {inc }}=0.5 \mathrm{eV}$ (Fig. 3.39 (a)), the maximum in scattering intensity is already roughly in the $[10 \overline{1}]$-direction and $\theta_{\text {out }} \approx 10^{\circ}$. From $E_{\text {inc }}=0.5 \mathrm{eV}$ upwards, the highest

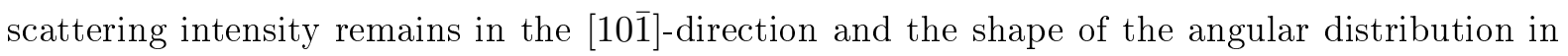
this direction (Fig. 3.37(b)) broadens. At the same time, sidewards and backwards scattering increases. At very high incidence energies $\left(>E_{\text {inc }}=5.0 \mathrm{eV}\right)$, the backwards scattering peak becomes more intense until at $E_{\text {inc }}=7.0 \mathrm{eV}$ (Fig.3.37(b), red and Fig.3.39(k)) scattering into forward and backward direction appears nearly equally intense. At $E_{\text {inc }}=10.0 \mathrm{eV}$ ((Fig. $3.39(1)$ ) and Fig. 3.37(b)), magenta), scattering into backwards direction is more intense than into forward direction.

The second single-bounce peak that appears at $E_{\text {inc }}>5.0 \mathrm{eV}$ is caused by $\mathrm{H}$ atoms hitting the surface closer to the hollow sites than those that form the first peak of the total ELD. The single-bounce trajectories with a higher energy loss $\left(E_{\text {loss }}>1.2 \mathrm{eV}\right.$ for $E_{\text {inc }}=7.0 \mathrm{eV}$ and $E_{\text {loss }}>4.0 \mathrm{eV}$ for $E_{\text {inc }}=10.0 \mathrm{eV}$ ) scatter without exception into backwards direction while those with lower energy loss scatter exclusively in forward direction. As to why those scattering into 
backwards direction lose so much more energy as those scattering into forward direction (and thereby increase the mean energy loss so much compared to the adiabatic case), I expect that the ones scattering into forward direction 'skim' over the Au-atom and are flung by their large incidence energy into forward direction, only gracing the atom. In contrast, the high incidence energy allows atoms that scatter closer to the hollow sites to dive far deeper into the electron density and thereby lose a great part of their incidence energy.

From the total angular distribution, the best signal for observing scattering with various incidence energies are shown in Tab.3.19. For the incidence energies $1.22 \mathrm{eV}-5$. eV, the maximum in intensity is around $\theta_{\text {out }}=25^{\circ}$ and $\phi_{\text {out }}=60^{\circ}$. The trend in higher incidence energies to scatter especially backwards makes them hard to observe since the detector would have to be placed together with the incidence beam. In these cases, the best observation position would be as far as possible towards backward or along the surface normal. Certainly, it would be most interesting to do experimental studies at very high incidence energy values so if the predictions made here for the growing of a new peak can be matched.

\subsubsection{The Dependence of Scattering on the Incidence Angles}

To see if the incidence angle has any influence on the scattering behavior and thus predict incidence (and corresponding exit) conditions that either show a separation of the differential energy loss distributions due to the different bounce events or other interesting features which could be investigated experimentally, I have performed MD simulations for the incidences angle

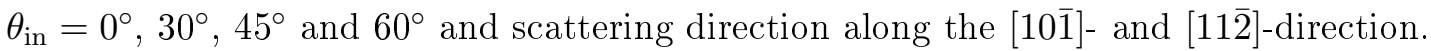

From Tab. 3.20 it can be seen that the scattering probability increases with the incidence angle,

Table 3.20.: Outcomes (in \%) resulting from $\mathrm{H}$ atom collision with a $\mathrm{Au}(111)$ surface for nonadiabatic and adiabatic (in parenthesis) simulations for various incidence angles. The incidence energy is $E_{\text {inc }}=3.33 \mathrm{eV}$, with a relaxed surface and $6 \times 6 \times 6$ slab at $300 \mathrm{~K}$, number of simulated trajectories: $10^{6}$.

\begin{tabular}{lllll}
\hline \hline & Scattering & $\begin{array}{l}\text { Surface } \\
\theta_{\text {in }}, \phi_{\text {in }}\left(^{\circ}\right)\end{array}$ & $\begin{array}{l}\text { Subsurface } \\
\text { Adsorption }\end{array}$ & Transmission \\
\hline 0,0 & $53(79)$ & $22(4)$ & $25(7)$ & $1(10)$ \\
30,60 & $52(79)$ & $23(4)$ & $25(7)$ & $1(10)$ \\
30,90 & $52(79)$ & $23(4)$ & $24(7)$ & $1(10)$ \\
45,60 & $55(82)$ & $23(4)$ & $21(6)$ & $1(8)$ \\
45,90 & $56(82)$ & $23(4)$ & $20(6)$ & $0(8)$ \\
60,60 & $64(87)$ & $21(3)$ & $15(5)$ & $0(6)$ \\
60,90 & $62(82)$ & $22(4)$ & $15(6)$ & $0(8)$ \\
\hline \hline
\end{tabular}


Table 3.21.: Outcomes (\%) of scattering for scattering events resulting from $\mathrm{H}$ atom collision with a $\mathrm{Au}(111)$ surface for nonadiabatic and adiabatic (in parenthesis) simulations for various incidence energies. The "Surface"-column refers to trajectories wherein the H atoms scattered from $1^{\text {st }}$ layer of the surface. The Roman numerals refer to the lowest subsurface to which penetration occurred. The incidence conditions are $E_{\text {inc }}=3.33 \mathrm{eV}, 300 \mathrm{~K}$ and $6 \times 6 \times 6$ slab, number of simulated trajectories: $10^{6}$.

\begin{tabular}{llllllllll}
\hline \hline & \multicolumn{4}{l}{ bounce events } & \multicolumn{5}{c}{ penetrating bounces } \\
$\theta_{\text {in }}, \phi_{\text {in }}\left(^{\circ}\right)$ & single & double & multi & surface & I & II & III & $>$ III \\
\hline 0,0 & $23(16)$ & $30(22)$ & $47(63)$ & $72(54)$ & $25(28)$ & $3(12)$ & $0(3)$ & $0(2)$ \\
30,60 & $23(16)$ & $31(22)$ & $46(62)$ & $78(59)$ & $20(25)$ & $1(10)$ & $0(4)$ & $0(2)$ \\
30,90 & $23(16)$ & $31(22)$ & $46(62)$ & $78(59)$ & $21(26)$ & $1(9)$ & $0(4)$ & $0(3)$ \\
45,60 & $23(17)$ & $34(25)$ & $43(59)$ & $82(64)$ & $17(23)$ & $1(8)$ & $0(3)$ & $0(2)$ \\
45,90 & $22(16)$ & $33(25)$ & $44(59)$ & $80(63)$ & $19(25)$ & $1(7)$ & $0(3)$ & $0(2)$ \\
60,60 & $28(22)$ & $36(29)$ & $36(49)$ & $89(75)$ & $11(17)$ & $0(5)$ & $0(2)$ & $0(1)$ \\
60,90 & $23(17)$ & $39(25)$ & $39(59)$ & $85(64)$ & $15(23)$ & $0(8)$ & $0(3)$ & $0(2)$ \\
\hline \hline
\end{tabular}

reaching over $60 \%$ at $\theta_{\text {in }}=60^{\circ}$ in the electronically nonadiabatic case and over $80 \%$ at $\theta_{\text {in }}=60^{\circ}$ in the adiabatic case. This tendency is more evident in nonadiabatic case. The azimuth incidence direction has no consistent influence on scattering probabilities. The adsorption probability to

Table 3.22.: Energy loss in \% of incidence energy for various outcomes resulting from $\mathrm{H}$ atom collision with a $\mathrm{Au}(111)$ surface for nonadiabatic and adiabatic simulations for various incidence angles. The incidence conditions are $E_{\text {inc }}=3.33 \mathrm{eV}, 300 \mathrm{~K}$ and $6 \times 6 \times 6$ slab, number of simulated trajectories: $10^{6}$. The mean and maximum energy loss are shown for the total ELD and for scattering at $\theta_{\text {out }}=45^{\circ}$ and the two incidence direction [101] $\left(\phi_{=} 60^{\circ}\right)$ and $[11 \overline{2}]\left(\phi_{=} 90^{\circ}\right)$. The reduced accuracy is due to the low signal to noise ratio in the differential ELDs.

\begin{tabular}{lllllll}
\hline \hline & \multicolumn{2}{l}{ Total } & & \multicolumn{2}{l}{$\theta_{\text {out }}=45^{\circ} \phi_{\text {out }}=60^{\circ}$} & \multicolumn{2}{l}{$\theta_{\text {out }}=45^{\circ} \phi_{\text {out }}=90^{\circ}$} \\
$\theta_{\text {in }}, \phi_{\text {in }}\left(^{\circ}\right)$ & Mean & Peak & Mean & Peak & Mean & Peak \\
\hline 0,0 & $39.1(14.5)$ & $14.0(2.25)$ & $39(15)$ & $14(2.3)$ & $40(16)$ & $11(2.3)$ \\
30,60 & $39.2(14.5)$ & $14.6(1.95)$ & $38(13)$ & $15(1.1)$ & $38(14)$ & $14(1.1)$ \\
30,90 & $39.3(14.5)$ & $14.0(1.95)$ & $38(14)$ & $11(1.7)$ & $38(13)$ & $17(1.4)$ \\
45,60 & $39.2(13.1)$ & $14.0(1.65)$ & $37(11)$ & $14(0.75)$ & $37(12)$ & $15(1.1)$ \\
45,90 & $39.5(13.0)$ & $14.3(1.95)$ & $38(12)$ & $16(1.4)$ & $37(11)$ & $14(1.4)$ \\
60,60 & $38.5(10.0)$ & $16.4(1.35)$ & $36(7.8)$ & $14(0.75)$ & $36(9.1)$ & $15(0.75)$ \\
60,90 & $39.6(10.8)$ & $26.9(1.65)$ & $37(9.7)$ & $13(1.1)$ & $37(8.9)$ & $15(1.1)$ \\
\hline \hline
\end{tabular}


the surface remains also roughly the same for all incidence directions. Seeing that the surface is only partly populated by direct adsorption and much by resurfacing processes (see Sec.3.3.1), this outcome is not surprising. The probability to absorb subsurface decreases with growing polar incidence angle; at high polar angle, the Au atoms overlap more to the incoming $\mathrm{H}$ atoms, and thus shield the hollow sites, making it difficult for incoming $\mathrm{H}$ atoms to penetrate the surface (see Tab. 3.21, penetration probability and scattering from subsurface layers); this is also reflected in the lowering of the probability to experience multibounce events at high polar angles.

The mean energy loss (Tab. 3.22) of the scattering trajectories remains constant for the total energy loss distribution of the nonadiabatic case. In forward scattering direction, the mean energy loss drops marginally with growing polar incidence angle. That this behavior is not reflected in the total ELD is most likely due to the circumstance that, in the nonadiabatic case, all events not scattering in forward direction have more interaction with the electron density due to deeper or longer penetration into it which may cancel out much of the information about the incidence angles, especially seeing how very weak the trend is. No differences arise from varying the azimuth incidence angle.

In the adiabatic case (Tab. 3.22, the mean energy loss drops with growing polar incidence angle for total and differential ELD. This observation agrees with the findings of Kroes and coworkers [3, 4] who, using AIMD and $E_{\text {inc }}=5 \mathrm{eV}$, observed a decrease in energy loss and penetration probability as well as a increase of scattering probability if comparing scattering

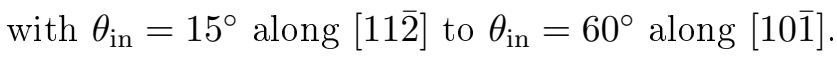

In the nonadiabatic case, the peak of the total ELD moves to higher energy losses with growing polar incidence angle and the intensity of the distribution's tail decreases, reflecting the lesser number of multibounce events. Fig. 3.41(a) illustrates this behavior: the shoulder of the total energy loss distribution moves from $E_{\text {loss }} \approx 1.4 \mathrm{eV}$ at low polar incidence angle to $E_{\text {loss }} \approx 0.9 \mathrm{eV}$ at large polar incidence angle, becoming more and more pronounced in the process due to a diminishing of the multibounce peak and the increase of the double-bounce peak in its stead (see Tab. 3.21, Fig. 3.41 and Fig. 3.42(b)-(h)). The double-bounce feature increases more strongly for the $[11 \overline{2}]$ then for the $[10 \overline{1}]$-direction. The insets in Fig. 3.41 and Fig. $3.42(\mathrm{~b})-(\mathrm{h})$ show that the main scattering intensity is in all cases along the direction of the incidence beam

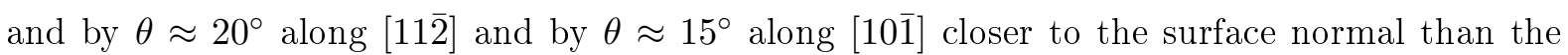
incidence polar angle. In all cases, the scattering in backwards direction is weak. This can also be seen from the angular distribution in incidence azimuth direction (Fig. 3.43 (e)); only the angular distribution for normal scattering is symmetric to the surface normal (black) and very clearly most intense at normal scattering; different from the other angular distributions it is also narrow. The $\phi$-resolved angular distributions of the other incidence angles behave very similarly in so far that they all peak close to the surface normal $\left(5-30^{\circ}\right)$ and show more intensity for 

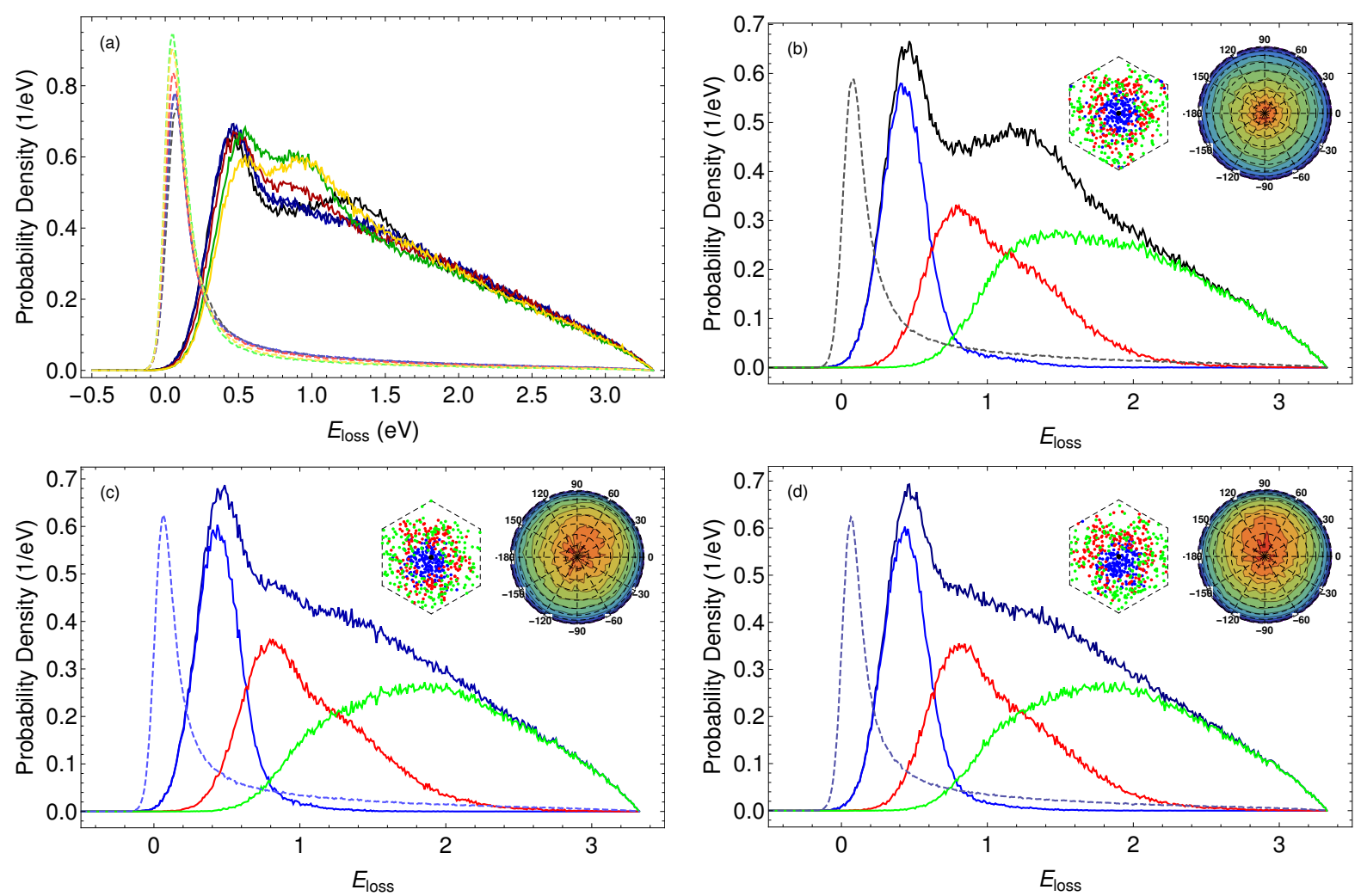

Figure 3.41.: Total energy loss distribution for different incidence angles. (a) for different incidence angles, the colors correspond those of the total energy loss distribution shown in (b)-(h) with the contributions from single (blue), double (red) and multibounce events (green); (b) $\theta_{\text {in }}=0^{\circ}$ (black), (c) $\theta_{\text {in }}=30^{\circ}, \phi_{\text {in }}=60^{\circ}$ (dark blue), (d) $\theta_{\text {in }}=30^{\circ}, \phi_{\text {in }}=90^{\circ}$ (navy). The light dashed distributions belong to adiabatic simulations, their intensity reduced by $0.2 \mathrm{eV}^{-1}$, the solid lines to nonadiabatic simulations. The inset shows the first impact site, reduced into the primitive cell, resolved after bounce events with 200 impact sites per bounce as well as the angular distribution where red marks the highest scattering $(>0.94)$ intensity and purple the weakest $(<1 / 12)$.

forward scattering than for backward scattering. This behavior is especially strong for large polar incidence angles and for the $[10 \overline{1}]$-incidence direction (green and red).

While at normal incidence, the $\mathrm{H}$ atoms are reflected at all regions of the primitive cell (Fig. 3.41(b) inset), the larger the polar angle grows, the more the region in forward direction is deprived of impacts, emphasizing how the rising hulk of the Au atom at very high polar angles shields the far side of the Au atoms from impacts (left inset in Fig. 3.41(b) vs those in Fig. $3.42 \mathrm{~kg}$ ) and $(\mathrm{h}))$. The distribution of the first impact site leading to the different bounce events follows the same pattern as previously remarked (see Sec.3.3.1 and Sec.3.3.2.

Figure 3.43(a) and (c) show the differential energy loss distribution for specular scattering; note that, due to the different incidence angles, the specular scattering angles also differ. At 

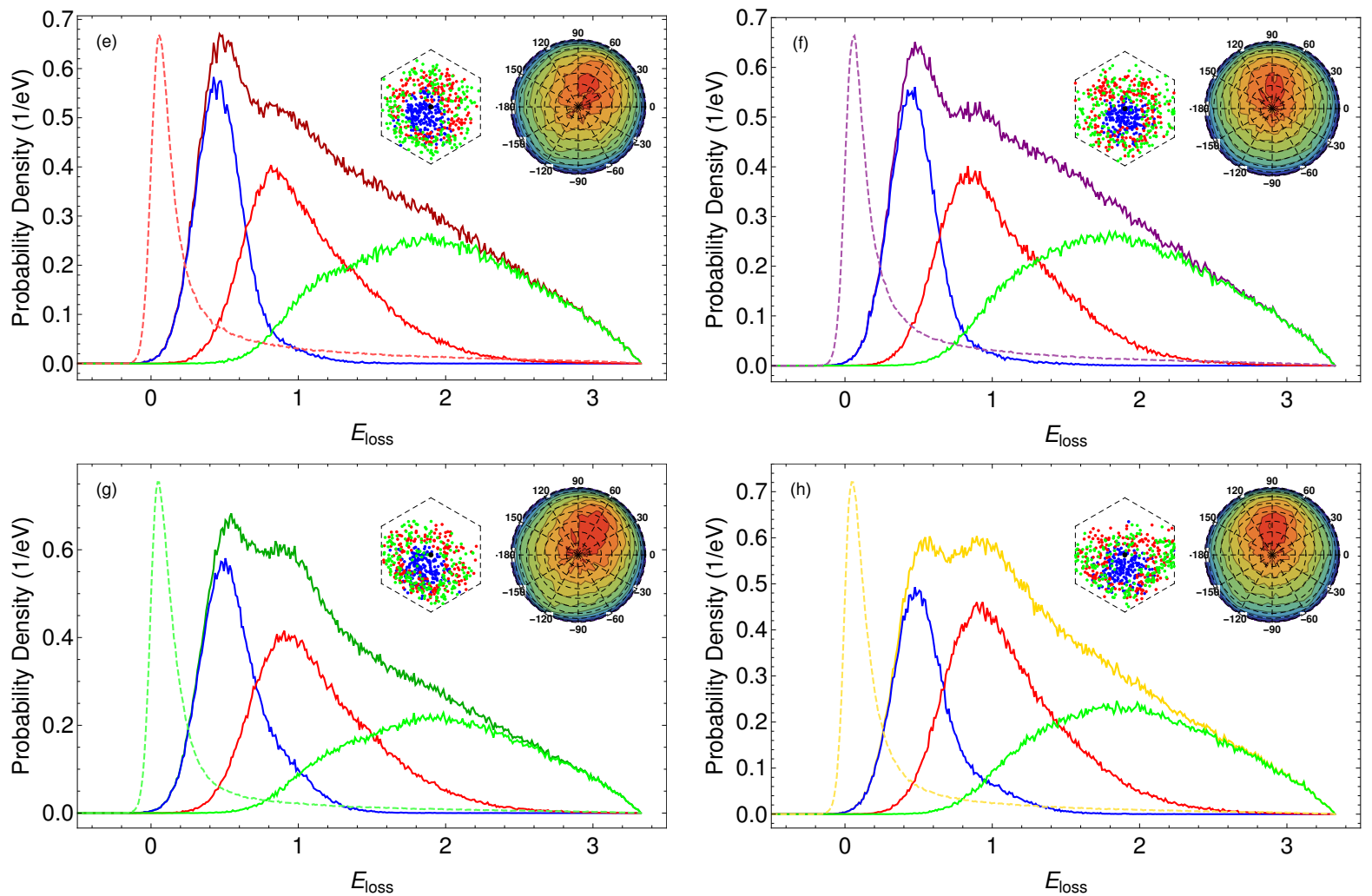

Figure 3.42.: Total energy loss distribution for different incidence angles with the contributions from single (blue), double (red) and multibounce events (green); (e) $\theta_{\text {in }}=45^{\circ}, \phi_{\text {in }}=60^{\circ}($ Dark Red), (f) $\theta_{\text {in }}=45^{\circ}, \phi_{\text {in }}=90^{\circ}$ (purple), (g) $\theta_{\text {in }}=60^{\circ}, \phi_{\text {in }}=60^{\circ}$ (dark green) (h) $\theta_{\text {in }}=$ $60^{\circ}, \phi_{\text {in }}=90^{\circ}$ (yellow). The light dashed distributions belong to adiabatic simulations, their intensity reduced by $0.2 \mathrm{eV}^{-1}$, the solid lines to nonadiabatic simulations. The inset shows the first impact site, reduced into the primitive cell, resolved after bounce events with 200 impact sites per bounce as well as the angular distribution where red marks the highest scattering $(>0.94)$ intensity and purple the weakest $(<1 / 12)$.

specular scattering for normal incidence (Fig. 3.43(a), (c), black), the peak due to multibounce events is stronger than the first peak. The intensity of the multibounce contribution is most likely due to the fact that many more $\mathrm{H}$ atoms penetrate the surface; as seen in Sec.3.3.1, these $\mathrm{H}$ atoms mainly undergo multibounce events and scatter predominantly normal to the surface. For the other differential ELDs, with growing polar incidence angle, the contribution due to the multibounce events diminishes slightly and the tail ends of the distributions flatten. As with the total ELD, with rising polar incidence angle the shoulder increases slightly, but its contribution is weaker than observed in the total ELD. Furthermore, the peak and rising edge of the differential distribution appear to move very slightly to higher energy losses the higher $\theta_{\text {in }}$ grows. 

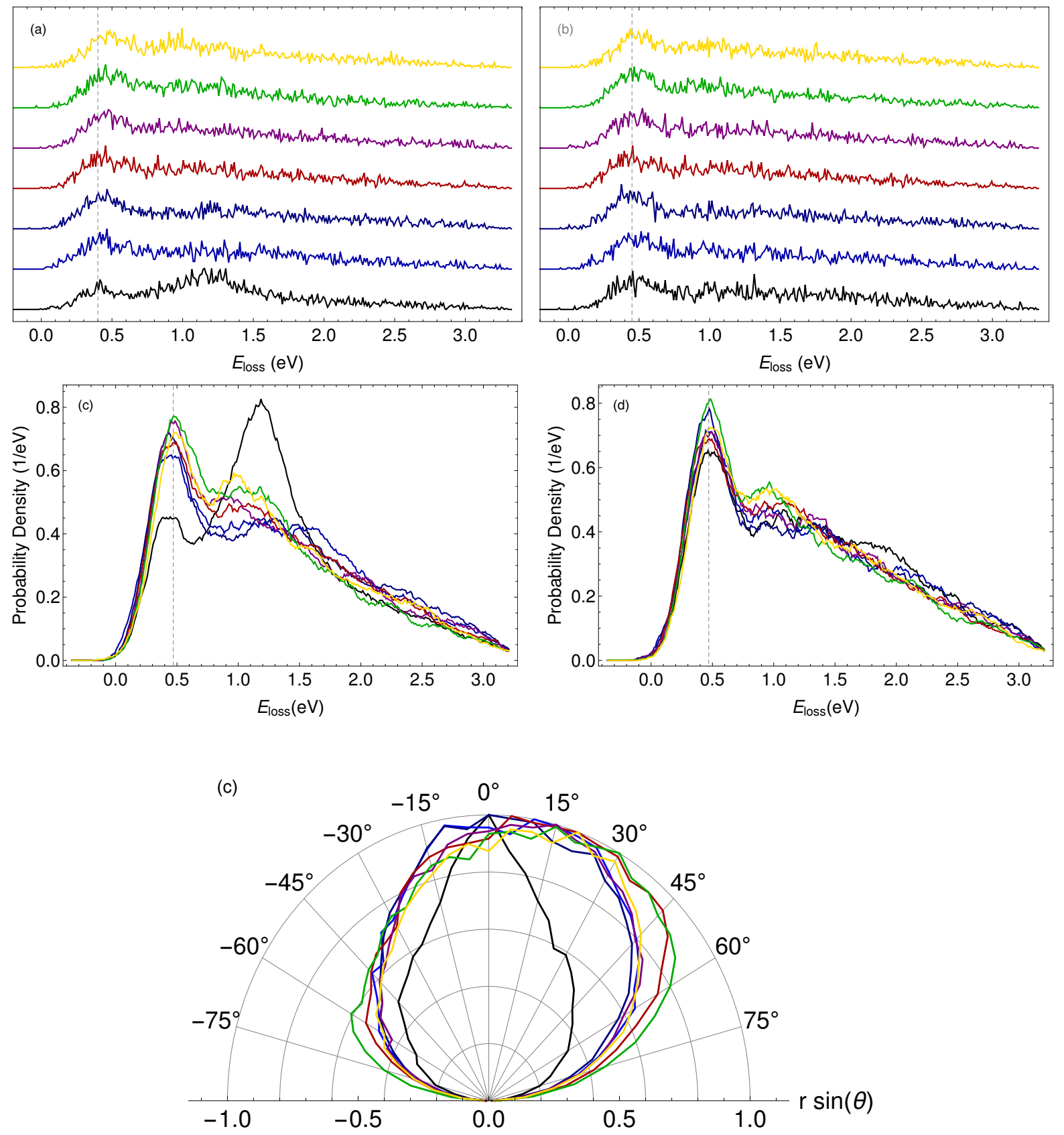

Figure 3.43.: Differential energy loss distribution for the incidence conditions: black: $\theta_{\text {in }}=0^{\circ}$, Dark Blue: $\theta_{\text {in }}=30^{\circ}, \phi_{\text {in }}=60^{\circ}$, Navy $\theta_{\text {in }}=30^{\circ}, \phi_{\text {in }}=90^{\circ}$, Darker Red: $\theta_{\text {in }}=45^{\circ}, \phi_{\text {in }}=60^{\circ}$, Purple: $\theta_{\text {in }}=45^{\circ}, \phi_{\text {in }}=90^{\circ}$, Darker Green: $\theta_{\text {in }}=60^{\circ}, \phi_{\text {in }}=60^{\circ}$, Yellow: $\theta_{\text {in }}=60^{\circ}$. (a) for specular scattering and (b) at $\theta_{\text {out }}=45^{\circ}$ and $\phi_{\text {out }}=60^{\circ}$ (c) for specular scattering (smoothed) and (d) at $\theta_{\text {out }}=45^{\circ}$ and $\phi_{\text {out }}=60^{\circ}$ (smoothed). The grey dashed line indicates the position of the first peak for the differential energy loss distribution of normal incidence and scattering, (e) Angular distribution in incidence azimuth direction, colors as described above. The positive $\theta$ denote the scattering angle $\theta_{\text {out }}$ in forward direction, the negative ones that in backward direction. 


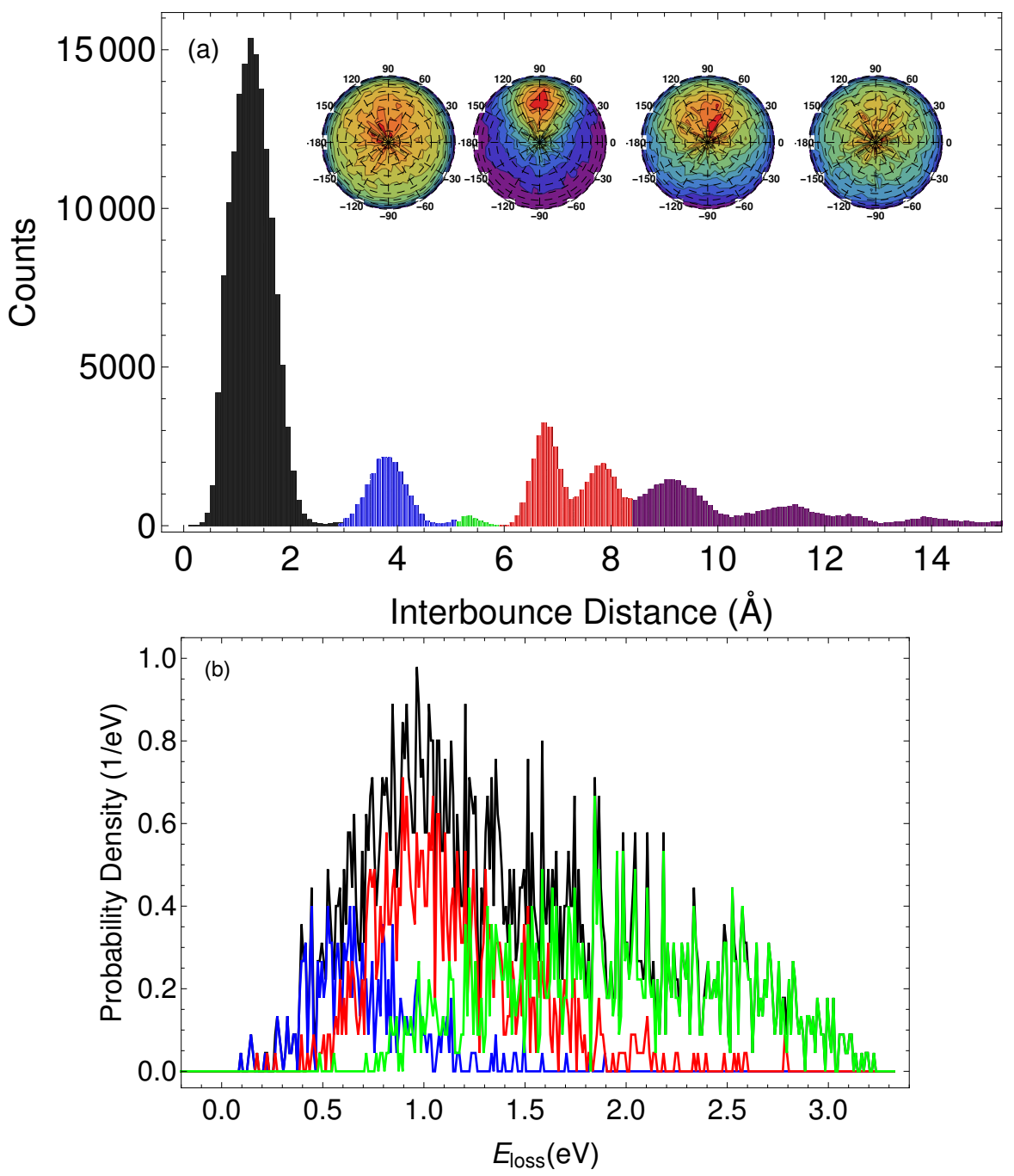

Figure 3.44.: (a)Analysis of double-bounce trajectories with respect to their second incidence site for $\theta_{\text {in }}=60^{\circ}$ and $\phi_{\text {in }}=90^{\circ}$ in a histogram showing the distance traveled between the $1^{\text {st }}$ and $2^{\text {nd }}$ bounces. The colors mark the collisions with different neighboring Au atoms: black: nearest neighbor, blue: next-nearest neighbor, green: next-next nearest neighbor, red: $3 \times$ next nearest neighbor, purple: all other neighbors. (b) differential energy loss distribution resolved into total (black), single-bounce (blue), double-bounce (red) and multibounce (green) events for the exit-angles $\theta_{\text {out }}=55^{\circ}$ and $\phi_{\text {in }}=-90^{\circ}$.

To compare the predictions made with MD simulations using EMT-JAWK for the scattering behavior at different incidence angles with experiment, the most promising incidence condition would be normal incidence as the simulations predict a strong shoulder that towers over the first peak of the differential energy loss distribution for specular scattering. However, to observe this particular scattering condition, the detector would have to be at the same position as the $\mathrm{H}$ atom beam source. Apart from perhaps the small shift of the peak of the differential energy loss distribution to higher energy losses with growing polar incidence angle, the form of the 
differential energy loss distributions of the other incidence angles at specular scattering does not show any distinct differences for the incidence conditions I have analyzed (Fig. 3.43). I thus analyzed the double-bounce events for $\theta_{\text {in }}=60^{\circ}, \phi_{\text {in }}=90^{\circ}$ as this is the incidence condition that has given rise to the strongest double-bounce contribution in the total energy loss distribution compared to the contributions due to other bounce events. It was therefore to be hoped that I could identify an exit condition where the double-bounce contribution would be particularly strong, resulting in a differential energy loss distribution that shows distinctly different features than differential energy loss distributions for other incidence angles which could be compared to experiment and seen whether the predictions for these regions would hold true.

The largest contribution to the double-bounce events $(62 \%)$ for $\theta_{\text {in }}=45^{\circ}, \phi_{\text {in }}=90^{\circ}$ are such that collide with the nearest neighbor. This contribution is slightly higher than noted for $\theta_{\text {in }}=45^{\circ}, \phi_{\text {in }}=60^{\circ}(58 \%$, see section 3.3.1), but in both cases, the $\mathrm{H}$ atoms bounce into all directions after their first impact and afterwards also scatter into all directions (see first inset in Fig. 3.44(a)). The collisions with the next-nearest neighbor atoms are much more specific in their scattering direction, but make up only $7 \%$ of all double-bounce events. All this makes the identification of a distinct region that is particularly strong in double-bounce events rather unlikely. The strongest contribution of double-bounce events over single-bounce events is to be had in back scattering direction at $\theta_{\text {out }}=55^{\circ}$ (which again is almost precisely backward scattering, Fig.3.44(b)). I could not identify any scattering condition where the second peak dominates the first as clearly as the total ELD suggests.

Fig. 3.43 (b), (d) show for an experimentally relevant scattering direction $\left(\theta_{\text {out }}=45^{\circ}, \theta_{\text {out }}=\right.$ $60^{\circ}$ ). While there are differences of the differential energy loss distributions for the different incidence conditions, these are not very strong: the peak of the differential ELDs remains roughly at the same energy losses and the overall form of the differential energy loss distributions also does not vary exceedingly (very close scrutiny will allow for a pusillanimously stronger shoulder

Table 3.23.: Exit angles under which high intensity can be expected for different incidence angles for the electronically nonadiabatic case.

\begin{tabular}{lll}
\hline \hline$\theta_{\text {in }}, \phi_{\text {in }}\left(^{\circ}\right)$ & $\theta_{\text {out }}\left(^{\circ}\right)$ & $\phi_{\text {out }}\left(^{\circ}\right)$ \\
\hline 0,0 & 0.0 & 0.0 \\
30,60 & $0-20$ & 60 \\
30,90 & $0-25$ & $60-150$ \\
45,60 & $0-40$ & $25-95$ \\
45,90 & & \\
60,60 & $0-45$ & $0-120$ \\
60,90 & $0-40$ & $40-140$ \\
\hline \hline
\end{tabular}


at high incidence polar angles). As already pointed out above, a comparison at $\theta_{\text {out }}=45^{\circ}$, $\theta_{\text {out }}=60^{\circ}$ does not only involve comparison of differential ELDs arising from different polar incidence angles, but also from forward (blue, red and green, Fig, 3.23(b) and (d)) and sideways (dark blue, purple and yellow) scattering. They cannot be distinguished from one another by the form of their differential ELD. As likewise mentioned above, the only quantity that differs slightly is the mean energy loss. It shows a slight decrease with growing polar incidence angle.

Tab 3.23 gives an overview where intense scattering can be expected to be found for the different incidence conditions.

I therefore expect that upon varying the incidence angle in experiment, no great differences between the differential energy loss distributions for different incidence angles will be observed.

\subsubsection{The Influence of Surface Structure on Scattering}

One of the drawbacks of studying $\mathrm{Au}(111)$ is that it is known to reconstruct, resulting in a large surface unit cell of $\sqrt{3} \times 22[32$, 33] or $\sqrt{3} \times 23$ [34, 35. So far, for MD calculations on $\mathrm{Au}(111)$, the surface reconstruction appears to have been ignored [3, 4] as the reconstruction requires a very large unit cell to be simulated. As will be seen in the next chapter (see Chapter 4), the MDsimulations performed with EMT-JAWK show some discrepancy to the experimental results. To give an estimate of whether surface reconstruction needs to be included into MD simulations and if its inclusion improves the comparison to the experimental results, I studied the effect of surface reconstruction on nonadiabatic scattering. For this, I used a $22 \times 6 \times 4$ slab and modeled the discommensuration lines according to the structures given by Wang et al. 44] and Hanke et al. [43]. Unlike the other calculations presented here, I used a four-layered slab to reduce the calculation time. To test the influence of small faults on the surface, I introduced one Au atom onto a $6 \times 6$ surface, leading to a coverage of $1 / 36$.

The scattering probability when surface reconstruction is included (Tab. 3.24) is only a little higher than for the unreconstructed surface. This can be attributed to structure of the recon-

Table 3.24.: Outcomes (in \%) resulting from $\mathrm{H}$ atom collision with a $\mathrm{Au}(111)$ surface for nonadiabatic and adiabatic (in parenthesis) simulations for different surface structures. The incidence energy is $E_{\text {inc }}=3.33 \mathrm{eV}, \theta_{\text {in }}=45^{\circ}$ along the [101] surface direction at $300 \mathrm{~K}$, number of simulated trajectories: nonadiabatic: $10^{6}$, adiabatic: $10^{5}$.

\begin{tabular}{lllll}
\hline \hline structure & Scattering & $\begin{array}{l}\text { Surface } \\
\text { Adsorption }\end{array}$ & $\begin{array}{l}\text { Subsurface } \\
\text { Absorption }\end{array}$ & Transmission \\
\hline relaxed & $55(81)$ & $23(4)$ & $21(7)$ & $1(8)$ \\
1 adatom & 56 & 22 & 22 & 1 \\
reconst. & $59(80)$ & $15(2)$ & $23(5)$ & $0(13)$ \\
\hline \hline
\end{tabular}


Table 3.25.: Outcomes (\%) of scattering for various scattering events resulting from $\mathrm{H}$ atom collision with a $\mathrm{Au}(111)$ surface for nonadiabatic and adiabatic (in parenthesis) simulations for different surface structures. The "Surface"-column refers to trajectories wherein the H atoms scattered from $1^{\text {st }}$ layer of the surface. The Roman numerals refer to the lowest subsurface to which penetration occurred. The incidence conditions are $E_{\text {inc }}=3.33 \mathrm{eV}$, $\theta_{\text {inc }}=45^{\circ}$ along the $[10 \overline{1}]$ surface direction and $6 \times 6 \times 6$ cell at $300 \mathrm{~K}$, number of simulated trajectories: nonadiabatic: $10^{6}$, adiabatic: $10^{5}$.

\begin{tabular}{lllllllll}
\hline \hline & \multicolumn{3}{l}{ bounce events } & & \multicolumn{5}{c}{ penetrating bounces } \\
structure & single & double & multi & surface & I & II & III & $>$ III \\
\hline relaxed & $23(17)$ & $34(25)$ & $43(59)$ & $82(64)$ & $17(23)$ & $1(8)$ & $0(3)$ & $0(2)$ \\
1 adatom & 22 & 33 & 45 & 83 & 15 & 1 & 0 & 0 \\
reconst. & $24(19)$ & $37(29)$ & $39(52)$ & $88(74)$ & $11(17)$ & $1(7)$ & $0(3)$ & $0(0)$ \\
\hline \hline
\end{tabular}

structed surface (see Fig.2.1.(c) and (d)) that is closer than the unreconstructed surface, making it more difficult for an $\mathrm{H}$ atom to go subsurface or to resurface again which agrees well with the higher percentage of non-penetrating trajectories (Tab. 3.25). The probability to remain at the surface is lower than for the unreconstructed case, most probably because the way to populate the surface is to a large part via resurfacing. This is more difficult if the surface is packed denser and overlaps subsurface wells in some regions. The absorption and transmission probability is higher than for the relaxed surface. Although it could be expected that the absorption and transmission probability should be lower for a reconstructed surface, the higher ratio can be explained by the previous observation: fewer $\mathrm{H}$ atoms might be able to penetrated, but if the $\mathrm{H}$ atoms are hindered from resurfacing again due to the reconstruction on the surface, then the number of atoms that remain subsurface or are transmitted can well be higher than in the

Table 3.26.: Energy loss in \% of incidence energy for various outcomes resulting from $\mathrm{H}$ atom collision with a $\mathrm{Au}(111)$ surface for nonadiabatic and adiabatic (in parenthesis) simulations for different surface structures. The mean and maximum energy loss are shown for the total and differential ELD. Number of simulated trajectories: nonadiabatic: $10^{6}$, adiabatic: $10^{5}$. The accuracy for specular scattering $\left(\theta_{\text {out }}=45^{\circ} \phi_{\text {out }}=60^{\circ}([10 \overline{1}])\right)$ has been reduced to account for the lower signal-to-noise ratio in the differential ELD.

\begin{tabular}{lllll}
\hline \hline & Total & & \multicolumn{2}{l}{$\theta_{\text {out }}=45^{\circ} \phi_{\text {out }}=60^{\circ}$} \\
structure & Mean & Peak & Mean & Peak \\
\hline relaxed & $35.3(4.65)$ & $14.0(1.95)$ & $39(12)$ & $14(0.75)$ \\
1 adatom & 35.6 & 14.3 & 39 & 15 \\
reconst. & $32.9(4.05)$ & $15.2(2.25)$ & $35(8.7)$ & $15(1.1)$ \\
\hline \hline
\end{tabular}



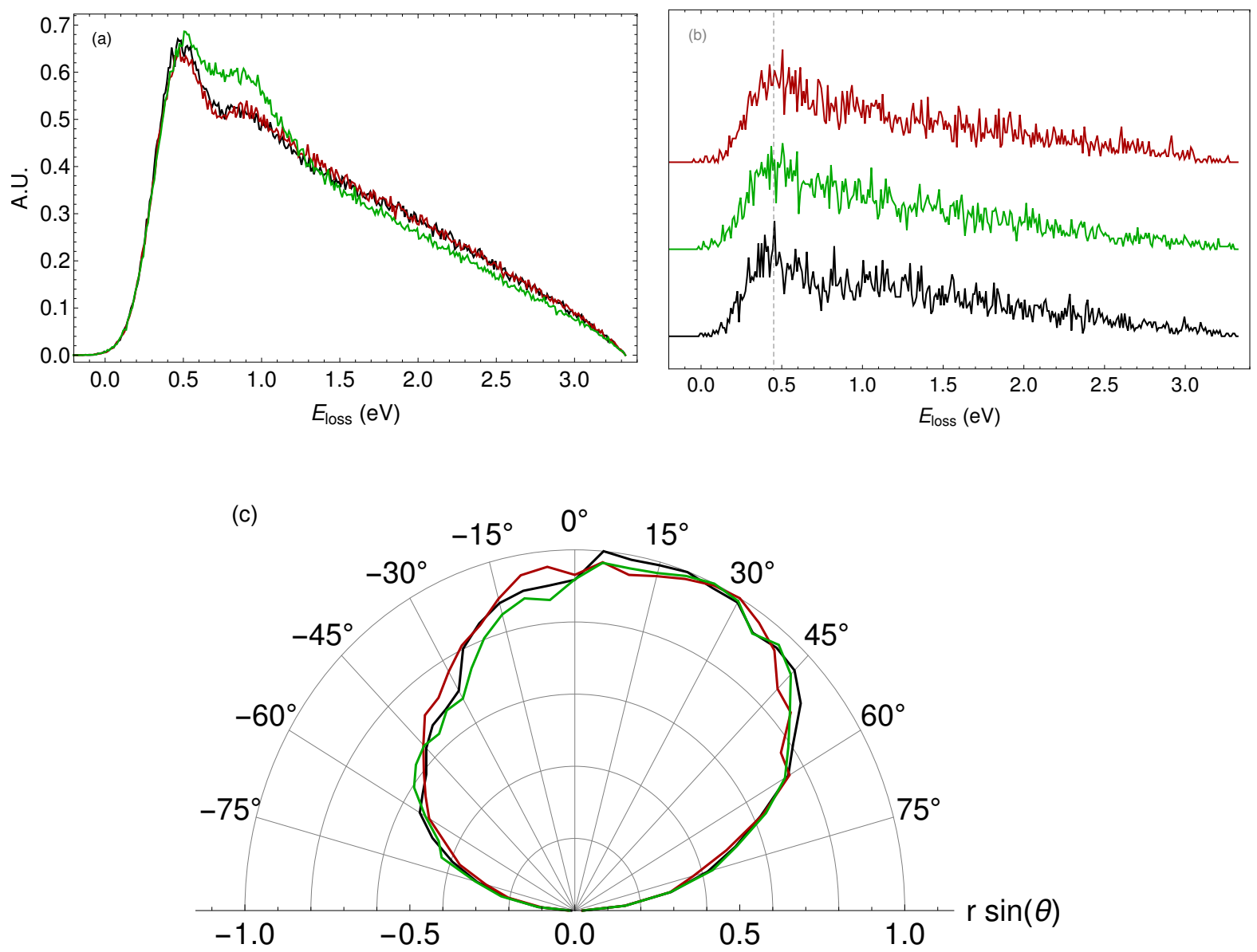

Figure 3.45.: Energy loss distribution for (a) total and (b) specular scattering $\left(\theta_{\text {out }}=45^{\circ}\right.$ and $\phi_{\text {out }}=$ $60^{\circ}$ ) for the unreconstructed surface (black), the reconstructed surface (dark green) and the unreconstructed surface with an adatom (dark red). (c) Angular distribution for scattering along the [101] -direction. Negative $\theta_{\text {out }}$ correspond to backward scattering, positive ones to forward scattering. A cosine distribution is given in navy.

relaxed case. The ratio of the bounce events stays roughly the same, but the peak of the total energy loss distribution for the reconstructed surface is shifted to higher energy losses and the shoulder stronger (Fig. 3.45(a), green) while the mean energy loss is slightly lower than for the unreconstructed surface (see also Tab. 3.26.

Having an adatom on the surface does not change the dynamics much, neither with respect to reflection nor with respect to ab- or adsorption (Tab. 3.24) or ratio of bounce events (Tab. 3.25). The form of the energy loss distribution and its energy losses remain almost the same.

The angular distribution of scattering along the [101] -direction is for all three considered surfaces much the same (Fig.3.45(c)). They are all equally broad and peak at she same outgoing angles, showing barely any difference amongst one another, although the unreconstructed surface has a peak at $\theta_{\text {out }} \sim 5^{\circ}$, the unreconstructed, reconstructed and surface with adatom all show 
Table 3.27.: Outcomes (in \%) resulting from $\mathrm{H}$ and D atom collisions with a $\mathrm{Au}(111)$ surface for nonadiabatic and adiabatic (in parenthesis) simulations. The incidence conditions are $\theta_{\text {inc }}=45^{\circ}$ along the $[10 \overline{1}]$ surface direction, $T=300 \mathrm{~K}$ with $6 \times 6 \times 6$ slab, number of simulated trajectories: $10^{6}$. For deuterium and hydrogen an incidence energy of $3.27 \mathrm{eV}$ and $3.33 \mathrm{eV}$, respectively, was used.

\begin{tabular}{lllll}
\hline & Scattering & $\begin{array}{l}\text { Surface } \\
\text { Adsorption }\end{array}$ & $\begin{array}{l}\text { Subsurface } \\
\text { Absorption }\end{array}$ & Transmission \\
\hline \hline $\mathrm{H}$ & $55(82)$ & $23(4)$ & $21(6)$ & $1(8)$ \\
$\mathrm{D}$ & $58(74)$ & $21(8)$ & $20(13)$ & $1(5)$ \\
\hline \hline
\end{tabular}

a peak at $\theta_{\text {out }} \sim 25^{\circ}$. The comparison of specular scattering (Fig. 3.45 (b)) does not reveal large differences between the surface types: the first peak of the differential energy loss distributions is dominant for all surfaces and although the tail of the differential energy loss distribution of specular scattering for the reconstructed surface (green) is a bit more filled out than for the unreconstructed surface (black), the increase of a shoulder close to the first peak is barely visible. The addition of an adatom (dark red) to the surface does not result in any remarkable changes of the differential energy loss distribution at specular scattering, either.

It appears therefore that, short of a very severe deviation from the (111) surface structure (perhaps by changing the crystal facet), the surface structure has little influence on the scattering energy loss distribution.

\subsubsection{Isotope effect}

Hydrogen is a factor of two lighter than deuterium; possessing the same incidence energy, $\mathrm{H}$ therefore has a $\sqrt{2}$ higher impact velocity than D. To study the isotope effect, I performed MD simulations for deuterium and hydrogen with $E_{\text {inc,D }}=3.27 \mathrm{eV}$ and $E_{\mathrm{inc}, \mathrm{H}}=3.33 \mathrm{eV}$, respectively which match the incidence energy values that have been used in experiment.

Tab. 3.27 shows the different event probabilities for the calculations that are to be compared to experiment. Only the adiabatic case shows larger discrepancies: the scattering and transmission probability of $\mathrm{D}$ is lower than for $\mathrm{H}$ while the ad- and absorption probability is higher although the penetrating probability for deuterium (see Tab. 3.21) is lower than for hydrogen. The ratio of the different bounce events shows a slight preference for multibounce events in case of hydrogen compared to deuterium. In the nonadiabatic case, the probabilities of scattering, adsorption, penetration and the ratio between the bounce events (see Tab. 3.27 and Tab.3.21 do not show any remarkable difference, in accordance with both experiencing the same friction coefficient $\eta(r)$ at a given position $r$.

Tab. 3.29 shows that the adiabatic mean energy loss of hydrogen compared to deuterium 
Table 3.28.: Outcomes (\%) for various scattering events resulting from H and D atom collisions with a $\mathrm{Au}(111)$ surface for nonadiabatic and adiabatic (in parenthesis) simulations. The "Surface"column refers to trajectories wherein $\mathrm{H}$ atoms scattered from the first layer of the surface. The Roman numerals refer to the lowest subsurface to which penetration occurred. The incidence conditions are $\theta_{\text {inc }}=45^{\circ}$ along the $[10 \overline{1}]$ surface direction, $T=300 \mathrm{~K}$ with $6 \times 6 \times 6$ cell, number of simulated trajectories: $10^{6}$. For deuterium and hydrogen an incidence energy of $3.27 \mathrm{eV}$ and $3.33 \mathrm{eV}$, respectively, was used.

\begin{tabular}{llllllllll}
\hline \hline & \multicolumn{3}{c}{ bounce events } & \multicolumn{5}{c}{ penetrating bounces } \\
Isotope & single & double & multi & surface & I & II & III & > III \\
\hline $\mathrm{H}$ & $23(17)$ & $34(25)$ & $43(59)$ & $82(64)$ & $17(23)$ & $1(8)$ & $0(3)$ & $0(2)$ \\
$\mathrm{D}$ & $22(18)$ & $33(27)$ & $45(55)$ & $83(70)$ & $16(23)$ & $1(5)$ & $0(1)$ & $0(1)$ \\
\hline \hline
\end{tabular}

is lower while the inclusion of energy loss to electron hole pair excitation shows the reverse behavior, i.e. the nonadiabatic mean energy loss for the hydrogen is larger than that of the $\mathrm{D}$ atom. This is also evident from Fig. 3.46(a) and (b). The total energy loss distribution of deuterium (Fig. 3.46(a)) has a slightly different shape than that of $\mathrm{H}$ due to slightly differently shaped total energy loss distributions of the individual bounce events. This difference in shape does not carry into the differential energy loss distributions at specular angles (see Fig. 3.46.(b)). Here, the differential energy loss distributions of both isotopes show an almost perfect overlap with one another. The angular distribution along the [101] -direction also does not show any different behavior: both that for $\mathrm{D}$ and for $\mathrm{H}$ are very broad, almost identically formed, showing mostly scattering in forward direction and their peak positions agree well (see Fig.3.46(c)).

To further explore the isotope effect, I performed calculations for 1000 trajectories for both

Table 3.29.: Energy loss in \% of incidence energy for various outcomes resulting from $\mathrm{H}$ and $\mathrm{D}$ atom collisions with a $\mathrm{Au}(111)$ surface for nonadiabatic and adiabatic (in parenthesis) simulations. The incidence conditions are $\theta_{\mathrm{inc}}=45^{\circ}$ along the [101] surface direction, $T=300 \mathrm{~K}$ with $6 \times 6 \times 6$ cell, number of simulated trajectories: $10^{6}$. For deuterium and hydrogen an incidence energy of $3.27 \mathrm{eV}$ and $3.33 \mathrm{eV}$, respectively, was used. The mean and maximum energy loss are shown for the total and differential ELD. The reduced accuracy at specular scattering $\left(\theta_{\text {out }}=45^{\circ} \phi_{\text {out }}=60^{\circ}([10 \overline{1}])\right)$ is due to the lower signal-to-noise ratio in the differential ELDs.

\begin{tabular}{lllll}
\hline \hline & \multicolumn{2}{l}{ Total } & & \multicolumn{2}{l}{$\theta_{\text {out }}=45^{\circ} \phi_{\text {out }}=60^{\circ}$} \\
Isotope & Mean & Peak & Mean & Peak \\
\hline H & $39.2(13.1)$ & $14.0(1.65)$ & $37(11)$ & $14(0.75)$ \\
D & $36.1(16.7)$ & $12.7(2.91)$ & $33(14)$ & $12(2.6)$ \\
\hline \hline
\end{tabular}


isotopes with the same incidence energy of $3.31 \mathrm{eV}$ for normal incidence at $T=0 \mathrm{~K}$ for the electronically adiabatic and nonadiabatic case. To separate the contribution due to the adiabatic and nonadiabatic energy loss, I also performed an MD simulation at the above conditions where I keep the Au atoms fixed at their equilibrium positions of the relaxed slab (MDEF-SS). For all these calculations, I compare the positions of the peaks of the total energy loss distribution due to the single-bounce events for hydrogen with those of deuterium.

In the electronically adiabatic case, when the energy loss to ehp is switched off, the total energy loss distribution due to single-bounce events of hydrogen peaks at $55 \mathrm{meV}$ and that of the deuterium at $\sim 2$ times higher energy losses of $105 \mathrm{meV}$ (Fig.3.47, blue), resulting in an energy difference of $50 \mathrm{meV}$. The comparison of the positions of the peaks for $\mathrm{H}$ and $\mathrm{D}$ for the MDEF-SS calculations with the solid surface peak at $255 \mathrm{meV}$ for the deuterium atom and at
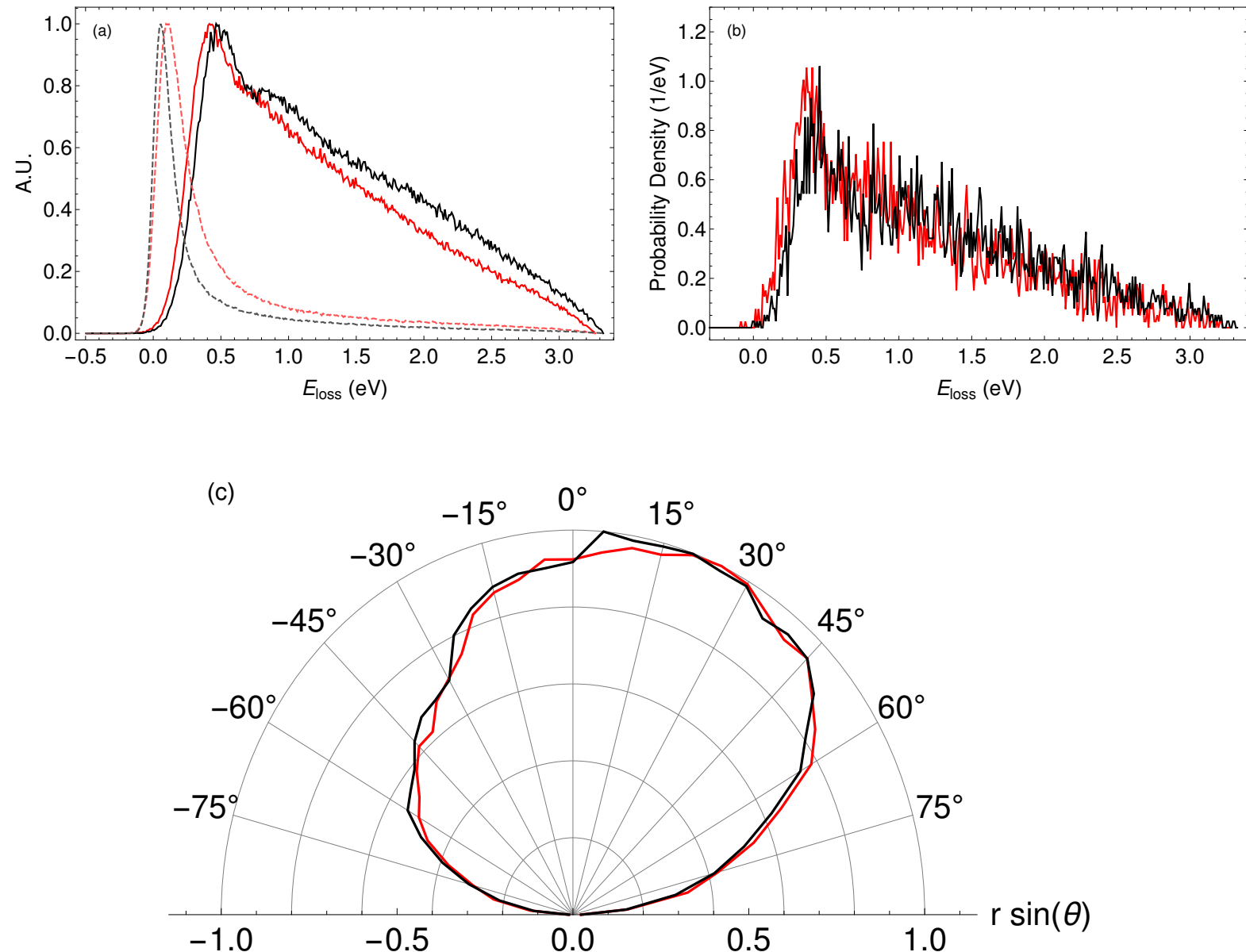

Figure 3.46.: Energy loss distribution for nonadiabatic scattering of $\mathrm{H}$ (black, solid) and D (red, solid) and the electronically adiabatic scattering results (light, dashed), (a) total, (b) specular scattering $\left(\theta_{\text {out }}=45^{\circ}, \phi_{\text {out }}=60^{\circ}\right)$. (c) Angular distribution of scattering in [101] direction for $\mathrm{H}$ (black) and $\mathrm{D}$ (red). $\theta$ is the polar angle and negative values correspond to scattering in backward direction. 


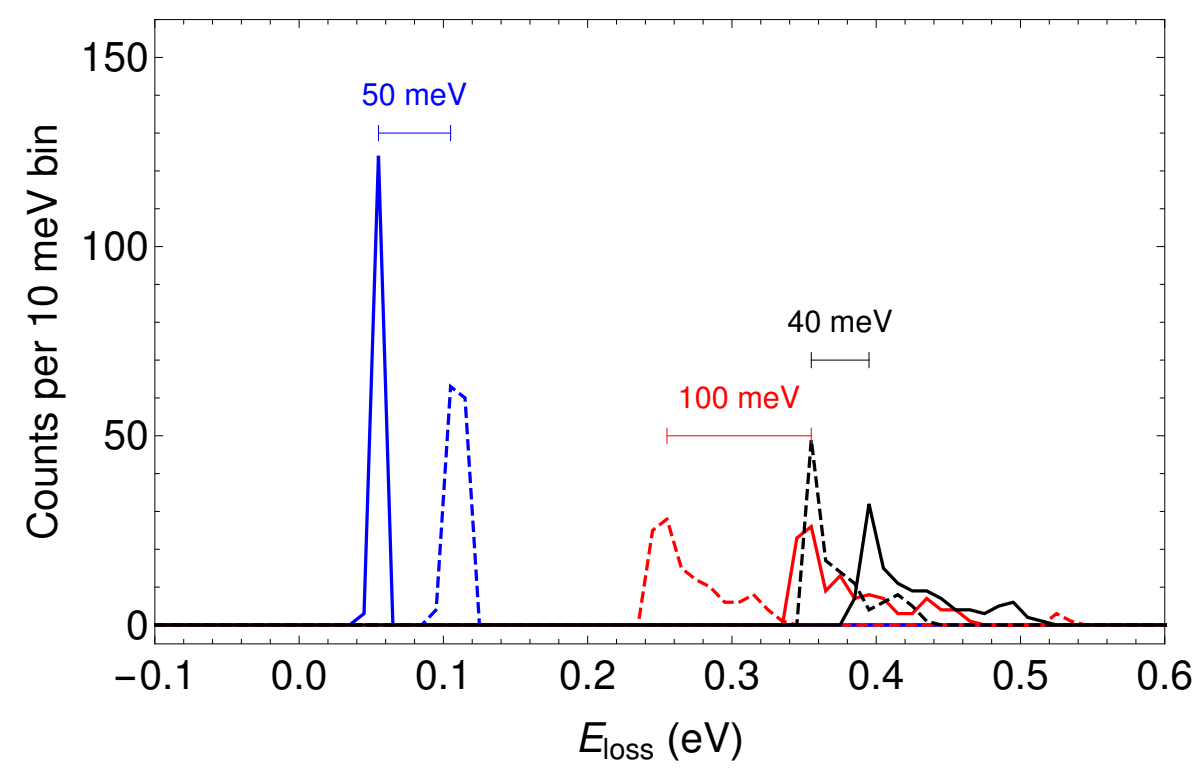

Figure 3.47.: Total energy loss distribution due to single-bounce events for different isotopes (H solid, D dashed) at $E_{\text {inc }}=3.31 \mathrm{eV}$ electronically adiabtic at $0 \mathrm{~K}$ (blue), electronically nonadiabatic with frozen surface (red) and nonadiabatic at $0 \mathrm{~K}$ (black).

$\sim \sqrt{2}$ higher energy losses of $355 \mathrm{meV}$ for the $\mathrm{H}$ atom, resulting in an difference in energy loss to ehp of $100 \mathrm{meV}$ (Fig. 3.47, red). It should be noted that the energy loss to electron hole pairs clearly is much larger than that to phonons for both hydrogen and deuterium.

If the electronically nonadiabatic case with a surface temperature of $0 \mathrm{~K}$ is considered (that is, if both adiabatic and nonadiabatic effects are considered together), the difference in energy loss amounts to $40 \mathrm{meV}$ (Fig. 3.47, black).

The adiabatic energy loss can be estimated by means of the Baule limit which can be represented in terms of the projectile-surface mass ratio $\mu=m / M$ :

$$
\Delta E_{\text {ad }}=\frac{4 \mu}{(1+\mu)^{2}} E_{\text {inc }}
$$

It follows that for the same incidence energy

$$
\frac{\Delta E_{\mathrm{ad}}(\mathrm{D})}{\Delta E_{\mathrm{ad}}(\mathrm{H})}=\frac{4 \mu_{D}}{\left(1+\mu_{D}\right)^{2}} E_{\mathrm{inc}}: \frac{4 \mu_{H}}{\left(1+\mu_{H}\right)^{2}} E_{\mathrm{inc}} \approx \frac{m_{D}}{m_{H}}=2 .
$$

The nonadiabatic energy loss due to friction is given by the integral

$$
\Delta E_{\mathrm{na}}=\int \eta(\mathbf{r}) \mathbf{v} \cdot d \mathbf{s}
$$

along the entire trajectory. Here, $\mathbf{v}$ is the particle's velocity and $\mathbf{s}$ the distance along the trajectory. Assuming the friction coefficient to be independent on the particle's position, the ratio of the nonadiabatic energy loss $\Delta E_{\text {na }}$ for deuterium and hydrogen can be estimated to be proportional to their velocities $v$ :

$$
\frac{\Delta E_{\mathrm{na}}(\mathrm{D})}{\Delta E_{\mathrm{na}}(\mathrm{H})} \approx \frac{\eta v_{\mathrm{D}}}{\eta v_{\mathrm{H}}} \approx \frac{v_{\mathrm{D}}}{v_{\mathrm{H}}} \approx \frac{\sqrt{2 E_{\mathrm{inc}} / m_{\mathrm{D}}}}{\sqrt{2 E_{\mathrm{inc}} / m_{\mathrm{H}}}}=\sqrt{\frac{m_{\mathrm{H}}}{m_{\mathrm{D}}}}=\frac{1}{\sqrt{2}}
$$


One would therefore expect that deuterium should experience a factor of $\sqrt{2}$ lower energy loss than hydrogen at the same incidence energy. This effect is opposite to that for the adiabatic collisions which becomes clear if Eq. 3.2 is compared to Eq. (3.4).

In conclusion, the isotope effect on the energy loss to phonons and to electron hole pair operates into opposite directions in the simulations, leading to almost a compensation for $\mathrm{H}$ and $\mathrm{D}$ whose energy loss behavior is therefore very similar.

\subsubsection{The Influence of Changes in Electron Density}

The coupling of electronic degrees of freedom to nuclear ones is taken into account by means of LDFA (see section 2.5 in this work. The friction coefficient used in the resulting Langevin equations is related to the background electron density (Eq.2.30). In the derivation of this formula some approximations were employed. Here, I investigated how the electronically nonadiabatic adsorption dynamics are influenced by changes in the background electron density, perhaps offering a possible explanation on the divergences to the experimental results (see chapter,4). I followed four different approaches:

In the first case, I lower the electron background density to electron density from $a b$ initio simulations (see section 3.2 and Fig. 3.48). To this end, I multiplied the background electron density the $\mathrm{H}$ atom experiences throughout the trajectory by a factor of $2 / 3$. Fig. 3.48 shows how the reduction of the electron background density of EMT (blue) by a factor of $2 / 3$ (green) reproduces the electron density from the GGA-DFT calculations (grey) [22] for the top-site. Fig. 3.49 (a), (b) shows the comparison of the ELD resulting from the unmodified background electron density (black) to the ELDs resulting from the modifications of the background electron density. The consequence of lowing the background electron by $2 / 3$ (green) is that the peak and shoulder of the (total and differential) ELD shift to slightly lower energy losses. The scattering and adsorption dynamics (see Tab. 3.30, (1)) change slightly towards more scattering and less adsorption while the percentage of multibounce events increases, in accordance with the slightly (due to the shift of the first peak) longer tail of the ELD (Tab.3.31. (1)). The shift between the two ELDs is however not large (Tab.3.32, (1)), indicating that a small modification to the electron density is of little consequence.

For the second case, I assume that the background electron density can be described by an analytic expression given by a logistic equation

$$
n(z)=\frac{0.25}{1+\exp [6.0(z-1.7)]},
$$

where $z$ is the $\mathrm{H}$ atom distance normal to the surface. The coefficients in Eq. (3.5) are chosen such that the electron density decays at the surface in agreement with the EMT background electron density. It assumes a constant value within the slab and a constant one outside (Fig. 3.50). The total energy loss distribution obtained when applying Eq. (3.5) (Fig 3.49(a), red) to calculate 


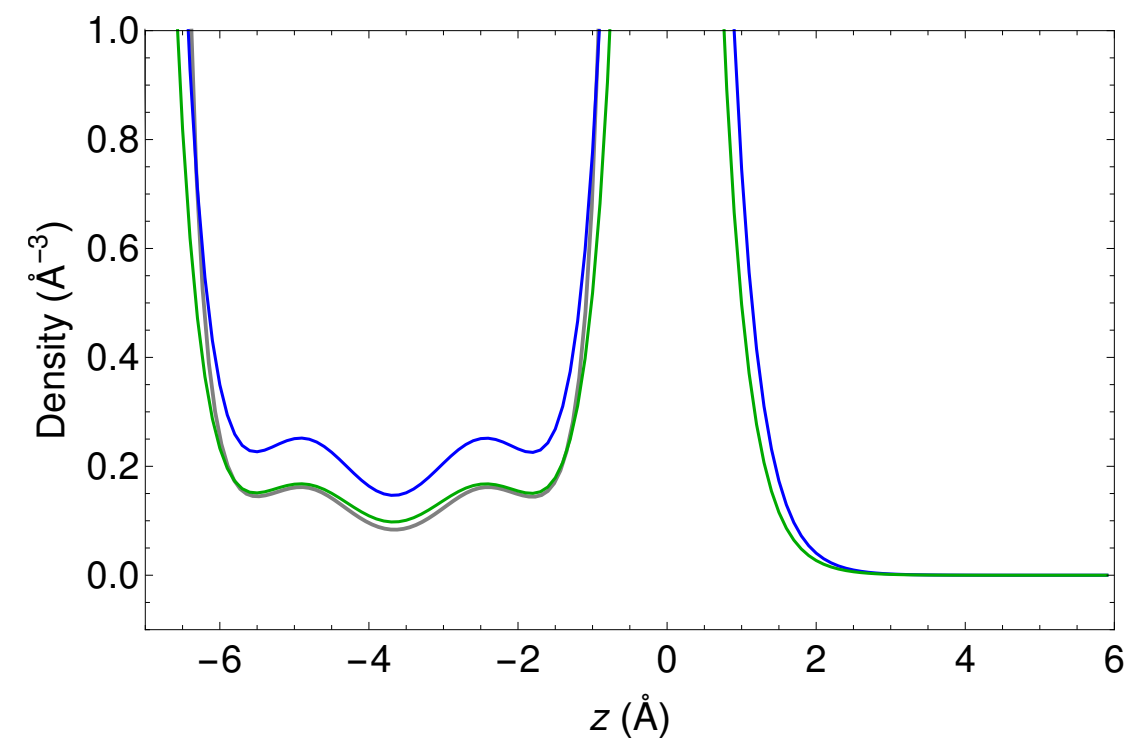

Figure 3.48.: The EMT background electron density (blue) of an $\mathrm{H}$ atom and the one reduced by $2 / 3$ (green) compared to the density of the GGA-DFT calculations (grey) for the top-site.

the background electron density peaks at higher energy loss and its form changes significantly compared to the total ELD calculated with the unmodified background electron density (black) in that the shoulder resulting from double-bounce events grows more pronounced and a new shoulder appears at high energy losses resulting from a large overlap between the total energy loss distribution of double- and multibounce events. The sticking and ad/absorption behavior changes slightly towards less scattering and more sticking (Tab.3.30) and the ratio of bounce events is more evenly split up (Tab. 3.31). The differential energy loss distribution at specular scattering (Fig 3.49(b), red) is likewise shifted to higher energy losses and shows a slightly fuller tail. The angular distribution in $[10 \overline{1}]$-direction is only insignificantly changed; what changes

Table 3.30.: Outcomes (in \%) resulting from $\mathrm{H}$ atom collision with a $\mathrm{Au}(111)$ surface for nonadiabatic simulations for modifications to the background electron density. The incidence conditions are $E_{\text {inc }}=3.33 \mathrm{eV}, \theta_{\mathrm{inc}}=45^{\circ}$ along the $[10 \overline{1}]$ surface direction, with a relaxed surface structure and $6 \times 6 \times 6$ slab at $300 \mathrm{~K}$, number of simulated trajectories: $10^{6}$.

\begin{tabular}{lllll}
\hline \hline Modification & Scattering & $\begin{array}{l}\text { Surface } \\
\text { Adsorption }\end{array}$ & $\begin{array}{l}\text { Subsurface } \\
\text { Absorption }\end{array}$ & Transmission \\
\hline none & 55 & 23 & 21 & 1 \\
$(1)$ & 59 & 20 & 21 & 1 \\
$(2)$ & 51 & 27 & 22 & 0 \\
$(3)$ & 55 & 23 & 21 & 1 \\
$(4)$ & 82 & 4 & 6 & 8 \\
\hline \hline
\end{tabular}



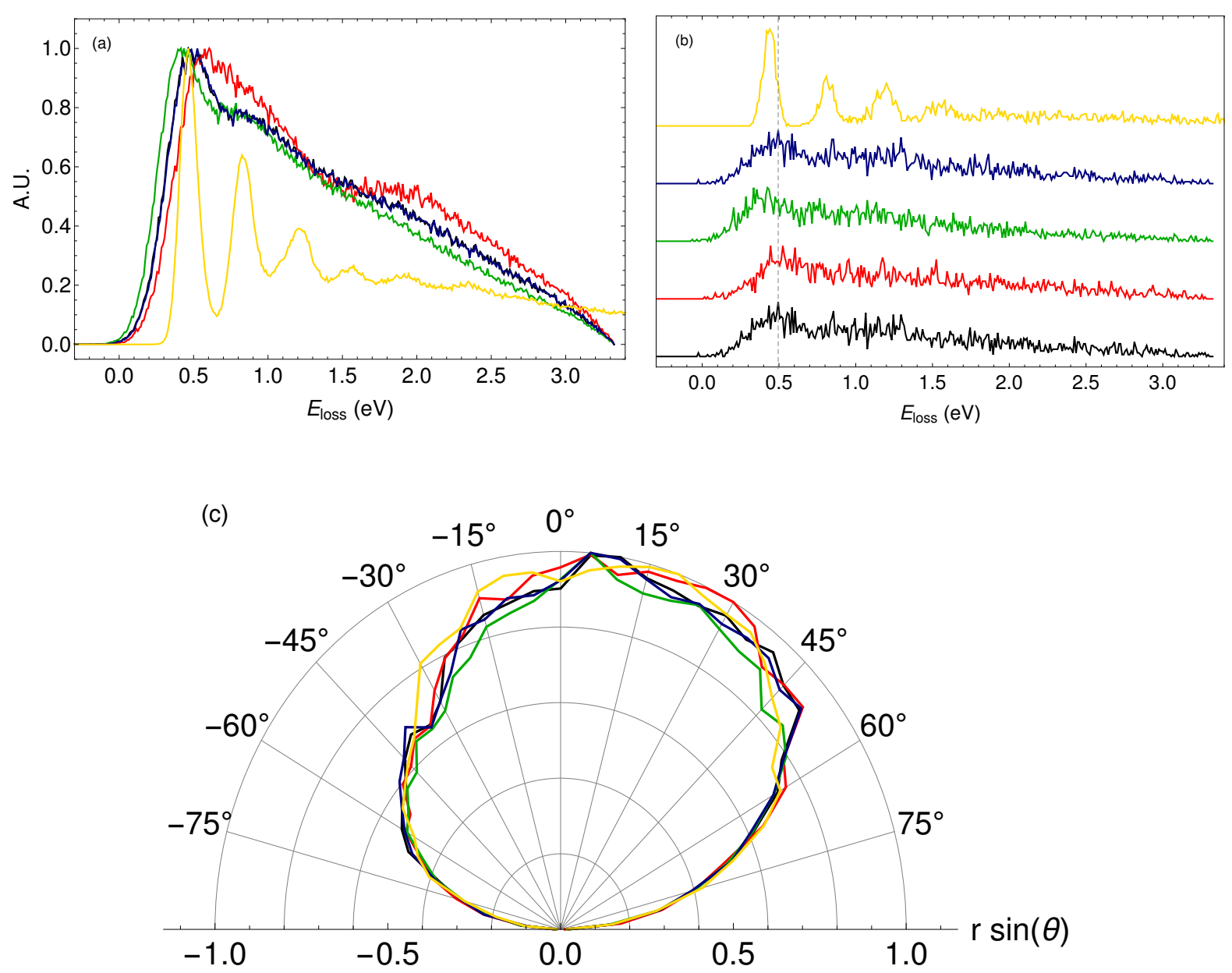

Figure 3.49.: (a) Total energy loss distribution of nonadiabatic calculation for different modifications to the electron density. (b) differential energy loss distribution at specular scattering angle. The dashed line indicates the position of the peak of the differential energy loss distribution resulting from the unmodified EMT electron density (black). (c) Angular distributions along the [101] -direction, negative $\theta$ correspond to backwards scattering. Unmodified background electron density (black), constant density in surface (red), electron density reduced by factor of $2 / 3$ (green), density increased at repulsive walls (navy) and electronic friction post facto (yellow).

are to be observed are more likely to be caused by noise than any fundamental difference due to the modification of the background electron density (Fig. 3.49(c), red). It is almost surprising that such a large modification in electron density does not result in a more significant change in the ELDs. It is an indication that the region of electron density the $\mathrm{H}$ atom moves in or scatters at sees no great variations and that the $\mathrm{H}$ atom does not come close enough to the atom cores to experience regions of very high electron density.

The latter point is especially emphasized (case 3) when the background electron density is increased when the $\mathrm{H}$ atom comes close to the Au atoms. In this way, while an atom would 


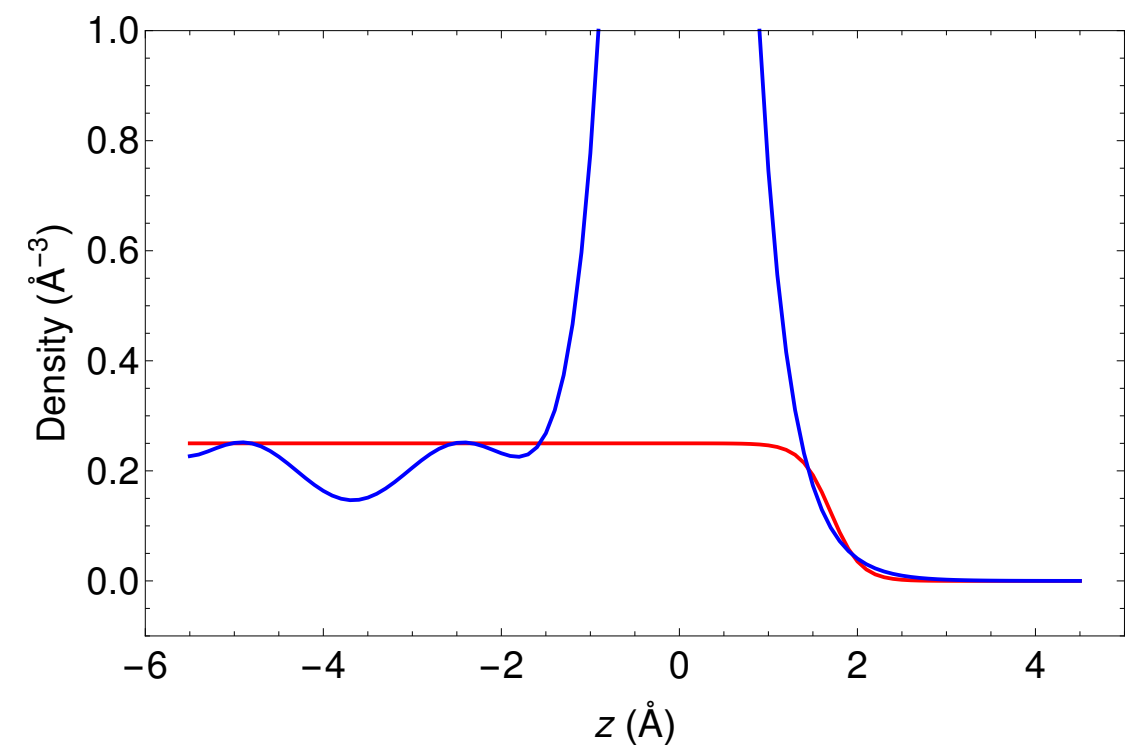

Figure 3.50.: The EMT background electron density (blue) of an $\mathrm{H}$ atom and the one given by Eq. 3.5 (red), for the top-site.

experience higher electron density in a direct collision with the Au atoms, an atom ad- or absorbed to the surface experiences the same background electron density as directed by the EMT-parameters. To achieve this modification, the background electron density is multiplied by a factor $w$, dependent on the distance $r_{\mathrm{H}, \mathrm{Au}}$ between $\mathrm{H}$ and $\mathrm{Au}$ atom (Fig. 3.51):

$$
w(r)=\frac{2}{3} \frac{1}{1+\exp \left[15.0\left(r_{\mathrm{H}, \mathrm{Au}}-1.0\right)\right]}+1
$$

This modification results in no change between in the total ELD (see overlap of black and

Table 3.31.: Outcomes (\%) of scattering for scattering events resulting from $\mathrm{H}$ atom collision with a $\mathrm{Au}(111)$ surface for nonadiabatic simulations for modifications to the background electron density. The "Surface"-column refers to trajectories wherein $\mathrm{H}$ atoms scattered from $1^{\text {st }}$ layer of the surface. The Roman numerals refer to the lowest subsurface to which penetration occurred. The incidence conditions are $E_{\text {inc }}=3.33 \mathrm{eV}, \theta_{\mathrm{inc}}=45^{\circ}$ along the [101] surface direction, $300 \mathrm{~K}$ and $6 \times 6 \times 6$ cell, number of simulated trajectories: $10^{6}$.

\begin{tabular}{lllllllll}
\hline \hline & \multicolumn{3}{c}{ bounce events } & \multicolumn{4}{c}{ penetrating bounces } \\
Modification & single & double & multi & surface & I & II & III & $>$ III \\
\hline None & 23 & 34 & 43 & 82 & 17 & 1 & 0 & 0 \\
$(1)$ & 22 & 32 & 46 & 81 & 18 & 1 & 0 & 0 \\
$(2)$ & 25 & 35 & 40 & 83 & 16 & 1 & 0 & 0 \\
$(3)$ & 23 & 33 & 43 & 81 & 17 & 1 & 0 & 0 \\
$(4)$ & 17 & 25 & 59 & 64 & 23 & 8 & 3 & 2 \\
\hline \hline
\end{tabular}


Table 3.32.: Energy loss in \% of incidence energy for various outcomes resulting from $\mathrm{H}$ atom collision with a $\mathrm{Au}(111)$ surface for nonadiabatic for modifications to the background electron density. The mean and maximum energy loss are shown for total and differential ELD at $300 \mathrm{~K}$ for $10^{6}$ trajectories. Due to the low signal-to-noise level, the values at specular scattering $\left(\theta_{\text {out }}=45^{\circ} \phi_{\text {out }}=60^{\circ}([10 \overline{1}])\right)$ are shown with less significant figures.

\begin{tabular}{lllll}
\hline \hline & Total & & \multicolumn{2}{c}{$\theta_{\text {out }}=45^{\circ} \phi_{\text {out }}=60^{\circ}$} \\
Modification & Mean & Peak & Mean & Peak \\
\hline none & 35.3 & 14.0 & 37 & 14 \\
$(1)$ & 32.3 & 13.1 & 38 & 13 \\
$(2)$ & 37.1 & 18.2 & 34 & 10 \\
$(3)$ & 35.3 & 15.8 & 37 & 14 \\
$(4)$ & 74.8 & 14.0 & 68 & 13 \\
\hline \hline
\end{tabular}

navy curves in Fig. 3.49(a)), nor in the differential energy loss distribution at specular scattering (Fig. 3.49(b)) and very little in the angular distribution for scattering in [101]-direction (Fig. 3.49(c)). This means that regions of high electron density, where a friction coefficient could be expected to be of larger impact, are either not sampled during a trajectory or play a very insignificant roll for energy losses.

Posteriori (4) electronic friction post-facto as described by Kroes and coworkers [3, 4] where the energy loss to electron hole pair is estimated by Eq. 3.4. The velocities, positions and electron densities are taken from the adiabatic MD simulations. The total energy loss distribution

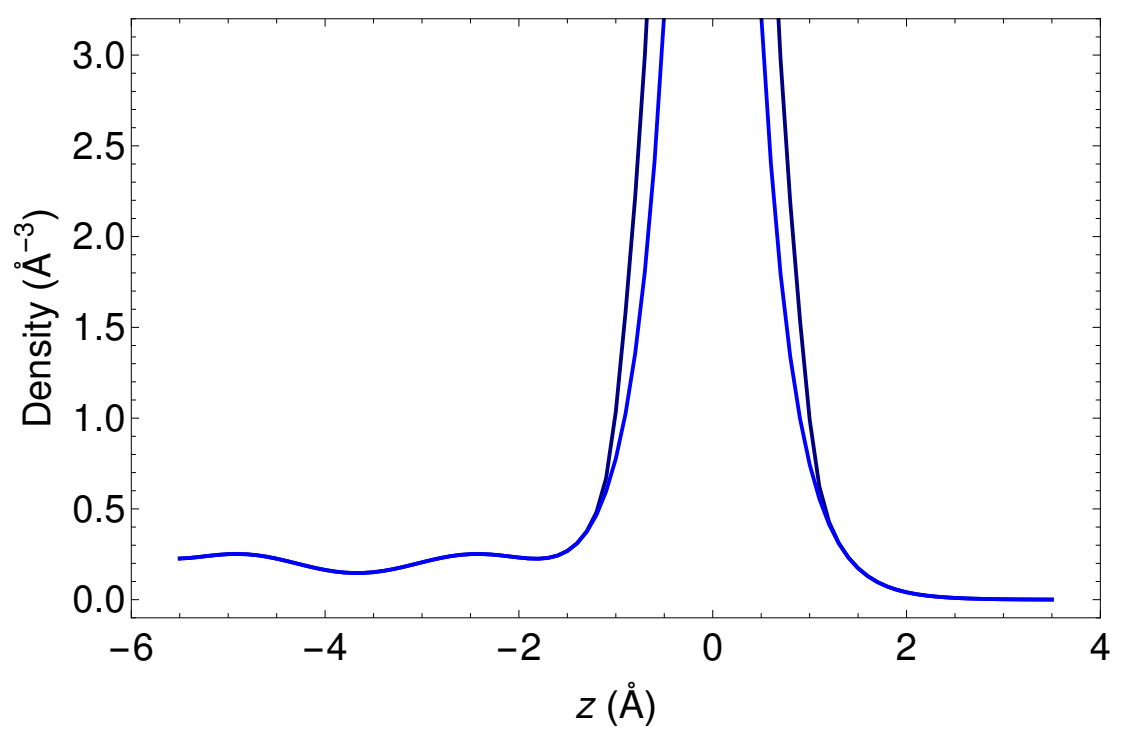

Figure 3.51.: The EMT background electron density of an $\mathrm{H}$ atom (blue) and the modified one defined by Eq. (3.6) (navy) for the top-site. 
(Fig, 3.49(a), yellow) exhibits some similarities with total ELDs at lowered temperature (e.g. $40 \mathrm{~K}$, see section 3.3.2, apart from the fact that the tail of the distribution stretches to very high energy loss values (above $10 \mathrm{eV}$ ) which, given an incidence energy of $3.33 \mathrm{eV}$ appears rather unphysical. The structure of the ELD has little to do with the structure of the ELD from direct nonadiabatic simulations at $300 \mathrm{~K}$, but both peak in the same region of energy loss. Kroes and coworkers [3] found that the first peak is due to non-penetrative collisions and that the nonpenetrative collision exhibit an eight times higher average energy loss in the nonadiabatic case compared to the adiabatic case. This is much larger than the factor of three difference in mean energy loss I find for the comparison of the total energy loss distribution for the nonadiabatic and adiabatic case. Accordingly, Kroes and coworkers estimate from comparison with AIMDEF simulations that the energy transfer to ehp predicted by AIMDEFp should overestimate the energy transfer from direction inclusion of energy loss to ehp by $\sim 20 \%$. Here, I find that the post-facto approach overestimates the mean energy loss to ehp by a factor of 1.8. From both the form of the total ELD and the differential energy loss distribution at specular scattering, it is clear that the post facto approach cannot serve to replace the on-the-fly simulation of energy loss due to electron hole pair excitation.

In summary, I found that large modification of background electron densities have no dramatic influence on the simulation results. These are interesting findings for they suggest that the approach taken by Blanco-Rey et al. [19] during their AIMD simulations including electronic friction to calculate the friction coefficient from the electron densities of an undistorted slab is indeed a reasonable approximation. Furthermore, if only a general impression of the energy loss to electron hole pairs is needed, the calculation of a friction coefficient from the local electron density is not necessary; a well-adjusted constant friction coefficient will also give reasonable ideas.

Most of all, however, that such grave changes in the electron density only affect the outcome of the trajectory to a minor extend, makes it seem unlikely that treating the friction coefficient as a tensor would lead to much different results.

\subsubsection{Conclusion}

In conclusion, I investigated different influences on the energy loss distribution of $\mathrm{H}$ scattering from $\mathrm{Au}(111)$ with and without the inclusion of energy loss to electron hole pair excitation. The difference between the adiabatic and nonadiabatic energy loss distributions is pronounced: while the adiabatic energy loss distributions are very narrow, peak at very low energy loss $(<3 \%$ of incidence energy) and have a low mean energy loss ( $<10 \%$ of incidence energy), the energy loss distributions of nonadiabatic simulations are broad and peak at high energy losses ( $>35 \%$ of incidence energy) while exhibiting a large mean energy loss ( $>30 \%$ of incidence energy). This makes clear that, if energy loss to electron hole pair is included into the MD simulations, the 
energy loss is dominated by it. This agrees well with the predictions for $\mathrm{H}$ at $\mathrm{Au}(111)$ of Kroes and coworkers [3, 4] who estimated the average energy loss to ehp using AIMDEFp and predict that ehp excitation should be the dominant energy dissipation channel and a much broader energy loss distribution for the nonadiabatic case. It is also in accordance with the results the Alducin group [19, 160] obtained with AIMDEF that the energy dissipation to ehp has a large contribution in the relaxation of hot $\mathrm{H}$ on metal surfaces and is about five times faster than the energy dissipation to phonons.

Furthermore, I found the ad- and absorption probabilities of $\mathrm{H}$ at $\mathrm{Au}(111)$, the dynamics of scattering and the dynamics by which the ad/absorption sites were reached to be fundamentally different in the adiabatic and nonadiabatic case. I investigated the energy loss behavior of the nonadiabatic simulations in detail:

Under most incidence conditions, scattering happens predominantly in forward direction. The energy loss distributions exhibit a peak at low energy losses mostly due to single-bounce events, and a shoulder and tail made up by double- and multibounce events. I investigated the dependence of the translational energy loss on different incidence conditions and scattering condition. I found that under the same incidence conditions, for forward scattering, the mean energy loss of the differential ELDs drops slightly towards higher polar scattering and incidence angles. The peak of the differential ELD for forward scattering remains at the same energy losses. For backwards and sidewards scattering, the peak and rising edge shift to higher energy losses and the peak diminishes in favor of the shoulder with increasing $\theta_{\text {out }}$. There is no significant change to be observed in the form of the differential ELD for variation of azimuth and polar incidence angle. The angular distribution in incidence direction is found to be broader toward forward scattering

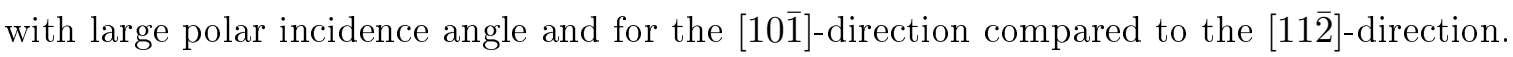

Lowering the simulation temperature leads to a separation of peaks due to different bounce events and at $\sim 40 \mathrm{~K}$, some of the peaks can be attributed to distinct bounce events. Changes in the incidence energy show alike behavior of the energy loss distributions between $E_{\text {inc }}=0.99$ and $3.33 \mathrm{eV}$ such as almost linear dependence of the peak position and mean energy loss on the incidence energy. For $E_{\text {inc }}>5.0 \mathrm{eV}$ the energy loss distributions change as a new peak arises.

Changes in both surface structure and the background electron density have little influence on the energy loss behavior.

The energy loss distribution resulting from the scattering of deuterium from $\mathrm{Au}(111)$ shows only little differences to the one from $\mathrm{H}$ scattering, because the isotope effect on the energy loss to phonons and ehp operates into opposite directions in the simulations. 


\subsection{Surface Reconstruction}

I have simulated the primary reconstruction pattern of the $\mathrm{Au}(111)$ surface consisting of discommensuration lines using EMT-JAWK. For this, I focused on the $22 \times \sqrt{3}$ reconstruction, as this has been the focus of previous DFT simulations [41, 43]. To test for the temperature dependent stability of the discommensuration lines, I used a number of $22 \times 6 \times l$ slabs (with $l=4-15$ and $l=21$ ) with a $13-\AA$ vacuum distance. In the surface layer, one additional atom per atom line in the [110] direction was added, resulting in a $23 \times 6$ surface cell. Using the structure suggested by Wang et al. 41] as an initial guess, I let the slab undergo simulated annealing of ten cycles for $l<13$, five for $l=13$ to 15 and four for $l=21$, keeping the lowest three layers fixed to their relaxed lattice positions. The simulated annealing was performed using the extended Verlet algorithm as a thermostat, each cycle starting at $0 \mathrm{~K}$ and progressing in ten temperature intervals to $T_{\max }$ before progressing to $0 \mathrm{~K}$ in another ten intervals. Each step was equilibrated for 5 ps so that one annealing cycle progressed over $100 \mathrm{ps}$ using a propagation step of $1 \mathrm{fs}$.

Using $T_{\max }=700$ and $800 \mathrm{~K}$, I checked the surface structure after the annealing was completed For $T_{\max }=700 \mathrm{~K}$, the discommensuration lines were stable for all sampled number of layers $l$ whereas for $T_{\max }=800 \mathrm{~K}$ the discommensuration lines were found to decompose into an unreconstructed surface with an island of six atoms on the surface for $l=13$; for $l=4,8$, and 15 the discommensuration lines partially decomposed with three adatoms on the surface. After another annealing cycle for $l=8$, the surface reconstruction had also completely decomposed, forming an unreconstructed surface with an island on the surface. For $l=4$ and $l=15$, the partial decomposition remained stable for another four annealing cycles (400 ps) and the discommensuration lines could not be obtained again. I therefore conclude that the $22 \times \sqrt{3}$ surface reconstruction is stable up to at least $T_{\max }=700 \mathrm{~K}$ and becomes unstable around $T_{\max }=800 \mathrm{~K}$. These findings are in reasonable agreement with the x-ray diffraction studies of Huang et al. [182] where they observed that, at $\sim 865 \mathrm{~K}$, the reconstruction begins to lose its long-range order and forms a more isotropic phase.

Fig. 3.52 (a) shows the surface corrugation after reconstruction for different numbers of layers, $l$, in the form of a height profile. With $l>9$, the difference in height is almost negligible while for fewer layers, the discommensuration lines are still influenced by the fact that slab has only a limited number of layers. The maximum corrugation height $h_{\max }-h_{0}=0.12 \AA$ for $l=6$ and $0.2 \AA$ for $l=20$. These values, as well as slight differences between the depth of the minima, are in good agreement with the corrugation heights found experimentally by Wöll et al. [236] of $0.15 \pm 0.04 \AA$ and by Barth et al. [32] of $0.12 \pm 0.05 \AA$ for the narrower regions and $0.20 \pm 0.05 \AA$ for the wider regions. The structural features of the reconstruction were further studied using the structure of a four-layered slab. Fig. 3.52(b) shows that the atoms of the reconstruction have the lowest height when they lie directly over the $f c c$-hollow sites (green) and the second lowest 

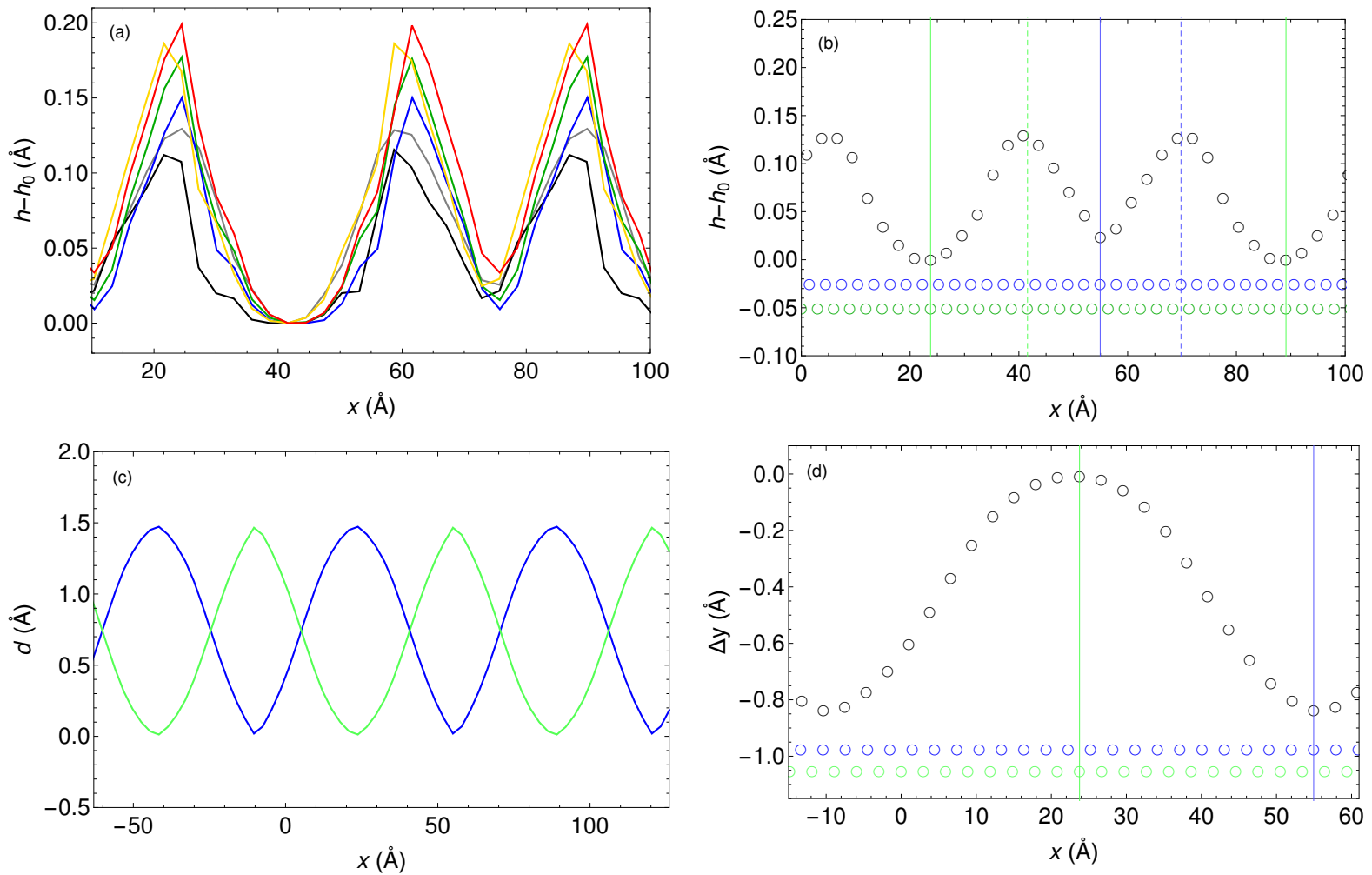

Figure 3.52.: Height profile of the $22 \times \sqrt{3}$ discommensuration line along the [11̄0]-direction (a) for 4 (gray), 6 (black), 9 (blue), 12 (green), 15 (yellow) and 21 (red) layers, (b) for surface atoms (black) in relation to the atoms of the first (blue) and second (green) sublayer. (c) Distance $d$ in $x$-direction to the closest $f c c$ (green) and $h c p$ (blue) site. (d) displacement in $y$-direction of the discommensuration line (black) with respect to ideal $f c c$ lattice $y$ coordinate and the atoms of the first (blue) and second (green) sublayer. The individual lines have been shifted in $x$-direction by multiples of the next-neighbor distance. They have furthermore been multiplied according to periodic boundary conditions.

when they are directly above the $h c p$-hollow (blue) sites. Likewise, when the atoms of the first layer are half covering the hollow sites (so over the bridge sites), the largest heights are reached. This is in excellent agreement with DFT simulations [41-43], which predict that the highest surface atoms correspond to the greatest mismatch of the surface atoms with the atoms of the layers below in the bridge regions and that the deepest minimum is that above the $f c c$-sites. Hanke et al. [43] furthermore classified the atoms of the discommensuration lines according to whether they were closer to the $f c c$ or $h c p$-hollow sites, finding $f c c$-regions with a width of $38 \AA$, and $h c p$-regions with a width of $28 \AA$, in agreement with the experimental observation that the $f c c$-regions are wider than the $h c p$-regions [32, 43]. In very good agreement with this, I find the $f c c$-regions (Fig. 3.52 (c), green) to be $36 \AA$ wide and the $h c p$-regions with a width of $30 \AA$. In $y$-direction, the discommensuration line is bent by $\sim 0.8 \AA$ in [11 $\overline{2}]$-direction and reaches its maximum in displacement above an $h c p$-site (Fig. 3.52(d)), as also described by Torres et 
al. [42]. The displacement of the discommensuration line of $\sim 0.8 \AA$ in [11 $\overline{2}]$-direction is also in agreement with the $\sim 0.9 \AA$ displacement observed experimentally [32].

Furthermore, using a six-layered slab, I calculated the energy $\Delta E$ required to form the $22 \times \sqrt{3}$ $(23 \times \sqrt{3})$ reconstructed surface according to the way by which Hanke [43], Wang [41] and Torres et al. 42] proceeded in their DFT simulations:

$$
\Delta E=E_{\text {rec }}-E_{\text {unrec }}-n E_{\text {Au,bulk }}
$$

Here, $E_{\text {rec }}$ is the total potential energy of a reconstructed slab, $E_{\text {unrec }}$ is the total potential energy of an unreconstructed slab, $n$ is the number of extra atoms in the reconstructed surface and $E_{\mathrm{Au} \text {,bulk }}$ is the bulk energy of an Au atom. The bulk energy is chosen because an Au atom inside the bulk is deemed the most stable Au atom available, making $\Delta E$ thus a lower limit of the actual surface stability. In EMT, $E_{\mathrm{Au}, \text { bulk }}=0.0 \mathrm{eV}$. Using Eq. (3.7), I find the reconstructed surface to be less stable than the unreconstructed surface by $0.31 \mathrm{eV}(0.25 \mathrm{eV})$ for a $22 \times 1 \times 6$ $(23 \times 1 \times 6)$ slab. This corresponds to $13 \mathrm{meV}(10 \mathrm{meV})$ per surface atom that would be required to build up a reconstructed surface. These results are in disagreement with the DFT simulations of Wang [41], Hanke [43] and Torres [42] who observed that the reconstruction is energetically favorable with between -9 and $-25 \mathrm{meV}$ per surface atom. Because the surface reconstruction hosts one extra atom per discommensuration line compared to the unreconstructed surface, I also examined if having an adatom on the surface is more or less favorable than the reconstructed surface and found that the reconstructed surface is $\Delta E=-0.57 \mathrm{eV}(-0.63 \mathrm{eV})$ more stable than the unreconstructed surface with an adatom for a $22 \times 1 \times 6(23 \times 1 \times 6)$ slab. This corresponds to $-25 \mathrm{meV}(-26 \mathrm{meV})$ per surface atom. It means that, while the formation of the surface reconstruction might be unfavorable compared to the perfect structure in EMT-JAWK, it is much more favorable than creating adatoms on the surface. Accordingly, the reconstructed surface does not expell the additional atom immediately during simulated annealing, but only does so at higher temperatures when the thermal motion becomes strong enough to push it out.

To see if, due to the large lattice constant of $a_{0}=4.201 \AA$ of the EMT-JAWK, another reconstruction pattern is more stable, I checked the dependence of the stability of the surface reconstruction on the size of the reconstruction. For this, I used an underlying $b \times 6 \times 6$ slab where $b$ is the slab size index and was sampled for $b=22-44$, with one extra atom per discommensuration line. I performed simulated annealing to a maximum temperature of $700 \mathrm{~K}$ with ten annealing cycles, again keeping the three lowest layer fixed. Fig.3.53 shows that, for all slab sizes, the surface reconstruction is less stable than the unreconstructed surface. The closer the surface comes to the unreconstructed structure, the more energetically favorable the system. However, the inset shows that at the same time, adatoms on the surface are much less stable than the reconstructed surface. 


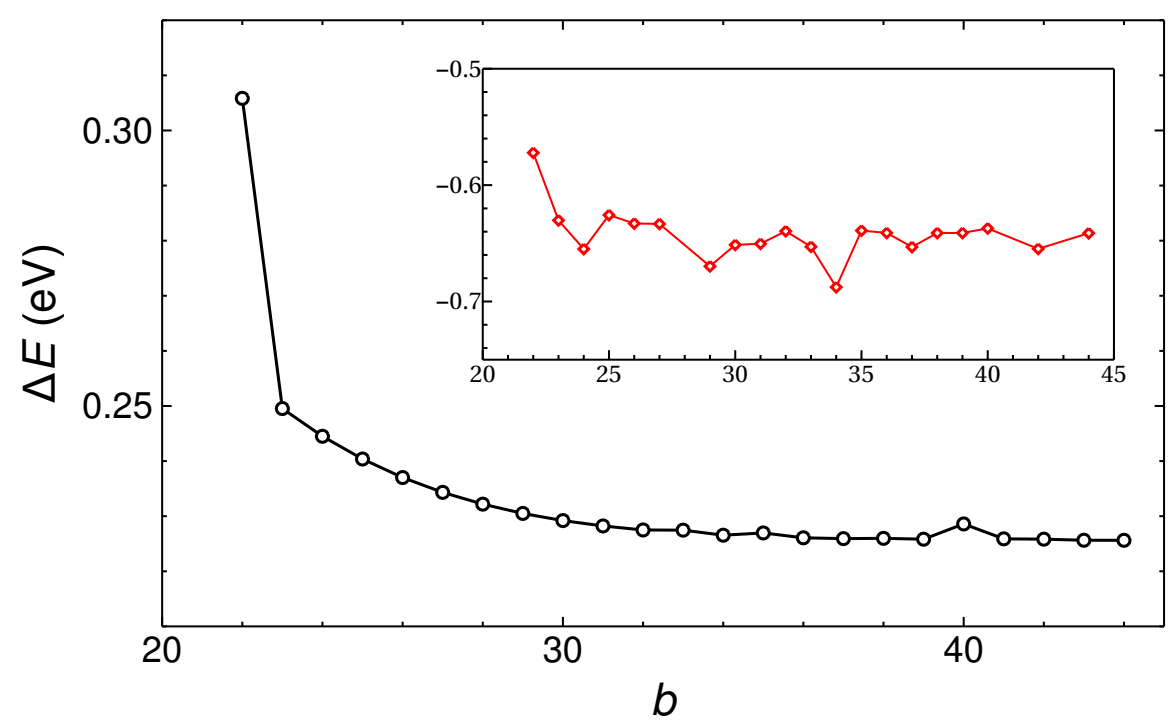

Figure 3.53.: Energy of reconstruction $\Delta E$ as a function of the slab size index $b$ for the difference between reconstructed and unreconstructed surface (black) and, in the inset, for reconstructed surface and unreconstructed surface with adatom.

In modeling the surface reconstruction, the EMT-JAWK is capable of reproducing the most important geometrical features that have been described using DFT. However, the reconstruction in EMT-JAWK is not as energetically favorable as the one found with DFT. But it should be remembered that Eq. (3.7) provides an upper limit to estimate the stability of the surface reconstruction. Simulated annealing over at least $400 \mathrm{ps}$ has shown that, up to a temperature of at least $700 \mathrm{~K}$, the reconstruction remains perfectly stable. Furthermore, the approach taken with Eq. (3.7) appears a little ad hoc as it is unlikely that a Au atom would move up from the bulk to form the surface reconstruction. It seems much more likely that the surface reconstruction is formed to avoid forming small islands on the surface or having adatoms. Indeed, if the energy difference between the reconstructed surface and an unreconstructed surface with adatoms is calculated, the reconstruction proves much more favorable.

Seeing that the discommensuration lines are stable to annealing up to high temperatures within the EMT-JAWK approach opens the way to further studies dealing with the $\mathrm{Au}(111)$ surface reconstruction: EMT-JAWK allows the simulation of much larger structures than possible with DFT. In the future, the secondary reconstruction, the herringbone pattern, could be investigated as well as the reconstruction at step edges and the elbow- and U-shaped patterns the reconstruction assumes. 


\section{Comparison of MD simulations to Experimental Results}

In this chapter I compare the scattering results simulated with EMT-JAWK reported in the previous sections with results of inelastic scattering of $\mathrm{H}$ atoms from a $\mathrm{Au}(111)$ surface which Oliver Bünermann, Yvonne Dorenkamp and Hongyan Jiang measured using the experimental setup described in our paper [44]. They were able to create a nearly monoenergetic $\mathrm{H}$ atom beam by laser photolysis of hydrogen iodide. Dependent on the photons used for dissociation and the spin-orbit state (either ${ }^{2} P_{1 / 2}$ or ${ }^{2} P_{3 / 2}$ ) of HI whose dissociation products are selected for photolysis, the incidence energy of the $\mathrm{H}$ atoms can be varied. After scattering, the $\mathrm{H}$ atoms are detected by Rydberg atom tagging with a detector which can be rotated to detect atoms scattered in the plane of incidence as a function of the final polar angle $\theta_{\text {out }}$. They detect $\mathrm{H}$ atom time-of-flight (TOF) which are transformed with a Jacobian to translational energy distributions [44].

In this chapter, I will concentrate on the comparison of theoretical predictions for energy loss distributions and the experimental results. I will also present a brief comparison of predicted and measured angular distributions. Some of the comparisons made here have already been published (see Ref. [44]). For the sake of clarity, within this chapter, the 'differential energy loss distributions' have been abbreviated to 'energy loss distribution'. Within this chapter, the term 'energy loss distribution' will therefore always refer to the differential energy loss distributions.

\subsection{Energy Loss Distributions}

All the conditions probed experimentally are listed in Tab.4.1. In Figs.4.1, 4.2, 4.3 and 4.4 I show comparisons of energy loss distributions between experiment and MD simulation for different exit conditions, different incidence conditions, the dependence on the energy loss and the isotope effect, respectively. Conditions listed in Tab4.1 for which no comparison are shown exhibit similar behavior.

The energy loss distributions from electronically adiabatic MD simulation all show a most probable energy loss of $<0.1 \mathrm{eV}$ (Figs. 4.1 and 4.2 , blue). The energy loss distributions are sharply peaked, narrow, with little contribution to the energy loss above $1.0 \mathrm{eV}$. The measured 
Table 4.1.: Incidence conditions probed experimentally. The surface was kept at a temperature of $300 \mathrm{~K}$.

\begin{tabular}{llll}
\hline$E_{\text {inc }}(\mathrm{eV})$ & $\theta_{\text {in }}\left(^{\circ}\right)$ & $\phi_{\text {in,out }}\left(^{\circ}\right)$ & $\theta_{\text {out }}\left({ }^{\circ}\right)$ \\
\hline Hydrogen & & & \\
\hline 3.33 & 20 & {$[10 \overline{1}]$} & 20,60 \\
& 30 & {$[10 \overline{1}]$} & 30,60 \\
& 45 & {$[10 \overline{1}]$} & $0,15,30,45,60$ \\
& 60 & {$[10 \overline{1}]$} & 0,60 \\
& 45 & {$[11 \overline{2}]$} & 45 \\
2.76 & 45 & {$[10 \overline{1}]$} & 45 \\
2.62 & 45 & {$[10 \overline{1}]$} & 0,45 \\
2.17 & 45 & {$[10 \overline{1}]$} & 0,45 \\
1.92 & 45 & {$[10 \overline{1}]$} & 0,45 \\
1.22 & 45 & {$[10 \overline{1}]$} & 45 \\
0.99 & 45 & {$[10 \overline{1}]$} & 0,45 \\
\hline Deuterium & & & \\
\hline 3.27 & 45 & {$[10 \overline{1}]$} & 45 \\
3.27 & 45 & {$[11 \overline{2}]$} & 45 \\
\hline \hline
\end{tabular}

energy loss distributions (black squares) peak around $0.5 \mathrm{eV}$ energy loss and are very broad having a very wide tail to high energy losses up to the incidence energy $(3.33 \mathrm{eV})$. The mean energy loss has a linear dependence on the incidence energy (Fig 4.3 (c)) for both measured data (black squares) and electronically adiabatic MD simulations (blue, filled circles), but both mean energy loss and slope differ considerably. From the discrepancy in shape, tail, peak position and dependence of the mean energy loss on the incidence energy, it is clear that the adiabatic level of simulation is not suited to describe the experimental results. It should however be pointed out that the adiabatic simulations predict a decline of energy loss with growing polar incidence angle for forward scattering, a trend that is also observed experimentally.

If energy loss to electron hole pairs on the level of LDFA is included into the MD simulation and the resulting energy loss distribution (Figs. 4.1 and 4.2 red) compared to experiment, the results are very different: the nonadiabatic energy loss distributions peak at $E_{\text {loss }} \sim 0.5 \mathrm{eV}$, are very broad in shape and have a long tail that reaches up to complete loss of all translational incidence energy. They are in good agreement with the experimental data, not only for the general shape but also for peak and tail description of the translational energy loss distribution. This agreement holds for all exit conditions (Fig.4.1) and incidence conditions, no matter the incidence angle (Fig. 4.2) or the incidence energy (Fig.4.3). The decline of polar incidence angle 

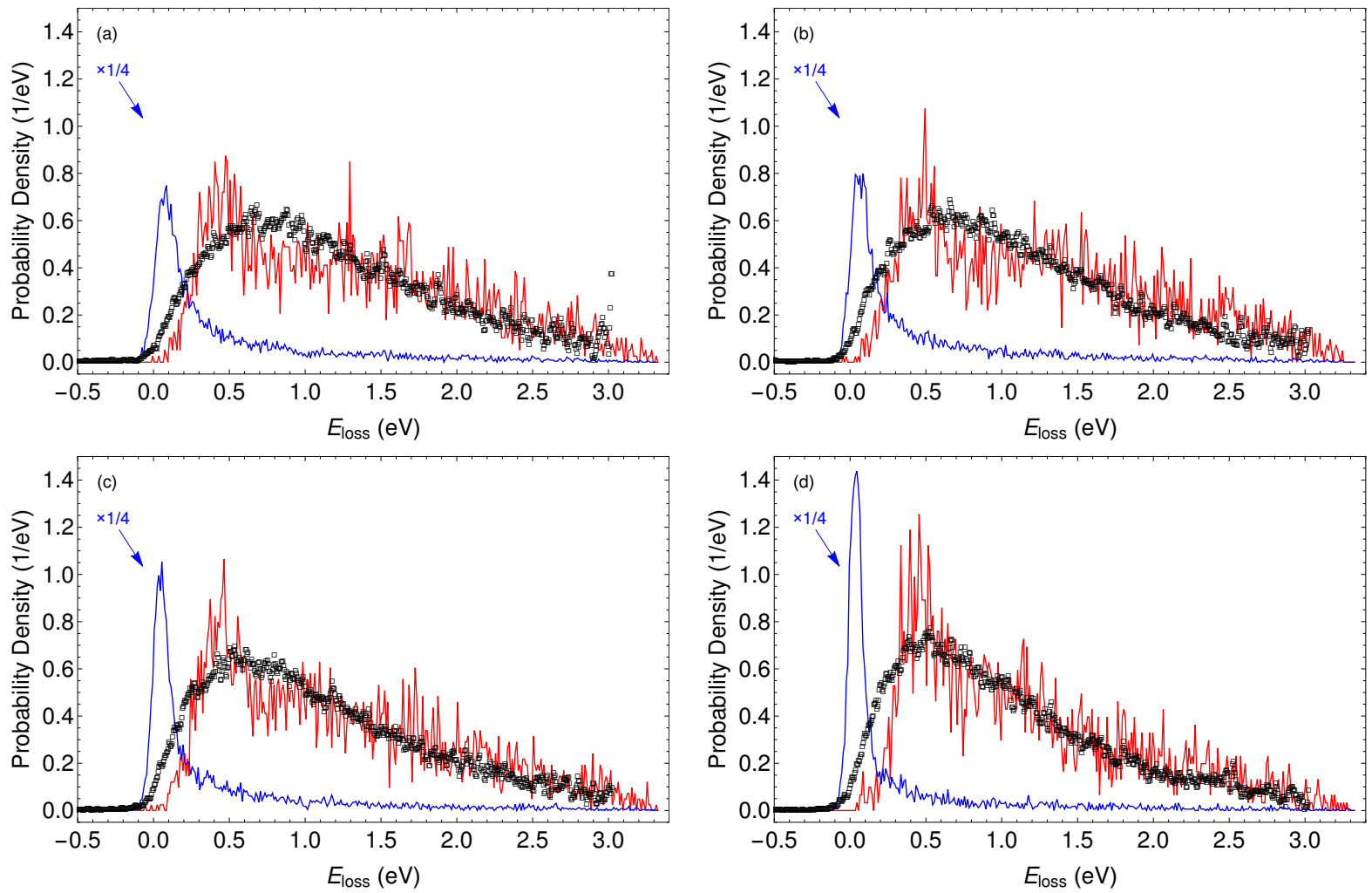

Figure 4.1.: Energy loss distribution of experiment (black squares) and the electronically adiabatic (blue) and nonadiabatic (red) distributions from MD simulations for different exit conditions. Incidence conditions: $E_{\text {inc }}=3.33 \mathrm{eV}, \theta_{\text {in }}=45^{\circ}$ along the [101] -direction (a) $\theta_{\text {out }}=0^{\circ}$ (b) $\theta_{\text {out }}=15^{\circ}$ (c) $\theta_{\text {out }}=30^{\circ}$ (d) $\theta_{\text {out }}=60^{\circ}$.

(polar scattering angle) at fixed scattering angle (fixed incidence angle) leads to a slight decline in mean energy loss observed both for the measured and nonadiabatically simulated ELDs. The dependence of the predicted mean energy loss on the incidence energy (Fig.4.3. (c) red, open circles) is in very good agreement with the measurements (black, squares).

The agreement between ELD measured experimentally and from nonadiabatic simulations also holds for deuterium (Fig.4.4).

From these comparisons of experiment and theory, it is clear that $\mathrm{H}$ or $\mathrm{D}$ atoms colliding with $\mathrm{Au}(111)$ lose most of their translational energy to electron hole pair excitation and that the ehp excitation can be described on the level of LDFA. This does not mean that energy loss to phonons can be completely neglected in the simulations; as already shown in section 3.3 .2 , the reduction of phonon activity at reduced temperature leads to a strong separation of energy peaks due to single-, double- and multibounce scattering. The phonons have an important "blurring effect", however, it is clear from the minor contribution of phonon excitation to the peak positions (also those of the different bounce events) that the translational energy is dominantly dissipated to ehp excitation. 

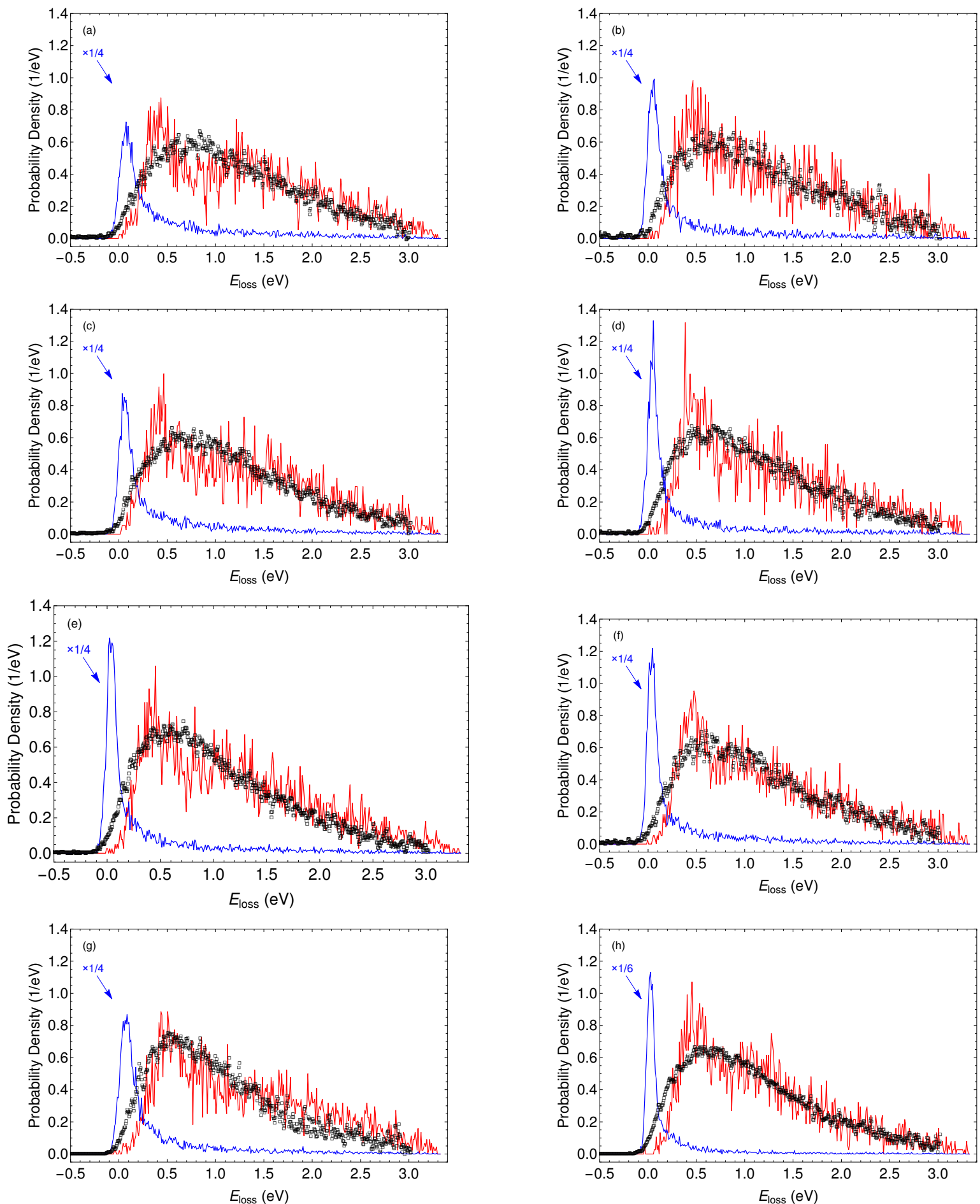

Figure 4.2.: Dependence of Energy loss distribution of experiment (black squares), electronically adiabatic (blue) and nonadiabatic (red) MD simulations at $E_{\text {inc }}=3.33 \mathrm{eV}$ on different inci-

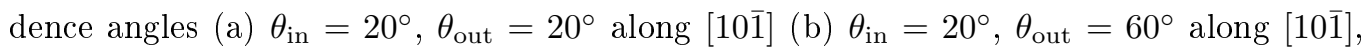
(c) $\theta_{\text {in }}=30^{\circ}, \theta_{\text {out }}=30^{\circ}$ along [101] (d) $\theta_{\text {in }}=30^{\circ}, \theta_{\text {out }}=60^{\circ}$ along [101] (e) $\theta_{\text {in }}=45^{\circ}$, $\theta_{\text {out }}=45^{\circ}$ along $[10 \overline{1}],(7) \theta_{\text {in }}=45^{\circ}, \theta_{\text {out }}=45^{\circ}$ along $[11 \overline{2}]$ (h) $\theta_{\text {in }}=60^{\circ}, \theta_{\text {out }}=0^{\circ}$ along $[10 \overline{1}](\mathrm{j}) \theta_{\text {in }}=60^{\circ}, \theta_{\text {out }}=60^{\circ}$ along $[11 \overline{2}]$. 

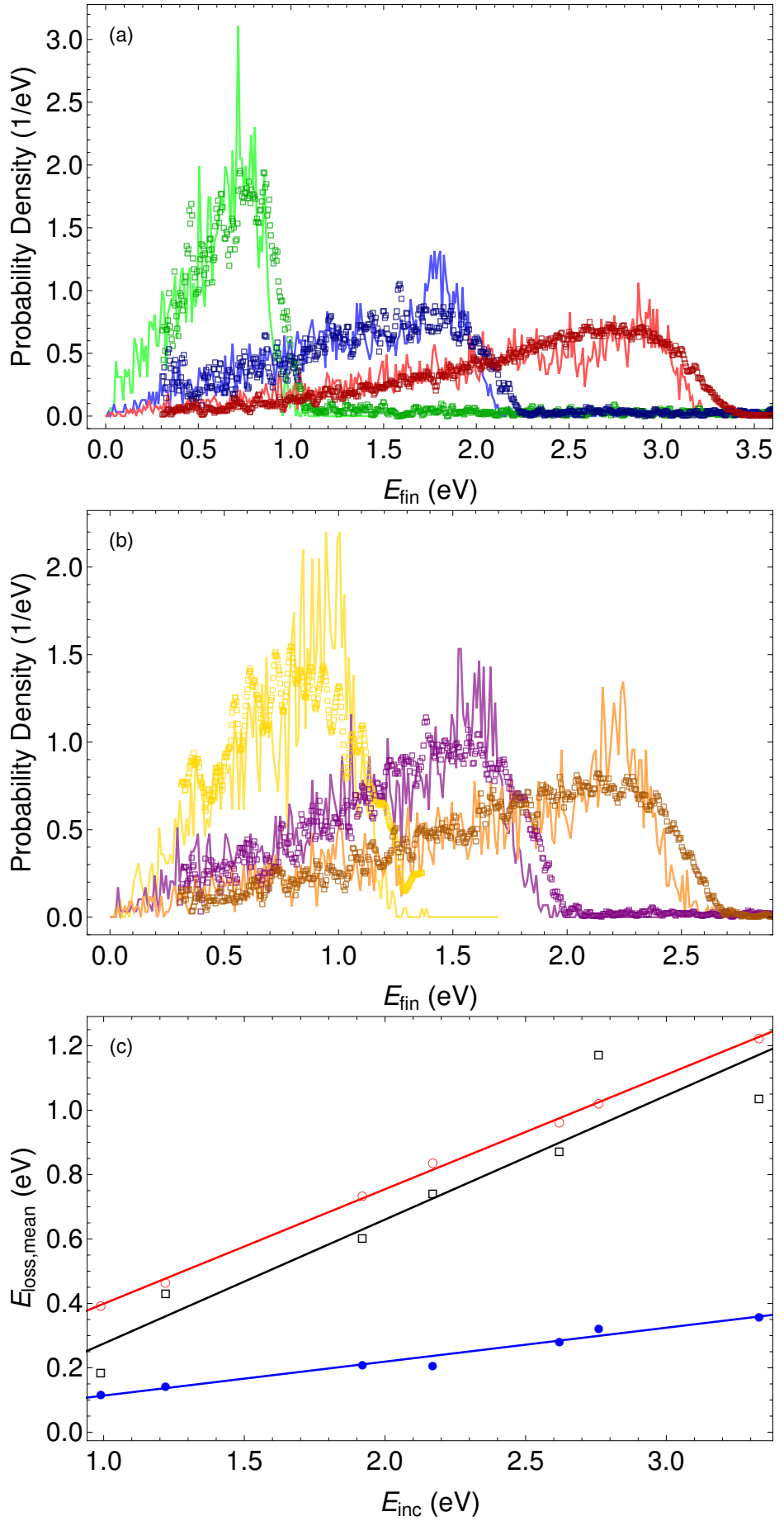

Figure 4.3.: Experimentally measured translational energy distribution (squares) and that from nonadiabatic MD-simulations (solid lines) for specular scattering for $\theta=45^{\circ}$ and $\phi=60^{\circ}$ (a) For $E_{\text {inc }}=3.33 \mathrm{eV}$ (red), $E_{\text {inc }}=2.17 \mathrm{eV}$ (blue), $E_{\text {inc }}=0.99 \mathrm{eV}$ (green) (b), $E_{\text {inc }}=2.62 \mathrm{eV}$ (orange), $E_{\mathrm{inc}}=1.92 \mathrm{eV}$ (purple), $E_{\mathrm{inc}}=1.22 \mathrm{eV}$ (yellow). (c) Mean energy loss of energy loss distributions plotted against incidence energy from experimental measurements (black, empty squares) and electronically adiabatic (blue, filled circles) and nonadiabatic (red, empty circles) MD-simulations. The solid lines indicate a linear least square fit to the data. These results have been partly published [44]. 
As good as the agreement between the nonadiabatic MD simulations and the measured translational energy loss distribution is, there are still some systematic deviations. The peak of the measured energy loss distribution descends continuously into the tail at high energy losses. The energy loss distribution from the nonadiabatic MD-simulations shows a sharp peak followed by a dip and a shoulder before it slopes off into a long tail at high energy losses. The shape of the ELD at the shoulder changes slightly which leads to slightly better agreement at high polar scattering and incidence angles. Furthermore, the calculations underestimate the intensity of the energy loss at low energy losses and the peak is shifted to slightly lower energy losses than the peak of the measured ELDs at low $\theta_{\text {in/out }}$. As described in the previous chapter 3.3 , the peak in the ELD from the nonadiabatic MD simulations is mostly due to energy losses resulting from single-bounce collision with the surface while the shoulder is caused in large part by double-bounce collisions overlapping at low energy losses with the ELD of the single-bounce events and at high energy losses with the energy loss distribution due to multibounce collisions. Presumably, the MD-simulations either overestimate the percentage of single-bounce events or underestimate the percentage of double-bounce events (see Fig.3.16(b)). Possibly, given that MD-simulations do not entirely reproduce the low energy loss region, the energy loss distribution of the single-bounce events might also be represented as being too narrow, underestimating the contribution of high and low energy loss single-bounce events.

For the experimental ELDs, it was found that the fractional energy loss $\Delta \bar{E}_{\text {loss }} / E_{\text {inc }}=0.33 \pm$ 0.01 is almost independent of the incidence energy [44]. I performed a linear least square fit using the linear least square procedure provided by the Mathematica package [237] of the mean energy loss as a function of the incidence energies $E_{\text {inc }}=3.33 \mathrm{eV}, 2.76 \mathrm{eV}, 1.92 \mathrm{eV}, 0.99 \mathrm{eV}, 2.62 \mathrm{eV}$, $2.17 \mathrm{eV}$ and $1.22 \mathrm{eV}$. The contribution of the last three incidence energies is not included in the publication in Ref. [44]. I obtain a fractional energy loss of 0.39 with an rms-error of 0.11 for the experimental ELDs, $0.35 \pm 0.01$ and $0.11 \pm 0.01$ for the ELDs from the nonadiabatic and adiabatic MD-simulations (see also Fig.4.2(c)). The results from the nonadiabatic simulations are, as expected, in very good agreement with those from the experimental measurements.

From Fig. 3.37(a) it can be seen that, for the region sampled by the experimental measurements, the change in the ratio between mean energy loss and incidence energy is very small. It would therefore be very interesting to see what happens at higher incidence energies. For one, to see if the relation between mean energy loss and incidence energy behaves as predicted by the calculations and secondly, to see if, with higher incidence energies, the energy loss distribution changes as much as the calculations predict (see Sec.3.3.3).

The agreement between the nonadiabatic simulations for $\mathrm{D}$ scattering off $\mathrm{Au}(111)$ and the measured energy loss are slightly better than those for $\mathrm{H}$ scattering off $\mathrm{Au}(111)$ (see Fig.4.4. It is clear that the MD-simulations including low-energy ehp excitation offers an explanation for the apparent lack of isotope effect observed experimentally (see section 3.3.6): the doubled 

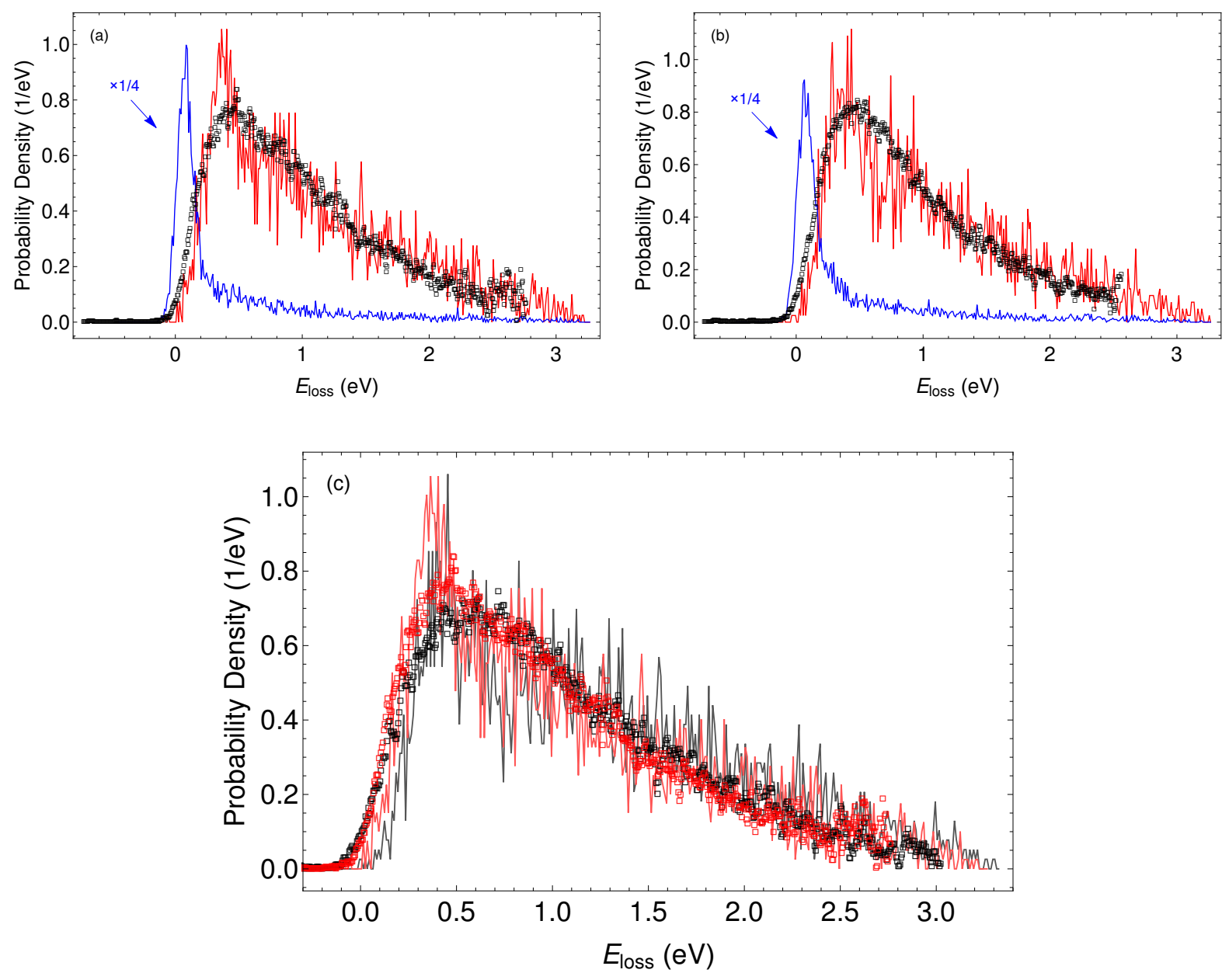

Figure 4.4.: Experimentally measured energy loss distribution (black squares), electronically adiabatic (blue) and nonadiabatic (red) MD-simulations for deuterium scattering from $\mathrm{Au}(111)$ (a)

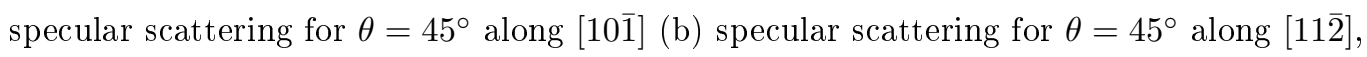
(c) Energy loss distributions measured experimentally (squares) and from nonadiabatic MD simulations (solid lines) for deuterium (red) and hydrogen (black).

energy loss of $\mathrm{D}$ to phonons compared to $\mathrm{H}$ is compensated by a lower energy loss of $\mathrm{D}$ compared to $\mathrm{H}$ to ehps.

\subsection{Angular Distributions}

Fig. 4.5 shows the angular distribution along [101] for adiabatic (blue) and nonadiabtic (red) simulations in comparison to the measured angular distribution. As remarked in section. 3.3.1, there is little difference between the adiabatic and nonadiabatic distribution. The agreement of both of them with the measured angular distribution is good. The angular distribution measured experimentally peaks very close to the surface normal at low $\theta_{\text {in }}$ and moving to larger ones at larger $\theta_{\text {in }}$. The peak from the simulated energy loss distributions is always almost at the same 
$\theta_{\text {out }}$. Furthermore, the calculations capture the general shape of the experimentally measured energy loss distribution, even if they overestimate its wideness. Close to normal incidence, the angular distribution appears narrower and with larger polar incidence angle slants more and more towards larger polar scattering angles. As can be seen from the constant difference at high scattering angles between the experimental and simulated angular distribution, the amount of slanting is very well reproduced by the simulations. Additionally, with growing polar incidence angle, the angular distribution appears to grow wider, a trend that is also captured by the simulations. The good agreement with experimental measurements shows that, while interactions with ehp does not appear to influence the angular distribution greatly, the interaction with phonons does. A comparison to the angular distributions for low temperatures (see Fig. 3.31 makes evident that a solid surface approach would be hard pressed to describe the experimental findings.
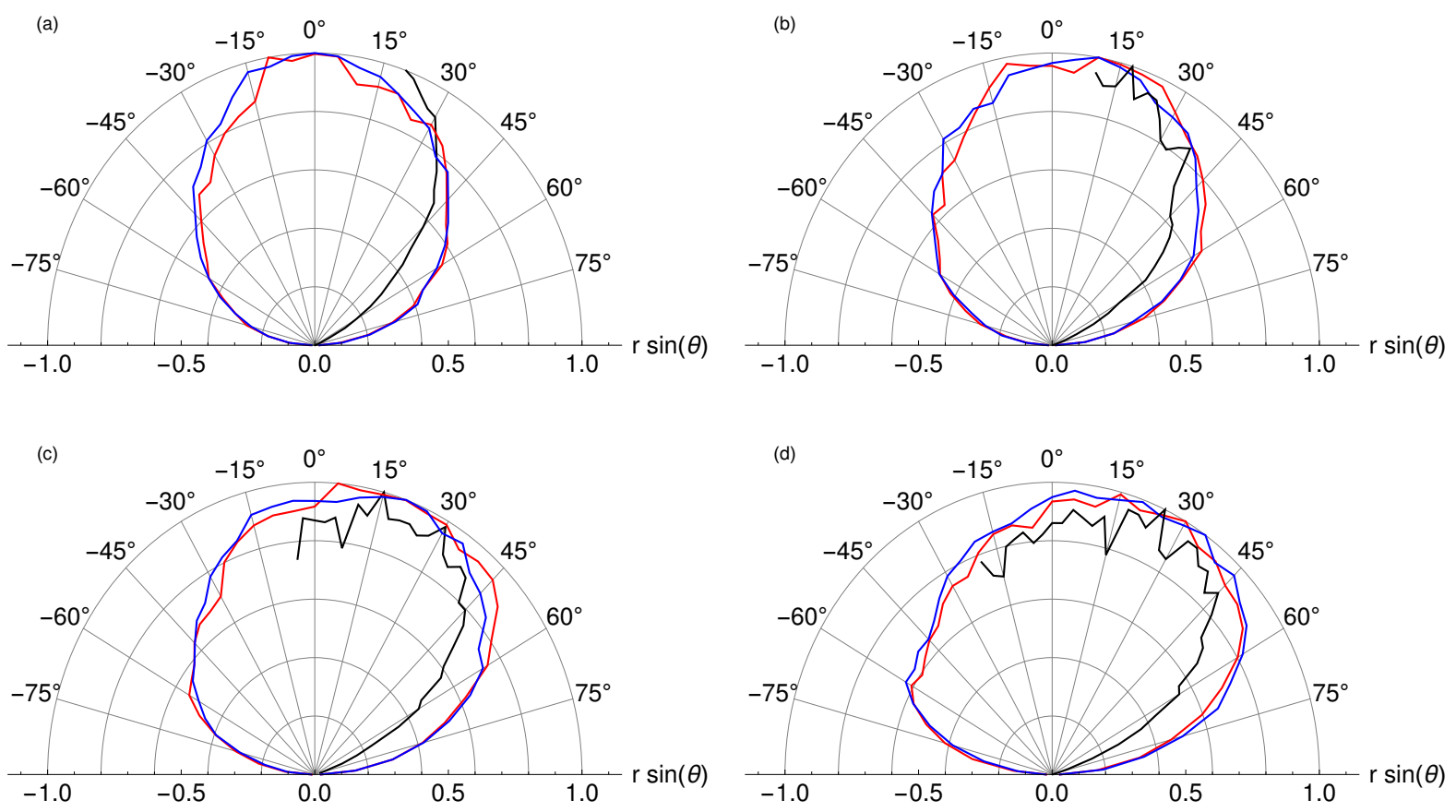

Figure 4.5.: Angular distribution along the $[10 \overline{1}]$-direction of scattering $\mathrm{H}$ atoms for experiment (black) for the electronically adiabatic (blue) and nonadiabatic (red) case. Different polar incidence angles along the [101] -direction are shown (a) $\theta_{\text {in }}=20^{\circ}$, (b) $\theta_{\text {in }}=30^{\circ}$, (c) $\theta_{\text {in }}=45^{\circ}$ (d) $\theta_{\text {in }}=60^{\circ}$. Distributions are normalized to maximum and negative polar angle corresponds to backscattering. 


\subsection{Improvement of comparison}

Although the agreement between simulation and experiment is reasonably good, the results from the nonadiabatic MD-simulations do differ from those of the experiment. This could be owing to a number of reasons. As described in section 4.1, the number of trajectories experiencing low energy losses is underestimated in the calculations in comparison to experiment while the peak of the energy loss distribution is overestimated. This could be due to an underestimate of singlebounce trajectories with very high and low energy losses, that is, the energy loss distribution of the single-bounce events might be too sharply represented. Furthermore, it seems plausible that low energy double-bounce scattering events are likewise underestimated. Possible causes for such discrepancies could be as follows: (1) That the surface structure is falsely represented. During the simulations above, surface reconstruction has been neglected. Using a slab in the calculations that includes surfaces reconstruction comes at cost of considerable increase in computational effort: increasing from a $6 \times 6$ cell to the $22 \times 6$ cell would increase the number of atoms per layer by a factor of nearly four, not to mention the six additional atoms in the first layer due to the surface reconstruction. Even if four layers instead of six are used, the calculation time still more than doubles. In section 3.3.5, I have already pointed out that the influence of surface reconstruction (or even adatoms on the surface) does not appear to have a great effect on the energy loss distributions. In Fig.4.6(b), the ELD from nonadiabatic simulations at specular scattering angles is shown in comparison to the ELD measured experimentally. The inclusion of surface reconstruction improves the representation of the shoulder, the peak is still slightly overestimated and the low energy loss events still underestimated. The slight improvement in shape scarcely merits the large computational effort.

(2), From the observation that the energy loss distributions of the bounce events are too narrow and that, at higher surface temperature, the energy loss distribution of the different bounce events become broader, the corrugation of the surface at $300 \mathrm{~K}$ could be larger than simulated with EMT-JAWK. Increasing the surface temperature to counterbalance a possibly too low surface corrugation at a simulated temperature leads to a better capturing of the low energy loss contributions, but the strength of the peak of the energy loss distribution and its tail are still overestimated while the shoulder is still underestimated (Fig.4.6(c)).

(3) The background electron density extracted from the EMT could be faulty. In Sec.3.3.7, several different modifications to the density were considered and from the results of this section, it is evident that the deviation between theory and experiment is not solely due to a misrepresentation of the background electron density. Lowering the background electron density to fit that of the $a b$ initio calculations would lead to a better representation of the low energy losses, but then fail to capture the position of the peak and would not address the issue of the too weak shoulder. Applying constant density (Fig.4.6.(d), navy) improves the treatment of the 

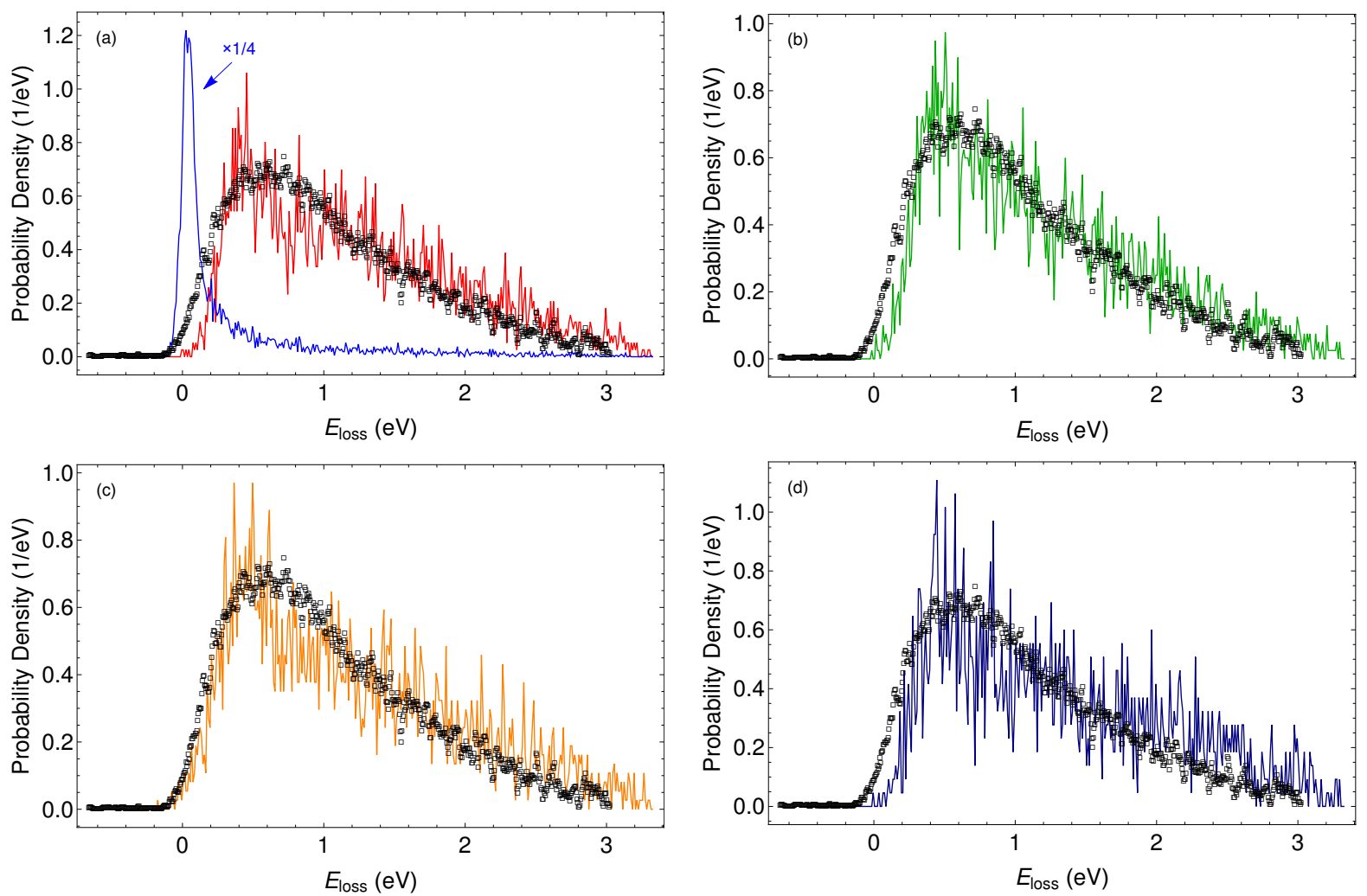

Figure 4.6.: Energy loss distribution (a) from the experiment (black squares) in comparison with the electronically nonadiabatic (red) and adiabatic (blue); (b) experiment (black squares) and nonadiabatic simulations with reconstructed surface (green); (c) experiment (black squares) and nonadiabatic simulations with relaxed surface at $500 \mathrm{~K}$ (orange) for specular scattering at $E_{\text {inc }}=3.33 \mathrm{eV}$ and $\theta_{\text {in }}=45^{\circ}, \phi_{\text {in }}=60^{\circ}$ at $300 \mathrm{~K}$. (d) nonadiabatic simulations (blue) with constant density inside the surface.

shoulder somewhat, but leads to a stronger overestimation of the distribution tail and a worse representation of the low energy loss region.

(4) Further reasons for the disagreement could be that EMT-JAWK does not include effects such as dispersion or that the theoretical treatment of electron hole pair excitation in the frame of local density friction approximation is insufficient or that quantum effects play a role. These are more serious. To address the first issue, the EMT functional form would have to changed. With regards to the treatment of electron hole pair excitation, it appears that the general assumption made by the use of LDFA, that only energetically low lying electron hole pairs are excited, is valid. Staying in the frame of LDFA, the theory can still be improved. At the moment, we are using a scalar for the position dependent friction acting upon the hydrogen atom instead of a friction matrix. Although section 3.3.7 has shown that slight modifications in the density do not lead to great changes, including a friction tensor would mean that an $\mathrm{H}$ atom would not experience the same background electron density in all directions and might thereby be 
influenced in the path it takes through or along the Au-slab. These effects can be expected to be small for scattering atoms, perhaps influencing the dynamics of sticking more than those of scattering. Nonetheless, the inclusion of a friction tensor appears to be worthwhile, if only just as a prove of principle.

Lastly, $\mathrm{H}$ being a rather small particle, quantum effects could affect its scattering and its inclusion is under way.

\section{Estimation of zero-point energy effects}

Following up on the idea that the surface corrugation might be underestimated, I want to estimate how large this effect could be and if it would have any influence on the temperature predicted for the separation of peaks due to different bounce events in sec.3.3.2 at low temperatures. From a quantum-mechanical point of view for a surface at $0 \mathrm{~K}$ the square mean deviation $\left\langle x^{2}\right\rangle$ of the surface atoms from their relaxed lattice positions is not zero. For a quantum-mechanical harmonic oscillator, the mean square displacement can be expressed as [175]

$$
\left\langle x^{2}\right\rangle=\left(v+\frac{1}{2}\right) \frac{\hbar}{m \omega} .
$$

Here, $m$ is the mass and $\omega$ is the frequency of the oscillation and $v$ is the quantum number of the vibrational state. The mean potential energy of the oscillator in the ground state is:

$$
\left\langle V_{0}\right\rangle=\frac{\hbar \omega}{4}
$$

In the following, I will study this effect qualitatively to get an estimate of how much zero-point motion might influence the displacement of the atoms at different temperatures. I calculate the relation between $\left\langle x^{2}\right\rangle$ and the temperature for a bath of harmonic oscillators, and. For this, I replace the 'real' system described by the EMT by a reference system of classical harmonic oscillators. Their mean potential energy can be calculated as

$$
\langle V\rangle=\frac{1}{2} m \omega^{2}\left\langle x^{2}\right\rangle .
$$

According to the equipartition theorem of classical mechanics, the average energy per degree of freedom for a thermal bath of harmonic oscillators at the temperature $T$ is

$$
\langle V\rangle=\frac{k_{\mathrm{B}} T}{2}
$$

Substituting Eq. (4.3) into Eq. (4.4) yields the classical frequency $\omega$ so that it now can be calculated from the mean square displacement at different temperatures.

$$
\omega=\sqrt{\frac{k_{\mathrm{B}} T}{m\left\langle x^{2}\right\rangle}}
$$


If the real system indeed behaved like harmonic oscillators, then $\omega$ should be constant with varying temperature. For an estimate of the mean square displacement, I take 1000 configurations of $216 \mathrm{Au}$ atom positions simulated over $10 \mathrm{ps}$. Tab.4.2 shows the mean square displacement I obtain in this manner for several temperatures. I can now presume this frequency to correspond

Table 4.2.: Estimate of zero-point motion.

\begin{tabular}{llll}
\hline \hline$T(\mathrm{~K})$ & $\left\langle x^{2}\right\rangle(\AA)$ & $\omega\left(10^{12} \cdot \mathrm{s}^{-1}\right)$ & $T_{\text {shift }}(\mathrm{K})$ \\
\hline 40 & 0.075 & 5.05 & 19.3 \\
120 & 0.114 & 5.73 & 21.9 \\
300 & 0.173 & 5.98 & 22.8 \\
400 & 0.189 & 6.34 & 24.2 \\
500 & 0.212 & 6.32 & 24.1 \\
600 & 0.225 & 6.50 & 24.8 \\
700 & 0.240 & 6.60 & 25.2 \\
800 & 0.256 & 6.61 & 25.2 \\
\hline \hline
\end{tabular}

to the frequency of the quantum-mechanic oscillator and calculate its $\left\langle x^{2}\right\rangle$ in the ground state from Eq. 4.3). Substituting this value into Eq. 4.4), I can find the temperature $T_{\text {shift }}$ which I would have to simulate for a bath of harmonic oscillators (or the real system at the given temperature $T$ ) to reproduce the zero-point motion. Looking at Tab. 4.2 this means that to account for zero-point motion effects at $40 \mathrm{~K}$, I would have to perform classical simulations at $60 \mathrm{~K}$. Or, rather, the predictions made in section 3.3 .2 for $40 \mathrm{~K}$ would predictions for $20 \mathrm{~K}$ in experiment.

\subsection{Conclusion}

From the above considerations it is undoubtedly clear that the adiabatic picture is incapable of describing the energy loss observed experimentally for an $\mathrm{H}$ atom scattering from $\mathrm{Au}(111)$ : neither peak position nor overall form agree to any extend with the experimentally measured differential ELD. In comparison, if energy dissipation to ehp is included by means of LDFA, the agreement between experiment and theory is very good for all sampled conditions, including different incidence and scattering angles, different incidence energies and a full recovery of the isotope effect. The modeling of the isotope effect and the angular distribution along [101] direction shows that, although energy loss to ehp is by far the dominating energy pathway, the inclusion of energy loss to phonons must not be neglected.

If the angular distribution along [10̄1]-direction is considered, the discrepancy between nonadiabatic and adiabatic distribution is very small, but also in good agreement with the experimental observations. 
The agreement between nonadiabatic simulations and measured distributions is not perfect. A search for causes unearthed that the disagreement is not caused by a neglect of the $22 \times \sqrt{3}$ reconstruction, nor by ignoring the possible effects of zero-point motion or a bad guess of the background electron density. Further investigations need to be made in this direction, especially towards the inclusion of quantum effects or the use of a friction matrix.

The good agreement between nonadiabatic MD-simulations and experimental measurements make it further likely that simulations done for higher and lower incidence energy are adequate predictions and that the sticking mechanisms described in section 3.3.1 carry weight. Small shape changes in shoulder of the differential ELD from the nonadiabatic simulations do not appear to occur to such large extends in the experiment. The lowering of the energy loss with smaller polar incidence and scattering angles that is observed both experimentally and in the MD-simulations is only visible in the MD-simulations for forward scattering, not for side- and backwards scattering. It would therefore be interesting to see if this is also observed experimentally. Furthermore, it remains to be explored if a lowering of the surface temperature in experiment leads to a more distinct splitting of the differential ELD into peaks and if those peaks split so decisively at very low surface temperature that that their origin can be explained by the present simulations. 



\section{Conclusions and Outlook}

In summary, I constructed a full-dimensional potential energy surface (PES) for $\mathrm{H}$ at $\mathrm{Au}(111)$ that reproduces $a b$ initio DFT calculations with good accuracy and an rms-error of $\sim 160 \mathrm{meV}$. To obtain the PES, I fitted the analytic form of the effective medium theory (EMT) to DFT energies. To perform the fit, and following MD-simulations, I used the MD_tian-package, a FORTRAN code which I wrote and contributed to substantially. Constraints for the fit were derived from the bulk properties of $\mathrm{Au}$ and the experimental binding energies of the $\mathrm{H}-\mathrm{Au}$ molecule. Depending on how the fit was done, different sets of parameters were obtained, however, a comparison of different PESs resulting from these parameter sets shows that although some parameters might differ to a large extend, once constrains for the fit are chosen properly, all parameter sets give rise to PESs that produce the same behavior in MD simulations. I denoted the PES [22] that I used for the simulations presented here with EMT-JAWK.

A comparison to scattering simulations using ab initio molecular dynamics (AIMD) [3, 4] shows that the adiabatic total energy loss distribution, scattering and event probabilities obtained with AIMD and EMT-JAWK are in very good agreement with one another. Given the good agreement, the use of the potential energy surface appears preferable to AIMD due to the large computational effort AIMD requires: the EMT-JAWK allows me to perform simulations over a wide range of incidence conditions using large simulation cells and long interaction times at reasonable computational effort.

Furthermore, the EMT analytic expressions supply background electron densities which allows to implement electronic friction self-consistently and thereby to obtain a method to describe the interaction with electron hole pairs. Changes in the background density are found to be of little effect on the energy loss behavior.

I find that the inclusion of nonadiabatic effect prodigiously increases the energy loss of scattering $\mathrm{H}$ atoms by a factor of three in mean energy loss and more than a factor of ten in the position of the peak of the total energy loss distribution. This is in agreement with predictions about the importance of nonadiabatic effects made for the energy loss behavior to electron hole pairs using AIMD with the LDFA applied in a post-facto approach [3, 4] and the predominant energy loss to ehp observed for $\mathrm{H}$ at $\mathrm{Pd}$ using the AIMD with the inclusion of friction on-thefly [19]. Different from the AIMD approaches, the EMT based approach presented here uses the background electron densities that is needed for LDFA instead of the electron densities of only 
the bare surface atoms. The background electron density and that of the bare surface atoms differ but modification of the electron densities for different MD simulations shows that changes in the electron densities influence the outcome of the simulations only marginally.

MD simulations on the EMT-JAWK PES allow full-dimensional treatment involving hundreds of atoms on a picosecond timescale with millions of trajectories. This ability allows to calculate a sufficiently large number of trajectories to make predictions for both adsorption probabilities and angle resolved measurement of inelastic scattering. It allows me to identify scattering regions where different scattering behavior and at low temperature even mechanisms might be distinguished experimentally. Notably, it also allows me to predict mechanisms by which adsorption takes place. I have predicted the energy loss behavior for multiple temperatures and incidence conditions. A variation in incidence angle as well as detected scattering angle shows only little influence on the position and form of the differential energy loss distribution. The largest variation can be achieved with changes in the surface temperature and incidence energy. For high incidence energies $\left(E_{\text {inc }}>7.0 \mathrm{eV}\right)$, I was able to predict a very much differently shaped energy loss distribution than for low incidence energies.

Simulations of the $22 \times \sqrt{3}$ reconstruction of the $\mathrm{Au}(111)$ surface show that the reconstruction is stable up to at a temperature of at least $\sim 700 \mathrm{~K}$, energetically favorable compared to adatoms on the surface, but not to the unreconstructed surface as found in DFT simulations [41-43], and in good agreement with experimental observations [32].

From comparison to experimental results it becomes obvious that the experimentally observed energy loss is dominated by energy dissipation into electron hole pair excitation. The inclusion of energy dissipation to phonons is necessary in the description of isotope effect and angular distributions. Comparison of nonadiabatic simulations to experimental results shows very good agreement with the overall energy loss behavior for different incidence energies, incidence angles and scattering directions: the theory developed here is capable of describing the overall energy loss behavior observed experimentally and moreover shows that single $\mathrm{H}$ atoms in interaction with metal surface lose most of their energy to excitation of low lying electron hole pairs. The lack of an isotope observed experimentally is reproduced by the theory and could be rationalized by a combination of adiabatic and nonadiabatic effects.

Slight deviations between experiment and theory persist. An investigation of the influence of changes in surface structure or electron density shows little or no improvement. Further investigations of the discrepancy should be the inclusion of semi-classical or quantum mechanical effects for the treatment of the $\mathrm{H}$ atom (an estimate of the influence of zero-point energy has shown little influence) as well as attempts to treat the friction as a tensor instead of a scalar as done here.

Further experimental investigation that appear to be promising according to the predictions made here would be a lowering of surface temperature for which I predict the resolution of 
different peaks in the energy loss distribution that can be attributed to different scattering mechanisms such as single- or double-bounce scattering. Also, an increase in the experimental incidence energy would provide a good test to see if the theory is also capable of predicting this behavior correctly: for $E_{\text {inc }}>7.0$, the form of the energy loss distribution changes considerably, including larger nonadiabatic vs adiabatic effects at $E_{\mathrm{inc}}=10.0 \mathrm{eV}$. Measurements of sticking probability would provide another very sensitive test to the theory.

There are interesting future direction for the theory. One involves the energetic behavior in the DFT-calculations for the octahedral sites. During the construction of the PES, I found that this behavior is different for $\mathrm{Au}$ than for other metals. Here, work is required to determine the origin of this interesting behavior. A second area of interest is the determination of the melting temperature. At the moment, it has to be obtained from MD-simulations performed at different temperatures. It would certainly be advantageous if the melting temperature of the surface could be related to parameter values of the PES. A large field of interest is also the investigation of the surface reconstruction. The PES (or one fitted specifically for Au with no $\mathrm{H}$ atom present) could be used to investigate the properties of the secondary surface reconstruction pattern of $\mathrm{Au}(111)$, the herringbone, especially in view of the large simulation boxes that are possible with the PES.

There are many more interesting future direction, including the effect of a matrix friction coefficient, the inclusion of semi-classical effects, describing molecules at surfaces by combining this approach with Lennard-Jones or similar potentials. It would also be interesting to extend the theory to more than two-component systems so that scattering from alloys or covered surfaces could be investigated. Yet another interesting topic is that of diffusion. Li and Wahnström [30] showed that nonadiabatic effects have little influence on the self-diffusion coefficient; it will be most interesting to see if we observe similar behavior.

Finally, an obvious and much needed future direction is the extension of the theory to different metal surfaces. This work is already under way. 



\section{Bibliography}

[1] W. Bligh, ADM 55/151, The National Archives, Admiralty papers, Public Record Office, Kew, Richmond, Surrey, UK, 1787-1789.

[2] K. W. Jacobsen, P. Stoltze, J. K. Nørskov, Surface Science 1996, 366, 394-402.

[3] G.-J. Kroes, M. Pavanello, M. Blanco-Rey, M. Alducin, D. J. Auerbach, Journal of Chemical Physics 2014, 141, 054705.

[4] M. Pavanello, D. J. Auerbach, A. M. Wodtke, M. Blanco-Rey, M. Alducin, G.-J. Kroes, Journal of Physical Chemistry Letters 2013, 4, 3735-3740.

[5] C. Díaz, R. A. Olsen, H. F. Busnengo, G. J. Kroes, Journal of Physical Chemistry C 2010, $114,11192-11201$.

[6] C. Diaz, R. A. Olsen, D. J. Auerbach, G. J. Kroes, Phys. Chem. Chem. Phys. 2010, 12, 6499-6519.

[7] J. C. Polanyi, Accounts of Chemical Research 1972, 5, 161.

[8] D. Townsend, S. A. Lahankar, S. K. Lee, S. D. Chambreau, A. G. Suits, X. Zhang, J. Rheinecker, L. B. Harding, J. M. Bowman, Science 2004, 306, 1158-1161.

[9] M. H. Qiu, Z. F. Ren, L. Che, D. X. Dai, S. A. Harich, X. Y. Wang, X. M. Yang, C. X. Xu, D. Q. Xie, M. Gustafsson, R. T. Skodje, Z. G. Sun, D. H. Zhang, Science 2006, 311, 1440-1443.

[10] S. Nave, B. Jackson, Phys. Rev. Lett. 2007, 98, 173003.

[11] M. Bonfanti, C. Díaz, M. F. Somers, G.-J. Kroes, Physical Chemistry Chemical Physics 2011, 13, 4552-4561.

[12] F. Nattino, C. Diaz, B. Jackson, G. J. Kroes, Physical Review Letters 2012, 108, 236104.

[13] J. D. White, J. Chen, D. Matsiev, D. J. Auerbach, A. M. Wodtke, Nature 2005, 433, $503-505$.

[14] Y. Huang, C. T. Rettner, D. J. Auerbach, A. M. Wodtke, Science 2000, 290, 111-4. 
[15] N. Shenvi, S. Roy, J. C. Tully, Science 2009, 326, 829-832.

[16] M. Head-Gordon, J. C. Tully, Journal of Chemical Physics 1995, 103, 10137-10145.

[17] R. Baer, Y. Zeiri, R. Kosloff, Phys. Rev. B 1997, 55, 10952-10952.

[18] J. K. Nørskov, B. I. Lundqvist, Surface Science 1979, 89, 251-261.

[19] M. Blanco-Rey, J. I. Juaristi, R. Diez Muino, H. F. Busnengo, G. J. Kroes, M. Alducin, Physical Review Letters 2014, 112, 103203.

[20] P. Saalfrank, J. I. Juaristi, M. Alducin, M. Blanco-Rey, R. D. Muiño, The Journal of Chemical Physics 2014, 141, 234702.

[21] S. M. Janke, M. Pavanello, G.-J. Kroes, D. J. Auerbach, A. M. Wodtke, A. Kandratsenka, Zeitschrift für Physikalische Chemie 2013, 227, 1467-1490.

[22] S. M. Janke, D. J. Auerbach, A. M. Wodtke, A. Kandratsenka, Journal of Chemical Physics 2015, $143,124708$.

[23] J. Strömquist, L. Bengtsson, M. Persson, B. Hammer, Surface Science 1998, 397, 382-394.

[24] I. Langmuir, Journal of the American Chemical Society 1912, 34, 860-877.

[25] M. Andersson, F. Abild-Pedersen, I. Remediakis, T. Bligaard, G. Jones, J. Engbæk, O. Lytken, S. Horch, J. Nielsen, J. Sehested, J. Rostrup-Nielsen, J. Nørskov, I. Chorkendorff, Journal of Catalysis 2008, 255, 6 - 19.

[26] D. Hollenbach, E. E. Salpeter, Phys. Rev. B 1971, 163, 155-164.

[27] S. M. Lee, K. S. Park, Y. C. Choi, Y. S. Park, J. M. Bok, D. J. Bae, K. S. Nahm, Y. G. Choi, S. C. Yu, N. Kim, T. Frauenheim, Y. H. Lee, Synthetic Metals 2000, 113, 209 216.

[28] M. E. Tuckerman, Journal of Physics-Condensed Matter 2002, 14, R1297-R1355.

[29] R. Car, M. Parrinello, Physical Review Letters 1985, 55, 2471-2474.

[30] Y. Li, G. Wahnström, Physical Review B 1992, 46, 14528-14542.

[31] J. Juaristi, M. Alducin, R. Díez Muiño, H. F. Busnengo, A. Salin, Physical Review Letters 2008, 100, 116102.

[32] J. V. Barth, H. Brune, G. Ertl, R. J. Behm, Physical Review B 1990, 42, 9307-9318.

[33] M. V. Hove, R. Koestner, P. Stair, J. Bibérian, L. Kesmodel, I. BartoŠ, G. Somorjai, Surface Science 1981, 103, 189 - 217. 
[34] U. Harten, A. M. Lahee, J. P. Toennies, C. Wöll, Phys. Rev. Lett. 1985, 54, 2619-2622.

[35] E. M. McIntosh, P. R. Kole, M. El-Batanouny, D. M. Chisnall, J. Ellis, W. Allison, Phys. Rev. Lett. 2013, 110, 086103.

[36] J. K. Nørskov, N. D. Lang, Physical Review B 1980, 21, 2131-2136.

[37] J. K. Nørskov, Physical Review B 1982, 26, 2875-2885.

[38] K. W. Jacobsen, J. K. Nørskov, M. J. Puska, Physical Review B 1987, 35, 7423-7442.

[39] J. Greeley, M. Mavrikakis, The Journal of Physical Chemistry B 2005, 109, 3460-3471.

[40] A. Groß, Theoretical Surface Science. A Microscopic Perspective, Springer-Verlag, Berlin, Heidelberg, 2009.

[41] Y. Wang, N. S. Hush, J. R. Reimers, Physical Review B 2007, 75, 233416.

[42] E. Torres, G. A. DiLabio, The Journal of Physical Chemistry C 2014, 118, 15624-15629.

[43] F. Hanke, J. Björk, Phys. Rev. B 2013, 87, 235422.

[44] O. Bünermann, H. Jiang, Y. Dorenkamp, A. Kandratsenka, S. M. Janke, D. J. Auerbach, A. M. Wodtke, Science 2015, 350, 1346-1349.

[45] J. C. Tully in Dynamics of Molecular Collisions, W. H. Miller (Ed.), Plenum, 1976, pp. $217-267$.

[46] D. J. Tannor, Introduction to Quantum Mechanics: A Time-Dependent Perspective, of Oxford science publications, University Science Books, 2007.

[47] D. Marx, J. Hutter in Modern Methods and Algorithms of Quantum Chemistry, Vol. 3, J. Grotendorst (Ed.), John von Neumann Institute for Computing, 2000, pp. 329-477.

[48] A. Nitzan, Chemical Dynamics in Condensed Phases: Relaxation, Transfer, and Reactions in Condensed Molecular Systems, of Oxford science publications, Oxford University Press, 2006.

[49] M. Born, R. Oppenheimer, Annalen der Physik 1927, 389, 457-484.

[50] A. M. Wodtke, J. C. Tully, D. J. Auerbach, International Reviews in Physical Chemistry 2004, 23, 513-539.

[51] D. J. Auerbach, A. M. Wodtke in Dynamics of Gas-Surface Interactions, R. D. M. no, H. F. Busnengo (Eds.), Springer, 2013, pp. 267-297. 
[52] H. S. W. Massey, Reports on Progress in Physics 1949, 12, 248-269.

[53] J. Thompson, Philosophical Magazine 1905, 10, 584-590.

[54] R. Ryberg, Surface Science 1982, 114, 627-641.

[55] H. C. Chang, G. E. Ewing, Physical Review Letters 1990, 65, 2125-2128.

[56] M. Head-Gordon, J. C. Tully, The Journal of Chemical Physics 1992, 96, 3939-3949.

[57] B. Persson, M. Persson, Solid State Communications 1980, 36, 175 - 179.

[58] B. Schindler, D. Diesing, E. Hasselbrink, The Journal of Chemical Physics 2011, 134, 034705 .

[59] B. Mildner, E. Hasselbrink, D. Diesing, Chemical Physics Letters 2006, 432, 133 - 138.

[60] M. P. Allen, D. J. Tildesley, Computer Simulation of Liquids, of Oxford science publications, Oxford University Press, 1989.

[61] M. P. Allen in Computational Soft Matter: From Synthetic Polymers to Proteins, Lecture Notes, Vol. 23, N. Attig, K. Binder, H. Grubmüller, K. Kremer (Eds.), NIC Series, 2004, pp. 1-28.

[62] W. Koch, M. C. Holthausen, A Chemist's Guide to Density Functional Theory 2nd ed., Wiley-VCH Verlag GmbH, 2001.

[63] D. Marx, J. Hutter, Ab Initio Molecular Dynamics, Cambridge University Press, 2009.

[64] J. Behler, International Journal of Quantum Chemistry 2015, 115, 1032-1050.

[65] L. M. Raff, R. Komanduri, M. Hagan, S. T. S. Bukkapatnam, Neural Networks in Chemical Reaction Dynamics, Oxford University Press, 2012.

[66] H. F. Busnengo, A. Salin, W. Dong, Journal of Chemical Physics 2000, 112, 7641-7651.

[67] J. Ischtwan, M. A. Collins, Journal of Chemical Physics 1994, 100, 8080.

[68] M. A. Collins, Theorectical Chemistry Accounts 2002, 108, 313-324.

[69] S. Sato, The Journal of Chemical Physics 1955, 23, 592-593.

[70] J. H. McCreery, G. J. Wolken, Journal of Chemical Physics 1975, 63, 2340-2349.

[71] M. S. Daw, M. I. Baskes, Physical Review B 1984, 29, 6443-6453.

[72] M. S. Daw, M. I. Baskes, Physical Review Letters 1983, 50, 1285-1288. 
[73] G. Evangelakis, N. Papanicolaou, Surface Science 1996, 347, 376 - 386.

[74] Y. Li, G. Wahnström, Phys. Rev. Lett. 1992, 68, 3444-3447.

[75] C. Engdahl, G. Wahnström, Surface Science 1994, 312, 429-- 440.

[76] N. Perron, N. Pineau, E. Arquis, J. Rayez, A. Salin, Surface Science 2005, 599, 160 - 172.

[77] C. Arasa, H. Busnengo, A. Salin, R. Sayós, Surface Science 2008, 602, 975 - 985.

[78] N. Pineau, H. F. Busnengo, J. C. Rayez, A. Salin, The Journal of Chemical Physics 2005, 122, 214705.

[79] B. Jiang, H. Guo, Journal of Chemical Physics 2014, 141, 034109.

[80] S. Lorenz, A. Groß, M. Scheffler, Chemical Physics Letters 2004, 395, 210-215.

[81] T. B. Blank, S. D. Brown, A. W. Calhoun, D. J. Doren, Journal of Chemical Physics 1995, 103, 4129-4137.

[82] N. Artrith, J. Behler, Physical Review B 2012, 85, 045439.

[83] J. Frankcombe, T, M. A. Collins, D. H. Zhang, Journal of Chemical Physics 2012, 137, 144701.

[84] C. Crepos, M. A. Collins, E. Pijper, G. J. Kroes, Chemical Physics Letters 2003, 376, $566-575$.

[85] J. Ludwig, D. G. Vlachos, The Journal of Chemical Physics 2007, 127, 154716.

[86] G. Laurent, F. Martín, H. F. Busnengo, Phys. Chem. Chem. Phys. 2009, 11, 7303-7311.

[87] H. F. Busnengo, C. Crespos, W. Dong, J. C. Rayez, A. Salin, The Journal of Chemical Physics 2002, 116, 9005-9013.

[88] H. Busnengo, W. Dong, A. Salin, Chemical Physics Letters 2000, 320, 328 - 334.

[89] G. Volpilhac, H. Busnengo, W. Dong, A. Salin, Surface Science 2003, 544, 329 - 338.

[90] P. Riviére, H. F. Busnengo, F. Martín, The Journal of Chemical Physics 2004, 121, 751760.

[91] H. F. Busnengo, A. E. Martínez, The Journal of Physical Chemistry C 2008, 112, 55795588 .

[92] M. A. Di Cèsare, H. F. Busnengo, W. Dong, A. Salin, The Journal of Chemical Physics 2003, 118, 11226-11234. 
[93] G. Kresse, Phys. Rev. B 2000, 62, 8295-8305.

[94] M. Luppi, D. A. McCormack, R. A. Olsen, E. J. Baerends, The Journal of Chemical Physics 2005, 123, 164702.

[95] A. Lozano, A. Gross, H. F. Busnengo, Phys. Chem. Chem. Phys. 2009, 11, 5814-5822.

[96] R. A. Olsen, H. F. Busnengo, A. Salin, M. F. Somers, G. J. Kroes, E. J. Baerends, The Journal of Chemical Physics 2002, 116, 3841-3855.

[97] A. Salin, The Journal of Chemical Physics 2006, 124, 104704.

[98] J. K. Vincent, R. A. Olsen, G.-J. Kroes, M. Luppi, E.-J. Baerends, The Journal of Chemical Physics 2005, 122, 044701.

[99] S. Sato, The Journal of Chemical Physics 1955, 23, 2465-2466.

[100] A. Forni, G. F. Tantardini, Surface Science 1996, 352-354, 142 - 147, Proceedings of the 15th European Conference on Surface Science.

[101] P. Gamallo, L. Martin-Gondre, R. Sayós, C. Crespos, P. Larrégaray in Dynamics of GasSurface Interactions, R. D. M. no, H. F. Busnengo (Eds.), Springer, 2013, pp. 25-50.

[102] L. Martin-Gondre, C. Crespos, P. Larregaray, J. Rayez, B. van Ootegem, D. Conte, Chemical Physics Letters 2009, 471, 136 - 142.

[103] B. J. Garrison, D. Srivastava, Annual Reviews of Physical Chemistry 1995, 46, 373-394.

[104] P. Koumoutsakos, Multiscale Modeling and Simulation for Fluid Mechanics at the Nanoscale, Wiley-VCH Verlag GmbH \& Co. KGaA, 2008, pp. 229-290.

[105] A. C. T. van Duin, S. Dasgupta, F. Lorant, W. A. G. III, The Journal of Physical Chemistry A 2001, 105, 9396-9409.

[106] M. J. Buehler, A. C. T. van Duin, W. A. Goddard, Phys. Rev. Lett. 2006, 96, 095505.

[107] X. J. Shen, A. Lozano, W. Dong, H. F. Busnengo, X. H. Yan, Physical Review Letters 2014, 112, 046101.

[108] M. S. Daw, S. M. Foiles, M. I. Baskes, Material Science Reports 1993, 9, 251-310.

[109] T. N. Truong, D. G. Truhlar, B. C. Garrett, The Journal of Physical Chemistry 1989, 93, $8227-8239$.

[110] K. Capelle, Brazilian Journal of Physics 2006, 36, 1318-1343. 
[111] C. J. Cramer, Essentials of Computational Chemistry 2nd ed., John Wiley \& Sons, Ltd, 2004.

[112] W. Kohn, L. J. Sham, Phys. Rev. 1965, 140, A1133-A1138.

[113] D. M. Ceperley, B. J. Alder, Physical Review Letters 1980, 45, 566-569.

[114] J. P. Perdew, K. Schmidt, AIP Conf. Proc. 2001, 577, 1-20.

[115] J. P. Perdew, K. Burke, M. Ernzerhof, Phys. Rev. Lett. 1996, 77, 3865-3868.

[116] J. P. Perdew, J. A. Chevary, S. H. Vosko, K. A. Jackson, M. R. Pederson, D. J. Singh, C. Fiolhais, Phys. Rev. B 1992, 46, 6671-6687.

[117] J. P. Perdew, J. A. Chevary, S. H. Vosko, K. A. Jackson, M. R. Pederson, D. J. Singh, C. Fiolhais, Phys. Rev. B 1993, 48, 4978-4978.

[118] Y. Zhang, W. Yang, Phys. Rev. Lett. 1998, 80, 890-890.

[119] M. Dion, H. Rydberg, E. Schröder, D. C. Langreth, B. I. Lundqvist, Phys. Rev. Lett. 2004, 92, 246401.

[120] Y.-Y. Chuang, M. L. Radhakrishnan, P. L. Fast, C. J. Cramer, D. G. Truhlar, The Journal of Physical Chemistry A 1999, 103, 4893-4909.

[121] C. Díaz, E. Pijper, R. A. Olsen, H. F. Busnengo, D. J. Auerbach, G. J. Kroes, Science 2009, 326, 832-834.

[122] S. A. Varganov, R. M. Olson, M. S. Gordon, G. Mills, H. Metiu, The Journal of Chemical Physics 2004, 120, 5169-5175.

[123] N. Chetty, K. Stokbro, K. W. Jacobsen, J. K. Nórskov, Physical Review B 1992, 46, 3798-3809.

[124] H. Ibach, H. Lüth, Festköperphysik, Springer, 2009.

[125] C. Kittel, Introduction to Solid State Physics 8th ed., Wiley \& Sons, Inc., 2010.

[126] A. M. Molenbroek, J. K. Nørskov, B. S. Clausen, The Journal of Physical Chemistry B 2001, 105, 5450-5458.

[127] K. Sato, T. Yoshiie, Q. Xu, Journal of Nuclear Materials 2007, 367-370 A, 382-385.

[128] N. P. Bailey, J. Schiøtz, K. W. Jacobsen, Physical Review B - Condensed Matter and Materials Physics 2006, 73. 
[129] N. P. Bailey, J. Schiøtz, K. W. Jacobsen, Physical Review B 2004, 69, 144205.

[130] J. W. M. Frenken, P. Stoltze, Physical Review Letters 1999, 82, 3500-3503.

[131] J. Meier, J. Schiøtz, P. Liu, J. K. Nørskov, U. Stimming, Chemical Physics Letters 2004, 390, 440-444.

[132] K. Morgenstern, G. Rosenfeld, G. Comsa, M. R. Sørensen, B. Hammer, E. Lægsgaard, F. Besenbacher, Physical Review B - Condensed Matter and Materials Physics 2001, 63, $454121-454125$.

[133] J. Christiansen, K. Morgenstern, J. Schiøtz, K. W. Jacobsen, K. . Braun, K. . Rieder, E. Lægsgaard, F. Besenbacher, Physical Review Letters 2002, 88, 2061061-2061064.

[134] T. Vegge, K. W. Jacobsen, Journal of Physics Condensed Matter 2002, 14, 2929-2956.

[135] T. Vegge, Materials Science and Engineering A 2001, 309-310, 113-116.

[136] T. Vegge, T. Leffers, O. B. Pedersen, K. W. Jacobsen, Materials Science and Engineering A 2001, 319-321, 119-123.

[137] T. Vegge, T. Rasmussen, T. Leffers, O. B. Pedersen, K. W. Jacobsen, Philosophical Magazine Letters 2001, 81, 137-144.

[138] T. Vegge, T. Rasmussen, T. Leffers, O. B. Pedersen, K. W. Jacobsen, Physical Review Letters 2000, 85, 3866-3869.

[139] T. Rasmussen, K. W. Jacobsen, T. Leffers, O. B. Pedersen, S. G. Srinivasan, H. Jónsson, Physical Review Letters 1997, 79, 3676-3679.

[140] F. Pauly, M. Dreher, J. K. Viljas, M. Häfner, J. C. Cuevas, P. Nielaba, Physical Review B - Condensed Matter and Materials Physics 2006, 74.

[141] M. Brandbyge, M. R. Sørensen, K. W. Jacobsen, Physical Review B - Condensed Matter and Materials Physics 1997, 56, 14956-14959.

[142] J. Schiøtz, F. D. Di Tolla, K. W. Jacobsen, Nature 1998, 391, 561-563.

[143] J. Schiøtz, K. W. Jacobsen, Science 2003, 301, 1357-1359.

[144] N. V. Galanis, I. N. Remediakis, G. Kopidakis, Mechanics of Materials 2013, 67, 79-85.

[145] J. Schiøtz, A. E. Carlsson, Philosophical Magazine A: Physics of Condensed Matter, Structure, Defects and Mechanical Properties 2000, 80, 69-82.

[146] J. Schiøtz, T. Leffers, B. N. Singh, Philosophical Magazine Letters 2001, 81, 301-309. 
[147] J. Schiøtz, T. Leffers, B. N. Singh, Radiation Effects and Defects in Solids 2002, 157, 193-200.

[148] K. Sato, T. Yoshiie, T. Ishizaki, Q. Xu, Physical Review B - Condensed Matter and Materials Physics 2007, 75.

[149] K. Sato, T. Yoshiie, Y. Satoh, Q. Xu, Materials Transactions 2004, 45, 833-838.

[150] J. Schiøtz, Materials Science and Engineering A 2004, 375-377, 975-979.

[151] T. Shimokawa, J. J. Mortensen, J. Schiøtz, K. W. Jacobsen, Physical Review B - Condensed Matter and Materials Physics 2004, 69, 214104-1-214104-10.

[152] J. Engbaek, J. Schiøtz, B. Dahl-Madsen, S. Horch, Physical Review B 2006, 74, 195434.

[153] P. Saalfrank, G. Füchsel, S. Monturet, J. C. Tremblay, T. Klamroth in Dynamics of GasSurface Interactions, R. D. M. no, H. F. Busnengo (Eds.), Springer, 2013, pp. 323-348.

[154] E. G. d'Agliano, P. Kumar, W. Schaich, H. Suhl, Phys. Rev. B 1975, 11, 2122-2143.

[155] P. Echenique, R. Nieminen, R. Ritchie, Solid State Communications 1981, 37, 779 - 781.

[156] M. J. Puska, R. M. Nieminen, Phys. Rev. B 1983, 27, 6121-6128.

[157] M. Persson, B. Hellsing, Phys. Rev. Lett. 1982, 49, 662-665.

[158] B. Hellsing, M. Persson, Physica Scripta 1984, 29, 360.

[159] L. Martin-Gondre, M. Alducin, G. A. Bocan, R. Díez Muiño, J. I. Juaristi, Phys. Rev. Lett. 2012, 108, 096101.

[160] D. Novko, M. Blanco-Rey, J. I. Juaristi, M. Alducin, Phys. Rev. B 2015, 92, 201411.

[161] J. R. Trail, D. M. Bird, M. Persson, S. Holloway, The Journal of Chemical Physics 2003, 119, 4539-4549.

[162] S. P. Rittmeyer, J. Meyer, J. I. Juaristi, K. Reuter, Phys. Rev. Lett. 2015, 115, 046102.

[163] G. Füchsel, T. Klamroth, S. Monturet, P. Saalfrank, Phys. Chem. Chem. Phys. 2011, 13, $8659-8670$.

[164] G. Füchsel, S. Schimka, P. Saalfrank, The Journal of Physical Chemistry A 2013, 117, 8761-8769.

[165] L. Diekhöner, L. Hornekær, H. Mortensen, E. Jensen, A. Baurichter, V. V. Petrunin, A. C. Luntz, The Journal of Chemical Physics 2002, 117, 5018-5030. 
[166] J. T. Kindt, J. C. Tully, The Journal of Chemical Physics 1999, 111, 11060-11069.

[167] G. Wahnström, Chemical Physics Letters 1989, 163, 401 - 406.

[168] V. Krishna, J. C. Tully, The Journal of Chemical Physics 2006, 125, 054706.

[169] J. C. Tully, M. Gomez, M. Head-Gordon, Journal of Vacuum Science E9 Technology A 1993, 11, 1914-1920.

[170] C. Springer, M. Head-Gordon, J. C. Tully, Surface Science 1994, 320, L57 - L62.

[171] J. T. Kindt, J. C. Tully, M. Head-Gordon, M. A. Gomez, The Journal of Chemical Physics 1998, 109, 3629-3636.

[172] A. C. Luntz, M. Persson, The Journal of Chemical Physics 2005, 123, 074704.

[173] A. Liebsch, Phys. Rev. B 1997, 55, 13263-13274.

[174] J. Trail, M. Graham, D. Bird, Computer Physics Communications 2001, 137, 163 - 173.

[175] P. Atkins, J. dePaula, Atkins' Physical Chemistry 8th ed., Oxford University Press, 2006.

[176] W. Unertl in Physical Structure, Vol. 1 of Handbook of Surface Science, W. Unertl (Ed.), North-Holland, 1996, pp. 3 - 49.

[177] G. Sachs, J. Weets, Zeitschrift für Physikalische Chemie 1930, 60, 481-490.

[178] M. Gajdoš, A. Eichler, J. Hafner, Journal of Physics: Condensed Matter 2004, 16, 11411164 .

[179] . Crljen, D. Šokčević, R. Brako, P. Lazić, Vacuum 2003, 71, 101 - 106, Proceedings of the 9th Joint Vacuum Conference (JVC-9), organised by the Austrian Vacuum Society, in cooperation with the Vacuum societies of Hungary, Croatia, Slovenia, Czech Republic, Slovakia and Italy, Schloss Seggau, Austria.

[180] Y.-D. Liu, V. Ozolins, The Journal of Physical Chemistry C 2012, 116, 4738-4747.

[181] V. Repain, J. M. Berroir, S. Rousset, J. Lecoeur, Europhysics Letters 1999, 47, 435-441.

[182] K. G. Huang, D. Gibbs, D. M. Zehner, A. R. Sandy, S. G. J. Mochrie, Phys. Rev. Lett. 1990, 65, 3313-3316.

[183] A. Hoss, U. Romahn, M. Nold, P. v. Blanckenhagen, O. Meyer, Europhysics Letters 1992, 20, 125-130.

[184] S. Narasimhan, D. Vanderbilt, Phys. Rev. Lett. 1992, 69, 1564-1567. 
[185] N. Takeuchi, C. T. Chan, K. M. Ho, Phys. Rev. B 1991, 43, 13899-13906.

[186] M. Mansfield, R. J. Needs, Journal of Physics: Condensed Matter 1990, 2, 2361.

[187] F. Ercolessi, M. Parrinello, E. Tosatti, Philosophical Magazine A 1988, 58, 213-226.

[188] F. Ercolessi, A. Bartolini, M. Garofalo, M. Parrinello, E. Tosatti, Surface Science 1987, 189-190, 636 - 640, Proceedings of the Ninth European Conference on Surface Science.

[189] A. P. Seitsonen, Surface Science 2016, 643, 150 - 155, Present challenges in surface science, a special issue in honour of Dietrich Menzel.

[190] R. Gaspari, C. A. Pignedoli, R. Fasel, M. Treier, D. Passerone, Phys. Rev. B 2010, 82, 041408.

[191] H. Bulou, C. Goyhenex, Phys. Rev. B 2002, 65, 045407.

[192] L. Stobiński, R. Duś, Vacuum 1994, 45, 299 - 301, Special Issue Proceedings of the 16th International Seminar on Surface Physics.

[193] L. Stobiński, R. Duś, Surface Science 1992, 269-270, 383 - 388.

[194] E. Lisowski, L. Stobiński, R. Duś, Surface Science Letters 1987, 188, L735 - L741.

[195] A. G. Sault, R. J. Madix, C. T. Campbell, Surface Science 1986, 169, 347 - 356.

[196] B. Schindler, D. Diesing, E. Hasselbrink, The Journal of Physical Chemistry C 2013, 117, $6337-6345$.

[197] B. Gergen, H. Nienhaus, W. H. Weinberg, E. W. McFarland, Science 2001, 294, 25212523 .

[198] D. Krix, R. Nünthel, H. Nienhaus, Phys. Rev. B 2007, 75, 073410.

[199] D. Krix, R. Nünthel, H. Nienhaus, Journal of Vacuum Science 83 Technology A 2007, 25, $1156-1160$.

[200] H. Nienhaus, H. S. Bergh, B. Gergen, A. Majumdar, W. H. Weinberg, E. W. McFarland, Phys. Rev. Lett. 1999, 82, 446-449.

[201] J. R. Trail, M. C. Graham, D. M. Bird, M. Persson, S. Holloway, Phys. Rev. Lett. 2002, 88,166802 .

[202] D. A. Kovacs, T. Babkina, T. Gans, U. Czarnetzki, D. Diesing, Journal of Physics D: Applied Physics 2006, 39, 5224. 
[203] C. Lamont, B. Persson, G. Williams, Chemical Physics Letters 1995, 243, 429 - 434.

[204] W. Eichenauer, D. Liebsch, Zeitschrift für Naturforschung A 1962, 17, 355.

[205] J. K. Nørskov, Physical Review B 1979, 20, 446-454.

[206] J. Nørskov, F. Besenbacher, Journal of the Less Common Metals 1987, 130, 475 - 490, Proceedings of the International Symposium on the Properties and Applications of Metal Vydrides V.

[207] P. Ferrin, S. Kandoi, A. U. Nilekar, M. Mavrikakis, Surface Science 2012, 606, 679-689.

[208] S. M. Janke, Master's thesis, Georg-August University Göttingen, Germany, 2012.

[209] K. Nobuhara, H. Nakanishi, H. Kasai, J. Appl. Phys. 2000, 88, 6897-6901.

[210] K. Nobuhara, H. Kasai, H. Nakanishi, W. A. Diño, Journal of Applied Physics 2004, 96, $5020-5025$.

[211] J. Nie, H. Xiao, X. Zu, CHEMICAL PHYSICS 2006, 321, 48-54.

[212] X.-Y. Pang, L.-Q. Xue, G.-C. Wang, Langmuir 2007, 23, 4910-4917.

[213] K. Gundersen, B. Hammer, K. W. Jacobsen, J. K. Nørskov, J. Lin, V. Milman, Surface Science 1993, 285, 27-30.

[214] A. Forni, G. Wiesenekker, E. J. Baerends, G. F. Tantardini, Journal of Physics: Condensed Matter 1995, 7, 7195.

[215] G. Lee, E. Plummer, Surface Science 2002, 498, 229 - 236.

[216] E. M. Mccash, S. F. Parker, J. Pritchard, M. A. Chesters, Surface Science 1989, 215, 363 $-377$.

[217] M. Luo, D. MacLaren, I. Shuttleworth, W. Allison, Chemical Physics Letters 2003, 381, $654-659$.

[218] M. Luo, D. MacLaren, W. Allison, Surface Science 2005, 586, 109 - 114.

[219] K. Mudiyanselage, Y. Yang, F. M. Hoffmann, O. J. Furlong, J. Hrbek, M. G. White, P. Liu, D. J. Stacchiola, The Journal of Chemical Physics 2013, 139, 044712.

[220] M. Luo, G. Hu, Surface Science 2009, 603, 1081 - 1086.

[221] D. V. Shalashilin, B. Jackson, The Journal of Chemical Physics 1998, 109, 2856-2864. 
[222] M. Bonfanti, B. Jackson, K. H. Hughes, I. Burghardt, R. Martinazzo, The Journal of Chemical Physics 2015, 143, 124704.

[223] G. Kresse, J. Furthmüller, Physical Review B 1996, 54, 11169-11186.

[224] G. Kresse, J. Furthmüller, Computational Materials Science 1996, 6, 15-50.

[225] G. Kresse, J. Hafner, Physical Review B 1993, 47, 558.

[226] G. Kresse, J. Hafner, Physical Review B 1994, 49, 14251.

[227] K. Levenberg, Quart. Appl. Math. 1944, 2, 164-168.

[228] D. W. Marquardt, Journal of the Society for Industrial and Applied Mathematics 1963, $11,431-441$.

[229] A. Kant, K. A. Moon, High Temperature Science 1979, 11, 55-62.

[230] J. Hafner, Journal of Computational Chemistry 2008, 29, 2044-2078.

[231] L. Böselt, Bachelor’ thesis, Georg-August University Göttingen, Germany, 2015.

[232] M. Kammler, Master's thesis, Georg-August University Göttingen, Germany, 2015.

[233] M. Kammler, 2016, Private Communications.

[234] B. Golding, S. C. Moss, B. L. Averbach, Physical Review 1967, 158, 637-646.

[235] H. Preston-Thomas, Metrologia 1990, 27, 3-10.

[236] C. Wöll, S. Chiang, R. J. Wilson, P. H. Lippel, Phys. Rev. B 1989, 39, 7988-7991.

[237] I. Wolfram research, Mathematica, 2015.

[238] G. Kresse, M. Marsman, J. Furthmüller, VASP the GUIDE, University of Vienna, 2012.

[239] D. R. Hamann, M. Schlüter, C. Chiang, Phys. Rev. Lett. 1979, 43, 1494-1497.

[240] D. Vanderbilt, Phys. Rev. B 1990, 41, 7892-7895.

[241] P. E. Blöchl, Phys. Rev. B 1994, 50, 17953-17979.

[242] G. Kresse, D. Joubert, Phys. Rev. B 1999, 59, 1758-1775.

[243] G. Kresse, M. Marsman, J. Furthmüller, VASP the GUIDE, http://cms.mpi.univie.ac.at/vasp/vasp/vasp.html, 2015.

[244] W. Humphrey, A. Dalke, K. Schulten, Journal of Molecular Graphics 1996, 14, 33-38.

[245] K. Refson, Physica B+C 1985, 131, $256-266$. 



\section{A. Appendix}

\section{A.1. VASP}

The Vienna ab initio simulation package (VASP) [223-226] is a simulation environment for electronic structure calculations. It allows quantum mechanical calculations on the level of Hartree Fock, DFT and Hybrid functionals including ab initio MD simulations. VASP uses a plane-wave basis set [238] and allows a choice between pseudopotential methods (e.g. Ref. [239, 240]) and projector augmented wave [241, 242] methods for the treatment of the core electrons during the calculations: pseudopotentials avoid treating the chemically inert core electrons by replacing them with nodeless potentials that regenerate the effect of the core electrons on the valence electrons [230]. VASP supplies ultra-soft pseudopotentials [240] that need less plane waves to construct, reducing the calculational effort. As ultra-soft pseudopotentials were used to calculate the AIMD-trajectories used for the fit, I naturally also assumed them to calculate the 3D grid for the H-atom. However, the VASP support now discourages the use of pseudopotentials and recommends to use projector augmented waves which are regarded to be generally more accurate than the ultra-soft pseudopotentials [238]. The PAW method [241, 242] is based on a frozen core approximation but reconstructs the exact valence wave function including all nodal features [230, 238].

VASP uses iterative matrix diagonalisation techniques to determine the electronic groundstates [238, 243]. I performed the VASP calculations on our own cluster. We have modified the VASP-code such that the SRP48-functional suggested by Díaz et al. [12, 121] can be used.

For DFT-calculations, I used the SRP48-, RPBE-, PBE- and PW91-GGA-functional with US-PPs. I used VASP to calculate the electronic density within the Au(111) slab with the SRPfunctional. If not stated otherwise, the information describing the VASP-calculations are taken from the VASP-manual [238] or the online manual [243].

\section{A.1.1. Input files}

The four mandatory files to start VASP calculations are the INCAR, POSCAR, POTCAR and KPOINTS-files. 
The INCAR-file is the control file for VASP calculations. Invoked by parameters, it contains information on all the options that are needed for the current calculations like, e.g. algorithms, exchange-correlation functionals and convergence as well as output options. The INCAR files I used for DFT-calculations are attached in the back of this chapter. The most important parameters are:

The GGA-parameter specifies which GGA-functional should be used to calculate the exchangecorrelation functional.

For metals, the step function used to evaluate the band-structure energy at $0 \mathrm{~K}$, converges very slowly with the number of k-points included, as the occupancies at the Fermi-level jump discontinuously from 1 to 0 . To circumvent this problem, partial occupancies are introduced and the step function is replaced by a smoother functions. ISMEAR offers the choice of this function. For ISMEAR $=-1$, the step function is replaced by a Fermi-Dirac function whose width is described by SIGMA $=k_{\mathrm{B}} T$. The introduction of the smearing leads to a generalized free energy that needs to be minimized instead of the total energy, but the total energy can be extrapolated with $\sigma \rightarrow 0$. Errors in the forces introduced by this scheme are expected to be small.

The PREC flag determines the precision of the VASP calculations by, if not otherwise specified, setting ENCUT and determining the size of the fast fourier transform grid for the calculations.

ENCUT is the energy cut-off for the pseudopotentials. It includes plane-waves up to a kinetic energy of $E_{\text {cut }}$ into the calculation and therefore determines the size of the basis set. It does not need to be specified, for there is already a default cut-off in the POTCAR-file (ENMAX).

The IBRION flag determines how the first and second derivatives of the energy with respect to the ionic positions are calculated. This allows to perform MD-simulations and the relaxation of the ionic positions as well as the determination of phonon frequencies and Hessian matrix.

LCHARG: This flag determines whether or not the charge densities are written out during the calculations.

ISPIN determines if the calculations are to be done spin-restrictedly or unrestrictedly. If the $\mathrm{H}$ atom is above the surface, then, the spin needs to be considered in the calculations.

The parameter that determines which iterative matrix diagonalisation technique should be used to calculate the electronic ground state is ALGO. The best choices are the residual minimization method with direct inversion of iterative subspace (RMM-DIIS), the blocked Davidson algorithm or a mixture of both. 


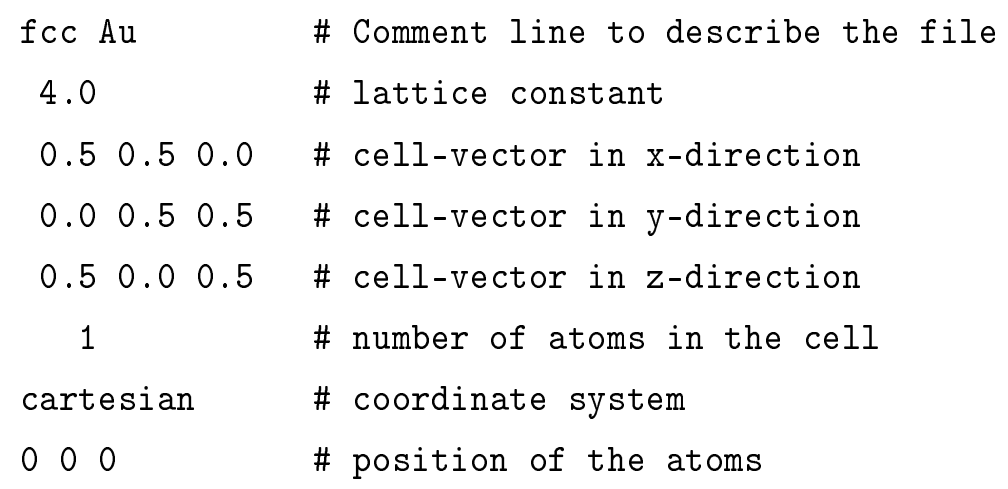

Figure A.1.1.: The POSCAR file. Example for a $1 \times 1 \times 1$ cell for a bulk-calculation.

\section{POSCAR}

The POSCAR-file contains the cell-geometry, the number of atoms and atomic species and their position for the problem under consideration. The first line (see Fig. A.1.1) is reserved for a comment to give information about the calculation the POSCAR file is used in. The second line contains the lattice constant and the next three lines contain the cell matrix that contains the cell vectors in $x$ - (line 3), $y$ - (line 4 ) and $z$-direction (line 5). The lattice constant is always multiplied onto the cell matrix, so for cells in which the lattice constant is just a factor, it should be written in the second line. Due to the periodic boundary conditions VASP employs when one moves from calculating bulk-properties to surface properties one needs to separate the periodic images from one another in one direction to create a surface, usually done in $z$-direction. The amount of space by which the slabs are separated is called the vacuum distance.

Line 6 contains the number of atoms in the cell. If there is more than one species in the cell, the first number denotes the number of atoms of the atomic species that comes first in the POTCAR-file, followed by the number of atoms of the second species and so on. The following line (line 7) describes in which coordinate system the atomic positions will be given. The choice here is between Cartesian coordinates and direct coordinates. Direct coordinates give the atomic positions between 0.0 and 1.0 and can be obtained by multiplying the Cartesian coordinates with the inverse of the cell matrix. From line 8 on, the positions of all atoms are given. Here, the atomic species must follow in the same order as specified in line 6 . This means if one writes a POSCAR-file containing one Au atom and two $\mathrm{H}$ atoms and writes in line 6 ' 12 ', then one has to write the position of the Au atom first, followed by the positions of the two hydrogen atoms.

The POSCAR-file also determines the cell dimensions for a cell containing a surface. They are denoted by $n_{x} \times n_{y} \times n_{z}$ where $n_{x}$ is the number of atoms in $x$-direction and $n_{y}$ and $n_{z}$ the number of atoms in $y$ - and $z$-direction, respectively. So, in the example in Fig.A.1.1, the cell dimensions are those of a $1 \times 1 \times 1$ cell and a $2 \times 2 \times 4$ cell would have four atoms in at its surface and four atoms in $z$-direction, that is four layers. 


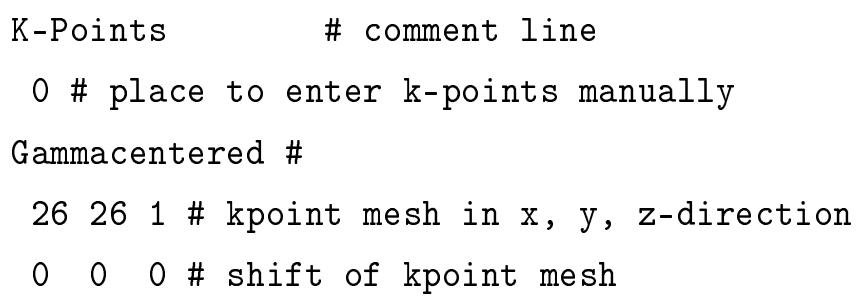

Figure A.1.2.: The KPOINTS file. Example for a simulation with a surface.

\section{POTCAR}

The POTCAR-file contains the pseudopotentials for all the atomic species in the calculation. It also provides information about the atoms, like their masses, their valences and specifics with regards to the way the pseudopotential were created. Furthermore, they contain a default energy cut-off radius which is chosen such that, in a bulk calculation, the error in the cohesive energy would be less than $10 \mathrm{meV}$. If more than one atomic species appears in the calculations, the two corresponding POTCAR-files need to be agglutinated. Care has to be taken here that the positions given for the individual atomic species in the POSCAR-file correspond to their ordering in the POTCAR-file.

\section{KPOINTS}

The KPOINTS-file gives the mesh in which the Brillouin-zone is sampled. The k-point mesh can be given either directly (line 2, Fig.A.1.2 by giving specific coordinates or automatically by entering a mesh size (line 4). For the automatic mesh generation, the method that should be used for the generation needs to be entered in the KPOINTS-file in line 3. The last line in the KPOINTS-file allows to shift the automatically generated mesh.

\section{A.1.2. Output Files}

OUTCAR This file is the main output file which contains, amongst others, the energy (and the energy $\sigma \rightarrow 0$ ) for a calculations, the stress tensor, basis-vectors and the forces. It also contains information on the ionic and electronic steps of the calculation and convergence information. What precisely is printed out can be determined using the NWRITE flag in the INCAR-file.

CHGCAR This file contains the lattice vectors and atomic coordinates but most importantly the total charge densities multiplied by the volume on a fine grid. It can be used to restart VASP. For visualization of the charge densities, VASP recommends the usage of the similar CHG-file.

\section{A.1.3. SRP input files}




\begin{tabular}{|c|c|c|}
\hline \multicolumn{3}{|c|}{$\mathrm{Au}(111) \quad 2 \times 2$ as in AIMD } \\
\hline \multicolumn{3}{|l|}{1.000000} \\
\hline 5.946767928 & .000000000 & .000000000 \\
\hline-2.973383964 & 5.150052372 & .000000000 \\
\hline .000000000 & .000000000 & 20.295320000 \\
\hline \multicolumn{3}{|l|}{161} \\
\hline \multicolumn{3}{|l|}{ Cartesian } \\
\hline .000000000 & .000000000 & .000000000 \\
\hline 2.973383964 & .000000000 & .000000000 \\
\hline-1.486691982 & 2.575026186 & .000000000 \\
\hline 1.486691982 & 2.575026186 & .000000000 \\
\hline 1.486692026 & .858342088 & -2.464000000 \\
\hline 4.46007 & .858342088 & -2.464000000 \\
\hline .000000044 & 3.433368273 & -2.464000000 \\
\hline 2.973384008 & 3.433368273 & -2.464000000 \\
\hline .000000000 & 1.716684175 & -4.878000000 \\
\hline 2.973383964 & 1.716684175 & -4.878000000 \\
\hline-1.486691982 & 4.291710361 & -4.878000000 \\
\hline 1.486691982 & 4.291710361 & -4.878000000 \\
\hline .000000000 & .000000000 & -7.335000000 \\
\hline 2.973383964 & .000000000 & -7.335000000 \\
\hline-1.486691982 & 2.575026186 & -7.335000000 \\
\hline 1.486691982 & 2.575026186 & -7.335000000 \\
\hline
\end{tabular}

Figure A.1.3.: An examplary POSCAR file for the 3D-grid using the SRP functional.

K-Points

0

Gammacentered

$2020 \quad 1$

000

Figure A.1.4.: The KPOINTS file for the 3D-grid using the SRP functional. 


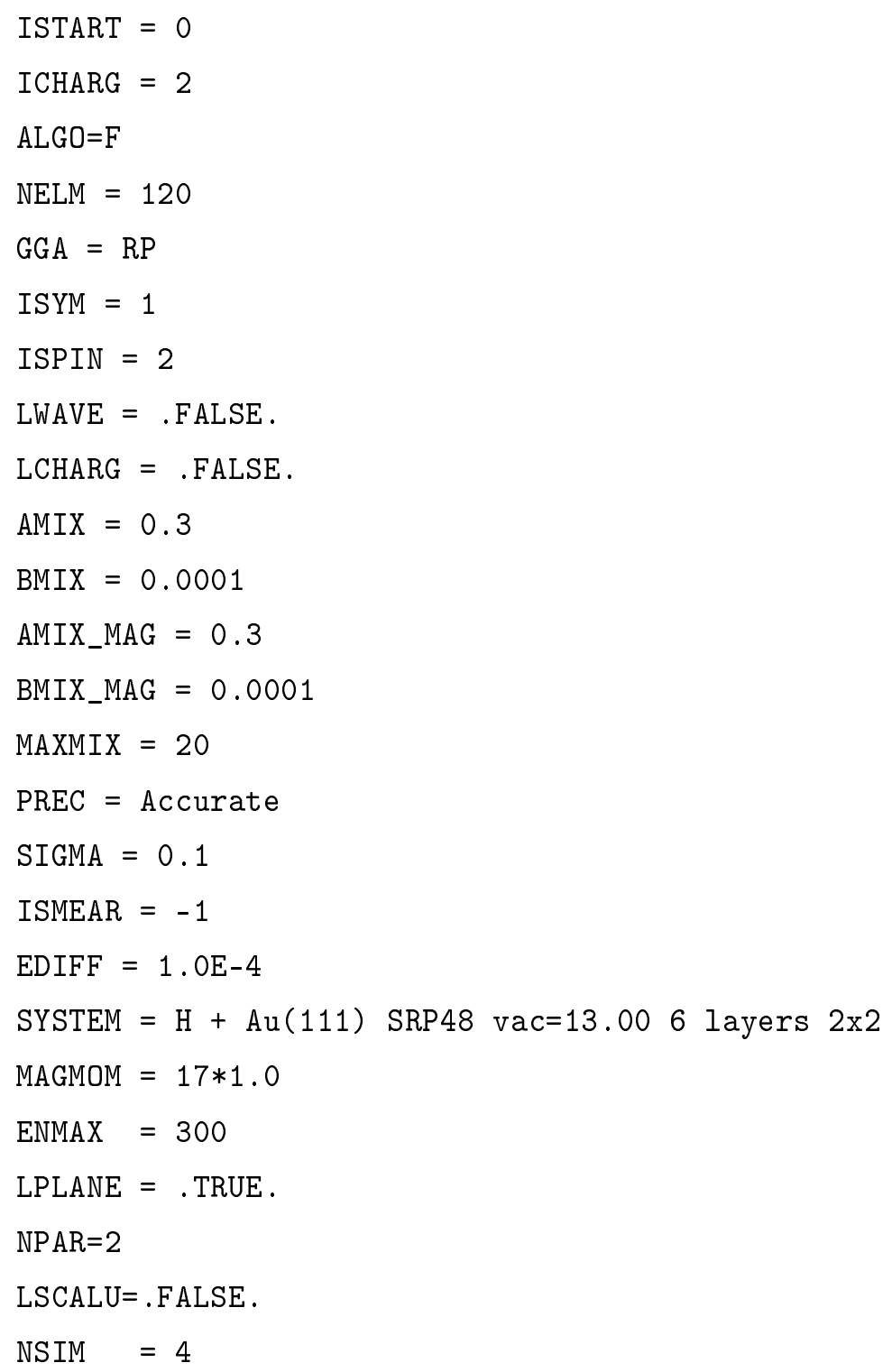

Figure A.1.5.: The INCAR file for the SRP 3D-grid calculations. 


\section{A.1.4. PBE input files}

PBE $\mathrm{aO}=4.181$

1.0

$\begin{array}{llll}5.91283 & 0.00000 & 0.00000\end{array}$

$\begin{array}{lll}-2.95641 & 5.12066 & 0.00000\end{array}$

$\begin{array}{lll}0.00000 & 0.00000 & 20.24170\end{array}$

161

Cartesian

$\begin{array}{lll}0.00000 & 0.00000 & 0.000000\end{array}$

$\begin{array}{lll}2.95642 & 0.00000 & 0.000000\end{array}$

$\begin{array}{lll}-1.4782 & 2.56033 & 0.000000\end{array}$

$\begin{array}{lll}1.4782 & 2.56033 & 0.000000\end{array}$

$\begin{array}{llll}4.43462 & 0.853444 & -2.42654\end{array}$

$\begin{array}{llll}0.00000 & 3.41377 & -2.42654\end{array}$

$2.95642 \quad 3.41377 \quad-2.42654$

$1.4782 \quad 0.853444-2.42654$

$\begin{array}{lll}0.00000 & 1.70689 & -4.81698\end{array}$

$2.95642 \quad 1.70689 \quad-4.81698$

$\begin{array}{lll}-1.4782 & 4.26721 & -4.81698\end{array}$

$1.4782 \quad 4.26721 \quad-4.81698$

$\begin{array}{llll}0.00000 & 0.00000 & -7.23088\end{array}$

$\begin{array}{llll}2.95642 & 0.00000 & -7.23088\end{array}$

$\begin{array}{lll}-1.4782 & 2.56033 & -7.23088\end{array}$

$1.4782 \quad 2.56033 \quad-7.23088$

$0.00000 \quad 0.00000 \quad 6.00000$

Figure A.1.6.: An examplary POSCAR file for the 3D-grid using the PBE functional.

K-Points

0

Gammacentered

881

$\begin{array}{lll}0 & 0 & 0\end{array}$

Figure A.1.7.: The KPOINTS file for the 3D-grid using the PBE functional. 


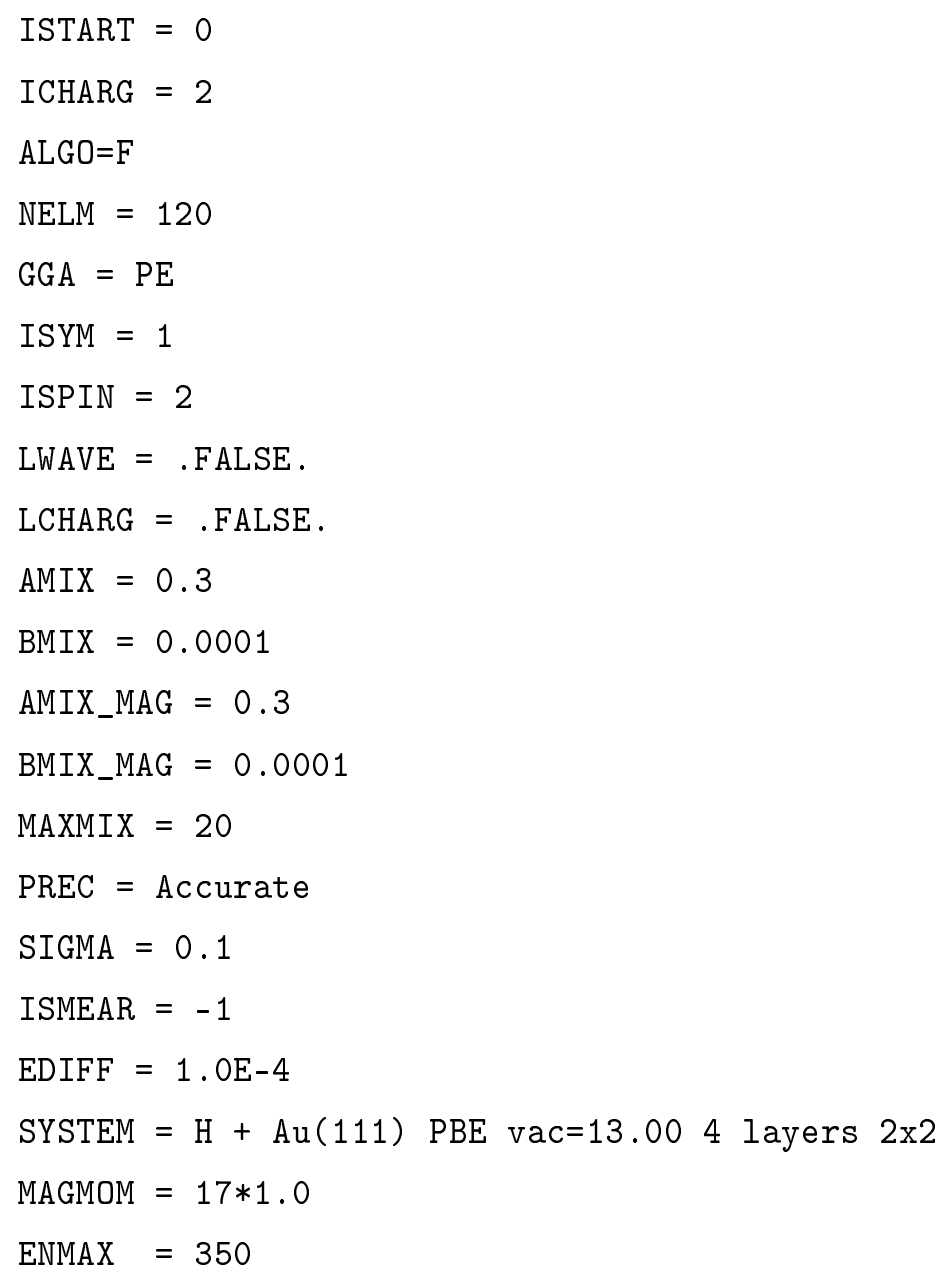

Figure A.1.8.: The INCAR file for the PBE 3D-grid calculations. 


\section{A.1.5. PW91 input files}

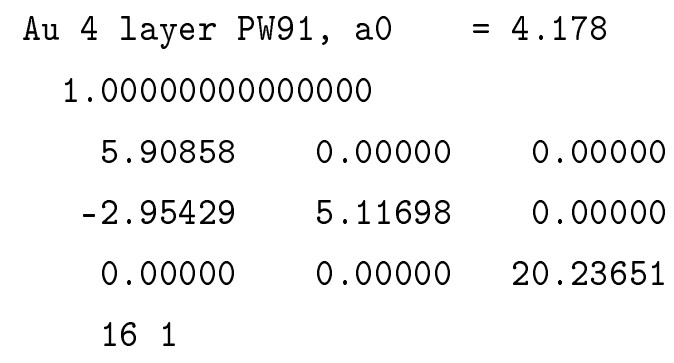

Cartesian

$\begin{array}{lll}0.00000 & 0.00000 & 0.00000\end{array}$

$\begin{array}{lll}2.95430 & 0.00000 & 0.00000\end{array}$

$\begin{array}{lll}-1.47714 & 2.55849 & 0.00000\end{array}$

$\begin{array}{lll}1.47714 & 2.55849 & 0.00000\end{array}$

$\begin{array}{llll}4.43144 & 0.85283 & -2.42854\end{array}$

$\begin{array}{llll}0.00000 & 3.41132 & -2.42854\end{array}$

$2.95430 \quad 3.41132 \quad-2.42584$

$\begin{array}{lll}1.47714 & 0.85283 & -2.42854\end{array}$

$\begin{array}{llll}0.00000 & 1.70566 & -4.81307\end{array}$

$2.95430 \quad 1.70566 \quad-4.81307$

$\begin{array}{lll}-1.47714 & 4.26415 & -4.81307\end{array}$

$\begin{array}{lll}1.47714 & 4.26415 & -4.81307\end{array}$

$\begin{array}{llll}0.00000 & 0.00000 & -7.22537\end{array}$

$\begin{array}{llll}2.95430 & 0.00000 & -7.22537\end{array}$

$\begin{array}{lll}-1.47714 & 2.55489 & -7.22537\end{array}$

$\begin{array}{lll}1.47714 & 2.55489 & -7.22537\end{array}$

$1.477146066-.8528306786 .00$

Figure A.1.9.: An examplary POSCAR file for the 3D-grid using the PW91 functional.

Gold

0

Gammacentered

11111

000

Figure A.1.10.: The KPOINTS file for the 3D-grid using the PW91 functional. 


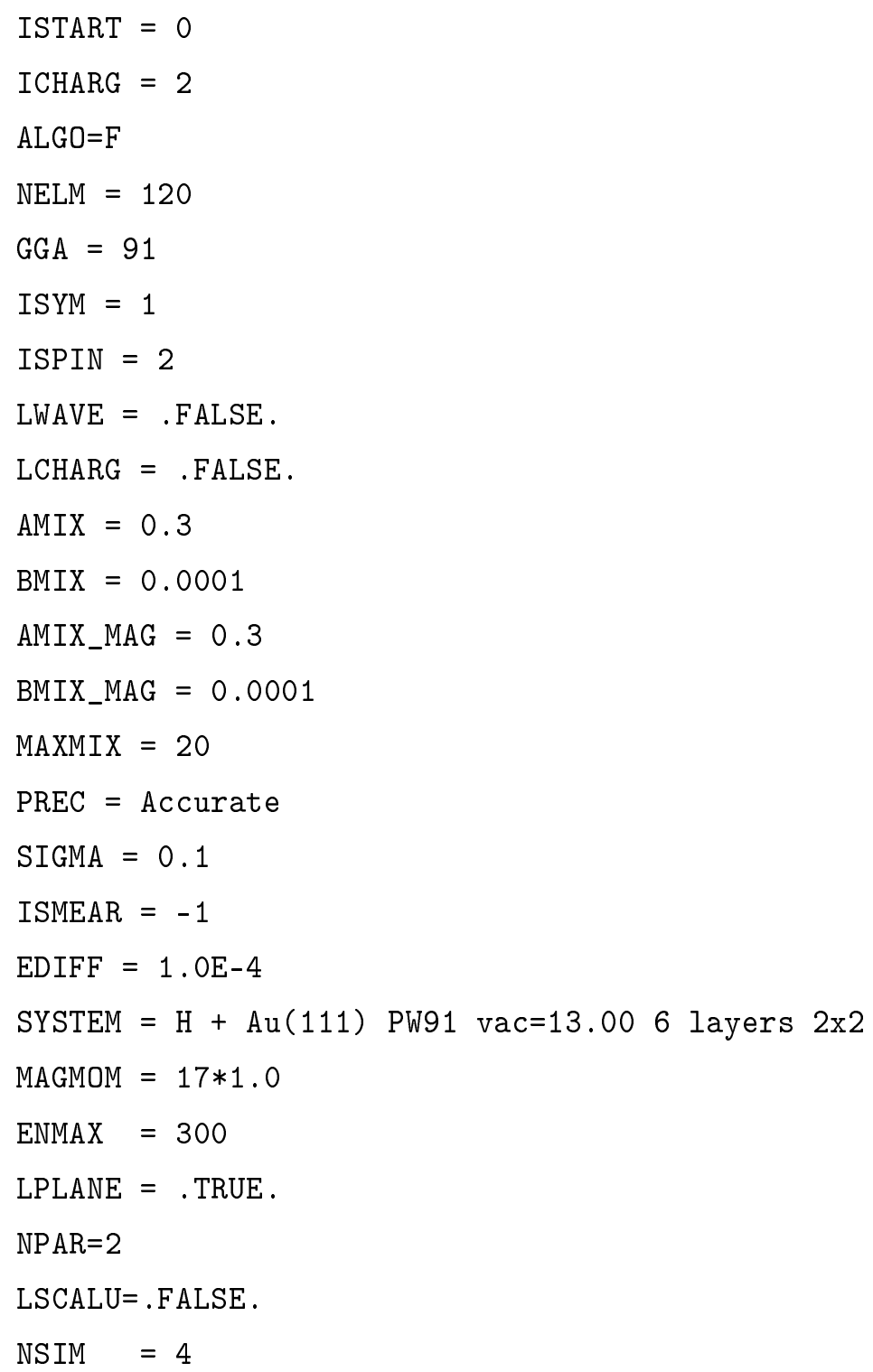

Figure A.1.11.: The INCAR file for the PW91 3D-grid calculations. 


\section{A.1.6. RPBE input files}

$$
\begin{array}{ccc}
\text { Au } 4 \text { layer RPBE, aO } & =4.225 \\
1.00000000000000 & \\
5.97505 & 0.00000 & 0.00000 \\
-2.98753 & 5.17455 & 0.00000 \\
0.00000 & 0.00000 & 20.34608 \\
161 & &
\end{array}
$$

$\begin{array}{rrr}5.97505 & 0.00000 & 0.00000 \\ -2.98753 & 5.17455 & 0.00000 \\ 0.00000 & 0.00000 & 20.34608\end{array}$

Cartesian
$\begin{array}{lll}0.00000 & 0.00000 & 0.00000\end{array}$
$\begin{array}{lll}2.98753 & 0.00000 & 0.00000\end{array}$
$\begin{array}{lll}-1.49376 & 2.58727 & 0.00000\end{array}$
$\begin{array}{lll}1.49376 & 2.58727 & 0.00000\end{array}$
$\begin{array}{llll}4.48129 & 0.862425 & -2.48508\end{array}$
$\begin{array}{llll}0.00000 & 3.44970 & -2.48508\end{array}$
$\begin{array}{llll}2.98753 & 3.44970 & -2.48508\end{array}$
$\begin{array}{llll}1.49376 & 0.862425 & -2.48508\end{array}$
$\begin{array}{llll}0.00000 & 1.72485 & -4.90678\end{array}$
$\begin{array}{llll}2.98753 & 1.72485 & -4.90678\end{array}$
$\begin{array}{lll}-1.49376 & 4.31212 & -4.90678\end{array}$
$\begin{array}{lll}1.49376 & 4.31212 & -4.90678\end{array}$
$\begin{array}{llll}0.00000 & 0.00000 & -7.34608\end{array}$
$\begin{array}{llll}2.98753 & 0.00000 & -7.34608\end{array}$
$\begin{array}{lll}-1.49376 & 2.58727 & -7.34608\end{array}$
$\begin{array}{llll}1.49376 & 2.58727 & -7.34608\end{array}$
0.00 .06 .00

Figure A.1.12.: An examplary POSCAR file for the 3D-grid using the RPBE functional.

K-Points

0

Gammacentered
881
$\begin{array}{lll}0 & 0 & 0\end{array}$

Figure A.1.13.: The KPOINTS file for the 3D-grid using the RPBE functional. 


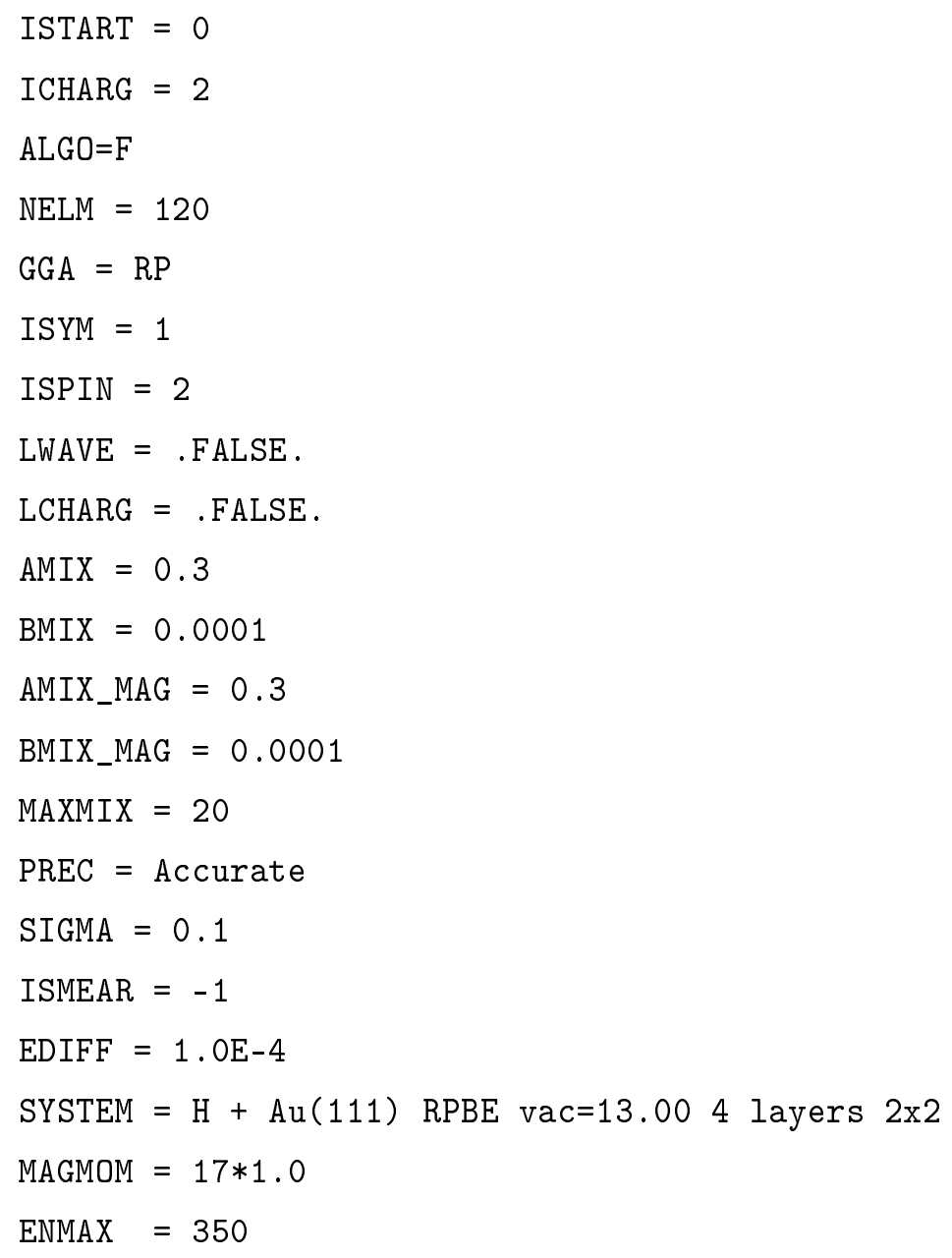

Figure A.1.14.: The INCAR file for the RPBE 3D-grid calculations. 


\section{B. MD_Tian Program}

MD_tian is a program for molecular dynamics calculations that Prof. Dr. Dan Auerbach, Dr. Alexander Kandratsenka and I wrote in FORTRAN to deal with our molecular dynamics simulations and fitting the EMT to DFT input data. The program's name derives from the Chinese expression 天下 (Tiānxià), meaning 'under one sky', thereby underscoring our aim to produce a program which includes all subprograms needed for our EMT-related purposes. '天' and '下' (symbolized by the underscore) are deliberately wrong way round in the program's name, because symbols on temples in China were commonly written from right to left. At the moment, the MDtianxia program calculates forces for the MD-simulations using the effective medium theory, but in future, the program shall be expanded to include also Lennard-Jones Potentials or other potential forms. The positions of the all species in the system are subjected to periodic boundary conditions.

The program takes three types of input files: a file that determines the geometry that should be used for the calculations, a file which includes the EMT parameters for each species used during the MD calculations and a control file. The control file, invoked by different flags, determines whether an MD-simulation or a fit is run and with which properties, the number of species and what kind of input data is used.

\section{B.1. The EMT-parameter file}

Apart for the suffix '.nml' the name of a parameter file can be chosen arbitrarily. Each chemical element has its separate parameter file. So far, we have chosen the following convention for naming the files: First, the potential to which the parameters refer, followed by the number of the fit from which the parameters stem and then, separated by an underscore, the element's name. So 'emt515_Au.nml' contains the parameters for the EMT potential for gold and stems from the fit No. 515. The file begins with a short comment line (see Fig.B.1.1. In the following lines, the name of the element is given followed by the list of the parameters. The order of lines in the file is fixed and must not be changed, otherwise the parameters will be wrongly assigned within the program. 


$\begin{array}{crl}\begin{array}{c}\text { Slab EMT Parameters } \\ \text { Name }=\text { Au }\end{array} & \text { \# Comment line } \\ \text { eta2 }= & \text { \# name of the species } \\ \mathrm{n} 0= & 3.29722801 & \text { \# List of parameters } \\ \mathrm{E} 0= & 0.04184152 & \\ \text { lambda }= & -3.80000000 & \\ \mathrm{~V} 0= & 4.18153000 & \\ \mathrm{kappa}= & 0.34752943 & \\ \mathrm{~s} 0= & 3.24943176 & \\ & 1.64174000 & \end{array}$

Figure B.1.1.: The parameter file for MD_tian.

\section{B.2. The Configuration files}

There are two types of configuration files the program can read in. Which type of file should be read in is specified with the conf-flag in the control file. The conf flag can be employed as shown in Tab. B.1. address signifies the absolute path to the file of the name 'file'; 'POSCAR' and 'fit' both need a POSCAR-file as input (see Sec.A.1.1). For the options 'geo' and 'conf', the program's own binary output mxt_conf $n$.bin-files (where $n$ can be any number between 99999999 and 00000000) are read in. They contain positions, velocities, accelerations and densities for all atoms. These files make it possible to restart an interrupted calculation, e.g. if one wants to start MD-simulations from an already equilibrated slab. Species that are already treated in the mxt_confn.bin-file need not be specified in the control file; another species may however be added. That means that if one has equilibrated a metal surface to a certain temperature, one can read in the information of this equilibration by means of an mxt_conf $n$.bin-file and add a particle to it by setting the particle-flag in the control file to start MD-simulations. In the latter case, the pip-flag needs to be specified in the control-file to give information on the initial positions of the particle. Likewise, the temperature only needs to be specified if one wants to continue simulations at a temperature that deviates from that of the mxt_conf $n$.bin-file.

If the option 'geo' is used, a specific mxt_confn.bin-file of the number $n$ (which configuration,

Table B.1.: Selection options for configuration files, to be inputted into the control file as shown. address signifies the absolute path to the file of the name 'file'.

\begin{tabular}{lllll}
\hline \hline conf & fit & 'address/file' & AIMD trajectory No. & fit number \\
conf & POSCAR & 'address/file' & \\
conf & conf & 'address/file' number of configurations & \\
conf & geo & 'address/file' & which configuration & \\
\hline \hline
\end{tabular}




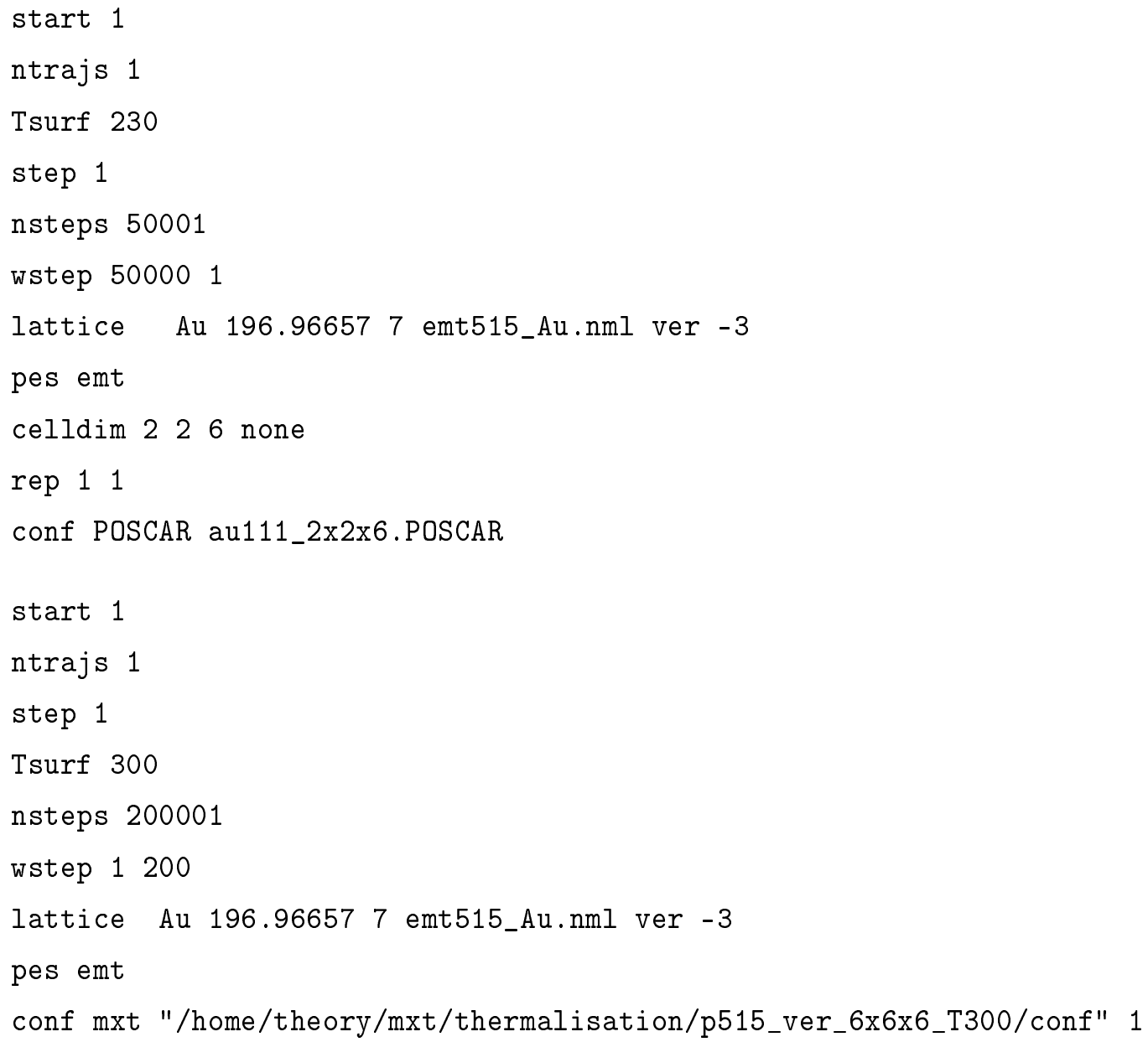

Figure B.3.1.: The control files used in MD_tian to create 1000 configurations of an equilibrated Au slab at $300 \mathrm{~K}$. Top: Control file to create a single file for an equilibrated configuration, bottom: control file to create 1000 further equilibrated configurations.

Tab.B.1 is read in. For 'conf', an mxt_confn.bin-file is chosen randomly from a batch of 'number of configurations' mxt_conf $n$.bin-files which makes it possible to start MD trajectory calculations from a number of different configurations for the equilibrated surfaces.

With the option 'fit', a fit can be run, which reads in the relaxed atom positions form a POSCAR-file and further needs the number of the AIMD-trajectory to which the fit is to be done, followed by the number of the fit.

\section{B.3. The Control File for MD simulations}

Upon execution, the mdtianxia program demands an input file which is the control file. It is usually named 'md_tian.inp' but its precise name has no influence on the program and it contains all the flags that are necessary to execute the program. Figure B.3.1 shows the control files used to equilibrate a slab and Fig. B.3.2 shows the control file that uses the configurations 
from the slab-equilibration as input to start an MD-simulation. The construction of equilibrated slab configurations that can be used as starting configurations works by first creating a single equilibrated configuration using the control file in Fig.B.3.1, top, and then, starting from that configuration, creating 1000 configurations in total which can be randomly sampled at the start of a trajectory (Fig. B.3.1, bottom). The flags are:

start is the number of the calculated trajectory.

ntrajs defines the number of trajectories that are to be calculated.

Tsurf gives the surface temperature that the slab should have.

step is the step in femtoseconds with which the trajectory is propagated.

nsteps are the number of steps made during the trajectory. So, nsteps.step gives the total length of the trajectory in fs.

wstep determines the type of the output file and when it is to be written. It will be explained in more detail in the following section (Sec.B.3.1).

lattice and projectile specifies the species of the slab and particle, respectively. At the moment, the EMT-implementation only offers two species in interaction with one another. Both flags are followed by the element abbreviation of the species, its atomic mass in atomic mass units, the number of the parameters, the name of the parameter file and the propagation algorithm $($ ver $=$ Verlet, lan $=$ Langevin, pef $=$ Verlet, but saving hypothetical energy loss to ehp during the calculation, bee=Beeman). For 'lattice', the last integer determines how many atoms are to be kept fixed to their positions during the propagation. A negative number denotes the number of layers, while a positive number affixes that number of atoms, starting from the first atom given in the configuration file for the slab. For 'projectile', the last integer determines the

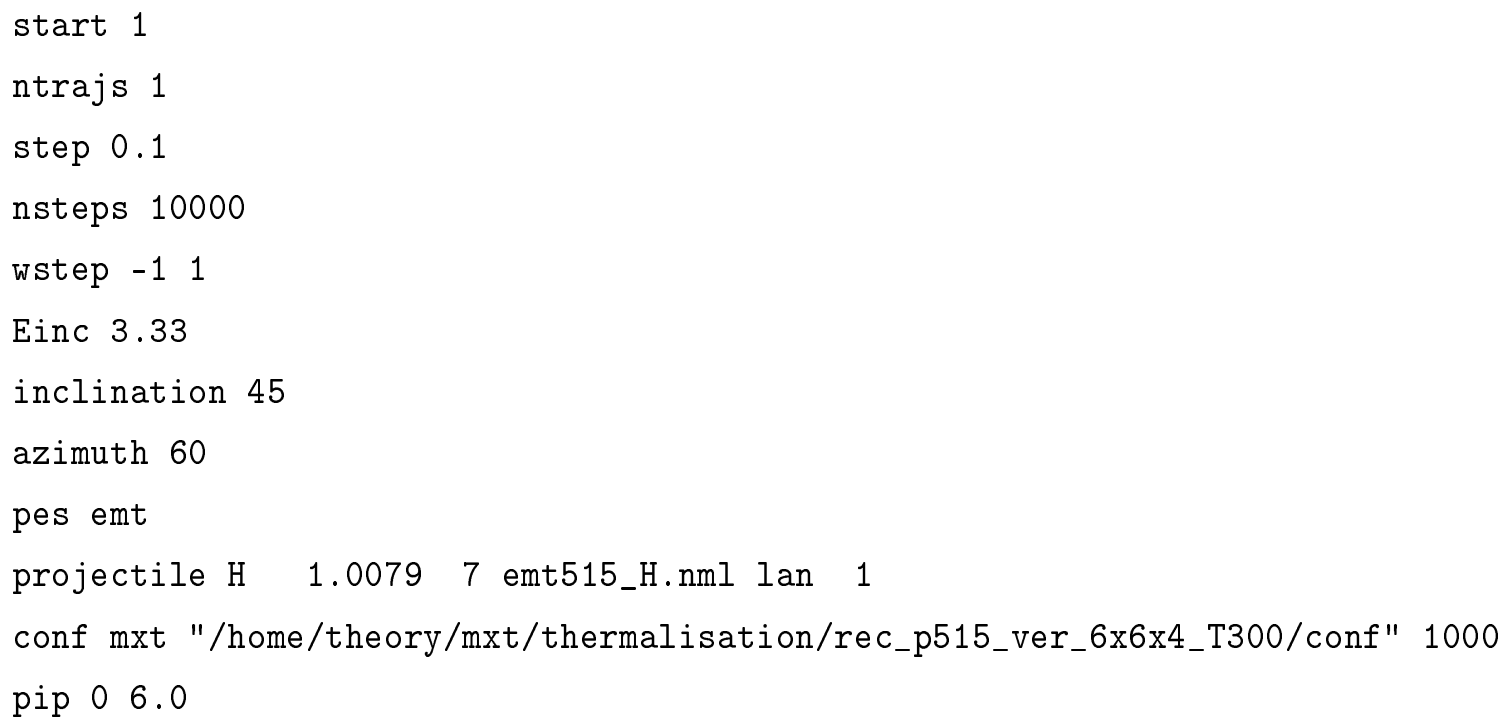

Figure B.3.2.: The control file used in MD_tian for a typical MD-simulation. 
number of particles in one periodic image.

pes This flag will in future offer the choice between different methods to calculate the forces and energies. At the moment, only EMT can be used.

celldim gives the dimensions of the slab. First, the number of atoms in $x$ - and $y$-direction is given and then the number of layers in $z$-direction. This option also allows to include ad-atoms or steps, for which the flag 'none' in line 10 of Fig.B.3.1, top, would have to be replaced by 'atlayer' followed by the number of atoms for each layer, starting from the surface.

rep gives the number of times the slab is to be repeated into $x$ - and $y$-direction. Although md_tian treats each problem within periodic boundary conditions, the EMT calculations need a supercell that includes up to the next-next-nearest neighbor atoms. This makes it necessary to increase the size of the input cell from usually $2 \times 2$ in $x y$-direction specified in the POSCAR file to $6 \times 6$. To put it in other words: this option allows the user to input a POSCAR-file of minimal extension in $x y$-direction $(2 \times 2)$, but use a much larger slab for the actual MD-simulation. A larger slab should circumvent the problem of artificial phonon-modes impressed upon the surface by small cells and periodic boundary conditions for longer time-scales.

conf offers the choice of the input file as described in the previous section (Sec.B.2).

Einc defines the incidence energy of the projectile in $\mathrm{eV}$.

inclination sets the polar incidence angle $\theta_{\text {in }}$ of the projectile. $\theta_{\text {in }}=0^{\circ}$ corresponds to normal incidence, $\theta_{\text {in }}=90^{\circ}$ to parallel incidence.

azimuth sets the azimuth incidence angle $\phi_{\text {in }}$ of the projectile. $\phi_{\text {in }}=0^{\circ}$ corresponds to scattering along the [1ํㅣㅇ-direction.

pip describes how the particle should start the calculation. pip $=0$ determines that only one particle is to be considered and that its position is to be chosen randomly. It is followed by the starting distance to the surface. The flag also allows to read in a specific starting position from the configuration file ( $p i p=-1)$, the specific impact site $(p i p=1)$, extra file to read in starting positions $(p i p=2)$ or starting positions determined by site-name ( $p i p=3)$.

\section{B.3.1. Output files}

The mdtianxia-program offers seven different output options which can be controlled with the flag wstep in the input file. The first slot for wstep determines which kind of output file is printed and the last, where it makes sense, in which step.

$\mathbf{w s t e p}(\mathbf{1})=\mathbf{m}$ saves all the information that is necessary to start the trajectory again into a binary file of the form mxt_conf $n$.bin (where $n$ can be any number between 99999999 and 00000000) after the $m^{\text {th }}$ step and then every $\operatorname{wstep}(2)^{\text {th }}$ step.

$\operatorname{wstep}(\mathbf{1})=\mathbf{0}$ produces files of the form mxt_trjn.dat. They contain the time step and the energies for species along the trajectory and the density, the position and the velocities of the projectile. If only 'lattice' is specified, it can be used to determine the temperature to which the 
slab equilibrates.

$\operatorname{wstep}(\mathbf{1})=-\mathbf{1}$ produces files of the form mxt_finn.dat which contain the initial conditions and energies and those at the end of the trajectory as well as the number of bounces and first five bounce sites.

$\operatorname{wstep}(\mathbf{1})=-\mathbf{2}$ produces files of the form mxt_conf $n$.xyz which can be read in with visualization programs and contain only the positions of the slab and particle atoms.

wstep(1) $=-\mathbf{3}$ produces a POSCAR-file at the end of the trajectory with the name mxt_annealn.POSCAR. It also contains information about the energies at the end of the trajectory.

wstep(1) $=-4$ produces files of the form mxt_rvn.dat which contain the initial conditions and energies and those at the saving-time of the trajectory. Here, the positions, velocities and densities are saved in every $\operatorname{wstep}(2)^{\text {th }}$ step of the trajectory.

$\operatorname{wstep}(\mathbf{1})=-\mathbf{5}$ produces files of the form mxt_conf $n$.pdb. These files have the pdb-format and can be used e.g. with the Visual Molecular Dynamics (VMD) package [244].

\section{B.4. The Propagation}

\section{B.4.1. Propagation Algorithms}

Propagation is done using either the Langevin or the Verlet algorithm. The program also offers the Refson-Beemann algorithm [245]. The velocity Verlet algorithm was implemented in the following form [60] where $\delta t$ is the time step, $\mathbf{r}$ is the position, $\mathbf{v}$ the velocity and $\mathbf{a}$ the acceleration.

$$
\begin{gathered}
\mathbf{v}\left(t+\frac{1}{2} \delta t\right)=\mathbf{v}(t)+\frac{1}{2} \delta t \mathbf{a}(t) \\
\mathbf{r}(t+\delta t)=\mathbf{r}(t)+\delta t \mathbf{v}\left(t+\frac{1}{2} \delta t\right) \\
\mathbf{v}(t+\delta t)=\mathbf{v}\left(t+\frac{1}{2} \delta t\right)+\frac{1}{2} \delta t \mathbf{a}(t+\delta t)
\end{gathered}
$$

For the Langevin-equation the extended Verlet algorithm was implemented in the following manner according to Allen and Tildesly [60]:

$$
\begin{gathered}
\mathbf{r}(t+\delta t)=\mathbf{r}(t)+c_{1} \delta t \mathbf{v}(t)+c_{2} \delta t^{2} \mathbf{a}(t)+\delta \mathbf{r}^{\mathrm{G}} \\
\mathbf{v}(t+\delta t)=c_{0} \mathbf{v}(t)+c_{1} \delta t \mathbf{a}(t)+\delta \mathbf{v}^{\mathrm{G}}
\end{gathered}
$$

For $\eta>0.01 \AA^{-3}$ and $T<0.0001 \mathrm{~K}$, the parameters can be calculated from the exact expression. Otherwise, the Taylor series was used.

$$
\begin{gathered}
c_{0}=\mathrm{e}^{-\eta \delta t} \approx 1-\eta \delta t+\frac{1}{2}(\eta \delta t)^{2} \\
c_{1}=(\eta \delta t)^{-1}\left(1-c_{0}\right) \approx 1-\frac{1}{2} \eta \delta t+\frac{1}{6}(\eta \delta t)^{2}
\end{gathered}
$$




$$
c_{2}=(\eta \delta t)^{-1}\left(1-c_{1}\right) \approx \frac{1}{2}-\frac{1}{6} \eta \delta t+\frac{1}{24}(\eta \delta t)^{2}
$$

and the stochastic integrals with $m$ being the mass of the particle subjected to the Langevin equations.

$$
\begin{gathered}
\delta \mathbf{r}^{G}=\int_{t}^{t+\delta t} \frac{d t^{\prime}}{m \eta}\left(1-\mathrm{e}^{-\eta\left(t+\delta t-t^{\prime}\right)}\right) \mathbf{F}^{\mathrm{st}}\left(t^{\prime}\right) \\
\delta \mathbf{v}^{G}=\int_{t}^{\delta t+t} d t^{\prime} \frac{\mathrm{e}^{-\eta\left(t+\delta t-t^{\prime}\right)}}{m} \mathbf{F}^{\mathrm{st}}\left(t^{\prime}\right)
\end{gathered}
$$

The stochastic force $\mathbf{F}^{\text {st }}$ is sampled from a bivariate Gaussian distribution.

\section{B.4.2. Fitting Procedure}

The fitting procedure employed a Levenberg-Marquardt [227, 228] damped least squares procedure which minimizes the rms deviation of the energy values given by DFT and the EMT PES. Since I have used the fitting routine, the Levenberg-Marquardt damped least square procedure has been replaced with an Trusted Region Nonlinear Least Squares with Linear Bound Constrains procedure based on the Levenberg-Marquardt procedure from the Intel MKL libraries which allows fitting with constrains. Since I have used the fitting procedure, it has been improved using a genetic algorithm [232, 233] whose description, since not relevant for the present thesis, will be omitted. A control file for the fit using this routine can be seen in Fig.B.4.1, also to be used with MD_tian. The flags in the control file that have not been mentioned in Section B.2 here are:

conf the last two numbers determine which AIMD trajectory is to be used for the fit (in Fig. B.4.1 that would be trajectory No. 817) and the number of the fit (2000)

trajname this one determines the total number of AIMD trajectories that are available and is followed by the number of all these trajectories. This allows to calculate the rms-error to a flexible number of AIMD configurations.

fitmix determines the number of points to be taken from the 3D-grid for the fit (Fig.B.4.1, line $8,700)$ and from the selected AIMD trajectory $(200)$.

evasp This is the reference energy calculated with VASP. Usually, this is the energy of a particle $6 \AA$ above the surface used in the calculations.

3Dgrid gives options for the 3D-grid. The first two numbers determine the lowest and highest energy value of configurations allowed in the fit. The second number determines the number of sites that are to be used in the fit, followed by their identification number as given in section 2.7 .1

aimd The first two numbers determine again the lowest and highest energy value belonging to configurations that are to be used in the fit. The third number gives the minimal distance of the particle to a surface atom in $\AA$ and the last one excludes distances from the surface above this number. 


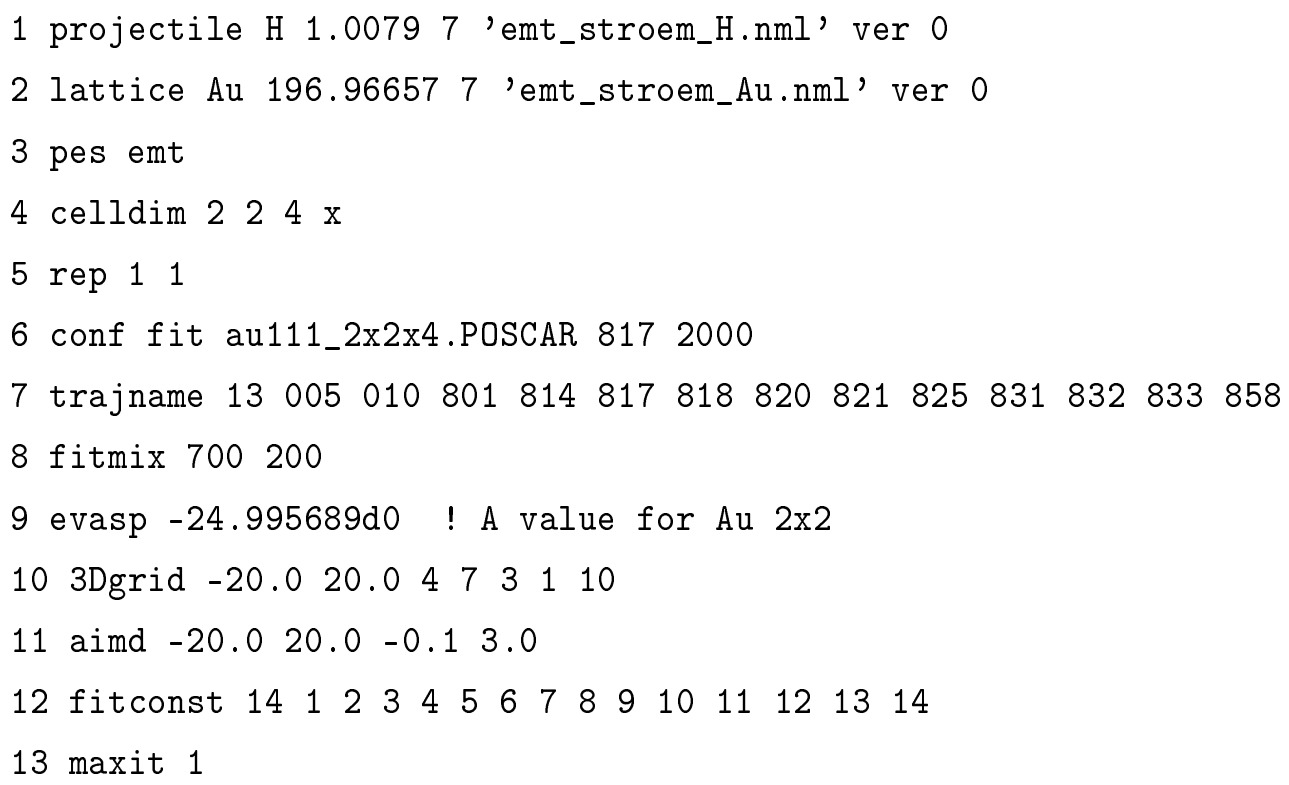

Figure B.4.1.: An exemplary control file for fitting with MD_tian.

fitconst determines which parameters are kept fixed to the values given in the parameter input files. The first number after the flag gives the number of parameters that are kept fixed (see Fig.B.4.1, line 12). The following numbers correspond to the numbers of the parameters. Parameters 1-7 are the parameters of the particle and 8-14 those of the slab according to the order of parameters in the EMT parameter file $\left(1,8=\eta, 2,9=n_{0}, 3,10=E_{0}, 4,11=\lambda, 5,12=V_{0}\right.$, $\left.6,13=\kappa, 7,14=s_{0}\right)$.

maxit is the maximal number of iterations.

Strictly speaking, a routine is implemented into the program that allows fitting the electron densities obtained from the VASP calculations. However, because the electron densities obtained from EMT and VASP are not the same entities, this routine has been commented out and is at present not serviceable.

\section{B.4.3. Surface Annealing}

To test and describe the stability of the reconstructed surface, I performed simulations with surface annealing with the MD_tian program. An exemplary control file I used to steer the simulated annealing is shown in Fig. B.4.2. The flags that were not described above are those that control the annealing procedure.

anneal controls the annealing procedure; the first number is the maximal temperature $T_{\max }$ that should be reached during annealing (in case of Fig. B.4.2 $700 \mathrm{~K}$, see line 11) and the second number is the number of steps $t_{\text {step }}$ for which a temperature should be simulated. The annealing starts at $\frac{2 t_{\text {step }}}{n s t e p} \cdot T_{\max }$, then, the surface is heated up in $n s t e p /\left(2 t_{\text {step }}\right)$ intervals to $T_{\max }$ and then 
goes down again to Tsurf. Each temperature interval is simulated for $t_{\text {step }}$ steps. The simulated annealing is repeated in ntrajs cycles.

To simulate annealing, the Langevin Dynamics are used as a thermostat. A higher friction coefficient of $\eta \approx 3 \cdot 10^{-3} \mathrm{fs}^{-1}$ was assumed. This makes the annealing simulations more effective and decreases the simulation time. I chose the friction coefficient for the simulated annealing to assume roughly the magnitude of friction an $\mathrm{H}$ atom would experience inside the gold surface. By probing higher and lower friction coefficient, I checked that the friction coefficient used for the simulated annealing gives the same results for structure and energy values after an annealing simulation.

\section{B.4.4. Influence of MD Simulation Conditions on Scattering Results}

To make sure that the simulation conditions I have chosen for the MD simulations are not cause of problems that could be prevented, I performed several tests whose results are presented in Tab.B.2 and Tab.B.3.

Problems that could be encountered are (1) a too small surface cell in $x$-, $y$ - direction that could lead to small oscillations imposed on the surface atoms by the periodic boundary conditions; I therefore tested if scattering results differ if I use a $10 \times 10$ surface cell instead of a $6 \times 6$ one and could observe no difference either in outcomes of the trajectory (Tab. B.2) or in energy loss behavior (Tab.B.3). (2) The number of layers could have an influence on the scattering results. I check for differences between using a 4- and a 6-layered slab and found it to be of little effect.

(3) If the simulations were curtailed too early, some $\mathrm{H}$ atoms might still retain enough energy after the end of the simulation to leave the surface, thereby changing the shape of the energy loss

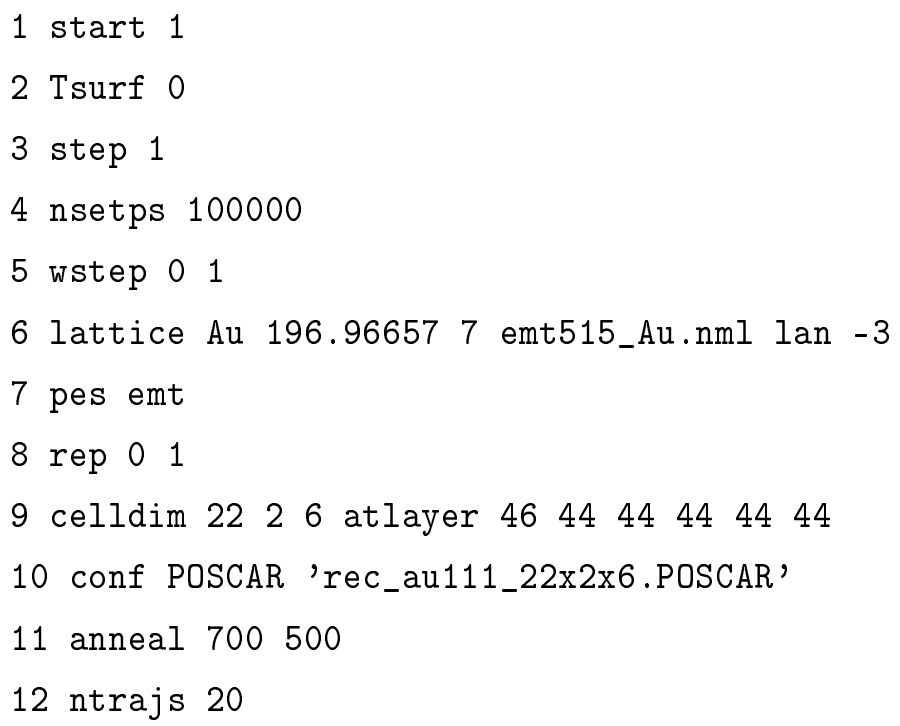

Figure B.4.2.: An exemplary control file for annealing simulations with MD_tian. 
Table B.2.: Outcomes (in \%) resulting from H atom collision with a $\mathrm{Au}(111)$ surface for nonadiabatic and adiabatic (in parenthesis) simulations. The incidence conditions are $E_{\text {inc }}=3.33 \mathrm{eV}$, $\theta_{\text {inc }}=45^{\circ}$ along the $[10 \overline{1}]$ surface direction.

\begin{tabular}{lllll}
\hline \hline Conditions & Scattering & $\begin{array}{l}\text { Surface } \\
\text { Adsorption }\end{array}$ & $\begin{array}{l}\text { Subsurface } \\
\text { Absorption }\end{array}$ & Transmission \\
\hline $6 \times 6 \times 6,10^{6}$ traj, $1 \mathrm{ps}$ & $55(82)$ & $23(4)$ & $21(6)$ & $1(8)$ \\
$6 \times 6 \times 6,10^{6}$ traj, $10 \mathrm{ps}$ & $55(82)$ & $25(6)$ & $19(5)$ & $1(8)$ \\
$6 \times 6 \times 4,10^{6}$ traj, $1 \mathrm{ps}$ & $55(80)$ & $23(3)$ & $18(4)$ & $4(14)$ \\
$10 \times 10 \times 4,10^{5}$ traj, $1 \mathrm{ps}$ & {$[55](80)$} & {$[23](3)$} & {$[18](4)$} & {$[4](14)$} \\
\hline
\end{tabular}

Table B.3.: Energy loss in \% of incidence energy for various outcomes resulting from $\mathrm{H}$ atom collision with a $\mathrm{Au}(111)$ surface for nonadiabatic and adiabatic simulations. The mean and maximum energy loss are shown for the total and differential ELD. The accuracy for specular scattering $\left(\theta_{\text {out }}=45^{\circ} \phi_{\text {out }}=60^{\circ}([10 \overline{1}])\right)$ has been reduced to account for the lower signal-to-noise ratio in the differential ELD. The incidence conditions are $E_{\mathrm{inc}}=3.33 \mathrm{eV}$, $\theta_{\text {inc }}=45^{\circ}$ along the $[10 \overline{1}]$ surface direction.

\begin{tabular}{lllll}
\hline \hline & Total & & \multicolumn{2}{l}{$\theta_{\text {out }}=45^{\circ} \phi_{\text {out }}=60^{\circ}$} \\
Conditions & Mean & Peak & Mean & Peak \\
\hline $6 \times 6 \times 6,10^{6}$ traj, $1 \mathrm{ps}$ & $35.3(4.95)$ & $14.0(1.65)$ & $33.5(2.25)$ & $14.9(1.35)$ \\
$6 \times 6 \times 6,10^{6}$ traj, $10 \mathrm{ps}$ & $35.3(4.95)$ & $14.0(1.65)$ & $33.2(2.25)$ & $14.9(1.35)$ \\
$6 \times 6 \times 64,10^{6}$ traj, $1 \mathrm{ps}$ & $35.3(4.65)$ & $14.9(1.65)$ & $31.7(2.25)$ & $15.2(1.05)$ \\
$10 \times 10 \times 4,10^{5}$ traj, 1 ps & $35.3(4.65)$ & $14.9(1.95)$ & $31.7(2.85)$ & $52.7(1.35)$ \\
\hline \hline
\end{tabular}

distributions and the scattering probabilities. I therefore checked that simulations propagated over 10 ps do not lead to markedly different results. Little differences are to be observed, but the changes are so slight that an increase in calculation time does not appear warranted unless long term diffusion is to be considered. The deviations in mean and peak energy loss for specular scattering for calculations with $10^{5}$ trajectories are due to the low signal-to-noise ratio.

The simulation conditions I chose are therefore very well suited to perform MD simulations.

\section{B.4.5. Disintegration Temperature}

I estimated the temperature at which the slab becomes unstable by equilibrating the slab at successively higher temperatures in steps of $50 \mathrm{~K}$ for 20 ps using a $6 \times 6 \times 6$ cell, and took the highest temperature at which the slab did not disintegrate as $T_{\text {stable. }}$. Whether or not the slab had disintegrated, I first at all check visually by looking at the structure of the slab throughout the trajectory and especially in the last step: if one of the atoms left its surface site, either 
by going into the gasphase or moving on top of the other surface atoms and thereby leaving a vacancy, I considered the slab as above its temperature of stability. I have not been able to link the melting temperature to other surface properties nor did I find a relation to the parameters of the fit. Since discerning the disintegration temperature visually is not very precise, I tested the disintegration temperature in large steps of $50 \mathrm{~K}$ and, if in doubt, always chose the lower temperature. This means that the disintegration temperatures $T_{\text {stable }}$ given in this thesis are a lower limit: the surface will definitely be stable up to this temperature, but it may also still be stable for $\approx 50 \mathrm{~K}$ above it. 



\section{Curriculum Vitae}

Name: $\quad$ Svenja Maria Janke

Address: $\quad$ Valentinsbreite 13

37077 Göttingen

Germany

Date of Birth: 20. November 1986

Place of Birth: Flensburg

Nationality: German

\section{Education}

1993 - 1997 Primary School: AFCENT International School, Brunssum, Netherlands

1997 - 1999 Grammar School: AFCENT International School, Brunssum, Netherlands

1999-2006 Grammar School: Abitur (A-level exams), Meldorfer Gelehrtenschule, Meldorf, Germany

2009 Bachelor of Science, Georg-August-University, Göttingen

Title: Nickel-Azid-Komplexe mit neuen, pyrazolatbasierten Liganden und deren magnetische Eigenschaften (Nickle-azide complexes with new, pyrazole-based ligands and their magnetic properties)

2009 Internship at the BASF-YPC Company Limited, Nanjing, People's Republic of China, Scholarship of the Dr. Helmut-Kraft Foundation

2012 Research stay of two weeks in the research group of Geert-Jan Kroes, Leiden, Netherlands

2012 Master of Science, Georg-August-University, Göttingen

Title: Development of a Potential Energy Surface for Hydrogen Atoms on a Gold(111) Surface 
2012 - present Ph.D. Project at the Georg-August-University of Göttingen

Title: Theoretical Description of Hydrogen Atom Scattering off Noble Metals

\section{List of Publications}

Oliver Bünermann, Hongyan Jiang, Yvonne Dorenkamp, Alexander Kandratsenka, Svenja M. Janke, Daniel J. Auerbach and Alec M. Wodtke, Electron-hole pair excitation determines the mechanism of hydrogen atom adsorption, Science 2015, 350, 1346 -1349.

S. M. Janke, Daniel J. Auerbach, Alec M. Wodtke and Alexander Kandratsenka, An Accurate full-dimensional potential energy surface for $\mathrm{H}-\mathrm{Au}(111)$ : Importance of nonadiabatic electronic excitation in energy transfer and adsorption, Journal of Chemical Physics 2015, $143(12), 124708$.

S. M. Janke, Michele Pavanello, Geert-Jan Kroes, Daniel Auerbach, Alec M. Wodtke and Alexander Kandratsenka, Toward Detection of Electron-Hole Pair Excitation in Hatom Collisions with $\mathrm{Au}(111)$ : Adiabatic Molecular Dynamics with a Semi-Empirical FullDimensional Potential Energy Surface, Z. Phys. Chem. 2013, 227, 1467 - 1490.

\section{Oral Presentations and Posters at Conferences and Workshops}

18.-22.03.2012 VIIIth Prague Workshop on Photoinduced Molecular Processes, Prague, Czech Republic

Oral Presentation: Constructing the Potential Energy Surface for Hydrogen Interacting with $\mathrm{Au}(111)$

09.-11.06.2012: Dynamics and Reactions of Hydrogen Atoms with Au and Ag-Surfaces: A Theoretical and Experimental Fundamental Research Cooperation (DRAGS), Banyuls-sur-Mer, France

Oral Presentation: A New Potential Energy Surface for $H$ on Au(111)

26.-30.11.2012: Dynamical Phenomena at Surfaces, Leiden, Netherlands

Poster: H at Au(111): A PES and Adiabatic MD Simulations

10.-15.03.2013: Winter School on Chemical Reaction Dynamics at Surfaces, Schloss Ringberg, Tegernsee, Germany

Poster: $H$ at Au(111): MD Simulations with a PES

24.-26.05.2013 DRAGS 2013, Hann. Münden, Germany

Oral Presentation: $H$ at Au(111): A PES and Adiabatic MD Simulations 
21.-26.07.2013 International Summer School on Basic Concepts and First Principles Computations for Surface Science: Applications in Chemical Energy Conversion and Storage, Norderney, Germany

Poster: H at Au(111): A DFT-based PES and Adiabatic MD Simulations

10-16.08.2013 Gordon Research Seminar and Conference 'Dynamics at Surfaces', Newport, RI, USA

Poster: H at Au(111): An Effective Medium Theory Full-dimensional PES

30.04.2014: Campus Seminar of the Max-Planck-Institute for Biophysical Chemistry

Oral Presentation: $H$ at Au(111): A Potential Energy Surface

18.-21.05.2014 Symposium on Dynamics at Surfaces, Schloss Ringberg, Tegernsee, Germany Oral Presentation: H at Au(111): A Potential Energy Surface

29.-31.10.2014 Dynamical Phenomena at Surfaces, Madrid, Spain

Poster: An Accurate and Full-Dimensional Potential Energy Surface for H/Au(111)

14.-16.05.2015 114th General Assembly of the German Bunsen Society for Physical Chemistry (Bunsentagung), Bochum, Germany

Poster: Constructing an Accurate and Full-dimensional Potential Energy Surface for $H / A u(111)$

20.-23.05.2015 Symposium on Dynamics at Surfaces, Schloss Ringberg, Tegernsee, Germany Oral Presentation: An Accurate Full-Dimensional Potential Energy Surface for $H$ at $A u(111)$

17.-19.06.2015 DRAGS 2015, Cahors, France

Oral Presentation: $H$ at Metal(111): Potential Energy Surfaces

08.-14.08.2015 Gordon Research Seminar and Conference 'Dynamics at Surfaces', Newport, RI, USA

Poster: H at Au(111) and Pd(111): Accurate Full-dimensional Potential Energy Surfaces

23.-28.08.2015 SUNCAT Summer Institute 2015 on Heterogeneous Catalysis for Energy and Society, Stanford, CA, USA

Poster: H at Au(111) and Pd(111): Accurate Full-dimensional Potential Energy Surfaces

01.-04.11.2015 International Bunsen Discussion Meeting: Nonadiabatic Effects in Chemical Kinetics, Dynamics, and Spectroscopy, Göttingen, Germany 
Oral Presentation: H Atom Scattering from Au(111): The Importance of Nonadiabatic Effects

10.12.2015 Extended evaluation of the MPI for Biophysical Chemistry 2015 by the Scientific Advisory Board

Poster: H at Au(111): Accurate Full-dimensional Potential Energy Surface

19.04.2016 Progress Reports, Institute for Physical Chemistry (IPC) Seminar, Göttingen, Germany

Talk: Molecular Dynamics Simulations on a Full-Dimensional Potential Energy Surface for $H$ scattering off Au(111) Poster: H at Au(111): Accurate Fulldimensional Potential Energy Surface

05.-07.05.2016 115th General Assembly of the German Bunsen Society for Physical Chemistry (Bunsentagung), Rostock, Germany

Poster: H at Au(111): Accurate Full-dimensional Potential Energy Surface

18-21.05.2016 Symposium on Dynamics at Surfaces, Schloss Ringberg, Tegernsee, Germany

Poster: H at Au(111): Accurate Full-dimensional Potential Energy Surface

\section{Achievements}

Poster Award: Bunsentagung 2015

Poster Award: Gordon Research Conference 'Dynamics at Surface', 2015 